\title{
APPLIED RESEARCH ON FIELD CROP DISEASE \& NEMATODE MANAGEMENT 2020
}

\author{
David B. Langston Jr., Extension Plant Pathologist \\ Hillary L. Mehl, Extension Plant Pathologist \\ Virginia Polytechnic Institute and State University \\ College of Agriculture and Life Sciences \\ Tidewater Agricultural Research \& Extension Center \\ Suffolk, Virginia 23437
}

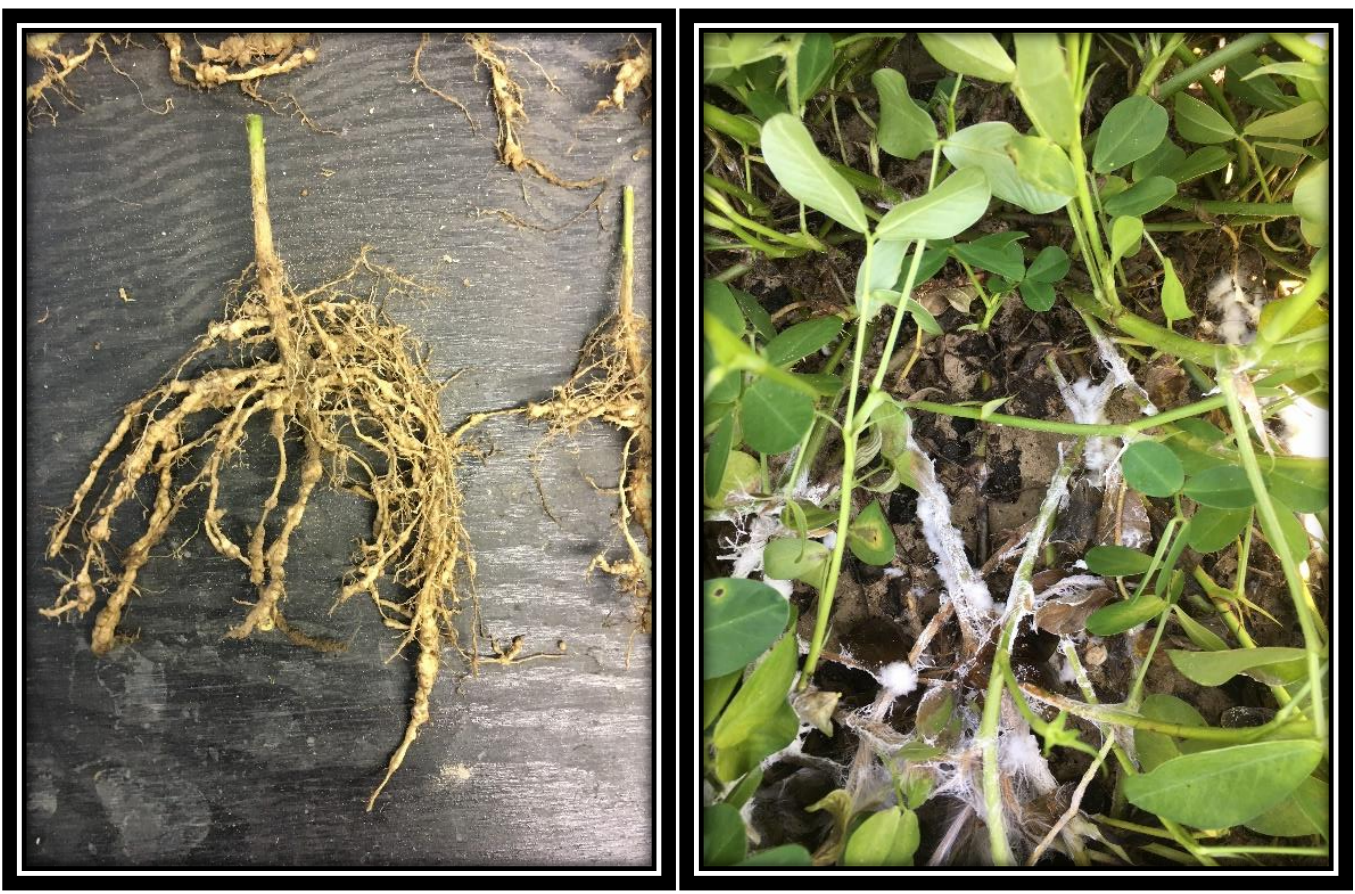

Virginia Cooperative Extension programs and employment are open to all, regardless of age, color, disability, gender, gender identity, gender expression, national origin, political affiliation, race, religion, sexual orientation, genetic information, veteran status, or any other basis protected by law. An equal opportunity/affirmative action employer. Issued in furtherance of Cooperative Extension work, Virginia Polytechnic Institute and State University, Virginia State University, and the U.S. Department of Agriculture cooperating. Edwin J. Jones, Director, Virginia Cooperative Extension, Virginia Tech, Blacksburg; M. Ray McKinnie, Administrator, 1890 Extension Program, Virginia State University, Petersburg. 


\section{ACKNOWLEDGMENTS}

The authors wish to thank the Virginia Agricultural Experiment Station and the many cooperators and contributors who provided the resources needed to support this applied research program. Special recognition is extended to Linda Byrd-Masters and Steve Byrum for technical skills in managing the Peanut/Cotton InfoNet, four online weather stations, field trials, data organization and processing, laboratory procedures, and various other duties in assisting with constructing this report. Also, to graduate students Joseph Opoku, Navjot Kaur, and Xing Wei who assisted with disease ratings in field trials. The general assistance of all AREC faculty and their technicians throughout the growing season was greatly appreciated. Thank you to Dr. Sally Taylor and Sean Malone who conducted thrips counts in our trials, to Dr. Hunter Frame and his department for assistance in cotton ginning, and to our farm and assistant farm managers Karl Jones and Brad Slye for general maintenance of field plots. We are grateful for the assistance of Patrick Robinson at Virginia Tech in updating programs for the Peanut/Cotton InfoNet as well as for Dr. Dave Walker who is recognized for running climatology forecast models and issuing frost advisories for peanut growers on the Peanut/Cotton InfoNet. Appreciation is expressed to Dr. Jon Eisenback who helped with this research by processing and identifying nematode populations in soil samples from cotton, soybean, and peanut trials. Collectively, the contributions of colleagues, professionals, students and growers were responsible for a highly successful and productive program to evaluate products and practices for disease management in field crops. 


\section{TABLE OF CONTENTS}

ACKNOWLEDGMENTS .

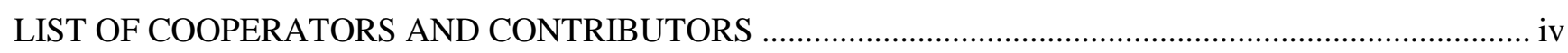

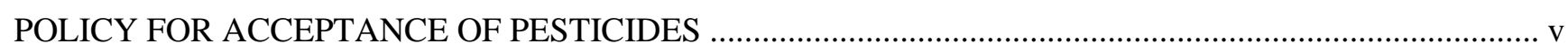

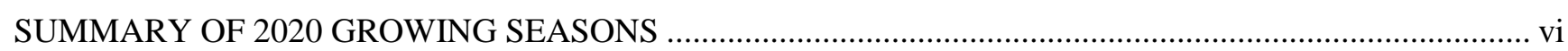

Evaluation of the combined effects of fungicide treatment and genetic resistance on FHB and

DON in wheat (WHTSCAB120, Tidewater AREC, Field 61B)

Evaluation of fungicide treatment and application timing for control of FHB and impact on

yield in wheat (WHTSCAB220, Tidewater Research Farm, Field 9B)

Comparison of fungicide treatments for foliar disease control and impact on yield in wheat

(WHTFOLFUN120, Tidewater AREC Duke Farm, Field 45B)

Comparison of fungicide treatments for foliar disease control and impact on yield in wheat

(WHTFOLFUN220, Tidewater Research Farm, Field 28)

Comparison of fungicide treatments for foliar disease control and impact on yield in wheat

(WHTFOLFUN320, Tidewater Research Farm, Field 28)

Evaluation of the combined effects of fungicide treatment and genetic resistance on FHB in

malt barley (BARSCAB120, Tidewater AREC, Field 61B)

Evaluation of fungicide treatment and application timing for FHB control in malt barley and impact on yield (BARSCAB220, Tidewater Research Farm, Field 9B)

Comparison of nematicide seed treatments and in-furrow nematicides for impact on plant health and yield in corn (CORNNEMA220, Tidewater AREC, Field 55)...

Evaluation of fungicides and spray timing for control of foliar disease in corn and impact on

yield at two locations, site 1 (CORNFOLFUN120, Tidewater AREC Duke Farm, Field 45A).

Evaluation of fungicides and spray timings for control of foliar disease in corn and impact

on yield at two locations, site 2 (CORNFOLFUN220, Tidewater AREC, Field 46B)

Evaluation of fungicide treatments for control of southern stem rot in peanut and impact

on yield (PTSSR120, Tidewater AREC, Field 46A).

Evaluation of fungicide treatments for control of Sclerotinia blight in peanut and impact on yield (PTSCL120,

Tidewater AREC, Field 46A)

Evaluation of fungicide treatments for control of peanut leaf spot and Sclerotinia blight

and impact on yield (PTLFSPOT120, Tidewater Research Farm, Field 34B).

Comparison of fungicide treatments for control of peanut leaf spot and impact on yield

(PTLFSPOT220, Tidewater Research Farm, Field 29) 
Evaluation of fungicide treatments for control of peanut leaf spot and impact on yield at three locations, site 1 (PTLFSPOT320, Tidewater Research Farm, Field 29)

Evaluation of fungicide treatments for control of peanut leaf spot and Sclerotinia blight and impact on yield at three locations, site 2, (PTLFSPOT420, Tidewater Research Farm, Field 34B)

Evaluation of fungicide treatments for control of peanut leaf spot and Sclerotinia blight and impact on yield at three locations, site 3, (PTLFSPOT520, Tidewater AREC, Field 46A). 71

Evaluation of fungicide seed treatments and seeding rate for control of damping-off diseases and impact on plant health and yield in cotton (COTSEEDFUN120, Tidewater Research Farm, Field 16B)

Evaluation of in-furrow and seed treatment nematicides for control of plant parasitic nematodes in cotton and impact on yield (COTSEEDNEMA120, Tidewater Research Farm, Field 16B) 83

Comparison of fungicide treatments for foliar disease control in soybean and impact on yield (SOYFOLFUN120, Tidewater AREC, Field 56)

Comparison of fungicide treatments for foliar disease control in soybean and impact on yield (SOYFOLFUN220, Tidewater AREC, Field 56)

Climatological Summary of the 2020 Growing Season at the Tidewater Agricultural Research \& Extension Center, Suffolk, VA..... 


\section{LIST OF COOPERATORS AND CONTRIBUTORS}

Virginia Polytechnic Institute and State University, and Virginia Agricultural Experiment Station Karl Jones, Farm Manager, Tidewater AREC

Brad Slye, Assistant Farm Manager, Tidewater AREC

Dr. Hunter Frame, Interim Director, Tidewater AREC

Dr. Maria Balota, Tidewater AREC

Dr. Sally Taylor, Tidewater AREC

Dr. David Holshouser, Tidewater AREC

Dr. Wade Thomason, School of Plant \& Environmental Sciences

Dr. Jon Eisenback, School of Plant \& Environmental Sciences

Patrick G. Robinson, CALS Information Technology

Growers and/or land owners

M. L. Everett, Capron, VA ................................Weather station

Glenn H. Hawkins, Skippers, VA ......................Weather station

Kevin Monahan, Waverly, VA..........................Weather station

County Extension Agents

Taylor Clarke, Mecklenburg County

Ursula Deitch, Northampton County

Roy Flanagan, Virginia Beach

Josh Holland, Southampton County

Watson Lawrence, Chesapeake

Laura Maxey-Nay, Hanover County

Mike Parrish, Dinwiddie County

Elizabeth Pittman, Suffolk

Theresa Pittman, Accomack County

Livvy Preisser, Isle of Wight County

Scott Reiter, Prince George County

Stephanie Romelczyk, Westmoreland County

Sara Rutherford, Greensville County

Commodity Groups and Organizations

Cotton Incorporated

Cotton Foundation, Seedling Disease and Nematode Control Committees

National Cottonseed Treatment Program

Virginia Cotton Board

Virginia Corn Board

Virginia Peanut Board

Virginia Soybean Board

Virginia Peanut Growers Association / National Peanut Board

Virginia Small Grains Board

US Wheat Barley Scab Initiative 
Private Companies

Albaugh, LLC, St. Joseph, MO

BASF Corporation, Raleigh, N.C.

Bayer CropScience, Kansas City, MO

Corteva AgriScience, Wilmington, DE

FMC Corporation, Philadelphia, PA

Leone BioVentures, Roanoke, VA

Nichino America, Wilmington, DE

Syngenta Crop Protection, Wilmington, DE

Valent U.S.A. LLC, Walnut Creek, CA

Spectrum Technologies, Aurora, IL

\section{POLICY FOR ACCEPTANCE OF PESTICIDES FOR TESTING}

Research on synthesis and exploration of agricultural chemicals and biotechnology for use in pest control continues to provide new materials for field evaluation. Compounds are being made available by private companies and universities for local research in a variety of ways; ranging from a sample with a code number to a thoroughly-tested material, with secure patents, technical data sheets, and comprehensive résumés of results of laboratory and field trials. Unfortunately, it is not possible for a scientist to include all materials and use patterns in a field research demonstration program. Therefore, materials are selected according to (i) overall need for a product in a particular crop or problem area, and (ii) overall promise of the material to improve crop management at the local level.

Before a material can be accepted for testing, the following descriptive information is required: (i) a list of the spectrum of biological activity, (ii) data on phytotoxicity and suggested rates of application, (iii) methods of application, (iv) available formulations, (v) mammalian toxicity $\left(\mathrm{LD}_{50}\right.$ ), (vi) possible health hazards, and (vii) potential hazards to the environment. Additional information that would be desirable includes: (i) identity of the active ingredient(s) and inert materials, (ii) physical properties (solubility, MP, VP, stability, etc.), (iii) residue information, (iv) residual soil life, (v) EPA residue tolerance (if any) and registration status, (vi) patent status, and (vii) unit cost in commercial markets.

Upon completion of field applications, it is the responsibility of the sponsor to dispose of all unused test materials. Because of limited space in controlled pesticide storage facilities and expenses associated with shipping and disposal, all sponsors are encouraged to ship not more than 1.5 times the anticipated quantity needed to complete a test.

The use of brand names and any mention or listing of commercial products or services in this publication does not imply endorsement by Virginia Tech nor discrimination against similar products or services not mentioned. Individuals who use agricultural chemicals are responsible for ensuring that the intended use complies with current regulations and conforms to the product label. Not all pesticides in this publication were applied according to their labels, and some of the tested chemicals are not yet registered for use in field crops in Virginia. Be sure to obtain information about usage regulations and examine a current product label before applying any chemical. For assistance, contact your county Extension agent. 


\section{SUMMARY OF 2020 GROWING SEASON}

Table 1. Comparison of rainfall, peanut heat units $\left(\mathrm{DD}_{56}\right)$ and cotton degree-days $\left(\mathrm{DD}_{60}\right)$ in 2020 to an average of historical records at the Tidewater AREC.

\begin{tabular}{|c|c|c|c|c|c|c|c|c|c|}
\hline \multirow[b]{2}{*}{ Month } & \multicolumn{9}{|c|}{ Rainfall (in.) } \\
\hline & 2013 & 2014 & 2015 & 2016 & 2017 & 2018 & 2019 & 2020 & Normal $^{1}$ \\
\hline May & 3.60 & 2.76 & 0.55 & 3.92 & 4.72 & 4.11 & 3.00 & 3.50 & 3.52 \\
\hline Jun & 8.10 & 3.95 & 7.48 & 5.82 & 2.78 & 4.05 & 5.55 & 3.80 & 4.31 \\
\hline Jul & 3.50 & 4.99 & 4.62 & 3.85 & 2.36 & 8.00 & 7.21 & 2.10 & 4.95 \\
\hline Aug & 6.40 & 2.14 & 2.62 & 2.19 & 7.33 & 6.10 & 7.52 & 8.50 & 5.16 \\
\hline Sep & 2.00 & 7.04 & 5.33 & 7.65 & 3.70 & 4.44 & 1.55 & 10.20 & 5.55 \\
\hline Oct & 4.50 & 2.30 & 3.56 & 5.11 & 2.32 & 3.50 & 3.98 & 1.30 & 3.65 \\
\hline Total & 28.10 & 23.18 & 24.16 & 28.54 & 23.21 & 30.23 & 28.81 & 29.40 & 27.13 \\
\hline
\end{tabular}

\begin{tabular}{cccccccccc} 
& \multicolumn{7}{c}{ Peanut Heat Units (DD56) } \\
\cline { 2 - 9 } Month & 2013 & 2014 & 2015 & 2016 & 2017 & 2018 & 2019 & $\mathbf{2 0 2 0}$ & Avg. $^{2}$ \\
\hline May & 355 & 437 & 463 & 324 & 386 & 520 & 531 & 301 & 384 \\
Jun & 580 & 598 & 686 & 577 & 681 & 625 & 581 & 572 & 583 \\
Jul & 707 & 659 & 724 & 766 & 790 & 654 & 731 & 797 & 696 \\
Aug & 589 & 609 & 635 & 735 & 628 & 687 & 639 & 698 & 642 \\
Sep & 390 & 513 & 522 & 550 & 461 & 459 & 530 & 436 & 455 \\
Oct & 255 & 266 & 230 & 277 & 309 & 230 & 298 & 255 & 228 \\
\hline Total & 2876 & 3082 & 3260 & 3229 & 3255 & 3390 & 3310 & 3059 & 2988 \\
\hline
\end{tabular}

Cotton Degree Days (DD 60

\begin{tabular}{cccccccccc} 
Month & 2013 & 2014 & 2015 & 2016 & 2017 & 2018 & 2019 & $\mathbf{2 0 2 0}$ & Avg. $^{3}$ \\
\hline May & 260 & 331 & 359 & 226 & 286 & 404 & 418 & 211 & 284 \\
Jun & 463 & 484 & 567 & 459 & 570 & 505 & 464 & 454 & 466 \\
Jul & 583 & 535 & 600 & 643 & 674 & 531 & 608 & 679 & 563 \\
Aug & 469 & 485 & 512 & 611 & 505 & 564 & 515 & 574 & 513 \\
Sep & 295 & 397 & 409 & 430 & 351 & 504 & 417 & 331 & 344 \\
Oct & 169 & 185 & 153 & 205 & 229 & 202 & 204 & 169 & 159 \\
\hline Total & 2239 & 2417 & 2600 & 2574 & 2615 & 2710 & 2626 & 2418 & 2328 \\
\hline
\end{tabular}

${ }^{3} \mathrm{Avg}$. is mean of previous 23 yrs (1995-2019). Data for 1995-2012 were according to records from a NOAA station (44-4044) located at Tidewater AREC, Holland Rd., Suffolk, VA; data for 2013 through present were recorded from a Spectrum Watchdog weather station located at the Tidewater Research Farm, Hare Rd., Suffolk, VA. Observation period for data includes 1 May through 31 Oct. 
TEST ID: WHTSCAB120

PURPOSE: To evaluate the combined effects of fungicide treatment and genetic resistance on FHB and DON in wheat

LOCATION: Tidewater AREC, 6321 Holland Rd., Suffolk, VA

CROP INFORMATION:

\begin{tabular}{|l|l|}
\hline Field & $61 \mathrm{~B}$ \\
\hline Crop history & 2019 corn, 2018 sorghum, 2017 wheat/sorghum \\
\hline Planting date & 31 Oct 2019 \\
\hline Variety & Shirley, Hilliard, Liberty 5658, Agrimaxx 463 \\
\hline Seeding rate & 30 seed/ft \\
\hline Plot length & $9^{\prime}$ \\
\hline Number of rows & 7 \\
\hline Row spacing & $6.67^{\prime}$ \\
\hline Alleys (length between blocks) & $9^{\prime}$ \\
\hline Harvest date & 10 Jun \\
\hline
\end{tabular}

EXPERIMENTAL DESIGN: Randomized complete block with four replicates

INOCULUM: Fusarium graminearum conidia $(\sim 50,000 / \mathrm{ml})$ applied 24 hours following the Feekes 10.5 .1 fungicide treatment with Lee Spider Sprayer; $1 \mathrm{~L}$ inoculum to $11 \mathrm{~L} \mathrm{H}_{2} \mathrm{O}$

TREATMENT APPLICATION:

\begin{tabular}{|l|l|}
\hline Equipment & Backpack sprayer \\
\hline Pressure (psi) & $38 \mathrm{psi}$ \\
\hline Nozzle type & Twinjet 8002VS \\
\hline Volume (gal/A) & 19.88 \\
\hline Surfactant & NIS $0.125 \% \mathrm{v} / \mathrm{v}$ \\
\hline
\end{tabular}

TREATMENTS:

\begin{tabular}{|l|l|l|l|l|l|}
\hline Trt \# & Variety & Fungicide and formulation & Rate, fl oz/A & App. timing & App. date \\
\hline 1 & Shirley & $\begin{array}{l}\text { Untreated } \\
\text { Inoculum }\end{array}$ & -- & $\begin{array}{l}-- \\
\text { Feekes 10.5.1 +1d }\end{array}$ & $17 \mathrm{Apr}$ \\
\hline 2 & Shirley & $\begin{array}{l}\text { Prosaro 421 SC } \\
\text { Inoculum }\end{array}$ & 6.5 & $\begin{array}{l}\text { Feekes 10.5.1 } \\
\text { Feekes 10.5.1+1d }\end{array}$ & $\begin{array}{l}16 \mathrm{Apr} \\
17 \mathrm{Apr}\end{array}$ \\
\hline 3 & Shirley & $\begin{array}{l}\text { Miravis Ace } \\
\text { Inoculum }\end{array}$ & 13.7 & $\begin{array}{l}\text { Feekes 10.5.1 } \\
\text { Feekes 10.5.1 +1d }\end{array}$ & $\begin{array}{l}16 \mathrm{Apr} \\
17 \mathrm{Apr}\end{array}$ \\
\hline 4 & Shirley & $\begin{array}{l}\text { Miravis Ace } \\
\text { Inoculum }\end{array}$ & 13.7 & $\begin{array}{l}\text { Feekes 10.3 } \\
\text { Feekes 10.5.1 +1d }\end{array}$ & $\begin{array}{l}11 \mathrm{Apr} \\
17 \mathrm{Apr}\end{array}$ \\
\hline 5 & Shirley & $\begin{array}{l}\text { Miravis Ace } \\
\text { Inoculum } \\
\text { Tebuconazole 3.6 F }\end{array}$ & 13.7 & $\begin{array}{l}\text { Feekes 10.5.1 } \\
\text { Feekes 10.5.1 +1d } \\
\text { Feekes 10.5.1 +4-6d }\end{array}$ & $\begin{array}{l}16 \mathrm{Apr} \\
17 \mathrm{Apr} \\
21 \mathrm{Apr}\end{array}$ \\
\hline 6 & Shirley & Untreated (no inoc) & -- & -- & $\begin{array}{l}-- \\
\text { Feekes 10.5.1 +1d }\end{array}$ \\
\hline 7 & Hilliard & $\begin{array}{l}\text { Untreated } \\
\text { Inoculum }\end{array}$ & -- & $\begin{array}{l}\text { Feekes 10.5.1 } \\
\text { Feekes 10.5.1 +1d }\end{array}$ & $\begin{array}{l}16 \mathrm{Apr} \\
17 \mathrm{Apr}\end{array}$ \\
\hline 8 & Hilliard & $\begin{array}{l}\text { Prosaro 421 SC } \\
\text { Inoculum }\end{array}$ & 6.5 & $\begin{array}{l}\text { Feekes 10.5.1 } \\
\text { Feekes 10.5.1 +1d }\end{array}$ & $\begin{array}{l}16 \mathrm{Apr} \\
17 \mathrm{Apr}\end{array}$ \\
\hline 9 & Hilliard & $\begin{array}{l}\text { Miravis Ace } \\
\text { Inoculum }\end{array}$ & 13.7 & $\begin{array}{l}\text { Feekes 10.3 } \\
\text { Feekes 10.5.1 +1d }\end{array}$ & $\begin{array}{l}11 \mathrm{Apr} \\
17 \mathrm{Apr}\end{array}$ \\
\hline 10 & Hilliard & $\begin{array}{l}\text { Miravis Ace } \\
\text { Inoculum }\end{array}$ & 13.7 & & \\
\hline
\end{tabular}




\begin{tabular}{|c|c|c|c|c|c|}
\hline 11 & Hilliard & $\begin{array}{l}\text { Miravis Ace } \\
\text { Inoculum } \\
\text { Tebuconazole } 3.6 \mathrm{~F}\end{array}$ & $\begin{array}{l}13.7 \\
4\end{array}$ & $\begin{array}{l}\text { Feekes } 10.5 .1 \\
\text { Feekes } 10.5 .1+1 \mathrm{~d} \\
\text { Feekes } 10.5 .1+4-6 \mathrm{~d}\end{array}$ & $\begin{array}{l}16 \mathrm{Apr} \\
17 \mathrm{Apr} \\
21 \mathrm{Apr}\end{array}$ \\
\hline 12 & Hilliard & Untreated (no inoc) & -- & -- & \\
\hline 13 & Liberty 5658 & $\begin{array}{l}\text { Untreated } \\
\text { Inoculum }\end{array}$ & -- & $\begin{array}{l}-- \\
\text { Feekes } 10.5 .1+1 \mathrm{~d}\end{array}$ & $17 \mathrm{Apr}$ \\
\hline 14 & Liberty 5658 & $\begin{array}{l}\text { Prosaro 421 SC } \\
\text { Inoculum }\end{array}$ & 6.5 & $\begin{array}{l}\text { Feekes 10.5.1 } \\
\text { Feekes 10.5.1 + 1d }\end{array}$ & $\begin{array}{l}16 \mathrm{Apr} \\
17 \mathrm{Apr}\end{array}$ \\
\hline 15 & Liberty 5658 & $\begin{array}{l}\text { Miravis Ace } \\
\text { Inoculum }\end{array}$ & 13.7 & $\begin{array}{l}\text { Feekes 10.5.1 } \\
\text { Feekes 10.5.1 + 1d }\end{array}$ & $\begin{array}{l}16 \mathrm{Apr} \\
17 \mathrm{Apr}\end{array}$ \\
\hline 16 & Liberty 5658 & $\begin{array}{l}\text { Miravis Ace } \\
\text { Inoculum }\end{array}$ & 13.7 & $\begin{array}{l}\text { Feekes } 10.3 \\
\text { Feekes } 10.5 .1+1 \mathrm{~d}\end{array}$ & $\begin{array}{l}11 \mathrm{Apr} \\
17 \mathrm{Apr}\end{array}$ \\
\hline 17 & Liberty 5658 & $\begin{array}{l}\text { Miravis Ace } \\
\text { Inoculum } \\
\text { Tebuconazole } 3.6 \mathrm{~F}\end{array}$ & $\begin{array}{l}13.7 \\
4 \\
\end{array}$ & $\begin{array}{l}\text { Feekes } 10.5 .1 \\
\text { Feekes } 10.5 .1+1 \mathrm{~d} \\
\text { Feekes } 10.5 .1+4-6 \mathrm{~d}\end{array}$ & $\begin{array}{l}16 \mathrm{Apr} \\
17 \mathrm{Apr} \\
21 \mathrm{Apr}\end{array}$ \\
\hline 18 & Liberty 5658 & Untreated (no inoc) & -- & -- & \\
\hline 19 & Agrimaxx 463 & $\begin{array}{l}\text { Untreated } \\
\text { Inoculum }\end{array}$ & -- & Feekes $10.5 .1+1 \mathrm{~d}$ & $17 \mathrm{Apr}$ \\
\hline 20 & Agrimaxx 463 & $\begin{array}{l}\text { Prosaro } 421 \mathrm{SC} \\
\text { Inoculum }\end{array}$ & 6.5 & $\begin{array}{l}\text { Feekes } 10.5 .1 \\
\text { Feekes } 10.5 .1+1 \mathrm{~d}\end{array}$ & $\begin{array}{l}16 \mathrm{Apr} \\
17 \mathrm{Apr}\end{array}$ \\
\hline 21 & Agrimaxx 463 & $\begin{array}{l}\text { Miravis Ace } \\
\text { Inoculum }\end{array}$ & 13.7 & $\begin{array}{l}\text { Feekes } 10.5 .1 \\
\text { Feekes } 10.5 .1+1 \mathrm{~d}\end{array}$ & $\begin{array}{l}16 \mathrm{Apr} \\
17 \mathrm{Apr}\end{array}$ \\
\hline 22 & Agrimaxx 463 & $\begin{array}{l}\text { Miravis Ace } \\
\text { Inoculum }\end{array}$ & 13.7 & $\begin{array}{l}\text { Feekes } 10.3 \\
\text { Feekes } 10.5 .1+1 \mathrm{~d}\end{array}$ & $\begin{array}{l}11 \mathrm{Apr} \\
17 \mathrm{Apr}\end{array}$ \\
\hline 23 & Agrimaxx 463 & $\begin{array}{l}\text { Miravis Ace } \\
\text { Inoculum } \\
\text { Tebuconazole } 3.6 \mathrm{~F}\end{array}$ & $\begin{array}{l}13.7 \\
4 \\
\end{array}$ & $\begin{array}{l}\text { Feekes } 10.5 .1 \\
\text { Feekes } 10.5 .1+1 \mathrm{~d} \\
\text { Feekes } 10.5 .1+4-6 \mathrm{~d}\end{array}$ & $\begin{array}{l}16 \mathrm{Apr} \\
17 \mathrm{Apr} \\
21 \mathrm{Apr}\end{array}$ \\
\hline 24 & Agrimaxx 463 & Untreated (no inoc) & -- & -- & \\
\hline
\end{tabular}

SOIL TYPE: Rains fine sandy loam

SOIL FERTILITY REPORT (Oct 2019):

\begin{tabular}{|l|c|c|c|c|c|c|c|c|c|}
\hline $\mathbf{p H}$ & $\mathbf{P}(\mathbf{l b} / \mathbf{A})$ & $\mathbf{K}(\mathbf{l b} / \mathbf{A})$ & $\mathbf{C a}(\mathbf{l b} / \mathbf{A})$ & $\mathbf{M g}(\mathbf{l b} / \mathbf{A})$ & $\mathbf{Z n}(\mathbf{p p m})$ & $\mathbf{M n}(\mathbf{p p m})$ & $\mathbf{C u}(\mathbf{p p m})$ & $\mathbf{F e}(\mathbf{p p m})$ & $\mathbf{B}(\mathbf{p p m})$ \\
\hline 5.3 & 178 & 377 & 1335 & 168 & 1.4 & 7.2 & 0.2 & 29.5 & 0.2 \\
\hline
\end{tabular}

\section{MAINTENANCE CHEMICAL PROGRAMS:}

\begin{tabular}{|l|l|}
\hline Fertilizer & Standard \\
\hline Herbicides & Standard \\
\hline Insecticides & Standard \\
\hline Fungicides & None except treatments \\
\hline Nematicides & None \\
\hline
\end{tabular}

MAINTENANCE CHEMICAL APPLICATIONS:

\begin{tabular}{|l|l|l|l|}
\hline Date & Type and target & Product and formulation & Rate/A \\
\hline $1-30-20$ & Fertility & $9-18-31$ & $322 \mathrm{lbs}$ \\
\hline $2-4-20$ & Fertility & $24-0-0-3$ & 60 units \\
\hline $3-14-20$ & Fertility & $24-0-0-3$ & 60 units \\
\hline $2-4-20$ & Herbicide & Quelex & $0.75 \mathrm{fl} \mathrm{oz}$ \\
\hline
\end{tabular}


Table 1. Effect of fungicide treatment and variety on disease incidence in wheat (WHTSCAB120, Suffolk, VA 2020).

\begin{tabular}{|c|c|c|c|c|c|}
\hline \multirow{2}{*}{\multicolumn{2}{|c|}{$\begin{array}{l}\text { Variety, treatment, rate/A } \\
\text { and application timing }(\text { Feekes })^{z}\end{array}$}} & \multicolumn{2}{|c|}{$\begin{array}{l}\text { \% leaf rust } \\
8 \text { May }\end{array}$} & \multicolumn{2}{|c|}{$\begin{array}{l}\text { Scab } \\
12 \text { May }\end{array}$} \\
\hline & & \multirow{2}{*}{ Flag } & \multirow{2}{*}{$\begin{array}{c}\text { Flag-1 } \\
5.0 \mathrm{c}-\mathrm{f}\end{array}$} & \multirow{2}{*}{$\frac{\% \text { severity }^{\mathrm{x}}}{0.0}$} & \multirow{2}{*}{$\begin{array}{c}\% \\
\text { incidence } \\
0.0 \mathrm{~d} \\
\end{array}$} \\
\hline 1. Shirley & Untreated & & & & \\
\hline 2. Shirley & Prosaro 421 SC $6.5 \mathrm{fl} \mathrm{oz}(\mathrm{F} 10.5 .1)$ & $0.3 \mathrm{de}$ & $5.0 \mathrm{c}-\mathrm{f}$ & 0.3 & $0.5 \mathrm{~d}$ \\
\hline 3. Shirley & Miravis Ace $13.7 \mathrm{fl} \mathrm{oz}(\mathrm{F} 10.5 .1)$ & $0.0 \mathrm{e}$ & $2.8 \mathrm{~g}$ & 0.3 & $2.3 \mathrm{a}-\mathrm{d}$ \\
\hline 4. Shirley & Miravis Ace $13.7 \mathrm{fl} \mathrm{oz}(\mathrm{F} 10.3)$ & $0.0 \mathrm{e}$ & $4.0 \mathrm{e}-\mathrm{g}$ & 0.0 & $0.0 \mathrm{~d}$ \\
\hline 5. Shirley & $\begin{array}{l}\text { Miravis Ace } 13.7 \mathrm{fl} \mathrm{oz}(\mathrm{F} 10.5 .1) \\
\text { Tebuconazole } 3.6 \mathrm{~F} 4.0 \mathrm{fl} \mathrm{oz}(\mathrm{F} 10.5 .1+4-6 \mathrm{~d})\end{array}$ & $0.3 \mathrm{de}$ & $4.0 \mathrm{e}-\mathrm{g}$ & 0.0 & $0.0 \mathrm{~d}$ \\
\hline 6. Shirley & Untreated (no inoculum) & $0.5 \mathrm{c}-\mathrm{e}$ & $5.0 \mathrm{c}-\mathrm{f}$ & 0.0 & $0.0 \mathrm{~d}$ \\
\hline 7. Hilliard & Untreated & $1.5 \mathrm{~cd}$ & $6.3 \mathrm{c}-\mathrm{e}$ & 0.3 & $4.0 \mathrm{a}-\mathrm{c}$ \\
\hline 8. Hilliard & Prosaro 421 SC $6.5 \mathrm{fl} \mathrm{oz} \mathrm{(F10.5.1)}$ & $0.8 \mathrm{c}-\mathrm{e}$ & $5.0 \mathrm{c}-\mathrm{f}$ & 0.3 & $2.8 \mathrm{a}-\mathrm{d}$ \\
\hline 9. Hilliard & Miravis Ace $13.7 \mathrm{fl} \mathrm{oz}(\mathrm{F} 10.5 .1)$ & $0.5 \mathrm{c}-\mathrm{e}$ & $4.0 \mathrm{e}-\mathrm{g}$ & 0.0 & $0.0 \mathrm{~d}$ \\
\hline 10. Hilliard & Miravis Ace $13.7 \mathrm{fl} \mathrm{oz}(\mathrm{F} 10.3)$ & $1.8 \mathrm{bc}$ & $5.3 \mathrm{~d}-\mathrm{g}$ & 0.0 & $0.0 \mathrm{~d}$ \\
\hline 11. Hilliard & $\begin{array}{l}\text { Miravis Ace } 13.7 \mathrm{fl} \mathrm{oz}(\mathrm{F} 10.5 .1) \\
\text { Tebuconazole } 3.6 \mathrm{~F} 4.0 \mathrm{fl} \mathrm{oz}(\mathrm{F} 10.5 .1+4-6 \mathrm{~d})\end{array}$ & $0.5 \mathrm{c}-\mathrm{e}$ & $4.0 \mathrm{e}-\mathrm{g}$ & 0.0 & $0.8 \mathrm{~cd}$ \\
\hline 12. Hilliard & Untreated (no inoculum) & $0.5 \mathrm{c}-\mathrm{e}$ & $3.0 \mathrm{fg}$ & 0.3 & $1.5 \mathrm{a}-\mathrm{d}$ \\
\hline 13. Liberty 5658 & Untreated & $3.8 \mathrm{ab}$ & $10.3 \mathrm{a}-\mathrm{c}$ & 0.5 & $4.8 \mathrm{ab}$ \\
\hline 14. Liberty 5658 & Prosaro 421 SC $6.5 \mathrm{fl} \mathrm{oz}(\mathrm{F} 10.5 .1)$ & $3.8 \mathrm{ab}$ & $7.5 \mathrm{~b}-\mathrm{e}$ & 0.0 & $0.8 \mathrm{~cd}$ \\
\hline 15. Liberty 5658 & Miravis Ace $13.7 \mathrm{fl} \mathrm{oz}(\mathrm{F} 10.5 .1)$ & $5.0 \mathrm{a}$ & $13.8 \mathrm{a}$ & 0.3 & $3.3 \mathrm{a}-\mathrm{d}$ \\
\hline 16. Liberty 5658 & Miravis Ace $13.7 \mathrm{fl} \mathrm{oz}(\mathrm{F} 10.3)$ & $3.0 \mathrm{ab}$ & $8.8 \mathrm{~b}-\mathrm{d}$ & 0.0 & $1.5 \mathrm{a}-\mathrm{d}$ \\
\hline 17. Liberty 5658 & $\begin{array}{l}\text { Miravis Ace } 13.7 \mathrm{fl} \mathrm{oz}(\mathrm{F} 10.5 .1) \\
\text { Tebuconazole } 3.6 \mathrm{~F} 4.0 \mathrm{fl} \mathrm{oz}(\mathrm{F} 10.5 .1+4-6 \mathrm{~d})\end{array}$ & $5.0 \mathrm{a}$ & $13.8 \mathrm{a}$ & 0.0 & $3.8 \mathrm{a}-\mathrm{c}$ \\
\hline 18. Liberty 5658 & Untreated (no inoculum) & $5.0 \mathrm{a}$ & $11.3 \mathrm{ab}$ & 0.0 & $0.0 \mathrm{~d}$ \\
\hline 19. Agrimaxx 463 & Untreated & $0.0 \mathrm{e}$ & $0.3 \mathrm{~h}$ & 0.0 & $1.3 \mathrm{~b}-\mathrm{d}$ \\
\hline 20. Agrimaxx 463 & Prosaro 421 SC $6.5 \mathrm{fl} \mathrm{oz} \mathrm{(F10.5.1)}$ & $0.0 \mathrm{e}$ & $0.0 \mathrm{~h}$ & 0.5 & $5.8 \mathrm{a}$ \\
\hline 21. Agrimaxx 463 & Miravis Ace $13.7 \mathrm{fl} \mathrm{oz}(\mathrm{F} 10.5 .1)$ & $0.0 \mathrm{e}$ & $0.0 \mathrm{~h}$ & 0.3 & $3.0 \mathrm{a}-\mathrm{d}$ \\
\hline 22. Agrimaxx 463 & Miravis Ace $13.7 \mathrm{fl} \mathrm{oz}(\mathrm{F} 10.3)$ & $0.0 \mathrm{e}$ & $0.0 \mathrm{~h}$ & 0.0 & $0.8 \mathrm{~cd}$ \\
\hline 23. Agrimaxx 463 & $\begin{array}{l}\text { Miravis Ace } 13.7 \mathrm{fl} \mathrm{oz}(\mathrm{F} 10.5 .1) \\
\text { Tebuconazole } 3.6 \mathrm{~F} 4.0 \mathrm{fl} \mathrm{oz}(\mathrm{F} 10.5 .1+4-6 \mathrm{~d})\end{array}$ & $0.0 \mathrm{e}$ & $0.3 \mathrm{~h}$ & 0.0 & $0.0 \mathrm{~d}$ \\
\hline 24. Agrimaxx 463 & Untreated (no inoculum) & $0.0 \mathrm{e}$ & $0.0 \mathrm{~h}$ & 0.0 & $0.0 \mathrm{~d}$ \\
\hline$P(\mathrm{~F})$ & & $<0.0001$ & $<0.0001$ & 0.52 & 0.01 \\
\hline LSD & & 1.62 & 3.46 & N.S. & 3.98 \\
\hline
\end{tabular}

z Fungicide sprays were applied at Feekes 10.3 on 11 Apr, Feekes 10.5.1 (flowering) on 16 Apr, and Feekes 10.5.1 + 4-6d on 21 Apr. Fusarium inoculum (trts 1-5) was applied hours after F10.5 treatment application (17 Apr). All fungicide treatments were applied with Induce $0.125 \% \mathrm{v} / \mathrm{v}$.

y Percent of leaf area with signs and symptoms of Puccinia recondita f. sp. Tritici. Plots were also evaluated for powdery mildew and Stagnospora nodorum blotch; little to none was detected.

$x$ Percent of grain heads with signs and symptoms of Fusarium head blight.

w Percent of spikelets with signs and symptoms of Fusarium head blight.

Means in a column or group followed by the same letter(s) are not significantly different according to Fisher's Protected LSD $(P=0.05)$. Arcsine transformation of percentage data was made in analysis to determine statistical significance. 
Table 2. Effect of fungicide treatment and variety on yield and mycotoxin levels in wheat (WHTSCAB120, Suffolk, VA 2020).

\begin{tabular}{|c|c|c|c|c|c|}
\hline \multicolumn{2}{|c|}{$\begin{array}{l}\text { Variety, treatment, rate/A } \\
\text { and application timing (Feekes) }{ }^{\mathrm{z}}\end{array}$} & \multirow{2}{*}{$\begin{array}{c}\text { Yield }^{\mathrm{y}} \\
\text { (bu/A) }^{\text {bu/A }}\end{array}$} & \multirow{2}{*}{$\begin{array}{c}\begin{array}{c}\text { Test weight } \\
\text { (lb/bu) }\end{array} \\
54.5 \text { ef } \\
\end{array}$} & \multirow{2}{*}{$\frac{\% \text { FDK }^{\mathbf{x}}}{3.0}$} & \multirow{2}{*}{$\begin{array}{c}\begin{array}{c}\text { DON } \\
\text { ppm }\end{array} \\
0.9 \mathrm{bc} \\
\end{array}$} \\
\hline 1. Shirley & Untreated & & & & \\
\hline 2. Shirley & Prosaro 421 SC $6.5 \mathrm{fl} \mathrm{oz}(\mathrm{F} 10.5 .1)$ & 52.7 & $54.2 \mathrm{e}-\mathrm{g}$ & 2.3 & $0.8 \mathrm{~b}-\mathrm{c}$ \\
\hline 3. Shirley & Miravis Ace $13.7 \mathrm{fl} \mathrm{oz}(\mathrm{F} 10.5 .1)$ & 62.7 & $52.7 \mathrm{~g}$ & 0.8 & $0.2 \mathrm{f}-\mathrm{i}$ \\
\hline 4. Shirley & Miravis Ace $13.7 \mathrm{fl} \mathrm{oz}(\mathrm{F} 10.3)$ & 51.8 & $55.4 \mathrm{c}-\mathrm{e}$ & 1.3 & $0.5 \mathrm{~d}-\mathrm{h}$ \\
\hline 5. Shirley & $\begin{array}{l}\text { Miravis Ace } 13.7 \mathrm{fl} \mathrm{oz}(\mathrm{F} 10.5 .1) \\
\text { Tebuconazole } 3.6 \mathrm{~F} 4.0 \mathrm{fl} \mathrm{oz}(\mathrm{F} 10.5 .1+4-6 \mathrm{~d})\end{array}$ & 55.5 & $56.5 \mathrm{a}-\mathrm{c}$ & 1.5 & $0.3 \mathrm{e}-\mathrm{i}$ \\
\hline 6. Shirley & Untreated (no inoculum) & 47.8 & $54.1 \mathrm{e}-\mathrm{g}$ & 2.0 & $0.5 \mathrm{c}-\mathrm{h}$ \\
\hline 7. Hilliard & Untreated & 50.7 & $57.1 \mathrm{ab}$ & 1.3 & $0.9 \mathrm{~b}$ \\
\hline 8. Hilliard & Prosaro 421 SC $6.5 \mathrm{fl} \mathrm{oz} \mathrm{(F10.5.1)}$ & 60.0 & $57.1 \mathrm{ab}$ & 2.5 & $0.6 \mathrm{~b}-\mathrm{f}$ \\
\hline 9. Hilliard & Miravis Ace $13.7 \mathrm{fl} \mathrm{oz}(\mathrm{F} 10.5 .1)$ & 59.8 & $56.8 \mathrm{a}-\mathrm{c}$ & 0.8 & $0.3 \mathrm{e}-\mathrm{i}$ \\
\hline 10. Hilliard & Miravis Ace $13.7 \mathrm{fl} \mathrm{oz}(\mathrm{F} 10.3)$ & 57.4 & $57.6 \mathrm{a}$ & 1.8 & $0.6 \mathrm{~b}-\mathrm{f}$ \\
\hline 11. Hilliard & $\begin{array}{l}\text { Miravis Ace } 13.7 \mathrm{fl} \mathrm{oz}(\mathrm{F} 10.5 .1) \\
\text { Tebuconazole } 3.6 \mathrm{~F} 4.0 \mathrm{fl} \mathrm{oz}(\mathrm{F} 10.5 .1+4-6 \mathrm{~d})\end{array}$ & 49.4 & $57.5 \mathrm{a}$ & 1.3 & $0.6 \mathrm{~b}-\mathrm{f}$ \\
\hline 12. Hilliard & Untreated (no inoculum) & 58.2 & $57.1 \mathrm{a}$ & 1.5 & $0.5 \mathrm{~d}-\mathrm{h}$ \\
\hline 13. Liberty 5658 & Untreated & 56.6 & $56.3 \mathrm{a}-\mathrm{d}$ & 2.3 & $1.4 \mathrm{a}$ \\
\hline 14. Liberty 5658 & Prosaro 421 SC $6.5 \mathrm{fl} \mathrm{oz} \mathrm{(F10.5.1)}$ & 68.6 & $57.1 \mathrm{ab}$ & 1.5 & $0.5 \mathrm{~b}-\mathrm{g}$ \\
\hline 15. Liberty 5658 & Miravis Ace $13.7 \mathrm{fl} \mathrm{oz}(\mathrm{F} 10.5 .1)$ & 57.1 & $57.5 \mathrm{a}$ & 1.5 & $0.6 \mathrm{~b}-\mathrm{e}$ \\
\hline 16. Liberty 5658 & Miravis Ace $13.7 \mathrm{fl} \mathrm{oz}(\mathrm{F} 10.3)$ & 61.6 & $57.2 \mathrm{a}$ & 1.5 & $0.9 \mathrm{~b}$ \\
\hline 17. Liberty 5658 & $\begin{array}{l}\text { Miravis Ace } 13.7 \mathrm{fl} \mathrm{oz}(\mathrm{F} 10.5 .1) \\
\text { Tebuconazole } 3.6 \mathrm{~F} 4.0 \mathrm{fl} \mathrm{oz}(\mathrm{F} 10.5 .1+4-6 \mathrm{~d})\end{array}$ & 57.8 & $57.6 \mathrm{a}$ & 0.8 & $0.7 \mathrm{~b}-\mathrm{c}$ \\
\hline 18. Liberty 5658 & Untreated (no inoculum) & 62.9 & $56.9 \mathrm{a}-\mathrm{c}$ & 1.5 & $0.5 \mathrm{c}-\mathrm{h}$ \\
\hline 19. Agrimaxx 463 & Untreated & 60.1 & $53.6 \mathrm{fg}$ & 1.3 & $0.1 \mathrm{hi}$ \\
\hline 20. Agrimaxx 463 & Prosaro 421 SC $6.5 \mathrm{fl} \mathrm{oz} \mathrm{(F10.5.1)}$ & 57.8 & $54.9 \mathrm{~d}-\mathrm{f}$ & 1.3 & $0.0 \mathrm{i}$ \\
\hline 21. Agrimaxx 463 & Miravis Ace $13.7 \mathrm{fl} \mathrm{oz}(\mathrm{F} 10.5 .1)$ & 59.8 & $54.8 \mathrm{~d}-\mathrm{f}$ & 1.0 & $0.2 \mathrm{~g}-\mathrm{i}$ \\
\hline 22. Agrimaxx 463 & Miravis Ace $13.7 \mathrm{fl} \mathrm{oz}(\mathrm{F} 10.3)$ & 46.6 & $55.5 \mathrm{~b}-\mathrm{e}$ & 1.3 & $0.1 \mathrm{i}$ \\
\hline 23. Agrimaxx 463 & $\begin{array}{l}\text { Miravis Ace } 13.7 \mathrm{fl} \mathrm{oz}(\mathrm{F} 10.5 .1) \\
\text { Tebuconazole } 3.6 \mathrm{~F} 4.0 \mathrm{fl} \mathrm{oz}(\mathrm{F} 10.5 .1+4-6 \mathrm{~d})\end{array}$ & 56.1 & $54.8 \mathrm{~d}-\mathrm{f}$ & 0.3 & $0.1 \mathrm{i}$ \\
\hline 24. Agrimaxx 463 & Untreated (no inoculum) & 52.6 & $54.9 \mathrm{~d}-\mathrm{f}$ & 1.3 & $0.1 \mathrm{i}$ \\
\hline$P(\mathrm{~F})$ & & 0.92 & $<0.0001$ & 0.50 & $<0.0001$ \\
\hline LSD & & N.S. & 1.59 & N.S. & 0.37 \\
\hline
\end{tabular}

z Fungicide sprays were applied at Feekes 10.3 on 11 Apr, Feekes 10.5.1 (flowering) on 16 Apr, and Feekes 10.5.1 + 4-6d on 21 Apr. Fusarium inoculum (trts 1-5) was applied hours after F10.5 treatment application (17 Apr). All fungicide treatments were applied with Induce $0.125 \% \mathrm{v} / \mathrm{v}$.

y Yields are weight of wheat with $13.5 \%$ moisture. One bushel equals $60 \mathrm{lbs}$. Wheat was harvested on $10 \mathrm{Jun}$.

$x$ Percent Fusarium damaged kernels. FDK rating scale: $0=$ no damage, 100=100\% Fusarium damaged kernels in scabby wheat; FDK scale by Engle, De Wolf \& Lipps, Ohio State.

Means in a column or group followed by the same letter(s) are not significantly different according to Fisher's Protected LSD $(P=0.05)$. Arcsine transformation of percentage data was made in analysis to determine statistical significance. 
TEST ID: WHTSCAB220

PURPOSE: To evaluate fungicide treatment and application timing for control of FHB and impact on yield in wheat

LOCATION: Tidewater Research Farm, Hare Rd., Suffolk, VA

CROP INFORMATION:

\begin{tabular}{|l|l|}
\hline Field & $9 \mathrm{~B}$ \\
\hline Crop history & 2019 wheat/soy, 2018 corn, 2017 wheat/soy \\
\hline Planting date & 22 Nov 2019 \\
\hline Variety & Shirley \\
\hline Seeding rate & 30 seed/ft \\
\hline Plot length/width & $30^{\prime} \times 5^{\prime}$ (treat \& harvest), 30' x 12' total \\
\hline Number of rows & 8 \\
\hline Row spacing & $7.5^{\prime \prime}$ \\
\hline Alleys (length between blocks) & $9^{\prime}$ \\
\hline Harvest date & $22 \mathrm{Jun}$ \\
\hline
\end{tabular}

EXPERIMENTAL DESIGN: Randomized complete block with four replicates

INOCULUM: Fusarium graminearum conidia $(\sim 50,000 / \mathrm{ml})$ applied 24 hours following the Feekes 10.5 .1 fungicide treatment with Lee Spider Sprayer; $1 \mathrm{~L}$ inoculum to $11 \mathrm{~L} \mathrm{H}_{2} \mathrm{O}$

TREATMENT APPLICATION:

\begin{tabular}{|l|l|}
\hline Equipment & Lee Spider sprayer \\
\hline Pressure (psi) & $38 \mathrm{psi}$ \\
\hline Nozzle type & Twinjet $8002 \mathrm{VS}$ \\
\hline Volume (gal/A) & 19.88 \\
\hline Surfactant & NIS $0.125 \% \mathrm{v} / \mathrm{v}$ \\
\hline
\end{tabular}

TREATMENTS:

\begin{tabular}{|c|c|c|c|c|}
\hline Trt \# & Fungicide and formulation & Rate, fl oz/A & App. timing & App. date \\
\hline 1 & $\begin{array}{l}\text { Untreated } \\
\text { Inoculum }\end{array}$ & - & $\begin{array}{l}-- \\
\text { Feekes } 10.5 .1+1 d\end{array}$ & $29 \mathrm{Apr}$ \\
\hline 2 & $\begin{array}{l}\text { Prosaro 421 SC } \\
\text { Inoculum }\end{array}$ & 6.5 & $\begin{array}{l}\text { Feekes 10.5.1 } \\
\text { Feekes } 10.5 .1+1 \mathrm{~d}\end{array}$ & $\begin{array}{l}28 \mathrm{Apr} \\
29 \mathrm{Apr}\end{array}$ \\
\hline 3 & $\begin{array}{l}\text { Caramba } 0.75 \mathrm{SL} \\
\text { Inoculum }\end{array}$ & 13.5 & $\begin{array}{l}\text { Feekes } 10.5 .1 \\
\text { Feekes } 10.5 .1+1 \mathrm{~d}\end{array}$ & $\begin{array}{l}28 \mathrm{Apr} \\
29 \mathrm{Apr}\end{array}$ \\
\hline 4 & $\begin{array}{l}\text { Miravis Ace SE } \\
\text { Inoculum }\end{array}$ & 13.7 & $\begin{array}{l}\text { Feekes } 10.3 \\
\text { Feekes } 10.5 .1+1 \mathrm{~d}\end{array}$ & $\begin{array}{l}21 \mathrm{Apr} \\
29 \mathrm{Apr}\end{array}$ \\
\hline 5 & $\begin{array}{l}\text { Miravis Ace SE } \\
\text { Inoculum }\end{array}$ & 13.7 & $\begin{array}{l}\text { Feekes 10.5.1 } \\
\text { Feekes 10.5.1 +1d }\end{array}$ & $\begin{array}{l}28 \mathrm{Apr} \\
29 \mathrm{Apr}\end{array}$ \\
\hline 6 & $\begin{array}{l}\text { Miravis Ace SE } \\
\text { Inoculum }\end{array}$ & 13.7 & $\begin{array}{l}\text { Feekes } 10.5 .1+4-6 \mathrm{~d} \\
\text { Feekes } 10.5 .1+1 \mathrm{~d}\end{array}$ & $\begin{array}{l}\text { 4 May } \\
29 \mathrm{Apr}\end{array}$ \\
\hline 7 & $\begin{array}{l}\text { Miravis Ace SE } \\
\text { Inoculum } \\
\text { Prosaro } 421 \mathrm{SC}\end{array}$ & $\begin{array}{l}13.7 \\
6.5\end{array}$ & $\begin{array}{l}\text { Feekes } 10.5 .1 \\
\text { Feekes } 10.5 .1+1 \mathrm{~d} \\
\text { Feekes } 10.5 .1+4-6 \mathrm{~d}\end{array}$ & $\begin{array}{l}28 \mathrm{Apr} \\
29 \mathrm{Apr} \\
4 \mathrm{May}\end{array}$ \\
\hline 8 & $\begin{array}{l}\text { Miravis Ace SE } \\
\text { Inoculum } \\
\text { Caramba } 0.75 \mathrm{SL}\end{array}$ & $\begin{array}{l}13.7 \\
13.5 \\
\end{array}$ & $\begin{array}{l}\text { Feekes } 10.5 .1 \\
\text { Feekes } 10.5 .1+1 \mathrm{~d} \\
\text { Feekes } 10.5 .1+4-6 \mathrm{~d}\end{array}$ & $\begin{array}{l}28 \mathrm{Apr} \\
29 \mathrm{Apr} \\
4 \mathrm{May}\end{array}$ \\
\hline 9 & $\begin{array}{l}\text { Miravis Ace SE } \\
\text { Inoculum } \\
\text { Tebuconazole } 3.6 \mathrm{~F}\end{array}$ & $\begin{array}{l}13.7 \\
4 \\
\end{array}$ & $\begin{array}{l}\text { Feekes } 10.5 .1 \\
\text { Feekes } 10.5 .1+1 \mathrm{~d} \\
\text { Feekes } 10.5 .1+4-6 \mathrm{~d}\end{array}$ & $\begin{array}{l}28 \mathrm{Apr} \\
29 \mathrm{Apr} \\
4 \mathrm{May}\end{array}$ \\
\hline
\end{tabular}




\begin{tabular}{|l|l|l|l|l|}
\hline 10 & $\begin{array}{l}\text { Prosaro 421 SC } \\
\text { Inoculum }\end{array}$ & 6.5 & $\begin{array}{l}\text { Feekes 10.3 } \\
\text { Feekes 10.5.1 +1d }\end{array}$ & $\begin{array}{l}21 \mathrm{Apr} \\
29 \mathrm{Apr}\end{array}$ \\
\hline 11 & $\begin{array}{l}\text { Prosaro 421 SC } \\
\text { Inoculum }\end{array}$ & 6.5 & $\begin{array}{l}\text { Feekes 10.5.1+4-6d } \\
\text { Feekes 10.5.1 +1d }\end{array}$ & $\begin{array}{l}4 \mathrm{May} \\
29 \mathrm{Apr}\end{array}$ \\
\hline 12 & $\begin{array}{l}\text { Caramba 0.75 SL } \\
\text { Inoculum }\end{array}$ & 13.5 & $\begin{array}{l}\text { Feekes 10.3 } \\
\text { Feekes 10.5.1 +1d }\end{array}$ & $\begin{array}{l}21 \mathrm{Apr} \\
29 \mathrm{Apr}\end{array}$ \\
\hline 13 & $\begin{array}{l}\text { Caramba 0.75 SL } \\
\text { Inoculum }\end{array}$ & 13.5 & $\begin{array}{l}\text { Feekes 10.5.1 +4-6d } \\
\text { Feekes 10.5.1 +1d }\end{array}$ & $\begin{array}{l}4 \mathrm{May} \\
29 \mathrm{Apr}\end{array}$ \\
\hline
\end{tabular}

SOIL TYPE: Suffolk loamy sand

SOIL FERTILITY REPORT (Oct 2019):

\begin{tabular}{|l|c|c|c|c|c|c|c|c|c|}
\hline $\mathbf{p H}$ & $\mathbf{P}(\mathbf{l b} / \mathbf{A})$ & $\mathbf{K}(\mathbf{l b} / \mathbf{A})$ & $\mathbf{C a}(\mathbf{l b} / \mathbf{A})$ & $\mathbf{M g}(\mathbf{l b} / \mathbf{A})$ & $\mathbf{Z n}(\mathbf{p p m})$ & $\mathbf{M n}(\mathbf{p p m})$ & $\mathbf{C u}(\mathbf{p p m})$ & $\mathbf{F e}(\mathbf{p p m})$ & $\mathbf{B}(\mathbf{p p m})$ \\
\hline 5.7 & 102 & 50 & 642 & 92 & 0.6 & 4.6 & 0.4 & 19.3 & 0.1 \\
\hline
\end{tabular}

MAINTENANCE CHEMICAL PROGRAMS:

\begin{tabular}{|l|l|}
\hline Fertilizer & Standard \\
\hline Herbicides & Standard \\
\hline Insecticides & Standard \\
\hline Fungicides & None except treatments \\
\hline Nematicides & None \\
\hline
\end{tabular}

MAINTENANCE CHEMICAL APPLICATIONS:

\begin{tabular}{|l|l|l|l|}
\hline Date & Type and target & Product and formulation & Rate/A \\
\hline $1-30-20$ & Fertility & $9-18-31$ & $322 \mathrm{lbs}$ \\
\hline $2-4-20$ & Fertility & $24-0-0-3$ & 60 units \\
\hline $3-14-20$ & Fertility & $24-0-0-3$ & 60 units \\
\hline $2-4-20$ & Herbicide & Quelex & $0.75 \mathrm{fl} \mathrm{oz}$ \\
\hline
\end{tabular}


Table 1. Effect of fungicide treatment on disease incidence in wheat (WHTSCAB220, Suffolk, VA 2020).

\begin{tabular}{|c|c|c|c|c|c|}
\hline \multirow{3}{*}{$\begin{array}{l}\text { Treatment, rate/A } \\
\text { and application timing }\left(\text { Feekes) }{ }^{\mathrm{z}}\right.\end{array}$} & \multicolumn{5}{|c|}{$\begin{array}{l}\text { \% foliar disease } \\
20 \text { May }\end{array}$} \\
\hline & \multirow{2}{*}{$\begin{array}{c}\begin{array}{c}\text { Leaf } \\
\text { blotch }^{y}\end{array} \\
\text { Flag-1 } \\
\end{array}$} & \multicolumn{2}{|c|}{ Leaf rust ${ }^{\mathrm{x}}$} & \multicolumn{2}{|c|}{ Stripe rust ${ }^{w}$} \\
\hline & & Flag & Flag-1 & Flag & Flag-1 \\
\hline 1. Untreated & $18.8 \mathrm{a}$ & 0.0 & 5.0 & 2.0 & 11.3 \\
\hline 2. Prosaro 421 SC $6.5 \mathrm{fl} \mathrm{oz} \mathrm{(F10.5.1)}$ & $5.0 \mathrm{~b}-\mathrm{d}$ & 0.0 & 5.0 & 0.3 & 2.5 \\
\hline 3. Caramba 0.75 SL $13.5 \mathrm{fl} \mathrm{oz} \mathrm{(F10.5.1)}$ & 1.0 ef & 1.3 & 10.3 & 0.0 & 0.0 \\
\hline 4. Miravis ACE SE $13.7 \mathrm{fl} \mathrm{oz} \mathrm{(F10.3)}$ & $0.8 \mathrm{f}$ & 0.0 & 4.0 & 0.0 & 0.0 \\
\hline 5. Miravis ACE SE $13.7 \mathrm{fl} \mathrm{oz}(\mathrm{F} 10.5 .1)$ & $3.0 \mathrm{c}-\mathrm{f}$ & 0.0 & 10.0 & 0.5 & 1.3 \\
\hline 6. Miravis ACE SE $13.7 \mathrm{fl}$ oz (F10.5.1 + 4-6d) & $2.0 \mathrm{~d}-\mathrm{f}$ & 0.3 & 6.3 & 0.3 & 1.5 \\
\hline $\begin{array}{l}\text { 7. Miravis ACE SE } 13.7 \mathrm{fl} \mathrm{oz}(\mathrm{F} 10.5 .1) \\
\text { Prosaro 421 SC } 6.5 \mathrm{fl} \mathrm{oz}(\mathrm{F} 10.5 .1+4-6 \mathrm{~d}) \\
\end{array}$ & $4.3 \mathrm{~b}-\mathrm{e}$ & 0.0 & 7.5 & 0.3 & 1.3 \\
\hline $\begin{array}{l}\text { 8. Miravis ACE SE } 13.7 \mathrm{fl} \mathrm{oz}(\mathrm{F} 10.5 .1) \\
\text { Caramba 0.75 SL } 13.5 \mathrm{fl} \mathrm{oz}(\mathrm{F} 10.5 .1+4-6 \mathrm{~d})\end{array}$ & $3.0 \mathrm{c}-\mathrm{f}$ & 0.3 & 5.3 & 1.3 & 5.0 \\
\hline $\begin{array}{ll}\text { 9. } & \text { Miravis ACE SE } 13.7 \mathrm{fl} \mathrm{oz}(\mathrm{F} 10.5 .1) \\
& \text { Tebuconazole } 3.6 \mathrm{~F} 4 \mathrm{fl} \mathrm{oz}(\mathrm{F} 10.5 .1+4-6 \mathrm{~d}) \\
\end{array}$ & $2.0 \mathrm{~d}-\mathrm{f}$ & 1.3 & 10.0 & 0.0 & 0.0 \\
\hline 10. Prosaro 421 SC $6.5 \mathrm{fl} \mathrm{oz}(\mathrm{F} 10.3)$ & $7.8 \mathrm{~b}$ & 0.0 & 6.3 & 1.3 & 2.5 \\
\hline 11. Prosaro 421 SC $6.5 \mathrm{fl} \mathrm{oz}(\mathrm{F} 10.5 .1+4-6 \mathrm{~d})$ & $7.8 \mathrm{~b}$ & 0.0 & 10.0 & 0.0 & 0.3 \\
\hline 12. Caramba $0.75 \mathrm{SL} 13.5 \mathrm{fl} \mathrm{oz}$ (F10.3) & $3.0 \mathrm{c}-\mathrm{f}$ & 0.0 & 7.5 & 0.0 & 0.3 \\
\hline 13. Caramba 0.75 SL $13.5 \mathrm{fl} \mathrm{oz}(\mathrm{F} 10.5 .1+4-6 \mathrm{~d})$ & $6.3 \mathrm{bc}$ & 0.3 & 6.3 & 0.0 & 0.3 \\
\hline$P(\mathrm{~F})$ & $<0.0001$ & 0.71 & 0.34 & 0.08 & 0.11 \\
\hline LSD & 4.68 & N.S. & N.S. & N.S. & N.S. \\
\hline
\end{tabular}

z $\quad$ Fungicide sprays were applied at Feekes 3 on 21 Apr, Feekes 10.5.1 (flowering) on 28 Apr, and Feekes 10.5.1 + 4-6d on 4 May. Fusarium graminearum conidia $(\sim 50,000 / \mathrm{ml})$ applied on 29 Apr to all plots 24 hours following the Feekes 10.5.1 fungicide treatment. All fungicide treatments applied with Induce $0.125 \% \mathrm{v} / \mathrm{v}$.

y Percent of leaf area with signs and symptoms of Stagnospora nodorum blotch. Plots were also evaluated for symptoms of powdery mildew; none were observed.

$x$ Percent of leaf area with signs and symptoms of Puccinia recondite f. sp. tritici.

w Percent of leaf area with signs and symptoms of Puccinia striiformis f. sp. tritici.

Means in a column or group followed by the same letter(s) are not significantly different according to Fisher's Protected LSD $(P=0.05)$. Arcsine transformation of percentage data was made in analysis to determine statistical significance. 
Table 2. Effect of fungicide treatment on disease severity and yield in wheat (WHTSCAB220, Suffolk, VA 2020).

\begin{tabular}{|c|c|c|c|c|c|}
\hline \multirow[b]{2}{*}{$\begin{array}{l}\text { Treatment, rate/A } \\
\text { and application timing }(\text { Feekes })^{\mathrm{z}}\end{array}$} & \multicolumn{2}{|c|}{$\begin{array}{c}\text { Scab } \\
17 \text { May }\end{array}$} & \multirow[b]{2}{*}{$\begin{array}{l}\text { Yield } \\
\text { (bu/A) }\end{array}$} & \multirow[b]{2}{*}{$\begin{array}{c}\text { Test weight } \\
(\text { lb/bu) }\end{array}$} & \multirow[b]{2}{*}{$\%$ FDK $^{v}$} \\
\hline & $\begin{array}{c}\begin{array}{c}\text { \% seve- } \\
\text { rity }\end{array} \\
\end{array}$ & $\begin{array}{l}\% \text { inci- } \\
\text { dence }^{x}\end{array}$ & & & \\
\hline 1. Untreated & 0.2 & 2.4 & 90.5 & $54.5 \mathrm{~b}$ & 3.0 \\
\hline 2. Prosaro $421 \mathrm{SC} 6.5 \mathrm{fl} \mathrm{oz}(\mathrm{F} 10.5 .1)$ & 0.1 & 0.7 & 98.5 & $54.7 \mathrm{~b}$ & 2.0 \\
\hline 3. Caramba 0.75 SL $13.5 \mathrm{fl} \mathrm{oz} \mathrm{(F10.5.1)}$ & 0.1 & 1.7 & 84.7 & $54.5 \mathrm{~b}$ & 2.0 \\
\hline 4. Miravis ACE SE $13.7 \mathrm{fl} \mathrm{oz} \mathrm{(F10.3)}$ & 0.0 & 0.0 & 100.8 & $56.0 \mathrm{a}$ & 1.5 \\
\hline 5. Miravis ACE SE $13.7 \mathrm{fl} \mathrm{oz}(\mathrm{F} 10.5 .1)$ & 0.0 & 0.4 & 99.3 & $56.5 \mathrm{a}$ & 1.8 \\
\hline 6. Miravis ACE SE $13.7 \mathrm{fl} \mathrm{oz}(\mathrm{F} 10.5 .1+4-6 \mathrm{~d})$ & 0.2 & 2.2 & 87.8 & $56.1 \mathrm{a}$ & 2.3 \\
\hline $\begin{array}{ll}\text { 7. } & \text { Miravis ACE SE } 13.7 \mathrm{fl} \mathrm{oz} \mathrm{(F10.5.1)} \\
\text { Prosaro 421 SC } 6.5 \mathrm{fl} \mathrm{oz}(\mathrm{F} 10.5 .1+4-6 \mathrm{~d})\end{array}$ & 0.1 & 1.8 & 94.1 & $54.9 \mathrm{~b}$ & 1.8 \\
\hline $\begin{array}{l}\text { 8. Miravis ACE SE } 13.7 \mathrm{fl} \mathrm{oz}(\mathrm{F} 10.5 .1) \\
\text { Caramba 0.75 SL } 13.5 \mathrm{fl} \mathrm{oz}(\mathrm{F} 10.5 .1+4-6 \mathrm{~d})\end{array}$ & 0.0 & 0.5 & 105.7 & $56.2 \mathrm{a}$ & 1.8 \\
\hline $\begin{array}{ll}\text { 9. } & \text { Miravis ACE SE } 13.7 \mathrm{fl} \mathrm{oz}(\mathrm{F} 10.5 .1) \\
& \text { Tebuconazole 3.6 F } 4 \mathrm{fl} \mathrm{oz}(\mathrm{F} 10.5 .1+4-6 \mathrm{~d})\end{array}$ & 0.1 & 1.3 & 92.4 & $55.9 \mathrm{a}$ & 1.5 \\
\hline 10. Prosaro 421 SC $6.5 \mathrm{fl} \mathrm{oz} \mathrm{(F10.3)}$ & 0.0 & 0.4 & 93.2 & $54.7 \mathrm{~b}$ & 1.8 \\
\hline 11. Prosaro 421 SC $6.5 \mathrm{fl} \mathrm{oz}(\mathrm{F} 10.5 .1+4-6 \mathrm{~d})$ & 0.1 & 0.9 & 86.0 & $54.6 \mathrm{~b}$ & 1.8 \\
\hline 12. Caramba $0.75 \mathrm{SL} 13.5 \mathrm{fl} \mathrm{oz}(\mathrm{F} 10.3)$ & 0.0 & 0.3 & 86.2 & $54.4 \mathrm{~b}$ & 2.5 \\
\hline 13. Caramba 0.75 SL $13.5 \mathrm{fl} \mathrm{oz}(\mathrm{F} 10.5 .1+4-6 \mathrm{~d})$ & 0.1 & 0.7 & 91.5 & $54.8 \mathrm{~b}$ & 1.8 \\
\hline$P(\mathrm{~F})$ & 0.26 & 0.29 & 0.32 & $<0.0001$ & 0.70 \\
\hline LSD & N.S. & N.S. & N.S. & 0.88 & N.S. \\
\hline
\end{tabular}

z Fungicide sprays were applied at Feekes 3 on 21 Apr, Feekes 10.5.1 (flowering) on 28 Apr, and Feekes 10.5.1 + 4-6d on 4 May. Fusarium graminearum conidia $(\sim 50,000 / \mathrm{ml})$ applied on 29 Apr to all plots 24 hours following the Feekes 10.5.1 fungicide treatment. All fungicide treatments applied with Induce $0.125 \% \mathrm{v} / \mathrm{v}$.

y Percent of grain heads with signs and symptoms of Fusarium head blight.

$x$ Percent of spikelets with signs and symptoms of Fusarium head blight.

w Yields are weight of wheat with $13.5 \%$ moisture. One bushel equals $60 \mathrm{lbs}$. Wheat was harvested on 22 Jun.

$\checkmark$ Percent Fusarium damaged kernels. FDK rating scale: $0=$ no damage, $100=100 \%$ Fusarium damaged kernels in scabby wheat; FDK scale by Engle, De Wolf \& Lipps, Ohio State.

Means in a column followed by the same letter(s) are not significantly different according to Fisher's Protected LSD ( $P=0.05)$. Arcsine transformation of percentage data was made in analysis to determine statistical significance. 
TEST ID: WHTFOLFUN120

PURPOSE: To compare fungicide treatments for foliar disease control and impact on yield in wheat

LOCATION: Tidewater AREC Duke Farm, Holland Rd., Suffolk, VA

CROP INFORMATION:

\begin{tabular}{|l|l|}
\hline Field & $45 \mathrm{~A}$ \\
\hline Crop history & 2019 soy/corn, 2018 wheat/soy, 2017 soy/sorghum \\
\hline Planting date & 8 Dec 2019 \\
\hline Variety & DynaGrow 9811 \\
\hline Seeding rate & 30 seed/ft \\
\hline Plot length & $30^{\prime}$ \\
\hline Number of rows & 8 \\
\hline Row spacing & $7.5^{\prime \prime}$ \\
\hline Alleys (length between blocks) & $8^{\prime}$ \\
\hline Harvest date & 24 Jun \\
\hline
\end{tabular}

EXPERIMENTAL DESIGN: Randomized complete block with four replicates

TREATMENT APPLICATION:

\begin{tabular}{|l|l|}
\hline Equipment & Lee Spider sprayer \\
\hline Pressure (psi) & $38 \mathrm{psi}$ \\
\hline Nozzle type & Twinjet 8002VS \\
\hline Volume (gal/A) & 19.88 \\
\hline Surfactant & NIS $0.25 \% \mathrm{v} / \mathrm{v}$ \\
\hline
\end{tabular}

TREATMENTS:

\begin{tabular}{|l|l|l|l|l|}
\hline Trt \# & Fungicide and formulation & Rate, fl oz/A & App. timing & App. date \\
\hline 1 & Untreated & -- & -- & \\
\hline 2 & TopGuard & 7 & Feekes 9 & $16 \mathrm{Apr}$ \\
\hline 3 & TopGuard EQ & 5 & Feekes 9 & $16 \mathrm{Apr}$ \\
\hline 4 & TopGuard & 5 & Feekes 3-6 & 4 Mar \\
\hline 5 & Tilt 3.6 EC & 4 & Feekes 3-6 & 4 Mar \\
\hline 6 & TopGuard EQ & 3 & Feekes 3-6 & 4 Mar \\
\hline 7 & TopGuard & 5 & Feekes 3-6 & 4 Mar \\
& Miravis ACE & 13.7 & Feekes 10.5.1 & 4 May \\
\hline 8 & Tilt 3.6 EC & 4 & Feekes 3-6 & 4 Mar \\
& Miravis ACE & 13.7 & Feekes 10.5.1 & May \\
\hline 9 & TopGuard EQ & 3 & Feekes 3-6 & 4 Mar \\
& Miravis ACE & 13.7 & Feekes 10.5.1 & 4 May \\
\hline 10 & Miravis ACE & 13.7 & Feekes 10.5.1 & \\
\hline
\end{tabular}

SOIL TYPE: Lynchburg fine sandy loam

SOIL FERTILITY REPORT (Jan 2020):

\begin{tabular}{|l|c|c|c|c|c|c|c|c|c|}
\hline $\mathbf{p H}$ & $\mathbf{P}(\mathbf{l b} / \mathbf{A})$ & $\mathbf{K}(\mathbf{l b} / \mathbf{A})$ & $\mathbf{C a}(\mathbf{l b} / \mathbf{A})$ & $\mathbf{M g}(\mathbf{l b} / \mathbf{A})$ & $\mathbf{Z n}(\mathbf{p p m})$ & $\mathbf{M n}(\mathbf{p p m})$ & $\mathbf{C u}(\mathbf{p p m})$ & $\mathbf{F e}(\mathbf{p p m})$ & $\mathbf{B}(\mathbf{p p m})$ \\
\hline 6.0 & 50 & 165 & 621 & 96 & 0.4 & 2.8 & 0.3 & 32.4 & 0.1 \\
\hline
\end{tabular}


MAINTENANCE CHEMICAL PROGRAMS:

\begin{tabular}{|l|l|}
\hline Fertilizer & Standard \\
\hline Herbicides & Standard \\
\hline Insecticides & Standard \\
\hline Fungicides & None except treatments \\
\hline Nematicides & None \\
\hline
\end{tabular}

MAINTENANCE CHEMICAL APPLICATIONS:

\begin{tabular}{|l|l|l|l|}
\hline Date & Type and target & Product and formulation & Rate/A \\
\hline $1-30-20$ & Fertility & $9-18-31$ & $322 \mathrm{lbs}$ \\
\hline $2-4-20$ & Fertility & $24-0-0-3$ & 60 units \\
\hline $3-14-20$ & Fertility & $24-0-0-3$ & 60 units \\
\hline $2-4-20$ & Herbicide & Quelex & $0.75 \mathrm{fl} \mathrm{oz}$ \\
\hline
\end{tabular}

Table 1. Effect of fungicide treatment on disease incidence in wheat (WHTFOLFUN120, Suffolk, VA 2020).

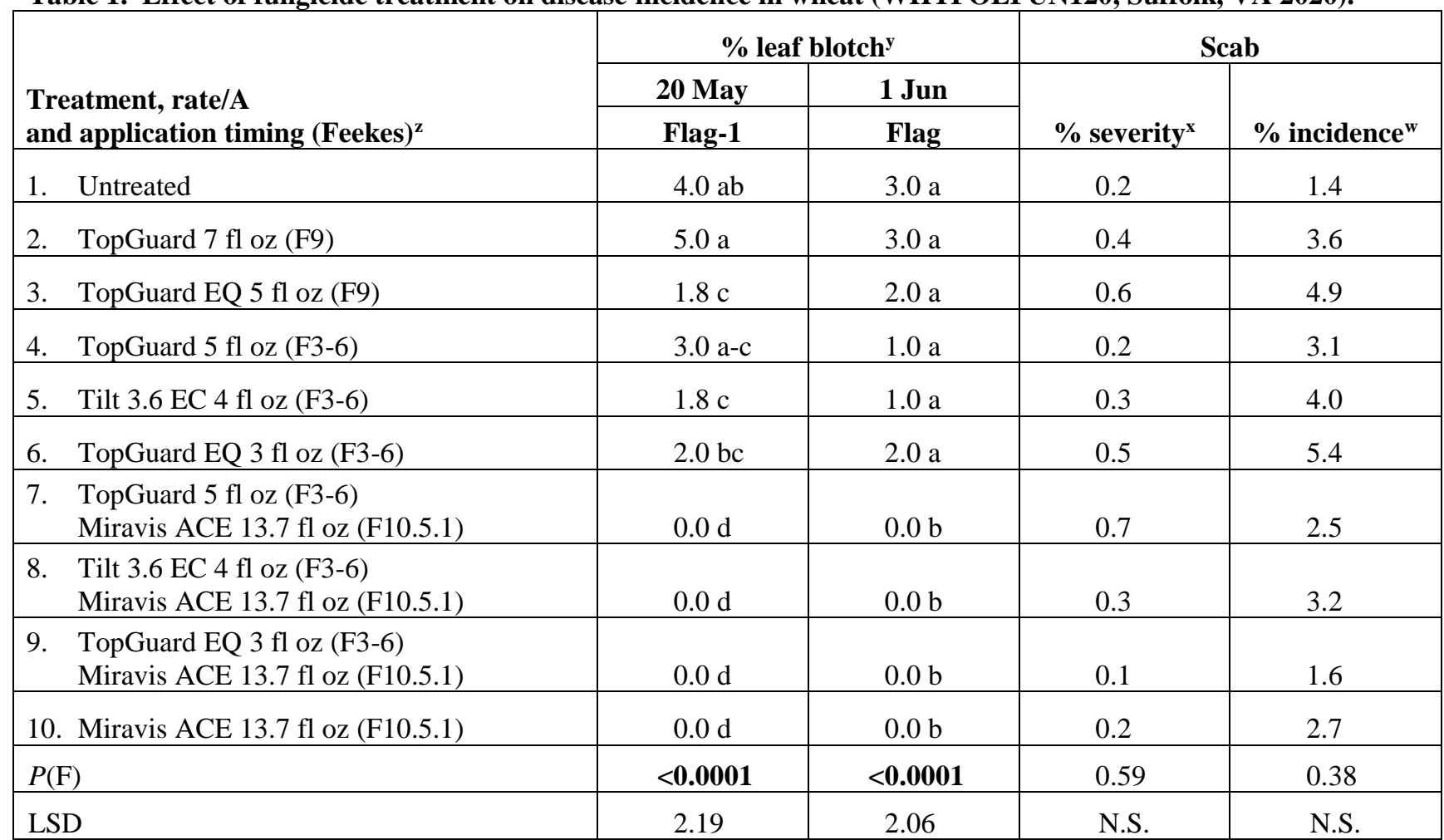

z Fungicide sprays were applied at Feekes 3-6 on 4 Mar, Feekes 9 on 16 Apr, and Feekes 10.5.1 on 4 May. All fungicide treatments applied with Induce $0.25 \% \mathrm{v} / \mathrm{v}$.

y Percent of leaf area with signs and symptoms of Stagnospora nodorum blotch. Plots were also evaluated for symptoms of powdery mildew and rust; none were observed.

$x$ Percent of grain heads with signs and symptoms of Fusarium head blight.

${ }^{w}$ Percent of spikelets with signs and symptoms of Fusarium head blight. Means in a column followed by the same letter(s) are not significantly different according to Fisher's Protected LSD ( $P=0.05)$. Arcsine transformation of percentage data was made in analysis to determine statistical significance. 
Table 2. Effect of fungicide treatment on disease severity and yield in wheat (WHTFOLFUN120, Suffolk, VA 2020).

\begin{tabular}{|l|c|c|c|}
\hline $\begin{array}{l}\text { Treatment, rate/A } \\
\text { and application timing (Feekes) }\end{array}$ & $\begin{array}{c}\text { Yield } \\
\text { (bu/A) }\end{array}$ & $\begin{array}{c}\text { Test weight } \\
\text { (lb/bu) }\end{array}$ & ${\text { \% } \text { FDK }^{\mathbf{x}}}^{\mathbf{y}}$ \\
\hline 1. Untreated & 65.2 & $54.1 \mathrm{bc}$ & $4.3 \mathrm{a}$ \\
\hline 2. TopGuard 7 fl oz (F9) & 77.3 & $54.4 \mathrm{bc}$ & $3.3 \mathrm{ab}$ \\
\hline 3. TopGuard EQ 5 fl oz (F9) & 73.7 & $54.5 \mathrm{~b}$ & $4.5 \mathrm{a}$ \\
\hline 4. TopGuard 5 fl oz (F3-6) & 69.8 & $53.7 \mathrm{c}$ & $2.3 \mathrm{~b}$ \\
\hline 5. Tilt 3.6 EC 5 fl oz (F3-6) & 69.3 & $54.0 \mathrm{bc}$ & $3.0 \mathrm{ab}$ \\
\hline 6. TopGuard EQ 3 fl oz (F3-6) & 69.0 & $53.8 \mathrm{bc}$ & $4.3 \mathrm{a}$ \\
\hline 7. TopGuard 5 fl oz (F3-6) & 65.0 & $55.3 \mathrm{a}$ & $1.5 \mathrm{~b}$ \\
\hline $\begin{array}{l}\text { 8iravis ACE 13.7 fl oz (F10.5.1) } \\
\quad \text { Miravis ACE 13.7 fl oz (F10.5.1) }\end{array}$ & 73.1 & $55.3 \mathrm{a}$ & $1.8 \mathrm{~b}$ \\
\hline $\begin{array}{l}\text { TopGuard EQ 3 fl oz (F3-6) } \\
\text { Miravis ACE 13.7 fl oz (F10.5.1) }\end{array}$ & 73.8 & $55.5 \mathrm{a}$ & $2.8 \mathrm{ab}$ \\
\hline 10. Miravis ACE 13.7 fl oz (F10.5.1) & 68.8 & $55.5 \mathrm{a}$ & $2.0 \mathrm{~b}$ \\
\hline P(F) & 0.32 & $<\mathbf{0 . 0 0 0 1}$ & $\mathbf{0 . 0 0 9}$ \\
\hline LSD & N.S. & 0.71 & 1.78 \\
\hline
\end{tabular}

z Fungicide sprays were applied at Feekes 3-6 on 4 Mar, Feekes 9 on 16 Apr, and Feekes 10.5.1 on 4 May. All fungicide treatments applied with Induce $0.25 \% \mathrm{v} / \mathrm{v}$.

y Yields are weight of wheat with $13.5 \%$ moisture. One bushel equals $60 \mathrm{lbs}$. Wheat was harvested on $24 \mathrm{Jun}$.

$\mathrm{x}$ Percent Fusarium damaged kernels. FDK rating scale: $0=$ no damage, $100=100 \%$ Fusarium damaged kernels in scabby wheat; FDK scale by Engle, De Wolf \& Lipps, Ohio State.

Means in a column followed by the same letter(s) are not significantly different according to Fisher's Protected LSD $(P=0.05)$. 
TEST ID: WHTFOLFUN220

PURPOSE: To compare fungicide treatments for foliar disease control and impact on yield in wheat

LOCATION: Tidewater Research Farm, Hare Rd., Suffolk, VA

CROP INFORMATION:

\begin{tabular}{|l|l|}
\hline Field & 28 \\
\hline Crop history & 2019 peanut, 2018 wheat/soy, 2017 peanut \\
\hline Planting date & 21 Nov 2019 \\
\hline Variety & Shirley \\
\hline Seeding rate & 30 seed/ft \\
\hline Plot length/width & $30^{\prime} \times 5^{\prime}$ (treat \& harvest), 30'x 12' total \\
\hline Number of rows & 8 \\
\hline Row spacing & $7.5^{\prime \prime}$ \\
\hline Alleys (length between blocks) & $8^{\prime}$ \\
\hline Harvest date & 24 Jun \\
\hline
\end{tabular}

EXPERIMENTAL DESIGN: Randomized complete block with four replicates

INOCULUM: Fusarium graminearum conidia ( 50,000/ml) applied 24 hours (29 Apr) following the Feekes 10.5.1 fungicide treatment with Lee Spider Sprayer; $1 \mathrm{~L}$ inoculum to $11 \mathrm{~L} \mathrm{H}_{2} \mathrm{O}$

TREATMENT APPLICATION:

\begin{tabular}{|l|l|}
\hline Equipment & Lee Spider sprayer \\
\hline Pressure (psi) & $38 \mathrm{psi}$ \\
\hline Nozzle type & Twinjet $8002 \mathrm{VS}$ \\
\hline Volume (gal/A) & 19.88 \\
\hline Surfactant & NIS $0.25 \% \mathrm{v} / \mathrm{v}$ \\
\hline
\end{tabular}

TREATMENTS:

\begin{tabular}{|l|l|l|l|l|}
\hline Trt \# & Fungicide and formulation & Rate, fl oz/A & App. timing & App. date \\
\hline 1 & Untreated & -- & -- & \\
\hline 2 & Folicur 3.6 F & 4 & Feekes 9 & $11 \mathrm{Apr}$ \\
\hline 3 & Tilt 3.6 EC & 4 & Feekes 9 & $11 \mathrm{Apr}$ \\
\hline 4 & Aproach Prima 2.34 SC & 6.8 & Feekes 9 & $11 \mathrm{Apr}$ \\
\hline 5 & Priaxor 4.17 SC & 4 & Feekes 9 & $11 \mathrm{Apr}$ \\
\hline 6 & Quilt Xcel 2.2 SE & 10.5 & Feekes 9 & $11 \mathrm{Apr}$ \\
\hline 7 & Stratego YLD & 4 & Feekes 9 & $11 \mathrm{Apr}$ \\
\hline 8 & Priaxor 4.17 SC & 4 & & \\
& + Tilt 3.6 EC & 4 & Feekes 9 & $11 \mathrm{Apr}$ \\
\hline 9 & Caramba 0.75 SL & 13.5 & Feekes 10.5.1 & $28 \mathrm{Apr}$ \\
\hline 10 & Folicur 3.6 F & 4 & Feekes 10.5.1 & $28 \mathrm{Apr}$ \\
\hline 11 & Proline 480 SC & 5.7 & Feekes 10.5.1 & $28 \mathrm{Apr}$ \\
\hline 12 & Prosaro 421 SC & 6.5 & Feekes 10.5.1 & $28 \mathrm{Apr}$ \\
\hline 13 & Tilt 3.6 EC & 4 & Feekes 10.5.1 & $28 \mathrm{Apr}$ \\
\hline 14 & Miravis ACE SE & 13.7 & Feekes 10.5.1 & $28 \mathrm{Apr}$ \\
\hline
\end{tabular}


SOIL TYPE: Kenansville loamy sand

SOIL FERTILITY REPORT (Oct 2019):

\begin{tabular}{|c|c|c|c|c|c|c|c|c|c|}
\hline $\mathbf{p H}$ & $\mathbf{P}(\mathbf{l b} / \mathbf{A})$ & $\mathbf{K}(\mathbf{l b} / \mathbf{A})$ & $\mathbf{C a}(\mathbf{l b} / \mathbf{A})$ & $\mathbf{M g}(\mathbf{l b} / \mathbf{A})$ & $\mathbf{Z n}(\mathbf{p p m})$ & $\mathbf{M n}(\mathbf{p p m})$ & $\mathbf{C u}(\mathbf{p p m})$ & $\mathbf{F e}(\mathbf{p p m})$ & $\mathbf{B}(\mathbf{p p m})$ \\
\hline 5.9 & 27 & 190 & 800 & 77 & 0.3 & 4.8 & 0.2 & 10.7 & 0.2 \\
\hline
\end{tabular}

MAINTENANCE CHEMICAL PROGRAMS:

\begin{tabular}{|l|l|}
\hline Fertilizer & Standard \\
\hline Herbicides & Standard \\
\hline Insecticides & Standard \\
\hline Fungicides & None except treatments \\
\hline Nematicides & None \\
\hline
\end{tabular}

MAINTENANCE CHEMICAL APPLICATIONS:

\begin{tabular}{|l|l|l|l|}
\hline Date & Type and target & Product and formulation & Rate/A \\
\hline $1-30-20$ & Fertility & $9-18-31$ & $322 \mathrm{lbs}$ \\
\hline $2-4-20$ & Fertility & $24-0-0-3$ & 60 units \\
\hline $3-14-20$ & Fertility & $24-0-0-3$ & 60 units \\
\hline $2-4-20$ & Herbicide & Quelex & $0.75 \mathrm{fl} \mathrm{oz}$ \\
\hline
\end{tabular}


Table 1. Effect of fungicide treatment on disease incidence in wheat (WHTFOLFUN220, Suffolk, VA 2020).

\begin{tabular}{|c|c|c|c|c|c|}
\hline \multirow{3}{*}{$\begin{array}{l}\text { Treatment, rate/A } \\
\text { and application timing }(\text { Feekes })^{\mathrm{z}}\end{array}$} & \multicolumn{5}{|c|}{$\%$ leaf blotch ${ }^{y}$} \\
\hline & \multirow{2}{*}{$\begin{array}{l}5 \text { May } \\
\text { Flag-2 }\end{array}$} & \multirow{2}{*}{$\begin{array}{l}14 \text { May } \\
\text { Flag-1 }\end{array}$} & \multirow{2}{*}{$\begin{array}{c}20 \text { May } \\
\text { Flag-1 }\end{array}$} & \multicolumn{2}{|c|}{26 May } \\
\hline & & & & Flag & Flag-1 \\
\hline 1. Untreated & $3.0 \mathrm{a}-\mathrm{c}$ & $5.0 \mathrm{bc}$ & $12.5 \mathrm{~b}$ & 1.8 & $11.3 \mathrm{bc}$ \\
\hline 2. Folicur 3.6 F 4 fl oz (F9) & $5.0 \mathrm{ab}$ & $6.5 \mathrm{bc}$ & $6.3 \mathrm{~cd}$ & 1.5 & $12.5 \mathrm{a}-\mathrm{c}$ \\
\hline 3. Tilt $3.6 \mathrm{EC} 4 \mathrm{fl} \mathrm{oz}(\mathrm{F} 9)$ & $0.5 \mathrm{~d}$ & $1.0 \mathrm{~d}$ & $4.0 \mathrm{de}$ & 0.5 & $8.8 \mathrm{bc}$ \\
\hline 4. Aproach Prima 2.34 SC $6.8 \mathrm{fl} \mathrm{oz} \mathrm{(F9)}$ & $2.0 \mathrm{~b}-\mathrm{d}$ & $4.3 \mathrm{~cd}$ & $10.0 \mathrm{bc}$ & 0.8 & $16.3 \mathrm{ab}$ \\
\hline 5. Priaxor 4.17 SC $4 \mathrm{fl} \mathrm{oz} \mathrm{(F9)}$ & $7.5 \mathrm{a}$ & $11.3 \mathrm{ab}$ & $22.5 \mathrm{a}$ & 2.0 & $21.3 \mathrm{a}$ \\
\hline 6. Quilt Xcel 2.2 SE $10.5 \mathrm{fl} \mathrm{oz} \mathrm{(F9)}$ & $2.0 \mathrm{~b}-\mathrm{d}$ & $1.0 \mathrm{~d}$ & $3.0 \mathrm{~d}-\mathrm{f}$ & 0.5 & $12.5 \mathrm{a}-\mathrm{c}$ \\
\hline 7. Stratego YLD $4 \mathrm{fl} \mathrm{oz} \mathrm{(F9)}$ & $1.5 \mathrm{~cd}$ & $6.8 \mathrm{~b}-\mathrm{d}$ & $8.8 \mathrm{bc}$ & 2.0 & $13.8 \mathrm{a}-\mathrm{c}$ \\
\hline $\begin{array}{ll}\text { 8. } & \text { Priaxor 4.17 SC } 4 \text { fl oz } \\
& + \text { Tilt 3.6 EC } 4 \text { fl oz (F9) } \\
\end{array}$ & $3.0 \mathrm{a}-\mathrm{c}$ & $4.0 \mathrm{~cd}$ & $3.0 \mathrm{~d}-\mathrm{f}$ & 1.8 & $8.8 \mathrm{~b}-\mathrm{d}$ \\
\hline 9. Caramba 0.75 SL $13.5 \mathrm{fl} \mathrm{oz}(\mathrm{F} 10.5 .1)$ & $1.8 \mathrm{~b}-\mathrm{d}$ & $4.3 \mathrm{~cd}$ & $0.8 \mathrm{f}$ & 0.0 & $3.8 \mathrm{~d}-\mathrm{f}$ \\
\hline 10. Folicur 3.6 F $4 \mathrm{fl} \mathrm{oz} \mathrm{(F10.5.1)}$ & $4.0 \mathrm{ab}$ & $4.3 \mathrm{~cd}$ & $5.0 \mathrm{~cd}$ & 0.3 & $7.5 \mathrm{c}-\mathrm{e}$ \\
\hline 11. Proline 480 SC $5.7 \mathrm{fl} \mathrm{oz}(\mathrm{F} 10.5 .1)$ & $4.0 \mathrm{ab}$ & $13.8 \mathrm{a}$ & $15.0 \mathrm{~b}$ & 1.5 & $15.0 \mathrm{a}-\mathrm{c}$ \\
\hline 12. Prosaro 421 SC $6.5 \mathrm{fl} \mathrm{oz}(\mathrm{F} 10.5 .1)$ & $5.5 \mathrm{ab}$ & $5.8 \mathrm{~cd}$ & $5.3 \mathrm{~cd}$ & 0.3 & $11.3 \mathrm{bc}$ \\
\hline 13. Tilt $3.6 \mathrm{EC} 4 \mathrm{fl} \mathrm{oz}$ (F10.5.1) & $2.0 \mathrm{~b}-\mathrm{d}$ & $2.0 \mathrm{~cd}$ & $1.8 \mathrm{ef}$ & 0.0 & $3.0 \mathrm{ef}$ \\
\hline 14. Miravis ACE SE $13.7 \mathrm{fl} \mathrm{oz} \mathrm{(F10.5.1)}$ & $5.0 \mathrm{ab}$ & $6.5 \mathrm{bc}$ & $3.0 \mathrm{~d}-\mathrm{f}$ & 0.0 & $0.8 \mathrm{f}$ \\
\hline$P(\mathrm{~F})$ & 0.02 & 0.003 & $<0.0001$ & 0.054 & $<0.0001$ \\
\hline LSD & 3.77 & 6.38 & 5.50 & N.S. & 7.76 \\
\hline
\end{tabular}

$\mathrm{z}$ Fungicide sprays were applied at Feekes 9 on 11 Apr and Feekes 10.5.1 (flowering) on 28 Apr. Fusarium graminearum conidia $(\sim 50,000 / \mathrm{ml})$ applied 24 hours (29 Apr) to all plots following the Feekes 10.5.1 fungicide treatment (29 Apr). All fungicide treatments were applied with Induce $0.25 \% \mathrm{v} / \mathrm{v}$.

y Percent of leaf area with signs and symptoms of Stagnospora nodorum blotch. Plots were also evaluated for symptoms of powdery mildew; none were observed.

Means in a column or group followed by the same letter(s) are not significantly different according to Fisher's Protected LSD $(P=0.05)$. Arcsine transformation of percentage data was made in analysis to determine statistical significance. 
Table 2. Effect of fungicide treatment on disease severity in wheat (WHTFOLFUN220, Suffolk, VA 2020).

\begin{tabular}{|c|c|c|c|c|c|c|c|}
\hline \multirow{4}{*}{$\begin{array}{l}\text { Treatment, rate/A } \\
\text { and application timing (Feekes) }{ }^{\mathrm{z}}\end{array}$} & \multicolumn{7}{|c|}{$\%$ foliar disease } \\
\hline & \multicolumn{3}{|c|}{ Leaf rust ${ }^{y}$} & \multicolumn{4}{|c|}{ Stripe rust ${ }^{\mathrm{x}}$} \\
\hline & \multirow{2}{*}{$\begin{array}{l}14 \text { May } \\
\text { Flag-1 }\end{array}$} & \multicolumn{2}{|c|}{26 May } & \multirow{2}{*}{$\begin{array}{c}14 \text { May } \\
\text { Flag }\end{array}$} & \multirow{2}{*}{$\begin{array}{c}20 \text { May } \\
\text { Flag }\end{array}$} & \multicolumn{2}{|c|}{26 May } \\
\hline & & Flag & Flag-1 & & & Flag & Flag-1 \\
\hline 1. Untreated & 3.0 & 0.8 & 6.3 & 5.3 & 3.8 & $5.5 \mathrm{a}$ & $11.3 \mathrm{a}$ \\
\hline 2. Folicur $3.6 \mathrm{~F} 4 \mathrm{fl} \mathrm{oz}(\mathrm{F} 9)$ & 6.5 & 0.0 & 5.0 & 0.0 & 0.0 & $0.3 \mathrm{~b}$ & $0.3 \mathrm{~b}$ \\
\hline 3. Tilt 3.6 EC $4 \mathrm{fl} \mathrm{oz}(\mathrm{F} 9)$ & 4.0 & 0.3 & 6.3 & 0.0 & 0.0 & $0.0 \mathrm{~b}$ & $0.0 \mathrm{~b}$ \\
\hline 4. Aproach Prima 2.34 SC $6.8 \mathrm{fl} \mathrm{oz} \mathrm{(F9)}$ & 4.0 & 0.0 & 5.0 & 0.0 & 0.0 & $0.0 \mathrm{~b}$ & $0.0 \mathrm{~b}$ \\
\hline 5. Priaxor $4.17 \mathrm{SC} 4 \mathrm{fl} \mathrm{oz}(\mathrm{F} 9)$ & 4.0 & 0.5 & 8.8 & 1.3 & 0.0 & $0.0 \mathrm{~b}$ & $0.0 \mathrm{~b}$ \\
\hline 6. Quilt Xcel 2.2 SE $10.5 \mathrm{fl} \mathrm{oz} \mathrm{(F9)}$ & 1.0 & 0.3 & 4.0 & 0.0 & 0.0 & $0.0 \mathrm{~b}$ & $0.0 \mathrm{~b}$ \\
\hline 7. Stratego YLD $4 \mathrm{fl} \mathrm{oz} \mathrm{(F9)}$ & 5.0 & 1.5 & 6.3 & 0.0 & 0.3 & $0.0 \mathrm{~b}$ & $0.0 \mathrm{~b}$ \\
\hline $\begin{array}{l}\text { 8. Priaxor 4.17 SC } 4 \text { fl oz } \\
+ \text { Tilt 3.6 EC } 4 \text { fl oz (F9) }\end{array}$ & 3.0 & 1.8 & 7.5 & 0.0 & 0.0 & $0.0 \mathrm{~b}$ & $0.0 \mathrm{~b}$ \\
\hline 9. Caramba 0.75 SL $13.5 \mathrm{fl} \mathrm{oz} \mathrm{(F10.5.1)}$ & 2.8 & 0.0 & 6.3 & 0.0 & 0.0 & $0.0 \mathrm{~b}$ & $0.0 \mathrm{~b}$ \\
\hline 10. Folicur $3.6 \mathrm{~F} 4 \mathrm{fl} \mathrm{oz}(\mathrm{F} 10.5 .1)$ & 2.8 & 0.3 & 6.3 & 0.0 & 0.0 & $0.0 \mathrm{~b}$ & $0.0 \mathrm{~b}$ \\
\hline 11. Proline $480 \mathrm{SC} 5.7 \mathrm{fl} \mathrm{oz}(\mathrm{F} 10.5 .1)$ & 4.0 & 0.0 & 5.0 & 0.0 & 0.0 & $0.0 \mathrm{~b}$ & $0.0 \mathrm{~b}$ \\
\hline 12. Prosaro $421 \mathrm{SC} 6.5 \mathrm{fl} \mathrm{oz}(\mathrm{F} 10.5 .1)$ & 2.8 & 0.0 & 5.0 & 0.0 & 0.0 & $0.0 \mathrm{~b}$ & $0.0 \mathrm{~b}$ \\
\hline 13. Tilt $3.6 \mathrm{EC} 4 \mathrm{fl} \mathrm{oz}(\mathrm{F} 10.5 .1)$ & 7.5 & 0.0 & 5.0 & 0.0 & 0.0 & $0.0 \mathrm{~b}$ & $0.0 \mathrm{~b}$ \\
\hline 14. Miravis ACE SE $13.7 \mathrm{fl} \mathrm{oz}$ (F10.5.1) & 2.0 & 1.3 & 7.5 & 0.0 & 0.0 & $0.0 \mathrm{~b}$ & $0.0 \mathrm{~b}$ \\
\hline$P(\mathrm{~F})$ & 0.25 & 0.13 & 0.74 & 0.15 & 0.47 & 0.01 & $<0.0001$ \\
\hline LSD & N.S. & N.S. & N.S. & N.S. & N.S. & 3.69 & 5.02 \\
\hline
\end{tabular}

z Fungicide sprays were applied at Feekes 9 on 11 Apr and Feekes 10.5.1 (flowering) on 28 Apr. Fusarium graminearum conidia $(\sim 50,000 / \mathrm{ml})$ applied to all plots 24 hours following the Feekes 10.5.1 fungicide treatment (29 Apr). All fungicide treatments were applied with Induce $0.25 \% \mathrm{v} / \mathrm{v}$.

y Percent of leaf area with signs and symptoms of Puccinia recondite f. sp. tritici.

$x$ Percent of leaf area with signs and symptoms of Puccinia striiformis f. sp. tritici.

Means in a column or group followed by the same letter(s) are not significantly different according to Fisher's Protected LSD $(P=0.05)$. Arcsine transformation of percentage data was made in analysis to determine statistical significance. 
Table 3. Effect of fungicide treatment on disease severity and yield in wheat (WHTFOLFUN220, Suffolk, VA 2020).

\begin{tabular}{|c|c|c|c|c|c|}
\hline \multirow[b]{2}{*}{$\begin{array}{l}\text { Treatment, rate/A } \\
\text { and application timing }(\text { Feekes })^{\mathrm{z}}\end{array}$} & \multicolumn{2}{|c|}{$\begin{array}{c}\text { Scab } \\
21 \text { May } \\
\end{array}$} & \multirow[b]{2}{*}{$\begin{array}{l}\text { Yield }^{w} \\
(\text { bu/A) }\end{array}$} & \multirow[b]{2}{*}{$\begin{array}{c}\text { Test weight } \\
(\mathrm{lb} / \mathrm{bu})\end{array}$} & \multirow[b]{2}{*}{$\%$ FDK $^{\mathbf{r}}$} \\
\hline & $\begin{array}{c}\% \\
\text { severity }^{\mathrm{y}} \\
\end{array}$ & $\begin{array}{c}\% \\
\text { incidence }^{x} \\
\end{array}$ & & & \\
\hline 1. Untreated & 0.1 & $0.8 \mathrm{bc}$ & 89.2 & $52.6 \mathrm{~d}$ & 3.3 \\
\hline 2. Folicur $3.6 \mathrm{~F} 4 \mathrm{fl} \mathrm{oz}(\mathrm{F9})$ & 0.1 & $0.3 \mathrm{c}$ & 94.7 & $52.8 \mathrm{~d}$ & 2.0 \\
\hline 3. Tilt $3.6 \mathrm{EC} 4 \mathrm{fl} \mathrm{oz}(\mathrm{F} 9)$ & 0.1 & $0.9 \mathrm{bc}$ & 96.1 & $53.4 \mathrm{~b}-\mathrm{d}$ & 2.8 \\
\hline 4. Aproach Prima 2.34 SC $6.8 \mathrm{fl} \mathrm{oz} \mathrm{(F9)}$ & 0.1 & $1.4 \mathrm{a}-\mathrm{c}$ & 105.2 & $53.3 \mathrm{~b}-\mathrm{d}$ & 2.3 \\
\hline 5. Priaxor $4.17 \mathrm{SC} 4 \mathrm{fl} \mathrm{oz}(\mathrm{F} 9)$ & 0.0 & $0.3 \mathrm{c}$ & 103.0 & $53.4 \mathrm{~b}-\mathrm{d}$ & 2.5 \\
\hline 6. Quilt Xcel 2.2 SE $10.5 \mathrm{fl} \mathrm{oz} \mathrm{(F9)}$ & 0.1 & $0.6 \mathrm{bc}$ & 101.0 & $53.3 \mathrm{~b}-\mathrm{d}$ & 2.0 \\
\hline 7. Stratego YLD 4 fl oz (F9) & 0.1 & $0.3 \mathrm{c}$ & 85.2 & $52.9 \mathrm{~cd}$ & 2.5 \\
\hline $\begin{array}{l}\text { 8. Priaxor } 4.17 \mathrm{SC} 4 \mathrm{fl} \mathrm{oz} \\
+ \text { Tilt } 3.6 \mathrm{EC} 4 \mathrm{fl} \mathrm{oz}(\mathrm{F} 9)\end{array}$ & 0.1 & $1.1 \mathrm{bc}$ & 104.8 & $52.9 \mathrm{~cd}$ & 2.0 \\
\hline 9. Caramba 0.75 SL $13.5 \mathrm{fl} \mathrm{oz} \mathrm{(F10.5.1)}$ & 0.1 & $1.4 \mathrm{a}-\mathrm{c}$ & 101.7 & $53.6 \mathrm{~b}-\mathrm{d}$ & 2.3 \\
\hline 10. Folicur 3.6 F 4 fl oz (F10.5.1) & 0.0 & $0.0 \mathrm{c}$ & 101.1 & $53.4 \mathrm{~b}-\mathrm{d}$ & 2.8 \\
\hline 11. Proline 480 SC 5.7 fl oz (F10.5.1) & 0.3 & $3.6 \mathrm{a}$ & 105.9 & $53.1 \mathrm{~b}-\mathrm{d}$ & 2.5 \\
\hline 12. Prosaro $421 \mathrm{SC} 6.5 \mathrm{fl} \mathrm{oz}(\mathrm{F} 10.5 .1)$ & 0.1 & $1.1 \mathrm{a}-\mathrm{c}$ & 102.2 & $54.2 \mathrm{ab}$ & 2.8 \\
\hline 13. Tilt $3.6 \mathrm{EC} 4 \mathrm{fl} \mathrm{oz}(\mathrm{F} 10.5 .1)$ & 0.2 & $2.6 \mathrm{ab}$ & 106.3 & $55.0 \mathrm{a}$ & 2.0 \\
\hline 14. Miravis ACE SE $13.7 \mathrm{fl} \mathrm{oz}(\mathrm{F} 10.5 .1)$ & 0.0 & $0.0 \mathrm{c}$ & 99.7 & $54.1 \mathrm{a}-\mathrm{c}$ & 2.3 \\
\hline$P(\mathrm{~F})$ & 0.14 & 0.04 & 0.17 & $\mathbf{0 . 0 3}$ & 0.78 \\
\hline LSD & N.S. & 2.03 & N.S. & 1.22 & N.S. \\
\hline
\end{tabular}

z Fungicide sprays were applied at Feekes 9 on 11 Apr and Feekes 10.5.1 (flowering) on 28 Apr. Fusarium graminearum conidia $(\sim 50,000 / \mathrm{ml})$ applied to all plots 24 hours following the Feekes 10.5.1 fungicide treatment (29 Apr). All fungicide treatments were applied with Induce $0.25 \% \mathrm{v} / \mathrm{v}$.

y Percent of grain heads with signs and symptoms of Fusarium head blight.

$x$ Percent of spikelets with signs and symptoms of Fusarium head blight.

w Yields are weight of wheat with $13.5 \%$ moisture. One bushel equals $60 \mathrm{lbs}$. Wheat was harvested on 24 Jun.

$\checkmark$ Percent Fusarium damaged kernels. FDK rating scale: $0=$ no damage, $100=100 \%$ Fusarium damaged kernels in scabby wheat; FDK scale by Engle, De Wolf \& Lipps, Ohio State.

Means in a column followed by the same letter(s) are not significantly different according to Fisher's Protected LSD $(P=0.05)$. Arcsine transformation of percentage data was made in analysis to determine statistical significance. 
TEST ID: WHTFOLFUN320

PURPOSE: To compare fungicide treatments for foliar disease control and impact on yield in wheat

LOCATION: Tidewater Research Farm, Hare Rd., Suffolk, VA

CROP INFORMATION:

\begin{tabular}{|l|l|}
\hline Field & 28 \\
\hline Crop history & 2019 peanut, 2018 wheat/soy, 2017 peanut \\
\hline Planting date & 8 Dec 2019 \\
\hline Variety & DynaGrow 1198 \\
\hline Seeding rate & 30 seed/ft \\
\hline Plot length/width & $30^{\prime} \times 5^{\prime}$ (treat \& harvest), 30' x 12' total \\
\hline Number of rows & 8 \\
\hline Row spacing & $7.5^{\prime \prime}$ \\
\hline Alleys (length between blocks) & $8^{\prime}$ \\
\hline Harvest date & 24 Jun \\
\hline
\end{tabular}

EXPERIMENTAL DESIGN: Randomized complete block with four replicates

TREATMENT APPLICATION:

\begin{tabular}{|l|l|}
\hline Equipment & Lee Spider sprayer \\
\hline Pressure (psi) & $38 \mathrm{psi}$ \\
\hline Nozzle type & Twinjet 8002VS \\
\hline Volume (gal/A) & 19.88 \\
\hline Surfactant & NIS $0.25 \% \mathrm{v} / \mathrm{v}$ \\
\hline
\end{tabular}

TREATMENTS:

\begin{tabular}{|l|l|l|l|l|}
\hline Trt \# & Fungicide and formulation & Rate, fl oz/A & App. timing & App. date \\
\hline 1 & Untreated & -- & -- & \\
\hline 2 & Folicur 3.6 F & 4 & Feekes 9 & $11 \mathrm{Apr}$ \\
\hline 3 & Tilt 3.6 EC & 4 & Feekes 9 & $11 \mathrm{Apr}$ \\
\hline 4 & Aproach Prima 2.34 SC & 6.8 & Feekes 9 & $11 \mathrm{Apr}$ \\
\hline 5 & Priaxor 4.17 SC & 4 & Feekes 9 & $11 \mathrm{Apr}$ \\
\hline 6 & Quilt Xcel 2.2 SE & 10.5 & Feekes 9 & $11 \mathrm{Apr}$ \\
\hline 7 & Stratego YLD & 4 & Feekes 9 & $11 \mathrm{Apr}$ \\
\hline 8 & Priaxor 4.17 SC \\
+ Tilt 3.6 EC & 4 & Feekes 9 & $11 \mathrm{Apr}$ \\
\hline 9 & Caramba 0.75 SL & 4 & Feekes 10.5.1 & $21 \mathrm{Apr}$ \\
\hline 10 & Folicur 3.6 F & 43.5 & Feekes 10.5.1 & $21 \mathrm{Apr}$ \\
\hline 11 & Proline 480 SC & 5.7 & Feekes 10.5.1 & $21 \mathrm{Apr}$ \\
\hline 12 & Prosaro 421 SC & 6.5 & Feekes 10.5.1 & $21 \mathrm{Apr}$ \\
\hline 13 & Tilt 3.6 EC & 4 & Feekes 10.5.1 & $21 \mathrm{Apr}$ \\
\hline 14 & Miravis ACE SE & 13.7 & Feekes 10.5.1 & $21 \mathrm{Apr}$ \\
\hline
\end{tabular}


SOIL TYPE: Kenansville loamy sand

SOIL FERTILITY REPORT (Oct 2019):

\begin{tabular}{|c|c|c|c|c|c|c|c|c|c|}
\hline $\mathbf{p H}$ & $\mathbf{P}(\mathbf{l b} / \mathbf{A})$ & $\mathbf{K}(\mathbf{l b} / \mathbf{A})$ & $\mathbf{C a}(\mathbf{l b} / \mathbf{A})$ & $\mathbf{M g}(\mathbf{l b} / \mathbf{A})$ & $\mathbf{Z n}(\mathbf{p p m})$ & $\mathbf{M n}(\mathbf{p p m})$ & $\mathbf{C u}(\mathbf{p p m})$ & $\mathbf{F e}(\mathbf{p p m})$ & $\mathbf{B}(\mathbf{p p m})$ \\
\hline 5.9 & 27 & 190 & 800 & 77 & 0.3 & 4.8 & 0.2 & 10.7 & 0.2 \\
\hline
\end{tabular}

MAINTENANCE CHEMICAL PROGRAMS:

\begin{tabular}{|l|l|}
\hline Fertilizer & Standard \\
\hline Herbicides & Standard \\
\hline Insecticides & Standard \\
\hline Fungicides & None except treatments \\
\hline Nematicides & None \\
\hline
\end{tabular}

MAINTENANCE CHEMICAL APPLICATIONS:

\begin{tabular}{|l|l|l|l|}
\hline Date & Type and target & Product and formulation & Rate/A \\
\hline $1-30-20$ & Fertility & $9-18-31$ & $322 \mathrm{lbs}$ \\
\hline $2-4-20$ & Fertility & $24-0-0-3$ & 60 units \\
\hline $3-14-20$ & Fertility & $24-0-0-3$ & 60 units \\
\hline $2-4-20$ & Herbicide & Quelex & $0.75 \mathrm{fl} \mathrm{oz}$ \\
\hline
\end{tabular}


Table 1. Effect of fungicide treatment on disease severity in wheat (WHTFOLFUN320, Suffolk, VA 2020).

\begin{tabular}{|c|c|c|c|c|c|c|}
\hline \multirow{4}{*}{$\begin{array}{l}\text { Treatment, rate/A } \\
\text { and application timing }\left(\text { Feekes) }{ }^{\mathrm{z}}\right.\end{array}$} & \multicolumn{6}{|c|}{ \% foliar disease } \\
\hline & \multicolumn{5}{|c|}{ Leaf blotch } & \multirow{3}{*}{$\begin{array}{c}\begin{array}{c}\text { Leaf } \\
\text { rust }^{\mathrm{x}}\end{array} \\
\text { 14 May } \\
\text { Flag 1 }\end{array}$} \\
\hline & \multirow{2}{*}{$\begin{array}{l}4 \text { May } \\
\text { Flag-1 }\end{array}$} & \multirow{2}{*}{$\begin{array}{l}4 \text { May } \\
\text { Flag-2 }\end{array}$} & \multirow{2}{*}{$\begin{array}{c}14 \text { May } \\
\text { Flag-1 }\end{array}$} & \multicolumn{2}{|c|}{20 May } & \\
\hline & & & & Flag & Flag-1 & \\
\hline 1. Untreated & $0.8 \mathrm{ab}$ & 7.5 & $8.8 \mathrm{a}$ & 1.8 & $11.5 \mathrm{a}$ & 1.8 \\
\hline 2. Folicur 3.6 F 4 fl oz (F9) & $0.3 \mathrm{bc}$ & 6.3 & $3.0 \mathrm{~cd}$ & 0.0 & $6.3 \mathrm{ab}$ & 2.0 \\
\hline 3. Tilt $3.6 \mathrm{EC} 4 \mathrm{fl} \mathrm{oz}(\mathrm{F} 9)$ & $0.0 \mathrm{c}$ & 4.0 & $2.0 \mathrm{~d}$ & 0.0 & $5.0 \mathrm{a}-\mathrm{c}$ & 2.0 \\
\hline 4. Aproach Prima 2.34 SC $6.8 \mathrm{fl} \mathrm{oz} \mathrm{(F9)}$ & $0.8 \mathrm{ab}$ & 4.0 & $2.8 \mathrm{~d}$ & 0.3 & $11.3 \mathrm{a}$ & 2.8 \\
\hline 5. Priaxor 4.17 SC $4 \mathrm{fl} \mathrm{oz}(\mathrm{F9})$ & $0.5 \mathrm{a}-\mathrm{c}$ & 4.3 & $5.0 \mathrm{a}-\mathrm{d}$ & 0.8 & $12.5 \mathrm{a}$ & 4.0 \\
\hline 6. Quilt Xcel 2.2 SE $10.5 \mathrm{fl} \mathrm{oz} \mathrm{(F9)}$ & $0.3 \mathrm{bc}$ & 4.0 & $3.0 \mathrm{~cd}$ & 0.0 & $6.3 \mathrm{ab}$ & 3.8 \\
\hline 7. Stratego YLD $4 \mathrm{fl} \mathrm{oz} \mathrm{(F9)}$ & $1.5 \mathrm{ab}$ & 6.3 & $7.5 \mathrm{ab}$ & 1.8 & $10.0 \mathrm{a}$ & 1.5 \\
\hline $\begin{array}{l}\text { 8. Priaxor 4.17 SC } 4 \mathrm{fl} \mathrm{oz} \\
+ \text { Tilt 3.6 EC } 4 \mathrm{fl} \mathrm{oz}(\mathrm{F} 9)\end{array}$ & $0.0 \mathrm{c}$ & 4.0 & $2.0 \mathrm{~d}$ & 0.0 & $3.0 \mathrm{~b}-\mathrm{c}$ & 1.8 \\
\hline 9. Caramba 0.75 SL $13.5 \mathrm{fl} \mathrm{oz} \mathrm{(F10.5.1)}$ & $0.0 \mathrm{c}$ & 2.0 & $2.0 \mathrm{~d}$ & 0.0 & $1.0 \mathrm{c}$ & 3.0 \\
\hline 10. Folicur 3.6 F $4 \mathrm{fl} \mathrm{oz}(\mathrm{F} 10.5 .1)$ & $0.3 \mathrm{bc}$ & 4.0 & $3.0 \mathrm{~cd}$ & 0.3 & $5.0 \mathrm{a}-\mathrm{c}$ & 5.0 \\
\hline 11. Proline 480 SC $5.7 \mathrm{fl} \mathrm{oz}(\mathrm{F} 10.5 .1)$ & $0.3 \mathrm{bc}$ & 4.0 & $4.0 \mathrm{~b}-\mathrm{d}$ & 0.3 & $6.3 \mathrm{ab}$ & 2.0 \\
\hline 12. Prosaro 421 SC $6.5 \mathrm{fl} \mathrm{oz}(\mathrm{F} 10.5 .1)$ & $0.3 \mathrm{bc}$ & 4.0 & $6.3 \mathrm{a}-\mathrm{c}$ & 0.3 & $7.5 \mathrm{ab}$ & 3.0 \\
\hline 13. Tilt $3.6 \mathrm{EC} 4 \mathrm{fl} \mathrm{oz}(\mathrm{F} 10.5 .1)$ & $1.8 \mathrm{a}$ & 6.5 & $6.5 \mathrm{a}-\mathrm{c}$ & 1.3 & $7.8 \mathrm{ab}$ & 3.8 \\
\hline 14. Miravis ACE SE $13.7 \mathrm{fl} \mathrm{oz}(\mathrm{F} 10.5 .1)$ & $0.0 \mathrm{c}$ & 3.0 & $4.3 \mathrm{~b}-\mathrm{d}$ & 0.3 & $6.8 \mathrm{a}-\mathrm{c}$ & 4.0 \\
\hline$P(\mathrm{~F})$ & 0.049 & 0.34 & $\mathbf{0 . 0 2}$ & 0.06 & 0.04 & 0.60 \\
\hline LSD & 1.32 & N.S. & 3.72 & N.S. & 7.21 & N.S. \\
\hline
\end{tabular}

z Fungicide sprays were applied at Feekes 9 on 11 Apr and Feekes 10.5.1 (flowering) on 28 Apr. All fungicide treatments were applied with Induce $0.25 \% \mathrm{v} / \mathrm{v}$.

y Percent of leaf area with signs and symptoms of Stagnospora nodorum blotch. Plots were also evaluated for symptoms of powdery mildew; none were observed.

$x$ Percent of leaf area with signs and symptoms of Puccinia recondite f. sp. tritici.

Means in a column or group followed by the same letter(s) are not significantly different according to Fisher's Protected LSD

$(P=0.05)$. Arcsine transformation of percentage data was made in analysis to determine statistical significance. 
Table 2. Effect of fungicide treatment on disease severity and yield in wheat (WHTFOLFUN320, Suffolk, VA 2020).

\begin{tabular}{|c|c|c|c|c|c|}
\hline \multirow[b]{2}{*}{$\begin{array}{l}\text { Treatment, rate/A } \\
\text { and application timing }(\text { Feekes })^{\mathrm{z}}\end{array}$} & \multicolumn{2}{|c|}{$\begin{array}{c}\text { Scab } \\
15 \text { May }\end{array}$} & \multirow[b]{2}{*}{$\begin{array}{l}\text { Yield }^{\mathrm{w}} \\
(\mathrm{bu} / \mathrm{A})\end{array}$} & \multirow[b]{2}{*}{$\begin{array}{c}\text { Test weight } \\
(\mathrm{lb} / \mathrm{bu})\end{array}$} & \multirow[b]{2}{*}{$\%$ FDK $^{\mathrm{v}}$} \\
\hline & $\begin{array}{c}\% \\
\text { severity } \\
\end{array}$ & $\begin{array}{c}\% \\
\text { incidence }^{\mathrm{x}} \\
\end{array}$ & & & \\
\hline 1. Untreated & 0.1 & 0.9 & 88.6 & 53.6 & $3.5 \mathrm{a}$ \\
\hline 2. Folicur $3.6 \mathrm{~F} 4 \mathrm{fl} \mathrm{oz}(\mathrm{F} 9)$ & 0.5 & 4.3 & 88.7 & 52.7 & $3.5 \mathrm{ab}$ \\
\hline 3. Tilt $3.6 \mathrm{EC} 4 \mathrm{fl} \mathrm{oz}(\mathrm{F9})$ & 0.8 & 2.2 & 91.7 & 54.4 & $2.8 \mathrm{a}-\mathrm{e}$ \\
\hline 4. Aproach Prima $2.34 \mathrm{SC} 6.8 \mathrm{fl} \mathrm{oz}(\mathrm{F} 9)$ & 0.8 & 2.5 & 88.0 & 54.0 & $1.5 \mathrm{c}-\mathrm{e}$ \\
\hline 5. Priaxor $4.17 \mathrm{SC} 4 \mathrm{fl} \mathrm{oz}(\mathrm{F9})$ & 0.3 & 2.9 & 106.0 & 54.2 & $3.3 \mathrm{a}-\mathrm{c}$ \\
\hline 6. Quilt Xcel 2.2 SE $10.5 \mathrm{fl} \mathrm{oz} \mathrm{(F9)}$ & 0.3 & 3.5 & 88.8 & 53.3 & $3.3 \mathrm{a}-\mathrm{c}$ \\
\hline 7. Stratego YLD $4 \mathrm{fl} \mathrm{oz} \mathrm{(F9)}$ & 0.3 & 2.6 & 89.8 & 54.2 & $2.0 \mathrm{~b}-\mathrm{e}$ \\
\hline $\begin{array}{l}\text { 8. Priaxor 4.17 SC } 4 \mathrm{fl} \mathrm{oz} \\
+ \text { Tilt 3.6 EC } 4 \mathrm{fl} \mathrm{oz} \mathrm{(F9)}\end{array}$ & 0.1 & 1.5 & 96.9 & 54.4 & $1.0 \mathrm{e}$ \\
\hline 9. Caramba 0.75 SL $13.5 \mathrm{fl} \mathrm{oz}(\mathrm{F} 10.5 .1)$ & 0.3 & 3.8 & 93.2 & 53.5 & $3.0 \mathrm{a}-\mathrm{d}$ \\
\hline 10. Folicur $3.6 \mathrm{~F} 4 \mathrm{fl} \mathrm{oz}(\mathrm{F} 10.5 .1)$ & 1.2 & 4.2 & 99.0 & 53.9 & $4.0 \mathrm{a}$ \\
\hline 11. Proline $480 \mathrm{SC} 5.7 \mathrm{fl} \mathrm{oz}(\mathrm{F} 10.5 .1)$ & 0.8 & 2.8 & 97.3 & 54.8 & $2.8 \mathrm{a}-\mathrm{e}$ \\
\hline 12. Prosaro $421 \mathrm{SC} 6.5 \mathrm{fl} \mathrm{oz}(\mathrm{F} 10.5 .1)$ & 0.1 & 1.4 & 95.0 & 53.6 & $3.3 \mathrm{a}-\mathrm{c}$ \\
\hline 13. Tilt $3.6 \mathrm{EC} 4 \mathrm{fl} \mathrm{oz}(\mathrm{F} 10.5 .1)$ & 0.5 & 5.1 & 94.3 & 53.7 & $1.3 \mathrm{de}$ \\
\hline 14. Miravis ACE SE $13.7 \mathrm{fl} \mathrm{oz} \mathrm{(F10.5.1)}$ & 1.4 & 3.4 & 101.5 & 55.2 & $2.3 \mathrm{a}-\mathrm{e}$ \\
\hline$P(\mathrm{~F})$ & 0.71 & 0.58 & 0.28 & 0.10 & $\mathbf{0 . 0 3}$ \\
\hline LSD & N.S. & N.S. & N.S. & N.S. & 1.8 \\
\hline
\end{tabular}

${ }^{\mathrm{z}}$ Fungicide sprays were applied at Feekes 9 on 11 Apr and Feekes 10.5.1 (flowering) on 28 Apr. All fungicide treatments were applied with Induce $0.25 \% \mathrm{v} / \mathrm{v}$.

y Percent of grain heads with signs and symptoms of Fusarium head blight.

$x$ Percent of spikelets with signs and symptoms of Fusarium head blight.

w Yields are weight of wheat with $13.5 \%$ moisture. One bushel equals $60 \mathrm{lbs}$. Wheat was harvested on $24 \mathrm{Jun}$.

$\checkmark$ Percent Fusarium damaged kernels. FDK rating scale: $0=$ no damage, $100=100 \%$ Fusarium damaged kernels in scabby wheat; FDK scale by Engle, De Wolf \& Lipps, Ohio State.

Means in a column followed by the same letter(s) are not significantly different according to Fisher's Protected LSD $(P=0.05)$.

Arcsine transformation of percentage data was made in analysis to determine statistical significance. 
TEST ID: BARSCAB120

PURPOSE: To evaluate the combined effects of fungicide treatment and genetic resistance on FHB in malt barley

LOCATION: Tidewater AREC, 6321 Holland Rd., Suffolk, VA

CROP INFORMATION:

\begin{tabular}{|l|l|}
\hline Field & $61 \mathrm{~B}$ \\
\hline Crop history & 2019 corn, 2018 sorghum, 2017 wheat/sorghum \\
\hline Planting date & 31 Oct 2019 \\
\hline Variety & Flavia, Violetta, VA16M-81, VA16M-84 \\
\hline Plot length/width & $9^{\prime}$ \\
\hline Number of rows & 7 \\
\hline Row spacing & $6.67^{\prime \prime}$ \\
\hline Alleys (length between blocks) & $9^{\prime}$ \\
\hline Harvest date & 4 Jun \\
\hline
\end{tabular}

EXPERIMENTAL DESIGN: Randomized complete block with four replicates

INOCULUM: Fusarium graminearum conidia $(\sim 50,000 / \mathrm{ml})$ applied 24 hours following the Feekes 10.5 fungicide treatment with Lee Spider Sprayer; $1 \mathrm{~L}$ inoculum to $11 \mathrm{~L} \mathrm{H}_{2} \mathrm{O}$

TREATMENT APPLICATION:

\begin{tabular}{|l|l|}
\hline Equipment & Backpack sprayer \\
\hline Pressure (psi) & $38 \mathrm{psi}$ \\
\hline Nozzle type & Twinjet 8002VS \\
\hline Volume (gal/A) & 19.88 \\
\hline Surfactant & NIS $0.125 \% \mathrm{v} / \mathrm{v}$ \\
\hline
\end{tabular}

TREATMENTS:

\begin{tabular}{|c|c|c|c|c|c|}
\hline Trt \# & Variety & Fungicide and formulation & Rate, fl oz/A & App. timing & App. date \\
\hline 1 & Flavia & $\begin{array}{l}\text { Untreated } \\
\text { Inoculum }\end{array}$ & -- & $\begin{array}{l}-- \\
\text { Feekes } 10.5+1 d\end{array}$ & 17 Apr \\
\hline 2 & Flavia & $\begin{array}{l}\text { Prosaro } 421 \mathrm{SC} \\
\text { Inoculum }\end{array}$ & 6.5 & $\begin{array}{l}\text { Feekes } 10.5 \\
\text { Feekes } 10.5+1 \mathrm{~d}\end{array}$ & $\begin{array}{l}16 \mathrm{Apr} \\
17 \mathrm{Apr}\end{array}$ \\
\hline 3 & Flavia & $\begin{array}{l}\text { Miravis Ace } \\
\text { Inoculum } \\
\end{array}$ & 13.7 & $\begin{array}{l}\text { Feekes } 10.5 \\
\text { Feekes } 10.5+1 \mathrm{~d}\end{array}$ & $\begin{array}{l}16 \mathrm{Apr} \\
17 \mathrm{Apr}\end{array}$ \\
\hline 4 & Flavia & $\begin{array}{l}\text { Miravis Ace } \\
\text { Inoculum }\end{array}$ & 13.7 & $\begin{array}{l}\text { Feekes } 10.3 \\
\text { Feekes } 10.5+1 \mathrm{~d}\end{array}$ & $\begin{array}{l}11 \mathrm{Apr} \\
17 \mathrm{Apr}\end{array}$ \\
\hline 5 & Flavia & $\begin{array}{l}\text { Miravis Ace } \\
\text { Inoculum } \\
\text { Tebuconazole } 3.6 \mathrm{~F}\end{array}$ & $\begin{array}{l}13.7 \\
4 \\
\end{array}$ & $\begin{array}{l}\text { Feekes } 10.5 \\
\text { Feekes } 10.5+1 \mathrm{~d} \\
\text { Feekes } 10.5+4-6 \mathrm{~d}\end{array}$ & $\begin{array}{l}16 \mathrm{Apr} \\
17 \mathrm{Apr} \\
21 \mathrm{Apr}\end{array}$ \\
\hline 6 & Flavia & $\begin{array}{l}\text { Miravis Ace } \\
\text { Inoculum }\end{array}$ & 13.7 & $\begin{array}{l}\text { Feekes } 10.5+4-6 \mathrm{~d} \\
\text { Feekes } 10.5+1 \mathrm{~d}\end{array}$ & $\begin{array}{l}21 \mathrm{Apr} \\
17 \mathrm{Apr}\end{array}$ \\
\hline 7 & Violetta & $\begin{array}{l}\text { Untreated } \\
\text { Inoculum }\end{array}$ & -- & $\begin{array}{l}- \\
\text { Feekes } 10.5+1 \mathrm{~d}\end{array}$ & $17 \mathrm{Apr}$ \\
\hline 8 & Violetta & $\begin{array}{l}\text { Prosaro } 421 \mathrm{SC} \\
\text { Inoculum }\end{array}$ & 6.5 & $\begin{array}{l}\text { Feekes } 10.5 \\
\text { Feekes } 10.5+1 \mathrm{~d}\end{array}$ & $\begin{array}{l}16 \mathrm{Apr} \\
17 \mathrm{Apr}\end{array}$ \\
\hline 9 & Violetta & $\begin{array}{l}\text { Miravis Ace } \\
\text { Inoculum }\end{array}$ & 13.7 & $\begin{array}{l}\text { Feekes } 10.5 \\
\text { Feekes } 10.5+1 \mathrm{~d}\end{array}$ & $\begin{array}{l}16 \mathrm{Apr} \\
17 \mathrm{Apr}\end{array}$ \\
\hline 10 & Violetta & $\begin{array}{l}\text { Miravis Ace } \\
\text { Inoculum }\end{array}$ & 13.7 & $\begin{array}{l}\text { Feekes } 10.3 \\
\text { Feekes } 10.5+1 \mathrm{~d}\end{array}$ & $\begin{array}{l}11 \mathrm{Apr} \\
17 \mathrm{Apr}\end{array}$ \\
\hline 11 & Violetta & $\begin{array}{l}\text { Miravis Ace } \\
\text { Inoculum } \\
\text { Tebuconazole } 3.6 \mathrm{~F}\end{array}$ & $\begin{array}{l}13.7 \\
4\end{array}$ & $\begin{array}{l}\text { Feekes } 10.5 \\
\text { Feekes } 10.5+1 \mathrm{~d} \\
\text { Feekes } 10.5+4-6 \mathrm{~d}\end{array}$ & $\begin{array}{l}16 \mathrm{Apr} \\
17 \mathrm{Apr} \\
21 \mathrm{Apr}\end{array}$ \\
\hline 12 & Violetta & $\begin{array}{l}\text { Miravis Ace } \\
\text { Inoculum }\end{array}$ & 13.7 & $\begin{array}{l}\text { Feekes } 10.5+4-6 \mathrm{~d} \\
\text { Feekes } 10.5+1 \mathrm{~d}\end{array}$ & $\begin{array}{l}21 \mathrm{Apr} \\
17 \mathrm{Apr}\end{array}$ \\
\hline
\end{tabular}




\begin{tabular}{|c|c|c|c|c|c|}
\hline 13 & VA16M-81 & $\begin{array}{l}\text { Untreated } \\
\text { Inoculum }\end{array}$ & -- & $\begin{array}{l}-- \\
\text { Feekes } 10.5+1 \mathrm{~d}\end{array}$ & $17 \mathrm{Apr}$ \\
\hline 14 & VA16M-81 & $\begin{array}{l}\text { Prosaro } 421 \mathrm{SC} \\
\text { Inoculum }\end{array}$ & 6.5 & $\begin{array}{l}\text { Feekes } 10.5 \\
\text { Feekes } 10.5+1 \mathrm{~d}\end{array}$ & $\begin{array}{l}16 \mathrm{Apr} \\
17 \mathrm{Apr}\end{array}$ \\
\hline 15 & VA16M-81 & $\begin{array}{l}\text { Miravis Ace } \\
\text { Inoculum }\end{array}$ & 13.7 & $\begin{array}{l}\text { Feekes } 10.5 \\
\text { Feekes } 10.5+1 \mathrm{~d}\end{array}$ & $\begin{array}{l}16 \mathrm{Apr} \\
17 \mathrm{Apr}\end{array}$ \\
\hline 16 & VA16M-81 & $\begin{array}{l}\text { Miravis Ace } \\
\text { Inoculum }\end{array}$ & 13.7 & $\begin{array}{l}\text { Feekes } 10.3 \\
\text { Feekes } 10.5+1 \mathrm{~d}\end{array}$ & $\begin{array}{l}11 \mathrm{Apr} \\
17 \mathrm{Apr}\end{array}$ \\
\hline 17 & VA16M-81 & $\begin{array}{l}\text { Miravis Ace } \\
\text { Inoculum } \\
\text { Tebuconazole } 3.6 \mathrm{~F}\end{array}$ & $\begin{array}{l}13.7 \\
4 \\
\end{array}$ & $\begin{array}{l}\text { Feekes } 10.5 \\
\text { Feekes } 10.5+1 \mathrm{~d} \\
\text { Feekes } 10.5+4-6 \mathrm{~d}\end{array}$ & $\begin{array}{l}16 \mathrm{Apr} \\
17 \mathrm{Apr} \\
21 \mathrm{Apr}\end{array}$ \\
\hline 18 & VA16M-81 & $\begin{array}{l}\text { Miravis Ace } \\
\text { Inoculum }\end{array}$ & 13.7 & $\begin{array}{l}\text { Feekes } 10.5+4-6 \mathrm{~d} \\
\text { Feekes } 10.5+1 \mathrm{~d} \\
\end{array}$ & $\begin{array}{l}21 \mathrm{Apr} \\
17 \mathrm{Apr}\end{array}$ \\
\hline 19 & VA16M-84 & $\begin{array}{l}\text { Untreated } \\
\text { Inoculum }\end{array}$ & -- & $\begin{array}{l}-- \\
\text { Feekes } 10.5+1 \mathrm{~d}\end{array}$ & $17 \mathrm{Apr}$ \\
\hline 20 & VA16M-84 & $\begin{array}{l}\text { Prosaro } 421 \text { SC } \\
\text { Inoculum }\end{array}$ & 6.5 & $\begin{array}{l}\text { Feekes } 10.5 \\
\text { Feekes } 10.5+1 \mathrm{~d}\end{array}$ & $\begin{array}{l}16 \mathrm{Apr} \\
17 \mathrm{Apr}\end{array}$ \\
\hline 21 & VA16M-84 & $\begin{array}{l}\text { Miravis Ace } \\
\text { Inoculum }\end{array}$ & 13.7 & $\begin{array}{l}\text { Feekes } 10.5 \\
\text { Feekes } 10.5+1 \mathrm{~d}\end{array}$ & $\begin{array}{l}16 \mathrm{Apr} \\
17 \mathrm{Apr}\end{array}$ \\
\hline 22 & VA16M-84 & $\begin{array}{l}\text { Miravis Ace } \\
\text { Inoculum }\end{array}$ & 13.7 & $\begin{array}{l}\text { Feekes } 10.3 \\
\text { Feekes } 10.5+1 \mathrm{~d}\end{array}$ & $\begin{array}{l}11 \mathrm{Apr} \\
17 \mathrm{Apr}\end{array}$ \\
\hline 23 & VA16M-84 & $\begin{array}{l}\text { Miravis Ace } \\
\text { Inoculum } \\
\text { Tebuconazole } 3.6 \mathrm{~F}\end{array}$ & $\begin{array}{l}13.7 \\
4 \\
\end{array}$ & $\begin{array}{l}\text { Feekes } 10.5 \\
\text { Feekes } 10.5+1 \mathrm{~d} \\
\text { Feekes } 10.5+4-6 \mathrm{~d}\end{array}$ & $\begin{array}{l}16 \mathrm{Apr} \\
17 \mathrm{Apr} \\
21 \mathrm{Apr}\end{array}$ \\
\hline 24 & VA16M-84 & $\begin{array}{l}\text { Miravis Ace } \\
\text { Inoculum } \\
\end{array}$ & 13.7 & $\begin{array}{l}\text { Feekes } 10.5+4-6 \mathrm{~d} \\
\text { Feekes } 10.5+1 \mathrm{~d} \\
\end{array}$ & $\begin{array}{l}21 \mathrm{Apr} \\
17 \mathrm{Apr} \\
\end{array}$ \\
\hline
\end{tabular}

SOIL TYPE: Rains fine sandy loam

SOIL FERTILITY REPORT (Oct 2019):

\begin{tabular}{|c|c|c|c|c|c|c|c|c|c|}
\hline $\mathbf{p H}$ & $\mathbf{P}(\mathbf{l b} / \mathbf{A})$ & $\mathbf{K}(\mathbf{l b} / \mathbf{A})$ & $\mathbf{C a}(\mathbf{l b} / \mathbf{A})$ & $\mathbf{M g}(\mathbf{l b} / \mathbf{A})$ & $\mathbf{Z n}(\mathbf{p p m})$ & $\mathbf{M n}(\mathbf{p p m})$ & $\mathbf{C u}(\mathbf{p p m})$ & $\mathbf{F e}(\mathbf{p p m})$ & $\mathbf{B}(\mathbf{p p m})$ \\
\hline 5.3 & 178 & 377 & 1335 & 168 & 1.4 & 7.2 & 0.2 & 29.5 & 0.2 \\
\hline
\end{tabular}

MAINTENANCE CHEMICAL PROGRAMS:

\begin{tabular}{|l|l|}
\hline Fertilizer & Standard \\
\hline Herbicides & Standard \\
\hline Insecticides & Standard \\
\hline Fungicides & None except treatments \\
\hline Nematicides & None \\
\hline
\end{tabular}

\section{MAINTENANCE CHEMICAL APPLICATIONS:}

\begin{tabular}{|l|l|l|l|}
\hline Date & Type and target & Product and formulation & Rate/A \\
\hline $1-30-20$ & Fertility & $9-18-31$ & $322 \mathrm{lbs}$ \\
\hline $2-4-20$ & Fertility & $24-0-0-3$ & 60 units \\
\hline $3-14-20$ & Fertility & $24-0-0-3$ & 60 units \\
\hline $2-4-20$ & Herbicide & Quelex & $0.75 \mathrm{fl} \mathrm{oz}$ \\
\hline
\end{tabular}


Table 1. Effect of fungicide treatment and variety on disease incidence in malt barley (BARSCAB120, Suffolk, VA 2020).

\begin{tabular}{|c|c|c|c|c|c|}
\hline \multirow[b]{2}{*}{ Variety } & \multirow[b]{2}{*}{$\begin{array}{l}\text { Treatment, rate/A } \\
\text { and application timing }\left(\text { Feekes) }{ }^{z}\right.\end{array}$} & \multicolumn{2}{|c|}{$\begin{array}{l}\text { \% leaf blotch } \\
8 \text { May }\end{array}$} & \multicolumn{2}{|c|}{$\begin{array}{l}\text { Scab } \\
11 \text { May }\end{array}$} \\
\hline & & Flag & Flag-1 & $\%$ severity $^{x}$ & $\begin{array}{c}\% \\
\text { incidence }^{\mathrm{w}}\end{array}$ \\
\hline 1. Flavia & Untreated & 0.0 & $0.5 \mathrm{~b}-\mathrm{d}$ & $0.3 \mathrm{ef}$ & $4.8 \mathrm{e}-\mathrm{i}$ \\
\hline 2. Flavia & Prosaro 421 SC $6.5 \mathrm{fl} \mathrm{oz}(\mathrm{F} 10.5)$ & 0.0 & $0.3 \mathrm{~cd}$ & $0.5 \mathrm{c}-\mathrm{f}$ & $6.8 \mathrm{~d}-\mathrm{h}$ \\
\hline 3. Flavia & Miravis Ace $13.7 \mathrm{fl} \mathrm{oz}(\mathrm{F} 10.5)$ & 0.0 & $0.0 \mathrm{~d}$ & $0.3 \mathrm{ef}$ & $4.0 \mathrm{~g}-\mathrm{i}$ \\
\hline 4. Flavia & Miravis Ace $13.7 \mathrm{fl} \mathrm{oz}(\mathrm{F} 10.3)$ & 0.0 & $0.0 \mathrm{~d}$ & $0.0 \mathrm{f}$ & $3.8 \mathrm{f}-\mathrm{i}$ \\
\hline 5. Flavia & $\begin{array}{l}\text { Miravis Ace } 13.7 \mathrm{fl} \mathrm{oz}(\mathrm{F} 10.5) \\
\text { Tebuconazole } 3.6 \mathrm{~F} 4.0 \mathrm{fl} \mathrm{oz}(\mathrm{F} 10.5+4-6 \mathrm{~d})\end{array}$ & 0.0 & $1.3 \mathrm{~b}-\mathrm{d}$ & $0.0 \mathrm{f}$ & $2.0 \mathrm{hi}$ \\
\hline 6. Flavia & Miravis Ace $13.7 \mathrm{fl} \mathrm{oz}(\mathrm{F} 10.5+4-6 \mathrm{~d})$ & 0.0 & $1.3 \mathrm{~b}-\mathrm{d}$ & $0.0 \mathrm{f}$ & $1.8 \mathrm{i}$ \\
\hline 7. Violetta & Untreated & 0.0 & $0.5 \mathrm{~b}-\mathrm{d}$ & $0.8 \mathrm{~b}-\mathrm{e}$ & $10.5 \mathrm{c}-\mathrm{f}$ \\
\hline 8. Violetta & Prosaro 421 SC $6.5 \mathrm{fl} \mathrm{oz} \mathrm{(F10.5)}$ & 0.0 & $0.5 \mathrm{~b}-\mathrm{d}$ & $3.0 \mathrm{a}$ & $27.5 \mathrm{a}$ \\
\hline 9. Violetta & Miravis Ace $13.7 \mathrm{fl} \mathrm{oz}(\mathrm{F} 10.5)$ & 0.0 & $0.0 \mathrm{~d}$ & $2.3 \mathrm{a}$ & $19.5 \mathrm{a}-\mathrm{c}$ \\
\hline 10. Violetta & Miravis Ace $13.7 \mathrm{fl} \mathrm{oz}(\mathrm{F} 10.3)$ & 0.0 & $0.3 \mathrm{~cd}$ & $2.5 \mathrm{a}$ & $24.3 \mathrm{ab}$ \\
\hline 11. Violetta & $\begin{array}{l}\text { Miravis Ace } 13.7 \mathrm{fl} \mathrm{oz}(\mathrm{F} 10.5) \\
\text { Tebuconazole } 3.6 \mathrm{~F} 4.0 \mathrm{fl} \mathrm{oz}(\mathrm{F} 10.5+4-6 \mathrm{~d})\end{array}$ & 0.0 & $0.0 \mathrm{~d}$ & $2.0 \mathrm{ab}$ & $22.0 \mathrm{a}-\mathrm{c}$ \\
\hline 12. Violetta & Miravis Ace $13.7 \mathrm{fl} \mathrm{oz}(\mathrm{F} 10.5+4-6 \mathrm{~d})$ & 0.0 & $0.0 \mathrm{~d}$ & $1.5 \mathrm{a}-\mathrm{c}$ & $13.8 \mathrm{~b}-\mathrm{f}$ \\
\hline 13. VA168M-81 & Untreated & 0.0 & $3.0 \mathrm{a}-\mathrm{c}$ & $1.3 \mathrm{a}-\mathrm{c}$ & $15.8 \mathrm{a}-\mathrm{d}$ \\
\hline 14. VA168M-81 & Prosaro $421 \mathrm{SC} 6.5 \mathrm{fl} \mathrm{oz}(\mathrm{F} 10.5)$ & 0.0 & $2.5 \mathrm{a}-\mathrm{d}$ & $0.8 \mathrm{~b}-\mathrm{e}$ & $9.8 \mathrm{c}-\mathrm{g}$ \\
\hline 15. VA168M-81 & Miravis Ace $13.7 \mathrm{fl} \mathrm{oz}(\mathrm{F} 10.5)$ & 0.0 & $1.8 \mathrm{a}-\mathrm{d}$ & $1.5 \mathrm{a}-\mathrm{c}$ & $17.0 \mathrm{a}-\mathrm{e}$ \\
\hline 16. VA168M-81 & Miravis Ace $13.7 \mathrm{fl} \mathrm{oz}(\mathrm{F} 10.3)$ & & $0.3 \mathrm{~cd}$ & $0.3 \mathrm{ef}$ & $6.5 \mathrm{~d}-\mathrm{h}$ \\
\hline 17. VA168M-81 & $\begin{array}{l}\text { Miravis Ace } 13.7 \mathrm{fl} \mathrm{oz}(\mathrm{F} 10.5) \\
\text { Tebuconazole } 3.6 \mathrm{~F} 4.0 \mathrm{fl} \mathrm{oz}(\mathrm{F} 10.5+4-6 \mathrm{~d})\end{array}$ & 0.0 & $0.5 \mathrm{~b}-\mathrm{d}$ & $1.0 \mathrm{a}-\mathrm{d}$ & $11.8 \mathrm{~b}-\mathrm{f}$ \\
\hline 18. VA168M-81 & Miravis Ace $13.7 \mathrm{fl} \mathrm{oz}(\mathrm{F} 10.5+4-6 \mathrm{~d})$ & 0.0 & $2.8 \mathrm{a}-\mathrm{c}$ & $0.8 \mathrm{c}-\mathrm{f}$ & $7.5 \mathrm{~d}-\mathrm{i}$ \\
\hline 19. VA168M-84 & Untreated & 0.3 & $6.3 \mathrm{a}$ & $0.8 \mathrm{~b}-\mathrm{e}$ & $7.0 \mathrm{~d}-\mathrm{h}$ \\
\hline 20. VA168M-84 & Prosaro 421 SC $6.5 \mathrm{fl} \mathrm{oz}(\mathrm{F} 10.5)$ & 0.3 & $1.3 \mathrm{~b}-\mathrm{d}$ & $0.8 \mathrm{~b}-\mathrm{e}$ & $11.8 \mathrm{~b}-\mathrm{f}$ \\
\hline 21. VA168M-84 & Miravis Ace $13.7 \mathrm{fl} \mathrm{oz}(\mathrm{F} 10.5)$ & 0.3 & $4.0 \mathrm{ab}$ & $0.5 \mathrm{~d}-\mathrm{f}$ & $5.5 \mathrm{f}-\mathrm{i}$ \\
\hline 22. VA168M-84 & Miravis Ace $13.7 \mathrm{fl} \mathrm{oz}(\mathrm{F} 10.3)$ & 0.3 & $1.3 \mathrm{~b}-\mathrm{d}$ & $0.8 \mathrm{~b}-\mathrm{e}$ & $7.5 \mathrm{~d}-\mathrm{i}$ \\
\hline 23. VA168M-84 & $\begin{array}{l}\text { Miravis Ace } 13.7 \mathrm{fl} \mathrm{oz}(\mathrm{F} 10.5) \\
\text { Tebuconazole } 3.6 \mathrm{~F} 4.0 \mathrm{fl} \mathrm{oz}(\mathrm{F} 10.5+4-6 \mathrm{~d})\end{array}$ & 0.3 & $3.0 \mathrm{ab}$ & $0.5 \mathrm{c}-\mathrm{f}$ & $7.0 \mathrm{~d}-\mathrm{h}$ \\
\hline 24. VA168M-84 & Miravis Ace $13.7 \mathrm{fl} \mathrm{oz}(\mathrm{F} 10.5+4-6 \mathrm{~d})$ & 0.3 & $4.0 \mathrm{a}$ & $0.3 \mathrm{ef}$ & $5.5 \mathrm{~d}-\mathrm{i}$ \\
\hline$P(\mathrm{~F})$ & & 0.75 & 0.002 & $<0.0001$ & $<0.0001$ \\
\hline LSD & & N.S. & 3.06 & 1.14 & 9.51 \\
\hline
\end{tabular}

z Fungicide sprays were applied at Feekes 10.3 on 11 Apr, Feekes 10.5 (flowering) on 16 Apr, and Feekes 10.5 + 4-6d on 21 Apr. Fusarium inoculum (trts 1-5) was applied hours after F10.5 treatment application (17 Apr). All fungicide treatments were applied with Induce $0.125 \% \mathrm{v} / \mathrm{v}$.

y Percent of leaf area with signs and symptoms of Stagnospora nodorum blotch. Plots were also evaluated for powdery mildew and leaf rust; little to none was detected.

$x$ Percent of grain heads with signs and symptoms of Fusarium head blight.

w Percent of spikelets with signs and symptoms of Fusarium head blight.

Means in a column or group followed by the same letter(s) are not significantly different according to Fisher's Protected LSD

$(P=0.05)$. Arcsine transformation of percentage data was made in analysis to determine statistical significance. 
Table 2. Effect of fungicide treatment and variety on yield and mycotoxin levels in malt barley (BARSCAB120, Suffolk, VA 2020).

\begin{tabular}{|c|c|c|c|c|}
\hline Variety & $\begin{array}{l}\text { Treatment, rate/A } \\
\text { and application timing }(\text { Feekes })^{\mathrm{z}}\end{array}$ & $\begin{array}{l}\text { Yieldy } \\
\text { (bu/A) }\end{array}$ & $\begin{array}{l}\text { Test weight } \\
(\mathrm{lb} / \mathrm{bu})\end{array}$ & $\begin{array}{l}\text { DON } \\
\text { ppm }\end{array}$ \\
\hline 1. Flavia & Untreated & 86.2 & 51.1 & $0.62 \mathrm{a}$ \\
\hline 2. Flavia & Prosaro $421 \mathrm{SC} 6.5 \mathrm{fl} \mathrm{oz}(\mathrm{F} 10.5)$ & 83.4 & 50.5 & $0.42 \mathrm{a}-\mathrm{d}$ \\
\hline 3. Flavia & Miravis Ace $13.7 \mathrm{fl} \mathrm{oz}(\mathrm{F} 10.5)$ & 59.3 & 51.7 & $0.28 \mathrm{~cd}$ \\
\hline 4. Flavia & Miravis Ace $13.7 \mathrm{fl} \mathrm{oz}(\mathrm{F} 10.3)$ & 50.3 & 50.6 & $0.25 \mathrm{~d}$ \\
\hline 5. Flavia & $\begin{array}{l}\text { Miravis Ace } 13.7 \mathrm{fl} \mathrm{oz}(\mathrm{F} 10.5) \\
\text { Tebuconazole } 3.6 \mathrm{~F} 4.0 \mathrm{fl} \mathrm{oz}(\mathrm{F} 10.5+4-6 \mathrm{~d})\end{array}$ & 76.4 & 50.9 & $0.25 \mathrm{~d}$ \\
\hline 6. Flavia & Miravis Ace $13.7 \mathrm{fl} \mathrm{oz}(\mathrm{F} 10.5+4-6 \mathrm{~d})$ & 74.3 & 51.1 & $0.44 \mathrm{a}-\mathrm{d}$ \\
\hline 7. Violetta & Untreated & 58.3 & 51.7 & $0.20 \mathrm{~d}$ \\
\hline 8. Violetta & Prosaro $421 \mathrm{SC} 6.5 \mathrm{fl} \mathrm{oz}(\mathrm{F} 10.5)$ & 81.5 & 51.5 & $0.21 \mathrm{~d}$ \\
\hline 9. Violetta & Miravis Ace $13.7 \mathrm{fl} \mathrm{oz}(\mathrm{F} 10.5)$ & 70.3 & 52.4 & $0.25 \mathrm{~d}$ \\
\hline 10. Violetta & Miravis Ace $13.7 \mathrm{fl} \mathrm{oz}(\mathrm{F} 10.3)$ & 69.2 & 52.3 & $0.29 \mathrm{~b}-\mathrm{d}$ \\
\hline 11. Violetta & $\begin{array}{l}\text { Miravis Ace } 13.7 \mathrm{fl} \mathrm{oz}(\mathrm{F} 10.5) \\
\text { Tebuconazole } 3.6 \mathrm{~F} 4.0 \mathrm{fl} \mathrm{oz}(\mathrm{F} 10.5+4-6 \mathrm{~d})\end{array}$ & 62.3 & 53.4 & $0.21 \mathrm{~d}$ \\
\hline 12. Violetta & Miravis Ace $13.7 \mathrm{fl} \mathrm{oz}(\mathrm{F} 10.5+4-6 \mathrm{~d})$ & 65.1 & 51.4 & $0.20 \mathrm{~d}$ \\
\hline 13. VA168M-81 & Untreated & 79.7 & 51.8 & $0.51 \mathrm{a}-\mathrm{c}$ \\
\hline 14. VA168M-81 & Prosaro 421 SC $6.5 \mathrm{fl} \mathrm{oz}(\mathrm{F} 10.5)$ & 78.4 & 51.7 & $0.53 \mathrm{ab}$ \\
\hline 15. VA168M-81 & Miravis Ace $13.7 \mathrm{fl} \mathrm{oz}(\mathrm{F} 10.5)$ & 67.6 & 53.1 & $0.29 \mathrm{~b}-\mathrm{d}$ \\
\hline 16. VA168M-81 & Miravis Ace $13.7 \mathrm{fl} \mathrm{oz}(\mathrm{F} 10.3)$ & 98.5 & 52.3 & $0.58 \mathrm{a}$ \\
\hline 17. VA168M-81 & $\begin{array}{l}\text { Miravis Ace } 13.7 \mathrm{fl} \mathrm{oz}(\mathrm{F} 10.5) \\
\text { Tebuconazole } 3.6 \mathrm{~F} 4.0 \mathrm{fl} \mathrm{oz}(\mathrm{F} 10.5+4-6 \mathrm{~d})\end{array}$ & 64.5 & 45.8 & $0.29 \mathrm{~b}-\mathrm{d}$ \\
\hline 18. VA168M-81 & Miravis Ace $13.7 \mathrm{fl} \mathrm{oz}(\mathrm{F} 10.5+4-6 \mathrm{~d})$ & 64.4 & 52.6 & $0.39 \mathrm{a}-\mathrm{d}$ \\
\hline 19. VA168M-84 & Untreated & 82.0 & 53.6 & $0.62 \mathrm{a}$ \\
\hline 20. VA168M-84 & Prosaro 421 SC $6.5 \mathrm{fl} \mathrm{oz}(\mathrm{F} 10.5)$ & 57.3 & 53.9 & $0.27 \mathrm{~cd}$ \\
\hline 21. VA168M-84 & Miravis Ace $13.7 \mathrm{fl} \mathrm{oz}(\mathrm{F} 10.5)$ & 71.9 & 54.1 & $0.33 \mathrm{~b}-\mathrm{d}$ \\
\hline 22. VA168M-84 & Miravis Ace $13.7 \mathrm{fl} \mathrm{oz}(\mathrm{F} 10.3)$ & 52.7 & 54.0 & $0.29 \mathrm{~b}-\mathrm{d}$ \\
\hline 23. VA168M-84 & $\begin{array}{l}\text { Miravis Ace } 13.7 \mathrm{fl} \mathrm{oz}(\mathrm{F} 10.5) \\
\text { Tebuconazole } 3.6 \mathrm{~F} 4.0 \mathrm{fl} \mathrm{oz}(\mathrm{F} 10.5+4-6 \mathrm{~d})\end{array}$ & 58.1 & 53.2 & $0.22 \mathrm{~d}$ \\
\hline 24. VA168M-84 & Miravis Ace $13.7 \mathrm{fl} \mathrm{oz}(\mathrm{F} 10.5+4-6 \mathrm{~d})$ & 60.5 & 53.0 & $0.22 \mathrm{~d}$ \\
\hline$P(\mathrm{~F})$ & & 0.35 & 0.11 & 0.001 \\
\hline LSD & & N.S. & N.S. & 3.89 \\
\hline
\end{tabular}

z Fungicide sprays were applied at Feekes 10.3 on 11 Apr, Feekes 10.5 (flowering) on 16 Apr, and Feekes 10.5 + 4-6d on 21 Apr. Fusarium inoculum (trts 1-5) was applied hours after F10.5 treatment application (17 Apr). All fungicide treatments were applied with Induce $0.125 \% \mathrm{v} / \mathrm{v}$.

y Yields are weight of barley with $13.5 \%$ moisture. One bushel equals $48 \mathrm{lbs}$. Barley was harvested on 4 Jun.

Means in a column or group followed by the same letter(s) are not significantly different according to Fisher's Protected LSD $(P=0.05)$. 
TEST ID: BARSCAB220

PURPOSE: To evaluate foliar treatment and application timing for FHB control in malt barley and impact on yield

LOCATION: Tidewater Research Farm, Hare Rd., Suffolk, VA

CROP INFORMATION:

\begin{tabular}{|l|l|}
\hline Field & 9B \\
\hline Crop history & 2019 wheat/soy, 2018 corn, 2017 wheat/soy \\
\hline Planting date & 22 Nov 2019 \\
\hline Variety & Thoroughbred \\
\hline Seeding rate & 30 seed/ft \\
\hline Plot length/width & $30^{\prime} \times 5^{\prime}$ '(treat \& harvest), 30' x 12' total \\
\hline Number of rows & 8 \\
\hline Row spacing & $7.5^{\prime}$ \\
\hline Alleys (length between blocks) & $9^{\prime}$ \\
\hline Harvest date & 9 Jun \\
\hline
\end{tabular}

EXPERIMENTAL DESIGN: Randomized complete block with four replicates

INOCULUM: Fusarium graminearum conidia ( 50,000/ml) applied 24 hours following the Feekes 10.5 fungicide treatment with Lee Spider Sprayer; $1 \mathrm{~L}$ inoculum to $11 \mathrm{~L} \mathrm{H}_{2} \mathrm{O}$

TREATMENT APPLICATION:

\begin{tabular}{|l|l|}
\hline Equipment & Lee Spider sprayer \\
\hline Pressure (psi) & $38 \mathrm{psi}$ \\
\hline Nozzle type & Twinjet $8002 \mathrm{VS}$ \\
\hline Volume (gal/A) & 19.88 \\
\hline Surfactant & NIS $0.125 \% \mathrm{v} / \mathrm{v}$ \\
\hline
\end{tabular}

TREATMENTS:

\begin{tabular}{|c|c|c|c|c|}
\hline Trt \# & Fungicide and formulation & Rate, fl oz/A & App. timing & App. date \\
\hline 1 & $\begin{array}{l}\text { Untreated } \\
\text { Inoculum }\end{array}$ & -- & $\begin{array}{l}-- \\
\text { Feekes } 10.5+1 d\end{array}$ & $22 \mathrm{Apr}$ \\
\hline 2 & $\begin{array}{l}\text { Prosaro } 421 \mathrm{SC} \\
\text { Inoculum }\end{array}$ & 6.5 & $\begin{array}{l}\text { Feekes } 10.5 \\
\text { Feekes } 10.5+1 \mathrm{~d} \\
\end{array}$ & $\begin{array}{l}21 \mathrm{Apr} \\
22 \mathrm{Apr}\end{array}$ \\
\hline 3 & $\begin{array}{l}\text { Caramba } 0.75 \mathrm{SL} \\
\text { Inoculum }\end{array}$ & 13.5 & $\begin{array}{l}\text { Feekes } 10.5 \\
\text { Feekes } 10.5+1 \mathrm{~d}\end{array}$ & $\begin{array}{l}21 \mathrm{Apr} \\
22 \mathrm{Apr}\end{array}$ \\
\hline 4 & $\begin{array}{l}\text { Miravis Ace SE } \\
\text { Inoculum }\end{array}$ & 13.7 & $\begin{array}{l}\text { Feekes } 10.3 \\
\text { Feekes } 10.5+1 \mathrm{~d}\end{array}$ & $\begin{array}{l}16 \mathrm{Apr} \\
22 \mathrm{Apr}\end{array}$ \\
\hline 5 & $\begin{array}{l}\text { Miravis Ace SE } \\
\text { Inoculum }\end{array}$ & 13.7 & $\begin{array}{l}\text { Feekes } 10.5 \\
\text { Feekes } 10.5+1 \mathrm{~d}\end{array}$ & $\begin{array}{l}21 \mathrm{Apr} \\
22 \mathrm{Apr}\end{array}$ \\
\hline 6 & $\begin{array}{l}\text { Miravis Ace SE } \\
\text { Inoculum }\end{array}$ & 13.7 & $\begin{array}{l}\text { Feekes } 10.5+4-6 \mathrm{~d} \\
\text { Feekes } 10.5+1 \mathrm{~d} \\
\end{array}$ & $\begin{array}{l}28 \mathrm{Apr} \\
22 \mathrm{Apr}\end{array}$ \\
\hline 7 & $\begin{array}{l}\text { Miravis Ace SE } \\
\text { Inoculum } \\
\text { Prosaro } 421 \mathrm{SC}\end{array}$ & $\begin{array}{l}13.7 \\
6.5 \\
\end{array}$ & $\begin{array}{l}\text { Feekes } 10.5 \\
\text { Feekes } 10.5+1 \mathrm{~d} \\
\text { Feekes } 10.5+4-6 \mathrm{~d}\end{array}$ & $\begin{array}{l}21 \mathrm{Ap} \\
22 \mathrm{Apr} \\
28 \mathrm{Apr}\end{array}$ \\
\hline 8 & $\begin{array}{l}\text { Miravis Ace SE } \\
\text { Inoculum } \\
\text { Caramba } 0.75 \mathrm{SL} \\
\end{array}$ & $\begin{array}{l}13.7 \\
13.5 \\
\end{array}$ & $\begin{array}{l}\text { Feekes } 10.5 \\
\text { Feekes } 10.5+1 \mathrm{~d} \\
\text { Feekes } 10.5+4-6 \mathrm{~d}\end{array}$ & $\begin{array}{l}21 \mathrm{Apr} \\
22 \mathrm{Apr} \\
28 \mathrm{Apr}\end{array}$ \\
\hline 9 & $\begin{array}{l}\text { Miravis Ace SE } \\
\text { Inoculum } \\
\text { Tebuconazole } 3.6 \mathrm{~F}\end{array}$ & $\begin{array}{l}13.7 \\
4 \\
\end{array}$ & $\begin{array}{l}\text { Feekes } 10.5 \\
\text { Feekes } 10.5+1 \mathrm{~d} \\
\text { Feekes } 10.5+4-6 \mathrm{~d}\end{array}$ & $\begin{array}{l}21 \mathrm{Apr} \\
22 \mathrm{Apr} \\
28 \mathrm{Apr}\end{array}$ \\
\hline 10 & $\begin{array}{l}\text { Prosaro } 421 \mathrm{SC} \\
\text { Inoculum }\end{array}$ & 6.5 & $\begin{array}{l}\text { Feekes } 10.3 \\
\text { Feekes } 10.5+1 \mathrm{~d}\end{array}$ & $\begin{array}{l}16 \mathrm{Apr} \\
22 \mathrm{Apr}\end{array}$ \\
\hline 11 & $\begin{array}{l}\text { Prosaro 421 SC } \\
\text { Inoculum }\end{array}$ & 6.5 & $\begin{array}{l}\text { Feekes } 10.5+4-6 \mathrm{~d} \\
\text { Feekes } 10.5+1 \mathrm{~d} \\
\end{array}$ & $\begin{array}{l}28 \mathrm{Apr} \\
22 \mathrm{Apr} \\
\end{array}$ \\
\hline
\end{tabular}




\begin{tabular}{|l|l|l|l|l|}
\hline 12 & $\begin{array}{l}\text { Caramba 0.75 SL } \\
\text { Inoculum }\end{array}$ & 13.5 & $\begin{array}{l}\text { Feekes 10.3 } \\
\text { Feekes 10.5 +1d }\end{array}$ & $\begin{array}{l}16 \mathrm{Apr} \\
22 \mathrm{Apr}\end{array}$ \\
\hline 13 & $\begin{array}{l}\text { Caramba 0.75 SL } \\
\text { Inoculum }\end{array}$ & 13.5 & $\begin{array}{l}\text { Feekes 10.5 +4-6d } \\
\text { Feekes 10.5 +1d }\end{array}$ & $\begin{array}{l}28 \mathrm{Apr} \\
22 \mathrm{Apr}\end{array}$ \\
\hline
\end{tabular}

SOIL TYPE: Suffolk loamy sand

SOIL FERTILITY REPORT (Oct 2019):

\begin{tabular}{|l|c|c|c|c|c|c|c|c|c|}
\hline $\mathbf{p H}$ & $\mathbf{P}(\mathbf{l b} / \mathbf{A})$ & $\mathbf{K}(\mathbf{l b} / \mathbf{A})$ & $\mathbf{C a}(\mathbf{l b} / \mathbf{A})$ & $\mathbf{M g}(\mathbf{l b} / \mathbf{A})$ & $\mathbf{Z n}(\mathbf{p p m})$ & $\mathbf{M n}(\mathbf{p p m})$ & $\mathbf{C u}(\mathbf{p p m})$ & $\mathbf{F e}(\mathbf{p p m})$ & $\mathbf{B}(\mathbf{p p m})$ \\
\hline 5.7 & 102 & 50 & 642 & 92 & 0.6 & 4.6 & 0.4 & 19.3 & 0.1 \\
\hline
\end{tabular}

MAINTENANCE CHEMICAL PROGRAMS:

\begin{tabular}{|l|l|}
\hline Fertilizer & Standard \\
\hline Herbicides & Standard \\
\hline Insecticides & Standard \\
\hline Fungicides & None except treatments \\
\hline Nematicides & None \\
\hline
\end{tabular}

MAINTENANCE CHEMICAL APPLICATIONS:

\begin{tabular}{|l|l|l|l|}
\hline Date & Type and target & Product and formulation & Rate/A \\
\hline $1-30-20$ & Fertility & $9-18-31$ & $322 \mathrm{lbs}$ \\
\hline $2-4-20$ & Fertility & $24-0-0-3$ & 60 units \\
\hline $3-14-20$ & Fertility & $24-0-0-3$ & 60 units \\
\hline $2-4-20$ & Herbicide & Quelex & $0.75 \mathrm{fl} \mathrm{oz}$ \\
\hline
\end{tabular}


Table 1. Effect of fungicide treatment on disease incidence and yield in malt barley (BARSCAB220, Suffolk, VA 2020).

\begin{tabular}{|c|c|c|c|c|c|c|}
\hline \multirow[b]{2}{*}{$\begin{array}{l}\text { Treatment, rate/A } \\
\text { and application timing (Feekes) }{ }^{\mathrm{z}}\end{array}$} & \multicolumn{2}{|c|}{$\begin{array}{c}\text { Scab } \\
13 \text { May } \\
\end{array}$} & \multicolumn{2}{|c|}{$\begin{array}{l}\text { \% leaf blotch } \\
14 \text { May }\end{array}$} & \multirow[b]{2}{*}{$\begin{array}{c}\text { Yield }^{\mathbf{v}} \\
(\text { bu/A) }\end{array}$} & \multirow{2}{*}{$\begin{array}{c}\text { Test } \\
\text { weight } \\
\text { (lb/bu) }\end{array}$} \\
\hline & $\begin{array}{c}\% \\
\text { severity }^{\mathrm{y}}\end{array}$ & $\begin{array}{c}\% \\
\text { incidence }^{\mathrm{x}} \\
\end{array}$ & Flag & Flag-1 & & \\
\hline 1. Untreated & 0.5 & 6.5 & $5.0 \mathrm{ab}$ & 18.8 & 65.8 & 48.6 \\
\hline 2. Prosaro 421 SC $6.5 \mathrm{fl} \mathrm{oz} \mathrm{(F10.5)}$ & 0.6 & 8.1 & $5.3 \mathrm{a}-\mathrm{c}$ & 16.3 & 72.4 & 49.5 \\
\hline 3. Caramba 0.75 SL $13.5 \mathrm{fl} \mathrm{oz}(\mathrm{F} 10.5)$ & 0.4 & 6.0 & $7.8 \mathrm{a}$ & 20.0 & 82.8 & 49.7 \\
\hline 4. Miravis ACE SE $13.7 \mathrm{fl} \mathrm{oz} \mathrm{(F10.3)}$ & 0.6 & 8.4 & $0.5 \mathrm{~d}$ & 6.5 & 79.7 & 49.8 \\
\hline 5. Miravis ACE SE $13.7 \mathrm{fl} \mathrm{oz} \mathrm{(F10.5)}$ & 0.3 & 4.5 & $2.0 \mathrm{~b}-\mathrm{d}$ & 10.0 & 84.4 & 50.1 \\
\hline 6. Miravis ACE SE $13.7 \mathrm{fl} \mathrm{oz}(\mathrm{F} 10.5+4-6 \mathrm{~d})$ & 0.5 & 6.7 & $1.8 \mathrm{~cd}$ & 7.5 & 88.4 & 50.5 \\
\hline $\begin{array}{l}\text { 7. Miravis ACE SE } 13.7 \mathrm{fl} \mathrm{oz} \mathrm{(F10.5)} \\
\text { Prosaro 421 SC } 6.5 \mathrm{fl} \mathrm{oz}(\text { F10.5 + 4-6d) }\end{array}$ & 0.6 & 7.8 & $3.0 \mathrm{a}-\mathrm{d}$ & 13.8 & 87.6 & 50.3 \\
\hline $\begin{array}{l}\text { 8. Miravis ACE SE } 13.7 \mathrm{fl} \mathrm{oz}(\mathrm{F} 10.5) \\
\text { Caramba 0.75 SL } 13.5 \mathrm{fl} \mathrm{oz}(\mathrm{F} 10.5+4-6 \mathrm{~d})\end{array}$ & 0.7 & 7.6 & $3.0 \mathrm{a}-\mathrm{d}$ & 10.0 & 83.1 & 51.2 \\
\hline $\begin{array}{l}\text { 9. Miravis ACE SE } 13.7 \mathrm{fl} \mathrm{oz}(\mathrm{F} 10.5) \\
\text { Tebuconazole } 3.6 \mathrm{~F} 4 \mathrm{fl} \mathrm{oz}(\mathrm{F} 10.5+4-6 \mathrm{~d})\end{array}$ & 0.5 & 4.7 & $2.0 \mathrm{~b}-\mathrm{d}$ & 11.3 & 95.3 & 50.7 \\
\hline 10. Prosaro 421 SC $6.5 \mathrm{fl} \mathrm{oz}(\mathrm{F} 10.3)$ & 0.3 & 4.3 & $4.0 \mathrm{a}-\mathrm{c}$ & 13.8 & 82.5 & 49.6 \\
\hline 11. Prosaro 421 SC $6.5 \mathrm{fl} \mathrm{oz}(\mathrm{F} 10.5+4-6 \mathrm{~d})$ & 0.4 & 4.4 & $4.0 \mathrm{a}-\mathrm{c}$ & 15.0 & 70. & 49.5 \\
\hline 12. Caramba $0.75 \mathrm{SL} 13.5 \mathrm{fl} \mathrm{oz}$ (F10.3) & 0.3 & 4.8 & $6.5 \mathrm{ab}$ & 16.3 & 70.5 & 48.4 \\
\hline 13. Caramba 0.75 SL $13.5 \mathrm{fl} \mathrm{oz}(\mathrm{F} 10.5+4-6 \mathrm{~d})$ & 0.6 & 7.3 & $6.3 \mathrm{a}$ & 15.0 & 57.8 & 49.2 \\
\hline$P(\mathrm{~F})$ & 0.93 & 0.92 & $\mathbf{0 . 0 3}$ & 0.12 & 0.10 & 0.26 \\
\hline LSD & N.S. & N.S. & 5.06 & N.S. & N.S. & N.S. \\
\hline
\end{tabular}

${ }^{\mathrm{z}}$ Fungicide sprays were applied at Feekes 3 on 16 Apr, Feekes 10.5 (flowering) on $21 \mathrm{Apr}$, and Feekes 10.5 + 4-6d on 28 Apr. Fusarium graminearum conidia $(\sim 50,000 / \mathrm{ml})$ applied on $22 \mathrm{Apr}$ to all plots 24 hours following the Feekes 10.5 fungicide treatment. All fungicide treatments applied with Induce $0.125 \% \mathrm{v} / \mathrm{v}$.

y Percent of grain heads with signs and symptoms of Fusarium head blight.

$x$ Percent of spikelets with signs and symptoms of Fusarium head blight.

${ }^{w}$ Percent of leaf area with signs and symptoms of Stagnospora nodorum blotch. Plots were also evaluated for symptoms of powdery mildew and rust; none were observed.

$\checkmark$ Yields are weight of barley with $13.5 \%$ moisture. One bushel equals $48 \mathrm{lbs}$. Barley was harvested on $9 \mathrm{Jun}$.

Means in a column or group followed by the same letter(s) are not significantly different according to Fisher's Protected LSD $(P=0.05)$. Arcsine transformation of percentage data was made in analysis to determine statistical significance. 
TEST ID: CORNNEMA220

PURPOSE: To compare nematicide seed treatments and in-furrow nematicides for impact on plant health and yield in corn

LOCATION: Tidewater AREC, 6321 Holland Rd., Suffolk, VA

CROP INFORMATION:

\begin{tabular}{|l|l|}
\hline Field & 55 \\
\hline Crop history & 2019 soybean, 2018 corn, 2017 soybean \\
\hline Planting date & 12 May \\
\hline Seeding rate & 2 seed/row ft \\
\hline Plot length/width & $30^{\prime} \times 6^{\prime}$ \\
\hline Number of rows & 2 rows \\
\hline Row spacing & $366^{\prime}$ \\
\hline Alleys (length between blocks) & $8^{\prime}$ \\
\hline Harvest date & $23 \mathrm{Sep}$ \\
\hline
\end{tabular}

EXPERIMENTAL DESIGN: Randomized complete block with four replicates

TREATMENT APPLICATION:

\begin{tabular}{|l|l|l|}
\hline & IF liquid & IF granular \\
\hline Equipment & $\mathrm{CO}_{2}$ sprayer & Noble Box \\
\hline Pressure (psi) & -- & -- \\
\hline Nozzle type & .075 microtube & -- \\
\hline Volume (gal/A) & $5 \mathrm{gal} / \mathrm{A}$ & $\mathrm{lb} / \mathrm{A}$ (trt rate) \\
\hline
\end{tabular}

TREATMENTS:

\begin{tabular}{|l|l|l|l|l|}
\hline Trt \# & Seed treatment & Seed treatment rate a.i.* & In furrow treatment & In furrow rate/A \\
\hline 1 & Base seed treatment & & -- & -- \\
\hline 2 & BioST Nematicide & $7 \mathrm{fl} \mathrm{oz} / \mathrm{cwt}$ & -- & -- \\
\hline 3 & Trunemco & $0.31 \mathrm{fl} \mathrm{oz} / \mathrm{cwt}$ & -- & -- \\
\hline 4 & Aveo & $0.1 \mathrm{fl} \mathrm{oz} / \mathrm{cwt}$ & -- & -- \\
\hline 5 & VoTIVO & $0.8 \mathrm{fl} \mathrm{oz} / \mathrm{cwt}$ & -- & -- \\
\hline 6 & Base seed treatment & & Counter WG & $5 \mathrm{lb}$ \\
\hline 7 & Base seed treatment & Luna Privilege & $6.5 \mathrm{fl} \mathrm{oz}$ \\
\hline $\begin{array}{l}\text { *Base seed treatment = Maxium Quatro } 1 \mathrm{fl} \mathrm{oz}+\text { BioST VPH } 1 \mathrm{fl} \mathrm{oz} \mathrm{+} \mathrm{Nipsit} 2.5 \mathrm{fl} \mathrm{oz} / \mathrm{cwt} \text {; Seed treated by } \\
\text { personnel at Albaugh LLC. }\end{array}$
\end{tabular}

SOIL TYPE: Nansemond fine sandy loam

SOIL FERTILITY REPORT (Jan 2020):

\begin{tabular}{|l|l|l|l|l|l|l|l|l|l|}
\hline $\mathbf{p H}$ & $\mathbf{P}(\mathbf{l b} / \mathbf{A})$ & $\mathbf{K}(\mathbf{l b} / \mathbf{A})$ & $\mathbf{C a}(\mathbf{l b} / \mathbf{A})$ & $\mathbf{M g}(\mathbf{l b} / \mathbf{A})$ & $\mathbf{Z n}(\mathbf{p p m})$ & $\mathbf{M n}(\mathbf{p p m})$ & $\mathbf{C u}(\mathbf{p p m})$ & $\mathbf{F e}(\mathbf{p p m})$ & $\mathbf{B}(\mathbf{p p m})$ \\
\hline 6.5 & 108 & 170 & 1025 & 153 & 0.9 & 3.8 & 0.6 & 45.7 & 0.2 \\
\hline
\end{tabular}


MAINTENANCE CHEMICAL PROGRAMS:

\begin{tabular}{|l|l|}
\hline Fertilizer & Standard \\
\hline Herbicides & Standard \\
\hline Insecticides & Standard \\
\hline Fungicides & None \\
\hline Nematicides & None except treatments \\
\hline
\end{tabular}

MAINTENANCE CHEMICAL APPLICATIONS:

\begin{tabular}{|l|l|l|l|}
\hline Date & Type and target & Product and formulation & Rate/A \\
\hline $5 / 13 / 20$ & Herbicide & Bicep II MAGNUM & $2 \mathrm{pt}$ \\
\hline $5 / 13 / 20$ & Herbicide & Simazine 4L & $1 \mathrm{pt}$ \\
\hline $5 / 13 / 20$ & Herbicide & Roundup WeatherMAX & $1 \mathrm{qt}$ \\
\hline
\end{tabular}

Table 1. Effect of nematicide seed treatment and in-furrow nematicides on plant emergence and plant health in corn (CORNNEMA220, Suffolk, VA 2020).

\begin{tabular}{|c|c|c|c|c|}
\hline \multirow[b]{2}{*}{ Seed treatment and rate ${ }^{z}$} & \multirow{2}{*}{$\begin{array}{l}\text { In-furrow treatment } \\
\text { and rate/Az }\end{array}$} & \multicolumn{2}{|c|}{ Plants/ft. ${ }^{\mathbf{y}}$} & \multirow{2}{*}{$\begin{array}{l}\text { Vigor, 1-10 } \\
18 \text { Jun } \\
\end{array}$} \\
\hline & & 21 May & 9 Jun & \\
\hline 1. Base seed treatment & -- & 1.3 & 1.3 & $9.6 \mathrm{a}$ \\
\hline 2. BioST Nematicide $6 \mathrm{fl} \mathrm{oz} / \mathrm{cwt}$ & -- & 1.3 & 1.3 & $9.8 \mathrm{a}$ \\
\hline 3. Trunemco $0.31 \mathrm{fl} \mathrm{oz} / \mathrm{cwt}$ & -- & 1.2 & 1.3 & $9.5 \mathrm{a}$ \\
\hline 4. Aveo $0.1 \mathrm{fl} \mathrm{oz} / \mathrm{cwt}$ & -- & 1.3 & 1.3 & $9.4 \mathrm{a}$ \\
\hline 5. VoTIVO $0.8 \mathrm{fl} \mathrm{oz} / \mathrm{cwt}$ & -- & 1.3 & 1.3 & $9.5 \mathrm{a}$ \\
\hline 6. Base seed treatment & Counter WG $5 \mathrm{lb}$ & 1.2 & 1.2 & $8.0 \mathrm{~b}$ \\
\hline 7. Base seed treatment & Luna Privilege $6.5 \mathrm{fl} \mathrm{oz}$ & 1.2 & 1.3 & $9.4 \mathrm{a}$ \\
\hline$P(\mathrm{~F})$ & & 0.16 & 0.53 & 0.002 \\
\hline LSD & & N.S. & N.S. & 0.77 \\
\hline
\end{tabular}

z Base seed treatment = Maxium Quatro $1 \mathrm{fl} \mathrm{oz}+$ BioST VPH $1 \mathrm{fl} \mathrm{oz}+$ Nipsit $2.5 \mathrm{fl} \mathrm{oz} / \mathrm{cwt}$. Seed treated by personnel at Albaugh LLC. In-furrow treatments applied at planting (12 May).

y Determined from counts in two, 30 -ft rows per plot.

$x$ Vigor index rating scale: $10=100 \%$ vigor, $1=$ no vigor.

Means in a column followed by the same letter(s) are not significantly different according to Fisher's Protected LSD ( $\mathrm{P}=0.05)$. 
Table 2. Effect of nematicide seed treatment and in-furrow nematicides on yield in corn (CORNNEMA220, Suffolk, VA 2020).

\begin{tabular}{|l|l|c|c|}
\hline Seed treatment and rate & \multicolumn{1}{|c|}{$\begin{array}{l}\text { In-furrow treatment } \\
\text { and rate/A }\end{array}$} & $\begin{array}{c}\text { Yield } \\
(\mathbf{b u} / \mathbf{A})\end{array}$ & $\begin{array}{c}\text { Test weight } \\
(\mathbf{l b} / \mathbf{b u})\end{array}$ \\
\hline 1. Base seed treatment & -- & 189.4 & 52.7 \\
\hline 2. BioST Nematicide 6 fl oz/cwt & -- & 191.3 & 52.6 \\
\hline 3. Trunemco 0.31 fl oz/cwt & -- & 180.1 & 52.6 \\
\hline 4. Aveo 0.1 fl oz/cwt & -- & 187.0 & 51.8 \\
\hline 5. VoTIVO 0.8 fl oz/cwt & -- & 189.0 & 52.4 \\
\hline 6. Base seed treatment & Counter WG 5 lb & 172.7 & 51.6 \\
\hline 7. Base seed treatment & Luna Privilege 6.5 fl oz & 189.6 & 51.8 \\
\hline$P($ F) & & 0.76 & 0.42 \\
\hline LSD & & N.S. & N.S. \\
\hline
\end{tabular}

${ }_{\mathrm{z}}$ Base seed treatment $=$ Maxium Quatro $1 \mathrm{fl} \mathrm{oz}+$ BioST VPH $1 \mathrm{fl} \mathrm{oz}+$ Nipsit $2.5 \mathrm{fl}$ oz/cwt. Seed treated by personnel at Albaugh LLC. In-furrow treatments applied at planting (12 May).

y Yields are weight of corn with moisture content of $15.5 \%$. Corn was harvested on $23 \mathrm{Sep}$. One bushel $=56 \mathrm{lbs}$ of grain. 
TEST ID: CORNFOLFUN120

PURPOSE: To evaluate fungicides and spray timing for control of foliar disease in corn and impact on yield at two locations, site 1

LOCATION: Tidewater AREC Duke Farm, Holland Rd., Suffolk, VA

CROP INFORMATION:

\begin{tabular}{|l|l|}
\hline Field & 45 \\
\hline Crop history & 2019 soybean/wheat, 2018 corn, 2017 soybean/wheat \\
\hline Planting date & 6 May \\
\hline Variety & DKC62-05 \\
\hline Seeding rate & 2 seed/row ft \\
\hline Plot length/width & $30^{\prime} \times 12^{\prime}$ \\
\hline Number of rows & 4 \\
\hline Row spacing & $36^{\prime \prime}$ \\
\hline Alleys (length between blocks) & $8^{\prime}$ \\
\hline Harvest date & 5 Oct \\
\hline
\end{tabular}

EXPERIMENTAL DESIGN: Randomized complete block with four replicates

TREATMENT APPLICATION:

\begin{tabular}{|l|l|}
\hline Equipment & Lee Spider Sprayer \\
\hline Pressure (psi) & 38 psi \\
\hline Nozzle type & Twinjet 8002 VS \\
\hline Volume (gal/A) & 19.88 \\
\hline Surfactant & NIS $0.25 \%$ v/v \\
\hline
\end{tabular}

\section{TREATMENTS:}

\begin{tabular}{|c|l|c|c|l|}
\hline Trt \# & Fungicide and formulation & Rate, fl oz/A & App. timing & App. date \\
\hline 1 & Untreated control & & & \\
\hline 2 & Tilt 3.6 EC & 4 & V5 & $23 \mathrm{Jun}$ \\
\hline 3 & Tilt 3.6 EC & 4 & VT/R1 & $20 \mathrm{Jul}$ \\
\hline 4 & Affiance & 10 & V5 & $23 \mathrm{Jun}$ \\
\hline 5 & Affiance & 10 & VT/R1 & $20 \mathrm{Jul}$ \\
\hline 6 & Headline AMP & 10 & V5 & $23 \mathrm{Jun}$ \\
\hline 7 & Headline AMP & 10 & VT/R1 & $20 \mathrm{Jul}$ \\
\hline 8 & Miravis Neo 2.5 SE & 13.7 & V5 & $23 \mathrm{Jun}$ \\
\hline 9 & Miravis Neo 2.5 SE & 13.7 & VT/R1 & $20 \mathrm{Jul}$ \\
\hline 10 & Veltyma & 7 & V5 & $23 \mathrm{Jun}$ \\
\hline 11 & Veltyma & 7 & VT/R1 & $20 \mathrm{Jul}$ \\
\hline 12 & Lucento 4.17 SC & 5 & V5 & $23 \mathrm{Jun}$ \\
\hline 13 & Lucento 4.17 SC & 5 & VT/R1 & $20 \mathrm{Jul}$ \\
\hline 14 & Topguard EQ 4.29 SC & 7 & V5 & $23 \mathrm{Jun}$ \\
\hline 15 & Topguard EQ 4.29 SC & 6 & V7 & $8 \mathrm{Jul}$ \\
\hline 16 & Topguard EQ 4.29 SC & 5 & VT/R1 & $20 \mathrm{Jul}$ \\
\hline 17 & USF0411 SC & 8 & V5 & $23 \mathrm{Jun}$ \\
\hline 18 & USF0411 SC & 8 & VT/R1 & $20 \mathrm{Jul}$ \\
\hline 19 & Stratego YLD & 4 & V5 & $23 \mathrm{Jun}$ \\
\hline 20 & Stratego YLD & 4 & VT/R1 & $20 \mathrm{Jul}$ \\
\hline & & & & \\
\hline
\end{tabular}


SOIL TYPE: Dragston fine sandy loam

SOIL FERTILITY REPORT (Jan 2020):

\begin{tabular}{|l|l|l|l|l|l|l|l|l|l|}
\hline $\mathbf{p H}$ & $\mathbf{P}(\mathbf{l b} / \mathbf{A})$ & $\mathbf{K}(\mathbf{l b} / \mathbf{A})$ & $\mathbf{C a}(\mathbf{l b} / \mathbf{A})$ & $\mathbf{M g}(\mathbf{l b} / \mathbf{A})$ & $\mathbf{Z n}(\mathbf{p p m})$ & $\mathbf{M n}(\mathbf{p p m})$ & $\mathbf{C u}(\mathbf{p p m})$ & $\mathbf{F e}(\mathbf{p p m})$ & $\mathbf{B}(\mathbf{p p m})$ \\
\hline 6.0 & 50 & 165 & 621 & 96 & 0.4 & 28 & 0.3 & 32.4 & 0.1 \\
\hline
\end{tabular}

MAINTENANCE CHEMICAL PROGRAMS:

\begin{tabular}{|l|l|}
\hline Fertilizer & Standard \\
\hline Herbicides & Standard \\
\hline Insecticides & Standard \\
\hline Fungicides & None except treatments \\
\hline Nematicides & Standard \\
\hline
\end{tabular}

MAINTENANCE CHEMICAL APPLICATIONS:

\begin{tabular}{|l|l|l|l|}
\hline Date & Type and target & Product and formulation & Rate/A \\
\hline $3 / 26 / 20$ & Herbicide & Roundup WeatherMAX & $1 \mathrm{qt}$ \\
\hline $3 / 26 / 20$ & Herbicide & Liberty 280SL & $1 \mathrm{qt}$ \\
\hline $3 / 26 / 20$ & Herbicide & $2,4-D$ & $1 \mathrm{pt}$ \\
\hline $5 / 13 / 20$ & Herbicide & Bicep II MAGNUM & $2 \mathrm{pt}$ \\
\hline $5 / 13 / 20$ & Herbicide & Simazine 4L & $1 \mathrm{pt}$ \\
\hline $5 / 13 / 20$ & Herbicide & Roundup WeatherMAX & $1 \mathrm{qt}$ \\
\hline $6 / 4 / 20$ & Herbicide & Halex GT & $4 \mathrm{pt}$ \\
\hline $6 / 4 / 20$ & Herbicide & Atrazine & $1.5 \mathrm{pt}$ \\
\hline
\end{tabular}


Table 1. Effect of fungicide treatment and application timing on foliar disease in corn (CORNFOLFUN120, Suffolk, VA 2020).

\begin{tabular}{|c|c|c|c|c|}
\hline \multirow[b]{2}{*}{ Treatment, rate/A and timing ${ }^{\mathrm{z}}$} & \multicolumn{3}{|c|}{$\%$ foliar disease ${ }^{y}$} & \multirow[b]{2}{*}{$\begin{array}{c}\text { Plant health } \\
(1-10)^{x} \\
14 \text { Aug }\end{array}$} \\
\hline & 14 Aug & $\begin{array}{c}\text { Gray leaf spot } \\
4 \text { Sep }\end{array}$ & $\begin{array}{c}\text { Curvularia } \\
\text { leaf spot } \\
4 \text { Sep }\end{array}$ & \\
\hline 1. Untreated & 1.0 & 7.5 & 18.8 & 9.3 \\
\hline 2. Tilt $3.6 \mathrm{EC} 4 \mathrm{fl} \mathrm{oz}(\mathrm{V} 5)$ & 1.0 & 10.0 & 17.5 & 9.3 \\
\hline 3. Tilt $3.6 \mathrm{EC} 4 \mathrm{fl} \mathrm{oz}(\mathrm{VT} / \mathrm{R} 1)$ & 1.0 & 5.3 & 10.0 & 9.0 \\
\hline 4. Affiance $10 \mathrm{fl} \mathrm{oz}$ (V5) & 1.0 & 7.8 & 17.5 & 9.0 \\
\hline 5. Affiance $10 \mathrm{fl} \mathrm{oz}(\mathrm{VT} / \mathrm{R} 1)$ & 1.0 & 2.0 & 12.5 & 9.5 \\
\hline 6. Headline AMP $10 \mathrm{fl} \mathrm{oz} \mathrm{(V5)}$ & 1.0 & 6.3 & 17.5 & 9.3 \\
\hline 7. Headline AMP $10 \mathrm{fl} \mathrm{oz}(\mathrm{VT} / \mathrm{R} 1)$ & 1.0 & 5.5 & 13.8 & 9.3 \\
\hline 8. Miravis Neo $2.5 \mathrm{SE} 13.7 \mathrm{fl} \mathrm{oz}$ (V5) & 1.0 & 6.5 & 15.3 & 9.8 \\
\hline 9. Miravis Neo $2.5 \mathrm{SE} 13.7 \mathrm{fl} \mathrm{oz}$ (VT/R1) & 1.0 & 6.8 & 10.0 & 9.3 \\
\hline 10. Veltyma $7 \mathrm{fl} \mathrm{oz} \mathrm{(V5)}$ & 1.0 & 6.5 & 15.3 & 9.3 \\
\hline 11. Veltyma $7 \mathrm{fl} \mathrm{oz} \mathrm{(VT/R1)}$ & 1.0 & 4.3 & 8.0 & 9.3 \\
\hline 12. Lucento 4.17 SC $5 \mathrm{fl} \mathrm{oz} \mathrm{(V5)}$ & 1.0 & 8.8 & 17.5 & 9.5 \\
\hline 13. Lucento 4.17 SC $5 \mathrm{fl} \mathrm{oz}$ (VT/R1) & 1.0 & 1.0 & 4.3 & 9.3 \\
\hline 14. Topguard EQ 4.29 SC $7 \mathrm{fl} \mathrm{oz}$ (V5) & 1.0 & 7.8 & 17.5 & 9.0 \\
\hline 15. Topguard EQ 4.29 SC $6 \mathrm{fl} \mathrm{oz} \mathrm{(V7)}$ & 1.0 & 2.0 & 12.5 & 9.3 \\
\hline 16. Topguard EQ 4.29 SC $5 \mathrm{fl} \mathrm{oz} \mathrm{(VT/R1)}$ & 1.0 & 3.0 & 8.8 & 9.8 \\
\hline 17. USF0411 SC $8 \mathrm{fl} \mathrm{oz}$ (V5) & 1.0 & 6.5 & 15.3 & 9.3 \\
\hline 18. USF0411 $8 \mathrm{fl} \mathrm{oz} \mathrm{(VT/R1)}$ & 1.0 & 6.5 & 11.3 & 9.5 \\
\hline 19. Stratego YLD $4 \mathrm{fl} \mathrm{oz} \mathrm{(V5)}$ & 1.0 & 5.3 & 15.0 & 9.0 \\
\hline 20. Stratego YLD $4 \mathrm{fl} \mathrm{oz}$ (VT/R1) & 1.0 & 2.0 & 13.8 & 9.5 \\
\hline$P(\mathrm{~F})$ & 1.0 & 0.09 & 0.13 & 0.93 \\
\hline LSD & -- & N.S. & N.S. & N.S. \\
\hline
\end{tabular}

z Foliar fungicides were applied at V5 (5 leaf collars) on 23 Jun; V7 (7 leaf collars) on 8 Jul; and VT/R1 (tasseling/silking) on 20 Jul. Fungicides were applied with Induce $0.25 \% \mathrm{v} / \mathrm{v}$.

y Percent leaf area with symptoms of foliar disease. 14 Aug rating included overall presence of foliar disease symptoms.

x Plant health rating scale: $1=$ dead plant, $10=$ healthy plant.

Arcsine transformation of percentage data was made in analysis to determine statistical significance. 
Table 2. Effect of fungicide treatment and timing on stay green, plant strength, and yield in corn (CORNFOLFUN120, Suffolk, VA 2020).

\begin{tabular}{|c|c|c|c|c|c|}
\hline $\begin{array}{l}\text { Treatment, } \\
\text { rate/A and timing }\end{array}$ & $\begin{array}{c}\% \text { green }^{y} \\
15 \text { Sep }\end{array}$ & $\begin{array}{c}\text { No. plants } \\
\text { lodged }^{\mathrm{x}} \\
15 \text { Sep } \\
\end{array}$ & $\begin{array}{c}\text { No. } \\
\text { plants/30 } \\
\text { push test }^{\mathrm{w}} \\
15 \mathrm{Sep} \\
\end{array}$ & $\begin{array}{c}\text { Yield } \\
(\mathbf{b u} / \mathbf{A})^{\mathrm{v}}\end{array}$ & $\begin{array}{c}\text { Test weight } \\
(\mathbf{l b} / \mathbf{b u})\end{array}$ \\
\hline 1. Untreated & $6.3 \mathrm{e}$ & 5.5 & 1.3 & 113.1 & 54.0 \\
\hline 2. Tilt $3.6 \mathrm{EC} 4 \mathrm{fl} \mathrm{oz}$ (V5) & $11.3 \mathrm{~b}-\mathrm{e}$ & 4.3 & 0.0 & 88.1 & 53.4 \\
\hline 3. Tilt 3.6 EC $4 \mathrm{fl} \mathrm{oz} \mathrm{(VT/R1)}$ & $21.3 \mathrm{ab}$ & 2.8 & 0.3 & 110.6 & 53.4 \\
\hline 4. Affiance $10 \mathrm{fl} \mathrm{oz}$ (V5) & $12.5 \mathrm{~b}-\mathrm{e}$ & 5.0 & 0.3 & 98.6 & 53.5 \\
\hline 5. Affiance $10 \mathrm{fl} \mathrm{oz}$ (VT/R1) & $16.3 \mathrm{~b}-\mathrm{e}$ & 8.3 & 0.3 & 122.0 & 54.4 \\
\hline 6. Headline AMP $10 \mathrm{fl} \mathrm{oz} \mathrm{(V5)}$ & $16.3 \mathrm{~b}-\mathrm{e}$ & 3.0 & 0.5 & 108.1 & 52.7 \\
\hline 7. Headline AMP $10 \mathrm{fl} \mathrm{oz}$ (VT/R1) & $11.3 \mathrm{~b}-\mathrm{e}$ & 4.0 & 0.8 & 125.1 & 53.8 \\
\hline 8. Miravis Neo 2.5 SE $13.7 \mathrm{fl} \mathrm{oz}$ (V5) & $8.8 \mathrm{de}$ & 7.8 & 1.8 & 119.3 & 53.3 \\
\hline 9. Miravis Neo 2.5 SE $13.7 \mathrm{fl} \mathrm{oz} \mathrm{(VT/R1)}$ & $18.8 \mathrm{~b}-\mathrm{d}$ & 3.0 & 1.3 & 118.8 & 52.7 \\
\hline 10. Veltyma $7 \mathrm{fl} \mathrm{oz} \mathrm{(V5)}$ & $16.3 \mathrm{~b}-\mathrm{e}$ & 4.8 & 0.8 & 118.5 & 53.1 \\
\hline 11. Veltyma $7 \mathrm{fl} \mathrm{oz}(\mathrm{VT} / \mathrm{R} 1)$ & $18.8 \mathrm{~b}-\mathrm{d}$ & 2.8 & 0.5 & 119.6 & 52.6 \\
\hline 12. Lucento $4.17 \mathrm{SC} 5 \mathrm{fl} \mathrm{oz}$ (V5) & $11.3 \mathrm{~b}-\mathrm{e}$ & 9.8 & 0.0 & 115.7 & 52.7 \\
\hline 13. Lucento $4.17 \mathrm{SC} 5 \mathrm{fl} \mathrm{oz}$ (VT/R1) & $20.0 \mathrm{a}-\mathrm{c}$ & 2.8 & 0.3 & 115.2 & 53.0 \\
\hline 14. Topguard EQ 4.29 SC $7 \mathrm{fl} \mathrm{oz} \mathrm{(V5)}$ & $10.0 \mathrm{c}-\mathrm{e}$ & 4.0 & 0.8 & 113.7 & 52.8 \\
\hline 15. Topguard EQ 4.29 SC $6 \mathrm{fl} \mathrm{oz}(\mathrm{V} 7)$ & $17.5 \mathrm{~b}-\mathrm{d}$ & 3.8 & 0.8 & 112.8 & 53.9 \\
\hline 16. Topguard EQ 4.29 SC $5 \mathrm{fl} \mathrm{oz}(\mathrm{VT} / \mathrm{R} 1)$ & $30.0 \mathrm{a}$ & 11.3 & 0.5 & 127.5 & 52.7 \\
\hline 17. USF0411 SC $8 \mathrm{fl} \mathrm{oz} \mathrm{(V5)}$ & $12.5 \mathrm{~b}-\mathrm{e}$ & 7.8 & 1.0 & 117.2 & 53.2 \\
\hline 18. USF0411 $8 \mathrm{fl} \mathrm{oz} \mathrm{(VT/R1)}$ & $17.8 \mathrm{~b}-\mathrm{d}$ & 8.5 & 0.8 & 115.2 & 50.9 \\
\hline 19. Stratego YLD $4 \mathrm{fl} \mathrm{oz} \mathrm{(V5)}$ & $10.0 \mathrm{c}-\mathrm{e}$ & 7.5 & 1.3 & 115.8 & 54.1 \\
\hline 20. Stratego YLD $4 \mathrm{fl} \mathrm{oz}$ (VT/R1) & $13.8 \mathrm{~b}-\mathrm{e}$ & 2.3 & 0.5 & 114.7 & 52.7 \\
\hline$P(\mathrm{~F})$ & 0.02 & 0.79 & 0.32 & 0.72 & 0.33 \\
\hline LSD & 10.79 & N.S. & N.S. & N.S. & N.S. \\
\hline
\end{tabular}

z Foliar fungicides were applied at V5 (5 leaf collars) on 23 Jun; V7 (7 leaf collars) on 8 Jul; and VT/R1 (tasseling/silking) on $20 \mathrm{Jul}$. Fungicides were applied with Induce $0.25 \% \mathrm{v} / \mathrm{v}$.

y Percent green leaf area prior to harvest.

$x$ Mean number of plants lodged in two 30-ft rows.

w Plants observed for push test; data are mean number of stalks per 30 plants/plot that snapped or remained lodged when pushed over.

$\checkmark$ Yields are weight of corn with moisture content of 15.5\%. Corn was hand harvested on 5 Oct. One bushel $=56 \mathrm{lbs}$ of grain. Means in a column followed by the same letter(s) are not significantly different according to Fisher's Protected LSD $(P=0.05)$. Arcsine transformation of percentage data was made in analysis to determine statistical significance. 
TEST ID: CORNFOLFUN220

PURPOSE: To evaluate fungicides and spray timing for control of foliar disease in corn and impact on yield at two locations, site 2

LOCATION: Tidewater AREC, 6321 Holland Rd., Suffolk, VA

CROP INFORMATION:

\begin{tabular}{|l|l|}
\hline Field & $46 \mathrm{~B}$ \\
\hline Crop history & 2019 cotton, 2018 peanut, 2017 sorghum \\
\hline Planting date & 6 May \\
\hline Variety & DKC62-05 \\
\hline Seeding rate & 2 seed/row ft \\
\hline Plot length/width & $30^{\prime} \times 12^{\prime}$ \\
\hline Number of rows & 4 \\
\hline Row spacing & $36^{\prime \prime}$ \\
\hline Alleys (length between blocks) & $8^{\prime}$ \\
\hline Harvest date & $24 \mathrm{Sep}$ \\
\hline
\end{tabular}

EXPERIMENTAL DESIGN: Randomized complete block with four replicates

TREATMENT APPLICATION:

\begin{tabular}{|l|l|}
\hline Equipment & Lee Spider Sprayer \\
\hline Pressure (psi) & $38 \mathrm{psi}$ \\
\hline Nozzle type & Twinjet 8002VS \\
\hline Volume (gal/A) & 19.88 \\
\hline Surfactant & NIS $0.25 \% \mathrm{v} / \mathrm{v}$ \\
\hline
\end{tabular}

\section{TREATMENTS:}

\begin{tabular}{|c|l|c|c|l|}
\hline Trt \# & Fungicide and formulation & Rate, fl oz/A & App. timing & App. date \\
\hline 1 & Untreated control & & & \\
\hline 2 & Tilt 3.6 EC & 4 & V5 & $23 \mathrm{Jun}$ \\
\hline 3 & Tilt 3.6 EC & 4 & VT/R1 & $20 \mathrm{Jul}$ \\
\hline 4 & Affiance & 10 & V5 & $23 \mathrm{Jun}$ \\
\hline 5 & Affiance & 10 & VT/R1 & $20 \mathrm{Jul}$ \\
\hline 6 & Headline AMP & 10 & V5 & $23 \mathrm{Jun}$ \\
\hline 7 & Headline AMP & 10 & VT/R1 & $20 \mathrm{Jul}$ \\
\hline 8 & Miravis Neo 2.5 SE & 13.7 & V5 & $23 \mathrm{Jun}$ \\
\hline 9 & Miravis Neo 2.5 SE & 13.7 & VT/R1 & $20 \mathrm{Jul}$ \\
\hline 10 & Veltyma & 7 & V5 & $23 \mathrm{Jun}$ \\
\hline 11 & Veltyma & 7 & VT/R1 & $20 \mathrm{Jul}$ \\
\hline 12 & Lucento 4.17 SC & 5 & V5 & $23 \mathrm{Jun}$ \\
\hline 13 & Lucento 4.17 SC & 5 & VT/R1 & $20 \mathrm{Jul}$ \\
\hline 14 & Topguard EQ 4.29 SC & 7 & V5 & $23 \mathrm{Jun}$ \\
\hline 15 & Topguard EQ 4.29 SC & 6 & V7 & $8 \mathrm{Jul}$ \\
\hline 16 & Topguard EQ 4.29 SC & 5 & VT/R1 & $20 \mathrm{Jul}$ \\
\hline 17 & USF0411 SC & 8 & V5 & $23 \mathrm{Jun}$ \\
\hline 18 & USF0411 SC & 8 & VT/R1 & $20 \mathrm{Jul}$ \\
\hline 19 & Stratego YLD & 4 & V5 & $23 \mathrm{Jun}$ \\
\hline 20 & Stratego YLD & 4 & VT/R1 & $20 \mathrm{Jul}$ \\
\hline & & & & \\
\hline
\end{tabular}


SOIL TYPE: Nansemond fine sandy loam

Soil fertility report (Jan 2020):

\begin{tabular}{|l|l|l|l|l|l|l|l|l|l|}
\hline $\mathbf{p H}$ & $\mathbf{P}(\mathbf{l b} / \mathbf{A})$ & $\mathbf{K}(\mathbf{l b} / \mathbf{A})$ & $\mathbf{C a}(\mathbf{l b} / \mathbf{A})$ & $\mathbf{M g}(\mathbf{l b} / \mathbf{A})$ & $\mathbf{Z n}(\mathbf{p p m})$ & $\mathbf{M n}(\mathbf{p p m})$ & $\mathbf{C u}(\mathbf{p p m})$ & $\mathbf{F e}(\mathbf{p p m})$ & $\mathbf{B}(\mathbf{p p m})$ \\
\hline 6.0 & 50 & 165 & 621 & 96 & 0.4 & 28 & 0.3 & 32.4 & 0.1 \\
\hline
\end{tabular}

MAINTENANCE CHEMICAL PROGRAMS:

\begin{tabular}{|l|l|}
\hline Fertilizer & Standard \\
\hline Herbicides & Standard \\
\hline Insecticides & Standard \\
\hline Fungicides & None except treatments \\
\hline Nematicides & Standard \\
\hline
\end{tabular}

MAINTENANCE CHEMICAL APPLICATIONS:

\begin{tabular}{|l|l|l|l|}
\hline Date & Type and target & Product and formulation & Rate/A \\
\hline $3 / 26 / 20$ & Herbicide & Roundup WeatherMAX & $1 \mathrm{qt}$ \\
\hline $3 / 26 / 20$ & Herbicide & Liberty 280SL & $1 \mathrm{qt}$ \\
\hline $3 / 26 / 20$ & Herbicide & $2,4-D$ & $1 \mathrm{pt}$ \\
\hline $5 / 13 / 20$ & Herbicide & Bicep II MAGNUM & $2 \mathrm{pt}$ \\
\hline $5 / 13 / 20$ & Herbicide & Simazine 4L & $1 \mathrm{pt}$ \\
\hline $5 / 13 / 20$ & Herbicide & Roundup WeatherMAX & $1 \mathrm{qt}$ \\
\hline $6 / 4 / 20$ & Herbicide & Halex GT & $4 \mathrm{pt}$ \\
\hline $6 / 4 / 20$ & Herbicide & Atrazine & $1.5 \mathrm{pt}$ \\
\hline
\end{tabular}


Table 1. Effect of fungicide treatment and application timing on foliar disease in corn (CORNFOLFUN220, Suffolk, VA 2020).

\begin{tabular}{|c|c|c|c|c|}
\hline \multirow[b]{2}{*}{ Treatment, rate/A and timing ${ }^{\mathrm{z}}$} & \multicolumn{3}{|c|}{$\%$ foliar disease ${ }^{y}$} & \multirow[b]{2}{*}{$\begin{array}{c}\text { Plant health } \\
(1-10)^{x} \\
17 \text { Aug }\end{array}$} \\
\hline & 17 Aug & $\begin{array}{c}\text { Gray leaf spot } \\
5 \text { Sep }\end{array}$ & $\begin{array}{c}\text { Curvularia } \\
\text { leaf spot } \\
5 \text { Sep } \\
\end{array}$ & \\
\hline 1. Untreated & 1.0 & $22.5 \mathrm{a}$ & $25.0 \mathrm{a}$ & 8.0 \\
\hline 2. Tilt $3.6 \mathrm{EC} 4 \mathrm{fl} \mathrm{oz}(\mathrm{V} 5)$ & 1.0 & $18.8 \mathrm{ab}$ & $18.8 \mathrm{a}-\mathrm{d}$ & 8.3 \\
\hline 3. Tilt $3.6 \mathrm{EC} 4 \mathrm{fl} \mathrm{oz}$ (VT/R1) & 1.0 & $10.3 \mathrm{~b}-\mathrm{e}$ & $16.3 \mathrm{a}-\mathrm{d}$ & 7.8 \\
\hline 4. Affiance $10 \mathrm{fl} \mathrm{oz}(\mathrm{V} 5)$ & 1.0 & $20.0 \mathrm{a}$ & $17.5 \mathrm{a}-\mathrm{d}$ & 8.8 \\
\hline 5. Affiance $10 \mathrm{fl} \mathrm{oz}(\mathrm{VT} / \mathrm{R} 1)$ & 1.0 & $9.3 \mathrm{c}-\mathrm{f}$ & $11.5 \mathrm{c}-\mathrm{e}$ & 7.3 \\
\hline 6. Headline AMP $10 \mathrm{fl} \mathrm{oz} \mathrm{(V5)}$ & 1.0 & $13.8 \mathrm{a}-\mathrm{d}$ & $17.5 \mathrm{a}-\mathrm{d}$ & 8.5 \\
\hline 7. Headline AMP $10 \mathrm{fl} \mathrm{oz} \mathrm{(VT/R1)}$ & 1.0 & $7.8 \mathrm{c}-\mathrm{f}$ & $15.0 \mathrm{~b}-\mathrm{d}$ & 8.0 \\
\hline 8. Miravis Neo 2.5 SE $13.7 \mathrm{fl} \mathrm{oz}$ (V5) & 1.0 & $13.8 \mathrm{a}-\mathrm{d}$ & $15.0 \mathrm{~b}-\mathrm{d}$ & 8.5 \\
\hline 9. Miravis Neo $2.5 \mathrm{SE} 13.7 \mathrm{fl} \mathrm{oz}(\mathrm{VT} / \mathrm{R} 1)$ & 1.0 & $1.0 \mathrm{f}$ & $4.3 \mathrm{e}$ & 8.3 \\
\hline 10. Veltyma $7 \mathrm{fl} \mathrm{oz} \mathrm{(V5)}$ & 1.0 & $15.0 \mathrm{a}-\mathrm{c}$ & $16.7 \mathrm{a}-\mathrm{d}$ & 8.3 \\
\hline 11. Veltyma $7 \mathrm{fl} \mathrm{oz}(\mathrm{VT} / \mathrm{R} 1)$ & 1.0 & $1.0 \mathrm{f}$ & $5.3 \mathrm{e}$ & 8.5 \\
\hline 12. Lucento $4.17 \mathrm{SC} 5 \mathrm{fl} \mathrm{oz}$ (V5) & 1.0 & $5.3 \mathrm{~d}-\mathrm{f}$ & $16.7 \mathrm{a}-\mathrm{d}$ & 8.3 \\
\hline 13. Lucento $4.17 \mathrm{SC} 5 \mathrm{fl} \mathrm{oz}(\mathrm{VT} / \mathrm{R} 1)$ & 1.0 & $4.0 \mathrm{ef}$ & $11.3 \mathrm{c}-\mathrm{e}$ & 8.0 \\
\hline 14. Topguard EQ 4.29 SC $7 \mathrm{fl} \mathrm{oz} \mathrm{(V5)}$ & 1.0 & $5.0 \mathrm{e}-\mathrm{f}$ & $12.5 \mathrm{c}-\mathrm{e}$ & 7.3 \\
\hline 15. Topguard EQ 4.29 SC $6 \mathrm{fl} \mathrm{oz} \mathrm{(V7)}$ & 1.0 & $21.7 \mathrm{a}$ & $13.3 \mathrm{c}-\mathrm{e}$ & 6.5 \\
\hline 16. Topguard EQ 4.29 SC $5 \mathrm{fl} \mathrm{oz}(\mathrm{VT} / \mathrm{R} 1)$ & 1.0 & $4.3 \mathrm{ef}$ & $16.3 \mathrm{a}-\mathrm{d}$ & 8.5 \\
\hline 17. USF0411 SC $8 \mathrm{fl} \mathrm{oz} \mathrm{(V5)}$ & 1.0 & $5.0 \mathrm{~d}-\mathrm{f}$ & $20.0 \mathrm{a}-\mathrm{c}$ & 8.0 \\
\hline 18. USF0411 $8 \mathrm{fl} \mathrm{oz} \mathrm{(VT/R1)}$ & 1.0 & $10.3 \mathrm{~b}-\mathrm{e}$ & $10.0 \mathrm{de}$ & 8.3 \\
\hline 19. Stratego YLD $4 \mathrm{fl} \mathrm{oz} \mathrm{(V5)}$ & 1.0 & $16.3 \mathrm{a}-\mathrm{c}$ & $20.0 \mathrm{a}-\mathrm{c}$ & 8.3 \\
\hline 20. Stratego YLD $4 \mathrm{fl} \mathrm{oz} \mathrm{(VT/R1)}$ & 1.0 & $10.0 \mathrm{~b}-\mathrm{e}$ & $22.5 \mathrm{ab}$ & 7.8 \\
\hline$P(\mathrm{~F})$ & 1.0 & 0.0001 & 0.002 & 0.51 \\
\hline LSD & -- & 8.84 & 9.06 & N.S. \\
\hline
\end{tabular}

z Foliar fungicides were applied at V5 (5 leaf collars) on 23 Jun; V7 (7 leaf collars) on 8 Jul; and VT/R1 (tasseling/silking) on 20 Jul. Fungicides were applied with Induce $0.25 \%$ v/v.

y Percent leaf area with symptoms of foliar disease. 17 Aug rating included overall presence of foliar disease symptoms.

x Plant health rating scale: $1=$ dead plant, $10=$ healthy plant.

Means in a column followed by the same letter(s) are not significantly different according to Fisher's Protected LSD ( $P=0.05)$.

Arcsine transformation of percentage data was made in analysis to determine statistical significance. 
Table 2. Effect of fungicide treatment and timing on stay green, plant strength, and yield in corn (CORNFOLFUN220, Suffolk, VA 2020).

\begin{tabular}{|c|c|c|c|c|c|}
\hline $\begin{array}{l}\text { Treatment, } \\
\text { rate/A and timing }\end{array}$ & $\begin{array}{c}\% \text { green }^{y} \\
15 \text { Sep }\end{array}$ & $\begin{array}{c}\text { No. plants } \\
\text { lodged }^{\mathrm{x}} \\
16 \text { Sep } \\
\end{array}$ & $\begin{array}{c}\text { No. } \\
\text { plants/30 } \\
\text { push test }^{\mathrm{w}} \\
16 \mathrm{Sep} \\
\end{array}$ & $\begin{array}{c}\text { Yield } \\
(\mathbf{b u} / \mathbf{A})^{\mathrm{v}}\end{array}$ & $\begin{array}{c}\text { Test weight } \\
(\mathbf{l b} / \mathbf{b u})\end{array}$ \\
\hline 1. Untreated & $1.0 \mathrm{c}$ & 3.8 & $1.3 \mathrm{bc}$ & 163.3 & 53.9 \\
\hline 2. Tilt $3.6 \mathrm{EC} 4 \mathrm{fl} \mathrm{oz}$ (V5) & $1.0 \mathrm{c}$ & 3.5 & $1.0 \mathrm{bc}$ & 141.7 & 54.0 \\
\hline 3. Tilt 3.6 EC $4 \mathrm{fl} \mathrm{oz} \mathrm{(VT/R1)}$ & $2.0 \mathrm{bc}$ & 3.0 & $0.0 \mathrm{c}$ & 136.5 & 53.0 \\
\hline 4. Affiance $10 \mathrm{fl} \mathrm{oz}$ (V5) & $2.0 \mathrm{bc}$ & 0.8 & $0.5 \mathrm{bc}$ & 153.9 & 53.3 \\
\hline 5. Affiance $10 \mathrm{fl} \mathrm{oz}$ (VT/R1) & $4.0 \mathrm{a}$ & 2.3 & $1.3 \mathrm{bc}$ & 103.1 & 53.2 \\
\hline 6. Headline AMP $10 \mathrm{fl} \mathrm{oz}$ (V5) & $1.0 \mathrm{c}$ & 7.3 & $1.0 \mathrm{bc}$ & 138.9 & 54.0 \\
\hline 7. Headline AMP $10 \mathrm{fl} \mathrm{oz}$ (VT/R1) & $1.0 \mathrm{c}$ & 2.5 & $1.8 \mathrm{bc}$ & 138.4 & 53.5 \\
\hline 8. Miravis Neo $2.5 \mathrm{SE} 13.7 \mathrm{fl} \mathrm{oz}(\mathrm{V} 5)$ & $1.0 \mathrm{c}$ & 2.3 & $2.3 \mathrm{ab}$ & 150.8 & 53.6 \\
\hline 9. Miravis Neo $2.5 \mathrm{SE} 13.7 \mathrm{fl} \mathrm{oz}$ (VT/R1) & $3.0 \mathrm{ab}$ & 5.0 & $0.5 \mathrm{bc}$ & 154.4 & 53.5 \\
\hline 10. Veltyma $7 \mathrm{fl} \mathrm{oz} \mathrm{(V5)}$ & $1.0 \mathrm{c}$ & 2.0 & $0.5 \mathrm{bc}$ & 148.9 & 54.4 \\
\hline 11. Veltyma $7 \mathrm{fl} \mathrm{oz}(\mathrm{VT} / \mathrm{R} 1)$ & $4.0 \mathrm{a}$ & 1.3 & $1.8 \mathrm{bc}$ & 144.2 & 53.3 \\
\hline 12. Lucento $4.17 \mathrm{SC} 5 \mathrm{fl} \mathrm{oz}$ (V5) & $2.0 \mathrm{bc}$ & 2.0 & $2.3 \mathrm{ab}$ & 122.8 & 52.5 \\
\hline 13. Lucento 4.17 SC $5 \mathrm{fl} \mathrm{oz}$ (VT/R1) & $3.0 \mathrm{ab}$ & 1.3 & $0.5 \mathrm{bc}$ & 140.6 & 52.1 \\
\hline 14. Topguard EQ 4.29 SC $7 \mathrm{fl} \mathrm{oz} \mathrm{(V5)}$ & $1.0 \mathrm{c}$ & 0.8 & $1.5 \mathrm{bc}$ & 114.1 & 53.1 \\
\hline 15. Topguard EQ 4.29 SC $6 \mathrm{fl} \mathrm{oz}(\mathrm{V} 7)$ & $1.0 \mathrm{c}$ & 1.3 & $4.3 \mathrm{a}$ & 91.6 & 54.0 \\
\hline 16. Topguard EQ 4.29 SC $5 \mathrm{fl} \mathrm{oz} \mathrm{(VT/R1)}$ & $4.0 \mathrm{a}$ & 1.8 & $0.3 \mathrm{bc}$ & 192.2 & 53.7 \\
\hline 17. USF0411 SC $8 \mathrm{fl} \mathrm{oz} \mathrm{(V5)}$ & $1.0 \mathrm{c}$ & 2.8 & $1.3 \mathrm{bc}$ & 103.1 & 53.7 \\
\hline 18. USF0411 $8 \mathrm{fl} \mathrm{oz} \mathrm{(VT/R1)}$ & $1.0 \mathrm{c}$ & 2.0 & $1.5 \mathrm{bc}$ & 147.4 & 54.9 \\
\hline 19. Stratego YLD $4 \mathrm{fl} \mathrm{oz}$ (V5) & $1.0 \mathrm{c}$ & 2.3 & $0.5 \mathrm{bc}$ & 121.0 & 54.1 \\
\hline 20. Stratego YLD $4 \mathrm{fl} \mathrm{oz}$ (VT/R1) & $3.0 \mathrm{ab}$ & 2.8 & $0.3 \mathrm{bc}$ & 151.3 & 53.0 \\
\hline$P(\mathrm{~F})$ & 0.001 & 0.64 & 0.05 & 0.72 & 0.34 \\
\hline LSD & 1.93 & N.S. & 2.08 & N.S. & N.S. \\
\hline
\end{tabular}

z Foliar fungicides were applied at V5 (5 leaf collars) on 23 Jun; V7 (7 leaf collars) on 8 Jul; and VT/R1 (tasseling/silking) on 20 Jul. Fungicides were applied with Induce $0.25 \% \mathrm{v} / \mathrm{v}$.

y Percent green leaf area prior to harvest.

$x$ Mean number of plants lodged in two 30-ft rows.

w Plants observed for push test; data are mean number of stalks per 30 plants/plot that snapped or remained lodged when pushed over.

v Yields are weight of corn with moisture content of 15.5\%. Corn was harvested on 24 Sep. One bushel $=56$ lbs of grain. Means in a column followed by the same letter(s) are not significantly different according to Fisher's Protected LSD $(P=0.05)$. Arcsine transformation of percentage data was made in analysis to determine statistical significance. 
TEST ID: PTSSR120

PURPOSE: To evaluate fungicide treatments for control of southern stem rot in peanut and impact on yield

LOCATION: Tidewater AREC, 6321 Holland Rd., Suffolk, VA

CROP INFORMATION:

\begin{tabular}{|l|l|}
\hline Field & $46 \mathrm{~A}$ \\
\hline Crop history & 2019 corn, 2018 cotton, 2017 peanut \\
\hline Planting date & 15 May \\
\hline Variety & Sullivan \\
\hline Seeding rate & ca. 4 seed/row ft $(143 \mathrm{lb} / \mathrm{A})$ \\
\hline Plot length/width & $35^{\prime} \times 12^{\prime}$ \\
\hline Number of rows & 4 \\
\hline Row spacing & $36^{\prime}$ \\
\hline Alleys (length between blocks) & $8^{\prime}$ \\
\hline Harvest date & 9 Nov \\
\hline
\end{tabular}

EXPERIMENTAL DESIGN: Randomized complete block with four replicates

TREATMENT APPLICATION:

\begin{tabular}{|l|l|}
\hline & Foliar spray \\
\hline Equipment & Tractor plot sprayer \\
\hline Pressure (psi) & $38 \mathrm{psi}$ \\
\hline Nozzle type & $8002 \mathrm{TwinJet}$ \\
\hline Volume $(\mathbf{g a l} / \mathbf{A})$ & $19.88 \mathrm{gal} / \mathrm{A}$ \\
\hline
\end{tabular}

\section{APPLICATION SCHEDULE:}

\begin{tabular}{|l|l|}
\hline A & $R 3$ \\
\hline B & $\mathrm{A}+14 \mathrm{~d}$ \\
\hline $\mathbf{C}$ & $\mathrm{A}+28 \mathrm{~d}$ \\
\hline $\mathbf{D}$ & $\mathrm{A}+42 \mathrm{~d}$ \\
\hline E & $\mathrm{A}+56 \mathrm{~d}$ \\
\hline
\end{tabular}

\section{TREATMENTS:}

\begin{tabular}{|l|l|l|l|l|}
\hline Trt \# & Product and formulation & Rate/A & Appl. timing & Appl. date \\
\hline 1 & Bravo Weather Stik & $24 \mathrm{fl} \mathrm{oz}$ & ABCDE & 17 Jul, 30 Jul, 13 Aug, 27 Aug, 16 Sep \\
\hline 2 & Bravo Weather Stik & $24 \mathrm{fl} \mathrm{oz}$ & ACE & 17 Jul, 13 Aug, 16 Sep \\
& Elatus 45WG & $9.5 \mathrm{oz} \mathrm{wt}$ & BD & 30 Jul, 27 Aug \\
\hline 3 & Bravo Weather Stik & $24 \mathrm{fl} \mathrm{oz}$ & ACE & 17 Jul, 13 Aug, 16 Sep \\
& Convoy SC & $16 \mathrm{fl} \mathrm{oz}$ & BD & 30 Jul, 27 Aug \\
\hline 4 & Bravo Weather Stik & $24 \mathrm{fl} \mathrm{oz}$ & ABCDE & 17 Jul, 13 Aug, 16 Sep \\
& Excalia SC & $2 \mathrm{fl} \mathrm{oz}$ & BD & 30 Jul, 27 Aug \\
\hline 5 & Bravo Weather Stik & $24 \mathrm{fl} \mathrm{oz}$ & ABCDE & 17 Jul, 13 Aug, 16 Sep \\
& Excalia SC & $3 \mathrm{fl} \mathrm{oz}$ & BD & 30 Jul, 27 Aug \\
\hline 6 & Bravo Weather Stik & $24 \mathrm{fl} \mathrm{oz}$ & ABCDE & 17 Jul, 13 Aug, 16 Sep \\
& Excalia SC & $4 \mathrm{fl} \mathrm{oz}$ & BD & 30 Jul, 27 Aug \\
\hline
\end{tabular}


SOIL TYPE: Nansemond fine sandy loam

SOIL FERTILITY REPORT (Jan 2020):

\begin{tabular}{|l|l|l|l|l|l|l|l|l|l|}
\hline $\mathbf{p H}$ & $\mathbf{P}(\mathbf{l b} / \mathbf{A})$ & $\mathbf{K}(\mathbf{l b} / \mathbf{A})$ & $\mathbf{C a}(\mathbf{l b} / \mathbf{A})$ & $\mathbf{M g}(\mathbf{l b} / \mathbf{A})$ & $\mathbf{Z n}(\mathbf{p p m})$ & $\mathbf{M n}(\mathbf{p p m})$ & $\mathbf{C u}(\mathbf{p p m})$ & $\mathbf{F e}(\mathbf{p p m})$ & $\mathbf{B}(\mathbf{p p m})$ \\
\hline 6.1 & 95 & 164 & 858 & 77 & 0.7 & 3.1 & 0.4 & 40.5 & 0.1 \\
\hline
\end{tabular}

MAINTENANCE CHEMICAL PROGRAMS:

\begin{tabular}{|l|l|}
\hline Fertilizer & Standard \\
\hline Herbicides & Standard \\
\hline Insecticides & Admire Pro IF, standard \\
\hline Fungicides & Proline IF \\
\hline Nematicides & None \\
\hline
\end{tabular}

MAINTENANCE CHEMICAL APPLICATIONS:

\begin{tabular}{|l|l|l|l|}
\hline Date & Type and target & Product and formulation & Rate/A \\
\hline $5 / 15 / 20$ & Insecticide & Admire Pro 4.6 SC & $8.5 \mathrm{fl} \mathrm{oz}$ \\
\hline $5 / 15 / 20$ & Fungicide & Proline 480 SC & $5.7 \mathrm{fl} \mathrm{oz}$ \\
\hline $5 / 17 / 20$ & Herbicide & Strongarm & $0.45 \mathrm{fl} \mathrm{oz}$ \\
\hline $5 / 17 / 20$ & Herbicide & Dual II MAGNUM & $1.5 \mathrm{pt}$ \\
\hline $5 / 17 / 20$ & Herbicide & Prowl H2O & $1.0 \mathrm{pt}$ \\
\hline $5 / 17 / 20$ & Fertility & Boron & $1.0 \mathrm{qt}$ \\
\hline $6 / 4 / 20$ & Insecticide & Orthene 75 S & $12 \mathrm{oz}$ \\
\hline $6 / 25 / 20$ & Herbicide & Basagran & $1.5 \mathrm{pt}$ \\
\hline $6 / 25 / 20$ & Herbicide & Fusilade DX & $1 \mathrm{pt}$ \\
\hline $7 / 7 / 20$ & Herbicide & Storm & $1.5 \mathrm{pt}$ \\
\hline $7 / 7 / 20$ & Herbicide & Basagran & $1 \mathrm{pt}$ \\
\hline $7 / 13 / 20$ & Fertility & Manganese & $2 \mathrm{qt}$ \\
\hline $7 / 13 / 20$ & Insecticide & Danitol 2.4 EC & $8 \mathrm{fl} \mathrm{oz}$ \\
\hline $7 / 16 / 20$ & Fertility & Landplaster & $1500 \mathrm{lb}$ \\
\hline $7 / 24 / 20$ & Insecticide & Danitol 2.4 EC & $8 \mathrm{fl} \mathrm{oz}$ \\
\hline $8 / 6 / 20$ & Herbicide & Tide 2 EC (Clethodim) & $16 \mathrm{fl} \mathrm{oz}$ \\
\hline & & & \\
\hline
\end{tabular}


Table 1. Effect of fungicide treatment on soilborne disease in peanut (PTSSR120, Suffolk, VA 2020).

\begin{tabular}{|c|c|c|c|c|c|c|}
\hline \multirow{2}{*}{$\begin{array}{l}\text { Treatment, rate/A } \\
\text { and application date }{ }^{\mathrm{z}}\end{array}$} & \multirow{2}{*}{$\begin{array}{c}\begin{array}{c}\text { Southern } \\
\text { stem rot }^{y}\end{array} \\
13 \text { Sep } \\
\end{array}$} & \multicolumn{5}{|c|}{ Sclerotinia blight $^{\mathbf{y}}$} \\
\hline & & 28 Aug & 13 Sep & 27 Sep & 17 Oct & AUDPC $^{x}$ \\
\hline $\begin{array}{ll}\text { 1. } & \text { Bravo Weather Stik } 24 \mathrm{fl} \mathrm{oz} \\
& (7 / 17,7 / 30,8 / 13,8 / 27,9 / 16) \\
\end{array}$ & 0.0 & 2.8 & 9.0 & 16.5 & 31.0 & 747.5 \\
\hline $\begin{array}{l}\text { 2. Bravo Weather Stik } 24 \mathrm{fl} \mathrm{oz}(7 / 17,8 / 13,9 / 16) \\
\text { Elatus WG } 9.5 \mathrm{oz} \text { wt }(7 / 30,8 / 27)\end{array}$ & 0.0 & 0.8 & 4.5 & 12.5 & 20.8 & 493.5 \\
\hline $\begin{array}{l}\text { 3. Bravo Weather Stik } 24 \mathrm{fl} \mathrm{oz}(7 / 17,8 / 13,9 / 16) \\
\text { Convoy SC } 16 \mathrm{fl} \mathrm{oz}(7 / 30,8 / 27)\end{array}$ & 0.0 & 2.0 & 9.0 & 19.5 & 28.0 & 762.5 \\
\hline $\begin{array}{l}\text { 4. Bravo Weather Stik } 24 \mathrm{fl} \mathrm{oz}(7 / 17,8 / 13,9 / 16) \\
\text { Excalia SC } 2 \mathrm{fl} \mathrm{oz}(7 / 30,8 / 27)\end{array}$ & 0.0 & 1.5 & 7.0 & 15.0 & 25.8 & 629.5 \\
\hline $\begin{array}{l}\text { 5. Bravo Weather Stik } 24 \mathrm{fl} \mathrm{oz}(7 / 17,8 / 13,9 / 16) \\
\text { Excalia SC } 3 \mathrm{fl} \mathrm{oz}(7 / 30,8 / 27)\end{array}$ & 0.0 & 0.3 & 6.3 & 17.5 & 27.5 & 668.3 \\
\hline $\begin{array}{l}\text { 6. Bravo Weather Stik } 24 \mathrm{fl} \mathrm{oz}(7 / 17,8 / 13,9 / 16) \\
\text { Excalia SC } 4 \mathrm{fl} \mathrm{oz}(7 / 30,8 / 27)\end{array}$ & 0.3 & 1.0 & 3.8 & 15.3 & 27.5 & 598.5 \\
\hline$P(\mathrm{~F})$ & 0.45 & 0.55 & 0.30 & 0.63 & 0.27 & 0.47 \\
\hline LSD & N.S. & N.S. & N.S. & N.S. & N.S. & N.S. \\
\hline
\end{tabular}

z Treatments were applied on a calendar-based schedule beginning at R3 (17 Jul) and followed by two- or four-week intervals between sprays when prescribed. Seed was planted 15 May.

$y$ Counts of infection centers in the two center rows of each plot or a total of $70 \mathrm{ft}$ row. An infection center was a point with symptoms and/or signs of a disease and included $6 \mathrm{in}$. on either side of that point. No incidences of stem rot were found on observations on 28 Aug, 14 Sep, 27 Sep, and 17 Oct.

$x \quad$ AUDPC $=$ area under the disease progress curve.

Table 2. Effect of fungicide treatment on disease incidence and yield in peanut (PTSSR120, Suffolk, VA 2020).

\begin{tabular}{|c|c|c|}
\hline $\begin{array}{l}\text { Treatment, rate/A } \\
\text { and application date } \\
\end{array}$ & $\begin{array}{l}\text { \% leaf spot } \text { ly }^{\mathbf{y}} \\
\text { 9 Oct }\end{array}$ & $\begin{array}{l}\text { Yield }^{\mathrm{x}} \\
(\mathbf{l b} / \mathrm{A})\end{array}$ \\
\hline 1. Bravo Weather Stik $24 \mathrm{fl} \mathrm{oz}(7 / 17,7 / 30,8 / 13,8 / 27,9 / 16)$ & $1.0 \mathrm{~b}$ & 2142 \\
\hline $\begin{array}{ll}\text { 2. } & \text { Bravo Weather Stik } 24 \mathrm{fl} \mathrm{oz}(7 / 17,8 / 13,9 / 16) \\
\text { Elatus WG } 9.5 \mathrm{oz} \text { wt }(7 / 30,8 / 27)\end{array}$ & $1.0 \mathrm{~b}$ & 3011 \\
\hline $\begin{array}{l}\text { 3. Bravo Weather Stik } 24 \mathrm{fl} \mathrm{oz}(7 / 17,8 / 13,9 / 16) \\
\text { Convoy SC } 16 \mathrm{fl} \mathrm{oz}(7 / 30,8 / 27)\end{array}$ & $5.3 \mathrm{a}$ & 2399 \\
\hline $\begin{array}{l}\text { 4. Bravo Weather Stik } 24 \mathrm{fl} \mathrm{oz}(7 / 17,8 / 13,9 / 16) \\
\text { Excalia SC } 2 \mathrm{fl} \mathrm{oz}(7 / 30,8 / 27)\end{array}$ & $1.0 \mathrm{~b}$ & 2552 \\
\hline $\begin{array}{l}\text { 5. Bravo Weather Stik } 24 \mathrm{fl} \mathrm{oz}(7 / 17,8 / 13,9 / 16) \\
\text { Excalia SC } 3 \mathrm{fl} \mathrm{oz}(7 / 30,8 / 27)\end{array}$ & $1.0 \mathrm{~b}$ & 2370 \\
\hline $\begin{array}{l}\text { 6. Bravo Weather Stik } 24 \mathrm{fl} \mathrm{oz}(7 / 17,8 / 13,9 / 16) \\
\text { Excalia SC } 4 \mathrm{fl} \mathrm{oz}(7 / 30,8 / 27)\end{array}$ & $2.0 \mathrm{~b}$ & 2611 \\
\hline$P(\mathrm{~F})$ & $\mathbf{0 . 0 3}$ & 0.43 \\
\hline LSD & 2.74 & N.S. \\
\hline
\end{tabular}

z Treatments were applied on a calendar-based schedule beginning at R3 (17 Jul) and followed by two- or four-week intervals between sprays when prescribed. Seed was planted 15 May.

$y$ Percent of leaves in two treatment rows with symptoms of leaf spot.

x Yields are weight of peanuts with moisture content adjusted to 7\%. Peanuts were dug 20 Oct and harvested 9 Nov. Means in a column followed by the same letter(s) are not significantly different according to Fisher's Protected LSD $(P=0.05)$. Arcsine transformation of percentage data was made in analysis to determine statistical significance. 
TEST ID: PTSCL120

PURPOSE: To evaluate fungicide treatments for control of Sclerotinia blight in peanut and impact on yield

LOCATION: Tidewater AREC, 6321 Holland Rd., Suffolk, VA

CROP INFORMATION:

\begin{tabular}{|l|l|}
\hline Field & $46 \mathrm{~A}$ \\
\hline Crop history & 2019 corn, 2018 cotton, 2017 peanut \\
\hline Planting date & 15 May \\
\hline Variety & Sullivan \\
\hline Seeding rate & ca. 4 seed/row ft $(143 \mathrm{lb} / \mathrm{A})$ \\
\hline Plot length/width & $35^{\prime} \times 12^{\prime}$ \\
\hline Number of rows & 4 \\
\hline Row spacing & $36^{\prime}$ \\
\hline Alleys (length between blocks) & $8^{\prime}$ \\
\hline Harvest date & 9 Nov \\
\hline
\end{tabular}

EXPERIMENTAL DESIGN: Randomized complete block with four replicates

TREATMENT APPLICATION:

\begin{tabular}{|l|l|}
\hline & Foliar spray \\
\hline Equipment & Tractor plot sprayer \\
\hline Pressure (psi) & $38 \mathrm{psi}$ \\
\hline Nozzle type & $8002 \mathrm{TwinJet}$ \\
\hline Volume (gal/A) & $19.88 \mathrm{gal} / \mathrm{A}$ \\
\hline
\end{tabular}

\section{APPLICATION SCHEDULE:}

\begin{tabular}{|l|l|}
\hline A & Advisory Spray \\
\hline B & A $+21 d$ \\
\hline C & $A+28 d$ \\
\hline D & $A+42 d$ \\
\hline E & $A+56 d$ \\
\hline F & $A+63 d$ \\
\hline
\end{tabular}

TREATMENTS:

\begin{tabular}{|l|l|l|l|l|}
\hline Trt \# & Product and formulation & Rate/A & Appl. timing & Appl. date \\
\hline 1 & Untreated & & & \\
\hline 2 & Omega 500 F & $16 \mathrm{fl} \mathrm{oz}$ & ABD & 27 Jul, 18 Aug, 14 Sep \\
\hline 3 & Omega 500 F & $24 \mathrm{fl} \mathrm{oz}$ & ACE & 27 Jul, 27 Aug, 24 Sep \\
\hline 4 & Excalia SC & $2 \mathrm{fl} \mathrm{oz}$ & ABD & 27 Jul, 18 Aug, 14 Sep \\
\hline 5 & Excalia SC & $4 \mathrm{fl} \mathrm{oz}$ & ACE & 27 Jul, 27 Aug, 24 Sep \\
\hline 6 & Omega 500 F & $24 \mathrm{fl} \mathrm{oz}$ & AD & 27 Jul, 14 Sep \\
& Excalia SC & $4 \mathrm{fl} \mathrm{oz}$ & BF & 18 Aug, 5 Oct \\
\hline 7 & Pyraziflumid 20 SC & $4.66 \mathrm{fl} \mathrm{oz}$ & ABD & 27 Jul, 18 Aug, 14 Sep \\
\hline 8 & Pyraziflumid 20 SC & $9.33 \mathrm{fl} \mathrm{oz}$ & ABD & 27 Jul, 18 Aug, 14 Sep \\
\hline
\end{tabular}


SOIL TYPE: Nansemond fine sandy loam

SOIL FERTILITY REPORT (Jan 2020):

\begin{tabular}{|l|l|l|l|l|l|l|l|l|l|}
\hline $\mathbf{p H}$ & $\mathbf{P}(\mathbf{l b} / \mathbf{A})$ & $\mathbf{K}(\mathbf{l b} / \mathbf{A})$ & $\mathbf{C a}(\mathbf{l b} / \mathbf{A})$ & $\mathbf{M g}(\mathbf{l b} / \mathbf{A})$ & $\mathbf{Z n}(\mathbf{p p m})$ & $\mathbf{M n}(\mathbf{p p m})$ & $\mathbf{C u}(\mathbf{p p m})$ & $\mathbf{F e}(\mathbf{p p m})$ & $\mathbf{B}(\mathbf{p p m})$ \\
\hline 6.1 & 95 & 164 & 858 & 77 & 0.7 & 3.1 & 0.4 & 40.5 & 0.1 \\
\hline
\end{tabular}

MAINTENANCE CHEMICAL PROGRAMS:

\begin{tabular}{|l|l|}
\hline Fertilizer & Standard \\
\hline Herbicides & Standard \\
\hline Insecticides & Admire Pro IF standard \\
\hline Fungicides & Proline IF \\
\hline Nematicides & None \\
\hline
\end{tabular}

MAINTENANCE CHEMICAL APPLICATIONS:

\begin{tabular}{|l|l|l|l|}
\hline Date & Type and target & Product and formulation & Rate/A \\
\hline $5 / 15 / 20$ & Insecticide & Admire Pro 4.6 SC & $8.5 \mathrm{fl} \mathrm{oz}$ \\
\hline $5 / 15 / 20$ & Fungicide & Proline 480 SC & $5.7 \mathrm{fl} \mathrm{oz}$ \\
\hline $5 / 17 / 20$ & Herbicide & Strongarm & $0.45 \mathrm{fl} \mathrm{oz}$ \\
\hline $5 / 17 / 20$ & Herbicide & Dual II MAGNUM & $1.5 \mathrm{pt}$ \\
\hline $5 / 17 / 20$ & Herbicide & Prowl H2O & $1.0 \mathrm{pt}$ \\
\hline $5 / 17 / 20$ & Fertility & Boron & $1.0 \mathrm{qt}$ \\
\hline $6 / 4 / 20$ & Insecticide & Orthene 75S & $12 \mathrm{oz}$ \\
\hline $6 / 25 / 20$ & Herbicide & Basagran & $1.5 \mathrm{pt}$ \\
\hline $6 / 25 / 20$ & Herbicide & Fusilade DX & $1 \mathrm{pt}$ \\
\hline $7 / 7 / 20$ & Herbicide & Storm & $1.5 \mathrm{pt}$ \\
\hline $7 / 7 / 20$ & Herbicide & Basagran & $1 \mathrm{pt}$ \\
\hline $7 / 13 / 20$ & Fungicide & Bravo Weather Stik & $1.5 \mathrm{pt}$ \\
\hline $7 / 13 / 20$ & Fertility & Manganese & $2 \mathrm{qt}$ \\
\hline $7 / 13 / 20$ & Insecticide & Danitol 2.4 EC & $8 \mathrm{fl} \mathrm{oz}$ \\
\hline $7 / 16 / 20$ & Fertility & Landplaster & $1500 \mathrm{lb}$ \\
\hline $7 / 24 / 20$ & Insecticide & Danitol 2.4 EC & $8 \mathrm{fl} \mathrm{oz}$ \\
\hline $8 / 6 / 20$ & Herbicide & Tide 2 EC (Clethodim) & $16 \mathrm{fl} \mathrm{oz}$ \\
\hline $8 / 10 / 20$ & Fungicide & Bravo Weather Stik & $1.5 \mathrm{pt}$ \\
\hline $8 / 24 / 20$ & Fungicide & Bravo Weather Stik & $1.5 \mathrm{pt}$ \\
\hline & & &
\end{tabular}


Table 1. Effect of fungicide treatment on soilborne disease incidence in peanut (PTSCL120, Suffolk, VA 2020).

\begin{tabular}{|c|c|c|c|c|c|}
\hline \multirow[b]{2}{*}{ Treatment, rate/A and application date ${ }^{\mathrm{z}}$} & \multicolumn{5}{|c|}{ Sclerotinia blight $^{\mathbf{y}}$} \\
\hline & 28 Aug & 13 Sep & 27 Sep & 17 Oct & AUDPC $^{x}$ \\
\hline 1. Untreated & 1.8 & $10.8 \mathrm{a}$ & $21.3 \mathrm{a}$ & $29.3 \mathrm{a}$ & $829.0 \mathrm{a}$ \\
\hline 2. Omega $500 \mathrm{~F} 16 \mathrm{fl} \mathrm{oz}(7 / 27,8 / 18,9 / 14)$ & 0.3 & $1.5 \mathrm{bc}$ & $2.5 \mathrm{~cd}$ & $7.5 \mathrm{~b}$ & $142.0 \mathrm{c}$ \\
\hline 3. Omega $500 \mathrm{~F} 24 \mathrm{fl} \mathrm{oz}(7 / 27,8 / 27,9 / 24)$ & 0.5 & $2.0 \mathrm{bc}$ & $4.3 \mathrm{~cd}$ & $8.0 \mathrm{~b}$ & $186.3 \mathrm{bc}$ \\
\hline 4. Excalia SC $2 \mathrm{fl} \mathrm{oz}(7 / 27,8 / 18,9 / 14)$ & 0.0 & $4.8 \mathrm{bc}$ & $11.0 \mathrm{bc}$ & $25.3 \mathrm{a}$ & $510.8 \mathrm{ab}$ \\
\hline 5. Excalia SC $4 \mathrm{fl} \mathrm{oz}(7 / 27,8 / 27,9 / 24)$ & 1.0 & $5.5 \mathrm{ab}$ & $15.5 \mathrm{ab}$ & $24.8 \mathrm{a}$ & $601.5 \mathrm{a}$ \\
\hline $\begin{array}{l}\text { 6. Omega } 500 \mathrm{~F} 24 \mathrm{fl} \mathrm{oz}(7 / 27,9 / 14) \\
\text { Excalia SC } 4 \mathrm{fl} \mathrm{oz}(8 / 18,(5 \mathrm{Oct}))\end{array}$ & 0.0 & $1.8 \mathrm{bc}$ & $4.0 \mathrm{~cd}$ & $7.5 \mathrm{~b}$ & $169.3 \mathrm{c}$ \\
\hline 7. Pyraziflumid $20 \mathrm{SC} 4.66 \mathrm{fl} \mathrm{oz}(7 / 27,8 / 18,9 / 14)$ & 0.0 & $0.5 \mathrm{bc}$ & $1.0 \mathrm{~d}$ & $4.3 \mathrm{~b}$ & $67.0 \mathrm{c}$ \\
\hline 8. Pyraziflumid 20 SC $9.33 \mathrm{fl} \mathrm{oz}(7 / 27,8 / 18,9 / 14)$ & 0.0 & $0.0 \mathrm{c}$ & $0.3 \mathrm{~d}$ & $0.5 \mathrm{~b}$ & $9.3 \mathrm{c}$ \\
\hline$P(\mathrm{~F})$ & 0.13 & 0.009 & 0.001 & $<0.0001$ & $\mathbf{0 . 0 0 0 2}$ \\
\hline LSD & N.S. & 5.42 & 9.33 & 8.99 & 328.52 \\
\hline
\end{tabular}

$\mathrm{z}$ First fungicide application for Sclerotinia blight was applied according to the Virginia Sclerotinia Blight Advisory (27 Jul).

y Counts of infection centers in the two center rows of each plot or a total of $70 \mathrm{ft}$ row. An infection center was a point with symptoms and/or signs of a disease and included 6 in. on either side of that point.

$x \quad$ AUDPC $=$ area under the disease progress curve.

Means in a column followed by the same letter(s) are not significantly different according to Fisher's Protected LSD $(P=0.05)$.

Table 2. Effect of fungicide treatment on disease incidence and yield in peanut (PTSCL120, Suffolk, VA 2020).

\begin{tabular}{|c|c|c|}
\hline Treatment, rate/A and application date ${ }^{\mathrm{z}}$ & $\begin{array}{l}\text { \% leaf spot }{ }^{\mathrm{y}} \\
\text { 9 Oct }\end{array}$ & $\begin{array}{l}\text { Yield }^{\mathrm{x}} \\
(\mathrm{lb} / \mathrm{A})\end{array}$ \\
\hline 1. Untreated & 3.0 & $2286 \mathrm{c}$ \\
\hline 2. Omega $500 \mathrm{~F} 16 \mathrm{fl} \mathrm{oz}(7 / 27,8 / 18,9 / 14)$ & 1.0 & $3536 \mathrm{~b}$ \\
\hline 3. Omega $500 \mathrm{~F} 24 \mathrm{fl} \mathrm{oz}(7 / 27,8 / 27,9 / 24)$ & 1.0 & $3522 \mathrm{~b}$ \\
\hline 4. Excalia SC $2 \mathrm{fl} \mathrm{oz}(7 / 27,8 / 18,9 / 14)$ & 2.0 & $2466 \mathrm{c}$ \\
\hline 5. Excalia SC $4 \mathrm{fl} \mathrm{oz}(7 / 27,8 / 27,9 / 24)$ & 1.0 & $3172 \mathrm{bc}$ \\
\hline $\begin{array}{l}\text { 6. Omega } 500 \mathrm{~F} 24 \mathrm{fl} \mathrm{oz}(7 / 27,9 / 14) \\
\text { Excalia SC } 4 \mathrm{fl} \mathrm{oz}(8 / 18,(5 \mathrm{Oct}))\end{array}$ & 1.0 & $3776 \mathrm{ab}$ \\
\hline 7. Pyraziflumid $20 \mathrm{SC} 4.66 \mathrm{fl} \mathrm{oz}(7 / 27,8 / 18,9 / 14)$ & 1.0 & $4097 \mathrm{ab}$ \\
\hline 8. Pyraziflumid $20 \mathrm{SC} 9.33 \mathrm{fl} \mathrm{oz}(7 / 27,8 / 18,9 / 14)$ & 1.0 & $4713 \mathrm{a}$ \\
\hline$P(\mathrm{~F})$ & 0.09 & 0.001 \\
\hline LSD & N.S. & 954.6 \\
\hline
\end{tabular}

z First fungicide application for Sclerotinia blight was applied according to the Virginia Sclerotinia Blight Advisory (27 Jul).

y Percent leaves with symptoms of late leaf spot.

x Yields are weight of peanuts with moisture content adjusted to 7\%. Peanuts were dug 20 Oct and harvested 9 Nov.

Means in a column followed by the same letter(s) are not significantly different according to Fisher's Protected LSD

$(P=0.05)$. Arcsine transformation of percentage data was made in analysis to determine statistical significance. 
TEST ID: PTLFSPOT120

PURPOSE: To evaluate fungicide treatments for control of peanut leaf spot and Sclerotinia blight, and impact on yield

LOCATION: Tidewater Research Farm, Hare Rd., Suffolk, VA

CROP INFORMATION:

\begin{tabular}{|l|l|}
\hline Field & 34B \\
\hline Crop history & 2019 corn, 2018 cotton, 2017 peanut \\
\hline Planting date & 16 May \\
\hline Variety & Sullivan \\
\hline Seeding rate & ca. 4 seed/row ft $(143 \mathrm{lb} / \mathrm{A})$ \\
\hline Plot length/width & $35^{\prime} \times 12^{\prime}$ \\
\hline Number of rows & 4 \\
\hline Row spacing & $366^{\prime}$ \\
\hline Alleys (length between blocks) & $8^{\prime}$ \\
\hline Harvest date & 4 Nov \\
\hline
\end{tabular}

EXPERIMENTAL DESIGN: Randomized complete block with four replicates

\section{TREATMENT APPLICATION:}

\begin{tabular}{|l|l|}
\hline & Foliar spray \\
\hline Equipment & Tractor plot sprayer \\
\hline Pressure (psi) & $38 \mathrm{psi}$ \\
\hline Nozzle type & $8002 \mathrm{TwinJet}$ \\
\hline Volume $($ gal/A) & $19.88 \mathrm{gal} / \mathrm{A}$ \\
\hline Surfactant & NIS $0.125 \% \mathrm{v} / \mathrm{v}$ \\
\hline
\end{tabular}

\section{APPLICATION SCHEDULE:}

\begin{tabular}{|l|l|}
\hline A & 35 DAP \\
\hline B & 49 DAP \\
\hline C & 56 DAP \\
\hline D & 63 DAP \\
\hline E & 77 DAP \\
\hline F & 84 DAP \\
\hline G & 91 DAP \\
\hline H & 112 DAP \\
\hline I & 119 DAP \\
\hline J & 126 DAP \\
\hline
\end{tabular}


TREATMENTS:

\begin{tabular}{|c|c|c|c|c|}
\hline Trt \# & Product and formulation & Rate/A & Appl. timing & Appl. date \\
\hline 1 & Untreated control & & & \\
\hline 2 & $\begin{array}{l}\text { Provysol SC } \\
\text { Bravo Weather Stik } 6 \text { SC }\end{array}$ & $\begin{array}{l}5 \mathrm{fl} \mathrm{oz} \\
24 \mathrm{fl} \mathrm{oz} \\
\end{array}$ & $\begin{array}{l}\text { ACGH } \\
\mathrm{E}\end{array}$ & $\begin{array}{l}22 \text { Jun, } 10 \text { Jul, } 13 \text { Aug, } 8 \text { Sep } \\
31 \text { Jul }\end{array}$ \\
\hline 3 & $\begin{array}{l}\text { Provost Silver } 3.52 \text { SC } \\
\text { Bravo Weather Stik 6 SC }\end{array}$ & $\begin{array}{l}12.8 \mathrm{fl} \mathrm{oz} \\
24 \mathrm{fl} \mathrm{oz}\end{array}$ & $\begin{array}{l}\text { ACGH } \\
\text { E }\end{array}$ & $\begin{array}{l}22 \text { Jun, } 10 \text { Jul, } 13 \text { Aug, } 8 \text { Sep } \\
\text { 31 Jul }\end{array}$ \\
\hline 4 & $\begin{array}{l}\text { Inspire EC } \\
\text { Bravo Weather Stik } 6 \text { SC }\end{array}$ & $\begin{array}{l}7 \mathrm{fl} \mathrm{oz} \\
24 \mathrm{fl} \mathrm{oz}\end{array}$ & $\begin{array}{l}\text { ACGH } \\
\text { E }\end{array}$ & $\begin{array}{l}22 \text { Jun, } 10 \text { Jul, } 13 \text { Aug, } 8 \text { Sep } \\
\text { 31 Jul }\end{array}$ \\
\hline 5 & $\begin{array}{l}\text { Alto } 100 \text { SL } \\
\text { Bravo Weather Stik } 6 \text { SC }\end{array}$ & $\begin{array}{l}5.5 \mathrm{fl} \mathrm{oz} \\
24 \mathrm{fl} \mathrm{oz} \\
\end{array}$ & $\begin{array}{l}\text { ACGH } \\
\mathrm{E}\end{array}$ & $\begin{array}{l}22 \text { Jun, } 10 \text { Jul, } 13 \text { Aug, } 8 \text { Sep } \\
31 \text { Jul }\end{array}$ \\
\hline 6 & $\begin{array}{l}\text { Bravo Top SC } \\
\text { Bravo Weather Stik } 6 \text { SC }\end{array}$ & $\begin{array}{l}34 \mathrm{fl} \mathrm{oz} \\
24 \mathrm{fl} \mathrm{oz}\end{array}$ & $\begin{array}{l}\text { ACGH } \\
\text { E }\end{array}$ & $\begin{array}{l}22 \text { Jun, } 10 \text { Jul, } 13 \text { Aug, } 8 \text { Sep } \\
31 \text { Jul }\end{array}$ \\
\hline 7 & $\begin{array}{l}\text { Alto } 100 \text { SL } \\
\text { + Bravo Weather Stik } 6 \text { SC } \\
\text { Bravo Weather Stik } 6 \text { SC }\end{array}$ & $\begin{array}{l}5.5 \mathrm{fl} \mathrm{oz} \\
24 \mathrm{fl} \mathrm{oz} \\
24 \mathrm{fl} \mathrm{oz}\end{array}$ & $\begin{array}{l}\text { ACGH } \\
\text { E }\end{array}$ & $\begin{array}{l}22 \text { Jun, } 10 \text { Jul, } 13 \text { Aug, } 8 \text { Sep } \\
\text { 31 Jul }\end{array}$ \\
\hline 8 & $\begin{array}{l}\text { Miravis 1.67 SC } \\
\text { Bravo Weather Stik } 6 \text { SC }\end{array}$ & $\begin{array}{l}3.42 \mathrm{fl} \mathrm{oz} \\
24 \mathrm{fl} \mathrm{oz}\end{array}$ & $\begin{array}{l}\text { ACGH } \\
\text { E }\end{array}$ & $\begin{array}{l}22 \text { Jun, } 10 \text { Jul, } 13 \text { Aug, } 8 \text { Sep } \\
31 \text { Jul }\end{array}$ \\
\hline 9 & $\begin{array}{l}\text { Aprovia EC } \\
\text { Bravo Weather Stik } 6 \text { SC }\end{array}$ & $\begin{array}{l}10.5 \mathrm{fl} \mathrm{oz} \\
24 \mathrm{fl} \mathrm{oz}\end{array}$ & $\begin{array}{l}\text { ACGH } \\
\text { E }\end{array}$ & $\begin{array}{l}22 \text { Jun, } 10 \text { Jul, } 13 \text { Aug, } 8 \text { Sep } \\
31 \text { Jul }\end{array}$ \\
\hline 10 & $\begin{array}{l}\text { Elatus } 45 \mathrm{WG} \\
\text { Bravo Weather Stik } 6 \mathrm{SC}\end{array}$ & $\begin{array}{l}7.3 \mathrm{oz} \mathrm{wt} \\
24 \mathrm{fl} \mathrm{oz} \\
\end{array}$ & $\begin{array}{l}\text { ACGH } \\
\text { E }\end{array}$ & $\begin{array}{l}22 \text { Jun, } 10 \text { Jul, } 13 \text { Aug, } 8 \text { Sep } \\
31 \text { Jul }\end{array}$ \\
\hline 11 & $\begin{array}{l}\text { A19649 (H) SC } \\
\text { Bravo Weather Stik } 6 \text { SC }\end{array}$ & $\begin{array}{l}2.75 \mathrm{fl} \mathrm{oz} \\
24 \mathrm{fl} \mathrm{oz}\end{array}$ & $\begin{array}{l}\text { ACGH } \\
\text { E }\end{array}$ & $\begin{array}{l}\text { 22 Jun, } 10 \text { Jul, } 13 \text { Aug, } 8 \text { Sep } \\
\text { 31 Jul }\end{array}$ \\
\hline 12 & $\begin{array}{l}\text { Alto } 100 \text { SL } \\
\text { + Bravo Weather Stik } 6 \text { SC } \\
\text { Bravo Weather Stik } 6 \text { SC } \\
\text { A19649 (H) SC } \\
\text { + Elatus 45 WG } \\
\text { Bravo Weather Stik 6 SC }\end{array}$ & $\begin{array}{l}5.5 \mathrm{fl} \mathrm{oz} \\
16 \mathrm{fl} \mathrm{oz} \\
24 \mathrm{fl} \mathrm{oz} \\
3.42 \mathrm{fl} \mathrm{oz} \\
9.5 \mathrm{oz} \mathrm{wt} \\
24 \mathrm{fl} \mathrm{oz} \\
\end{array}$ & $\begin{array}{l}\text { A } \\
\text { B } \\
\text { DG } \\
\text { I }\end{array}$ & $\begin{array}{l}22 \text { Jun } \\
1 \text { Jul } \\
17 \text { Jul, } 13 \text { Aug } \\
14 \text { Sep }\end{array}$ \\
\hline 13 & $\begin{array}{l}\text { Elatus } 45 \mathrm{WG} \\
\text { Elatus } 45 \mathrm{WG} \\
\text { + A19649 (H) SC } \\
\text { Bravo Weather Stik 6 SC }\end{array}$ & $\begin{array}{l}7.3 \mathrm{oz} \mathrm{wt} \\
7.3 \mathrm{oz} \mathrm{wt} \\
3.42 \mathrm{fl} \mathrm{oz} \\
24 \mathrm{fl} \mathrm{oz}\end{array}$ & $\begin{array}{l}\mathrm{A} \\
\mathrm{CF} \\
\mathrm{HJ}\end{array}$ & $\begin{array}{l}22 \text { Jun } \\
10 \mathrm{Jul}, 6 \mathrm{Aug} \\
8 \mathrm{Sep}, 21 \mathrm{Sep}\end{array}$ \\
\hline
\end{tabular}

SOIL TYPE: Kenansville loamy fine sand

SOIL FERTILITY REPORT (Jan 2020):

\begin{tabular}{|l|l|l|l|l|l|l|l|l|l|}
\hline $\mathbf{p H}$ & $\mathbf{P}(\mathbf{l b} / \mathbf{A})$ & $\mathbf{K}(\mathbf{l b} / \mathbf{A})$ & $\mathbf{C a}(\mathbf{l b} / \mathbf{A})$ & $\mathbf{M g}(\mathbf{l b} / \mathbf{A})$ & $\mathbf{Z n}(\mathbf{p p m})$ & $\mathbf{M n}(\mathbf{p p m})$ & $\mathbf{C u}(\mathbf{p p m})$ & $\mathbf{F e}(\mathbf{p p m})$ & $\mathbf{B}(\mathbf{p p m})$ \\
\hline 6.4 & 72 & 86 & 574 & 68 & 0.6 & 3.3 & 0.2 & 16.0 & 0.1 \\
\hline
\end{tabular}

MAINTENANCE CHEMICAL PROGRAMS:

\begin{tabular}{|l|l|}
\hline Fertilizer & Standard \\
\hline Herbicides & Standard \\
\hline Insecticides & Admire Pro IF standard \\
\hline Fungicides & Proline IF \\
\hline Nematicides & None \\
\hline
\end{tabular}


MAINTENANCE CHEMICAL APPLICATIONS:

\begin{tabular}{|l|l|l|l|}
\hline Date & Type and target & Product and formulation & Rate/A \\
\hline $5 / 16 / 20$ & Insecticide & Admire Pro 4.6 SC & $8.5 \mathrm{fl} \mathrm{oz}$ \\
\hline $5 / 16 / 20$ & Fungicide & Proline 480 SC & $5.7 \mathrm{fl} \mathrm{oz}$ \\
\hline $5 / 17 / 20$ & Herbicide & Strongarm & $0.45 \mathrm{fl} \mathrm{oz}$ \\
\hline $5 / 17 / 20$ & Herbicide & Dual II MAGNUM & $1.5 \mathrm{pt}$ \\
\hline $5 / 17 / 20$ & Herbicide & Prowl H2O & $1.0 \mathrm{pt}$ \\
\hline $5 / 17 / 20$ & Fertility & Boron & $1.0 \mathrm{qt}$ \\
\hline $6 / 4 / 20$ & Insecticide & Orthene $75 \mathrm{~S}$ & $12 \mathrm{oz}$ \\
\hline $6 / 25 / 20$ & Herbicide & Basagran & $1.5 \mathrm{pt}$ \\
\hline $6 / 25 / 20$ & Herbicide & Fusilade DX & $1 \mathrm{pt}$ \\
\hline $7 / 7 / 20$ & Herbicide & Storm & $1.5 \mathrm{pt}$ \\
\hline $7 / 7 / 20$ & Herbicide & Basagran & $1 \mathrm{pt}$ \\
\hline $7 / 13 / 20$ & Fertility & Manganese & $2 \mathrm{qt}$ \\
\hline $7 / 13 / 20$ & Insecticide & Danitol 2.4 EC & $8 \mathrm{fl} \mathrm{oz}$ \\
\hline $7 / 16 / 20$ & Fertility & Landplaster & $1500 \mathrm{lb}$ \\
\hline $7 / 24 / 20$ & Insecticide & Danitol 2.4 EC & $8 \mathrm{fl} \mathrm{oz}$ \\
\hline $8 / 6 / 20$ & Herbicide & Tide 2 EC $(\mathrm{Clethodim})$ & $16 \mathrm{fl} \mathrm{oz}$ \\
\hline $8 / 18 / 20$ & Fungicide & Convoy & $29 \mathrm{fl} \mathrm{oz}$ \\
\hline & & & \\
\hline
\end{tabular}


Table 1. Effect of fungicide treatment on disease incidence in peanut (PTLFSPOT120, Suffolk, VA 2020).

\begin{tabular}{|c|c|c|c|c|c|}
\hline \multirow[b]{2}{*}{ Treatment, rate/A and application date ${ }^{\mathrm{z}}$} & \multicolumn{4}{|c|}{ Leaf spot $(1-10)^{y}$} & \multirow{2}{*}{$\begin{array}{l}\text { \% leaf } \\
\text { spot }^{w} \\
9 \text { Oct }\end{array}$} \\
\hline & 26 Aug & 13 Sep & 28 Sep & $\mathbf{A U D P C}^{\mathrm{x}}$ & \\
\hline 1. Untreated control & 2.0 & $3.8 \mathrm{a}$ & $6.5 \mathrm{a}$ & $128.6 \mathrm{a}$ & $93.8 \mathrm{a}$ \\
\hline $\begin{array}{ll}\text { 2. } & \text { Provysol SC } 5 \mathrm{fl} \mathrm{oz}(6 / 22,7 / 10,8 / 13,9 / 8) \\
& \text { Bravo Weather Stik } 6 \mathrm{SC} 24 \mathrm{fl} \mathrm{oz}(7 / 31) \\
\end{array}$ & 1.8 & $1.5 \mathrm{~cd}$ & $4.3 \mathrm{bc}$ & $72.4 \mathrm{~b}-\mathrm{d}$ & $57.5 \mathrm{c}-\mathrm{e}$ \\
\hline $\begin{array}{ll}\text { 3. } & \text { Provost Silver } 3.52 \text { SC } 12.8 \mathrm{fl} \mathrm{oz}(6 / 22,7 / 10,8 / 13,9 / 8) \\
& \text { Bravo Weather Stik } 6 \text { SC } 24 \mathrm{fl} \mathrm{oz}(7 / 31)\end{array}$ & 1.5 & $2.5 \mathrm{~b}$ & $4.0 \mathrm{bc}$ & $84.8 \mathrm{bc}$ & $45.0 \mathrm{e}$ \\
\hline $\begin{array}{ll}\text { 4. } & \text { Inspire EC } 7 \mathrm{fl} \mathrm{oz}(6 / 22,7 / 10,8 / 13,9 / 8) \\
& \text { Bravo Weather Stik } 6 \mathrm{SC} 24 \mathrm{fl} \mathrm{oz}(7 / 31) \\
\end{array}$ & 2.0 & $2.5 \mathrm{~b}$ & $4.5 \mathrm{~b}$ & $93.0 \mathrm{~b}$ & $77.5 \mathrm{a}-\mathrm{c}$ \\
\hline $\begin{array}{ll}\text { 5. } & \text { Alto } 100 \text { SL } 5.5 \mathrm{fl} \mathrm{oz}(6 / 22,7 / 10,8 / 13,9 / 8) \\
& \text { Bravo Weather Stik } 6 \text { SC } 24 \mathrm{fl} \mathrm{oz}(7 / 31)\end{array}$ & 1.8 & $2.3 \mathrm{bc}$ & $3.8 \mathrm{bc}$ & $81.0 \mathrm{bc}$ & $55.0 \mathrm{de}$ \\
\hline $\begin{array}{ll}\text { 6. } & \text { Bravo Top SC } 34 \mathrm{fl} \mathrm{oz}(6 / 22,7 / 10,8 / 13,9 / 8) \\
& \text { Bravo Weather Stik } 6 \text { SC } 24 \mathrm{fl} \mathrm{oz}(7 / 31) \\
\end{array}$ & 1.5 & $1.3 \mathrm{~d}$ & $4.8 \mathrm{~b}$ & $69.8 \mathrm{~cd}$ & $70.0 \mathrm{~b}-\mathrm{d}$ \\
\hline $\begin{array}{l}\text { 7. Alto } 100 \text { SL } 5.5 \mathrm{fl} \mathrm{oz} \\
\text { + Bravo Weather Stik } 6 \text { SC } 24 \mathrm{fl} \mathrm{oz}(6 / 22,7 / 10,8 / 13,9 / 8) \\
\text { Bravo Weather Stik } 6 \text { SC } 24 \mathrm{fl} \mathrm{oz}(7 / 31)\end{array}$ & 1.8 & $1.3 \mathrm{~d}$ & $2.5 \mathrm{de}$ & $55.1 \mathrm{de}$ & $40.0 \mathrm{e}$ \\
\hline $\begin{array}{ll}\text { 8. } & \text { Miravis } 1.67 \text { SC } 3.42 \mathrm{fl} \mathrm{oz}(6 / 22,7 / 10,8 / 13,9 / 8) \\
& \text { Bravo Weather Stik } 6 \text { SC } 24 \mathrm{fl} \mathrm{oz}(7 / 31) \\
\end{array}$ & 1.8 & $1.0 \mathrm{~d}$ & $1.5 \mathrm{ef}$ & $43.5 \mathrm{e}$ & $3.0 \mathrm{f}$ \\
\hline $\begin{array}{ll}9 . & \text { Aprovia EC } 10.5 \mathrm{fl} \mathrm{oz}(6 / 22,7 / 10,8 / 13,9 / 8) \\
& \text { Bravo Weather Stik } 6 \mathrm{SC} 24 \mathrm{fl} \mathrm{oz}(7 / 31) \\
\end{array}$ & 1.8 & $1.3 \mathrm{~d}$ & $4.3 \mathrm{bc}$ & $68.3 \mathrm{~cd}$ & $67.5 \mathrm{~b}-\mathrm{d}$ \\
\hline $\begin{array}{l}\text { 10. Elatus } 45 \text { WG } 7.3 \text { oz wt }(6 / 22,7 / 10,8 / 13,9 / 8) \\
\text { Bravo Weather Stik } 6 \text { SC } 24 \text { fl oz }(7 / 31)\end{array}$ & 1.5 & $1.8 \mathrm{~b}-\mathrm{d}$ & $4.5 \mathrm{~b}$ & $76.1 \mathrm{~b}-\mathrm{d}$ & $78.8 \mathrm{ab}$ \\
\hline $\begin{array}{l}\text { 11. A19649 (H) SC } 2.75 \mathrm{fl} \mathrm{oz}(6 / 22,7 / 10,8 / 13,9 / 8) \\
\text { Bravo Weather Stik } 6 \text { SC } 24 \mathrm{fl} \mathrm{oz}(7 / 31)\end{array}$ & 1.8 & $1.0 \mathrm{~d}$ & $1.0 \mathrm{f}$ & $39.8 \mathrm{e}$ & $3.3 \mathrm{f}$ \\
\hline $\begin{array}{l}\text { 12. Alto } 100 \text { SL } 5.5 \mathrm{fl} \mathrm{oz} \\
\text { + Bravo Weather Stik } 6 \mathrm{SC} 16 \mathrm{fl} \mathrm{oz}(6 / 22) \\
\text { Bravo Weather Stik } 6 \text { SC } 24 \mathrm{fl} \mathrm{oz}(7 / 1) \\
\text { A19649 (H) SC } 3.42 \mathrm{fl} \mathrm{oz} \\
\text { + Elatus 45 WG } 9.5 \mathrm{oz} \text { wt }(7 / 17,8 / 13) \\
\text { Bravo Weather Stik } 6 \text { SC } 24 \mathrm{fl} \mathrm{oz}(9 / 14)\end{array}$ & 1.5 & $1.0 \mathrm{~d}$ & $1.3 \mathrm{f}$ & $39.4 \mathrm{e}$ & $40.0 \mathrm{e}$ \\
\hline $\begin{array}{l}\text { 13. Elatus } 45 \mathrm{WG} 7.3 \mathrm{oz} \mathrm{wt}(6 / 22) \\
\text { Elatus } 45 \mathrm{WG} 7.3 \mathrm{oz} \mathrm{wt} \\
+\mathrm{A} 19649(\mathrm{H}) \mathrm{SC} 3.42 \mathrm{fl} \mathrm{oz}(7 / 10,8 / 6) \\
\text { Bravo Weather Stik } 6 \mathrm{SC} 24 \mathrm{fl} \mathrm{oz}(9 / 8,9 / 21)\end{array}$ & 1.8 & $1.0 \mathrm{~d}$ & $3.3 \mathrm{~cd}$ & $56.6 \mathrm{de}$ & $37.5 \mathrm{e}$ \\
\hline$P(\mathrm{~F})$ & 0.96 & $<0.0001$ & $<0.0001$ & $<0.0001$ & $<0.0001$ \\
\hline LSD & N.S. & 0.97 & 1.24 & 21.95 & 20.10 \\
\hline
\end{tabular}

z First fungicide for leaf spot program was applied at 35 DAP (22 Jun) and followed by applications as prescribed. All foliar treatments applied with Induce $0.125 \%$ v.v.

y Florida leaf spot scale: $1=$ no disease, $10=$ dead plants, Chiteka et al. (1988). Untreated control plots were observed for leaf spot on 7 Aug, little to no lesions observed.

$x$ AUDPC $=$ area under the disease progress curve.

${ }^{\mathrm{w}}$ Percent of leaves in two treatment rows with symptoms of leaf spot.

Means in a column followed by the same letter(s) are not significantly different according to Fisher's Protected LSD $(P=0.05)$. Arcsine transformation of percentage data was made in analysis to determine statistical significance. 
Table 2. Effect of treatment on soilborne disease and yield in peanut (PTLFSPOT120, Suffolk, VA 2020).

\begin{tabular}{|c|c|c|c|c|c|}
\hline \multirow[b]{2}{*}{ Treatment, rate/A and application date ${ }^{\mathrm{z}}$} & \multicolumn{4}{|c|}{ Sclerotinia blight ${ }^{y}$} & \multirow{2}{*}{$\begin{array}{c}\text { Yield } \\
(\mathbf{l b} / \mathbf{A})^{w}\end{array}$} \\
\hline & 13 Sep & 28 Sep & 15 Oct & AUDPC $^{\mathbf{x}}$ & \\
\hline 1. Untreated control & $8.5 \mathrm{c}-\mathrm{f}$ & $41.3 \mathrm{a}$ & $46.0 \mathrm{a}$ & $1114.8 \mathrm{a}$ & $1338 \mathrm{~cd}$ \\
\hline $\begin{array}{ll}\text { 2. } & \text { Provysol SC } 5 \mathrm{fl} \mathrm{oz}(6 / 22,7 / 10,8 / 13,9 / 8) \\
& \text { Bravo Weather Stik 6 SC } 24 \mathrm{fl} \mathrm{oz}(7 / 31)\end{array}$ & $16.8 \mathrm{a}$ & $37.5 \mathrm{ab}$ & $46.0 \mathrm{a}$ & $1116.6 \mathrm{a}$ & $729 \mathrm{~d}$ \\
\hline $\begin{array}{ll}\text { 3. } & \text { Provost Silver } 3.52 \text { SC } 12.8 \mathrm{fl} \mathrm{oz}(6 / 22,7 / 10,8 / 13,9 / 8) \\
& \text { Bravo Weather Stik } 6 \text { SC } 24 \mathrm{fl} \mathrm{oz}(7 / 31)\end{array}$ & $12.8 \mathrm{a}-\mathrm{d}$ & $38.3 \mathrm{a}$ & $45.5 \mathrm{a}$ & $1094.4 \mathrm{a}$ & $1559 \mathrm{c}$ \\
\hline $\begin{array}{ll}\text { 4. } & \text { Inspire EC } 7 \mathrm{fl} \mathrm{oz}(6 / 22,7 / 10,8 / 13,9 / 8) \\
& \text { Bravo Weather Stik } 6 \mathrm{SC} 24 \mathrm{fl} \mathrm{oz}(7 / 31) \\
\end{array}$ & $14.8 \mathrm{ab}$ & $39.8 \mathrm{a}$ & $46.0 \mathrm{a}$ & $1137.6 \mathrm{a}$ & $1060 \mathrm{~cd}$ \\
\hline $\begin{array}{ll}\text { 5. } & \text { Alto } 100 \text { SL } 5.5 \mathrm{fl} \mathrm{oz}(6 / 22,7 / 10,8 / 13,9 / 8) \\
& \text { Bravo Weather Stik } 6 \text { SC } 24 \mathrm{fl} \mathrm{oz}(7 / 31)\end{array}$ & $9.8 \mathrm{~b}-\mathrm{e}$ & $39.3 \mathrm{a}$ & $46.0 \mathrm{a}$ & $1092.1 \mathrm{a}$ & $1253 \mathrm{~cd}$ \\
\hline $\begin{array}{ll}\text { 6. } & \text { Bravo Top SC } 34 \mathrm{fl} \mathrm{oz}(6 / 22,7 / 10,8 / 13,9 / 8) \\
& \text { Bravo Weather Stik } 6 \text { SC } 24 \mathrm{fl} \mathrm{oz}(7 / 31)\end{array}$ & $14.0 \mathrm{a}-\mathrm{c}$ & $38.8 \mathrm{a}$ & $46.0 \mathrm{a}$ & $1116.0 \mathrm{a}$ & $910 \mathrm{~cd}$ \\
\hline $\begin{array}{l}\text { 7. Alto } 100 \text { SL } 5.5 \mathrm{fl} \mathrm{oz} \\
\text { + Bravo Weather Stik } 6 \text { SC } 24 \mathrm{fl} \mathrm{oz}(6 / 22,7 / 10,8 / 13,9 / 8) \\
\text { Bravo Weather Stik } 6 \text { SC } 24 \mathrm{fl} \mathrm{oz}(7 / 31)\end{array}$ & $14.3 \mathrm{a}-\mathrm{c}$ & $39.5 \mathrm{a}$ & $45.8 \mathrm{a}$ & $1127.8 \mathrm{a}$ & $908 \mathrm{~cd}$ \\
\hline $\begin{array}{ll}8 . & \text { Miravis } 1.67 \text { SC } 3.42 \mathrm{fl} \mathrm{oz}(6 / 22,7 / 10,8 / 13,9 / 8) \\
& \text { Bravo Weather Stik } 6 \text { SC } 24 \mathrm{fl} \mathrm{oz}(7 / 31)\end{array}$ & $3.0 \mathrm{fg}$ & $14.0 \mathrm{e}$ & $39.5 \mathrm{~cd}$ & $582.3 \mathrm{~d}$ & $2678 \mathrm{ab}$ \\
\hline $\begin{array}{ll}\text { 9. } & \text { Aprovia EC } 10.5 \mathrm{fl} \mathrm{oz}(6 / 22,7 / 10,8 / 13,9 / 8) \\
& \text { Bravo Weather Stik } 6 \text { SC } 24 \mathrm{fl} \mathrm{oz}(7 / 31)\end{array}$ & $7.0 \mathrm{~d}-\mathrm{g}$ & $28.8 \mathrm{bc}$ & $42.0 \mathrm{~b}$ & $869.5 \mathrm{~b}$ & $2278 \mathrm{~b}$ \\
\hline $\begin{array}{l}\text { 10. Elatus } 45 \text { WG } 7.3 \text { oz wt }(6 / 22,7 / 10,8 / 13,9 / 8) \\
\text { Bravo Weather Stik } 6 \text { SC } 24 \mathrm{fl} \mathrm{oz}(7 / 31)\end{array}$ & $5.3 \mathrm{e}-\mathrm{g}$ & $25.0 \mathrm{~cd}$ & $41.3 \mathrm{bc}$ & $790.0 \mathrm{bc}$ & $2607 \mathrm{ab}$ \\
\hline $\begin{array}{l}\text { 11. A19649 (H) SC } 2.75 \mathrm{fl} \mathrm{oz}(6 / 22,7 / 10,8 / 13,9 / 8) \\
\text { Bravo Weather Stik } 6 \text { SC } 24 \mathrm{fl} \mathrm{oz}(7 / 31)\end{array}$ & $3.3 \mathrm{fg}$ & $19.5 \mathrm{de}$ & $40.0 \mathrm{~b}-\mathrm{d}$ & $676.4 \mathrm{~cd}$ & $2361 \mathrm{~b}$ \\
\hline $\begin{array}{l}\text { 12. Alto } 100 \text { SL } 5.5 \mathrm{fl} \mathrm{oz} \\
\text { + Bravo Weather Stik } 6 \mathrm{SC} 16 \mathrm{fl} \mathrm{oz}(6 / 22) \\
\text { Bravo Weather Stik } 6 \mathrm{SC} 24 \mathrm{fl} \mathrm{oz}(7 / 1) \\
\text { A19649 (H) SC } 3.42 \mathrm{fl} \mathrm{oz} \\
\text { + Elatus } 45 \text { WG } 9.5 \mathrm{oz} \text { wt }(7 / 17,8 / 13) \\
\text { Bravo Weather Stik } 6 \mathrm{SC} 24 \mathrm{fl} \mathrm{oz}(9 / 14)\end{array}$ & $2.3 \mathrm{~g}$ & $15.8 \mathrm{e}$ & $39.0 \mathrm{~d}$ & $600.4 \mathrm{~d}$ & $3165 \mathrm{a}$ \\
\hline $\begin{array}{l}\text { 13. Elatus } 45 \mathrm{WG} 7.3 \mathrm{oz} \mathrm{wt}(6 / 22) \\
\text { Elatus } 45 \mathrm{WG} 7.3 \mathrm{oz} \mathrm{wt} \\
+ \text { A19649 (H) SC } 3.42 \mathrm{fl} \mathrm{oz}(7 / 10,8 / 6) \\
\text { Bravo Weather Stik } 6 \text { SC } 24 \mathrm{fl} \mathrm{oz}(9 / 8,9 / 21)\end{array}$ & $2.8 \mathrm{fg}$ & $19.3 \mathrm{de}$ & $40.3 \mathrm{~b}-\mathrm{d}$ & $670.8 \mathrm{~cd}$ & $2697 \mathrm{ab}$ \\
\hline$P(\mathrm{~F})$ & $<0.0001$ & $<0.0001$ & $<0.0001$ & $<0.0001$ & $<0.0001$ \\
\hline LSD & 5.78 & 8.80 & 2.22 & 169.34 & 683.0 \\
\hline
\end{tabular}

z First fungicide for leaf spot program was applied at 35 DAP (22 Jun) and followed by applications as prescribed. All foliar treatments applied with Induce $0.125 \%$ v.v.

y Counts of infection centers in the two center rows of each plot or a total of $70 \mathrm{ft}$ row. An infection center was a point with symptoms and/or signs of a disease and included 6 in. on either side of that point.

x AUDPC $=$ area under the disease progress curve.

${ }^{w}$ Yields are weight of peanuts with moisture content adjusted to 7\%. Peanuts were dug 19 Oct and harvested 4 Nov. Means in a column followed by the same letter(s) are not significantly different according to Fisher's Protected LSD $(P=0.05)$. 
TEST ID: PTLFSPOT220

PURPOSE: To compare fungicide treatments for control of peanut leaf spot and impact on yield

LOCATION: Tidewater Research Farm, Hare Rd., Suffolk, VA

CROP INFORMATION:

\begin{tabular}{|l|l|}
\hline Field & 29 \\
\hline Crop history & 2019 wheat/soy, 2018 wheat/soy, 2017 peanut \\
\hline Planting date & 16 May \\
\hline Variety & Sullivan \\
\hline Seeding rate & ca. 4 seed/row ft $(143 \mathrm{lb} / \mathrm{A})$ \\
\hline Plot length/width & $35^{\prime} \times 12^{\prime}$ \\
\hline Number of rows & 4 \\
\hline Row spacing & $36^{\prime \prime}$ \\
\hline Alleys (length between blocks) & $8^{\prime}$ \\
\hline Harvest date & 5 Nov \\
\hline
\end{tabular}

EXPERIMENTAL DESIGN: Randomized complete block with four replicates

\section{TREATMENT APPLICATION:}

\begin{tabular}{|l|l|}
\hline & Foliar spray \\
\hline Equipment & Tractor plot sprayer \\
\hline Pressure (psi) & $38 \mathrm{psi}$ \\
\hline Nozzle type & $8002 \mathrm{TwinJet}$ \\
\hline Volume (gal/A) & $19.88 \mathrm{gal} / \mathrm{A}$ \\
\hline Surfactant & NIS $0.125 \% \mathrm{v} / \mathrm{v}$ \\
\hline
\end{tabular}

\section{APPLICATION SCHEDULE:}

\begin{tabular}{|l|l|}
\hline A & 6" band at planting \\
\hline B & 45 DAP \\
\hline C & 60 DAP \\
\hline D & 75 DAP \\
\hline E & 90 DAP \\
\hline F & 105 DAP \\
\hline G & 120 DAP \\
\hline
\end{tabular}


TREATMENTS:

\begin{tabular}{|c|c|c|c|c|}
\hline Trt \# & Product and formulation & Rate/A & Appl. timing & Appl. date \\
\hline 1 & Untreated control & & & \\
\hline 2 & $\begin{array}{l}\text { Lucento 4.17 SC } \\
\text { Provost Silver 3.52 SC }\end{array}$ & $\begin{array}{l}5.5 \mathrm{fl} \mathrm{oz} \\
13 \mathrm{fl} \mathrm{oz}\end{array}$ & $\begin{array}{l}\text { CE } \\
\text { DF }\end{array}$ & $\begin{array}{l}15 \text { Jul. } 12 \text { Aug } \\
30 \text { Jul, } 28 \text { Aug }\end{array}$ \\
\hline 3 & $\begin{array}{l}\text { Miravis 1.67 SC } \\
\text { Provost Silver } 3.52 \mathrm{SC}\end{array}$ & $\begin{array}{l}3.4 \mathrm{fl} \mathrm{oz} \\
13 \mathrm{fl} \mathrm{oz}\end{array}$ & $\begin{array}{l}\mathrm{CE} \\
\mathrm{DF}\end{array}$ & $\begin{array}{l}15 \text { Jul, } 12 \text { Aug } \\
30 \text { Jul, } 28 \text { Aug }\end{array}$ \\
\hline 4 & $\begin{array}{l}\text { Provysol 3.34 SC } \\
\text { Provost Silver 3.52 SC }\end{array}$ & $\begin{array}{l}7 \mathrm{fl} \mathrm{oz} \\
13 \mathrm{fl} \mathrm{oz}\end{array}$ & $\begin{array}{l}\mathrm{CE} \\
\mathrm{DF}\end{array}$ & $\begin{array}{l}15 \mathrm{Jul}, 12 \text { Aug } \\
30 \mathrm{Jul}, 28 \text { Aug }\end{array}$ \\
\hline 5 & $\begin{array}{l}\text { Priaxor 4.17 SC } \\
\text { Provost Silver } 3.52 \mathrm{SC}\end{array}$ & $\begin{array}{l}4 \mathrm{fl} \mathrm{oz} \\
13 \mathrm{fl} \mathrm{oz}\end{array}$ & $\begin{array}{l}\mathrm{CE} \\
\mathrm{DF}\end{array}$ & $\begin{array}{l}15 \text { Jul, } 12 \text { Aug } \\
30 \text { Jul, } 28 \text { Aug }\end{array}$ \\
\hline 6 & $\begin{array}{l}\text { Provost Silver 3.52 SC } \\
\text { Lucento 4.17 SC }\end{array}$ & $\begin{array}{l}13 \mathrm{fl} \mathrm{oz} \\
5.5 \mathrm{fl} \mathrm{oz}\end{array}$ & $\begin{array}{l}\mathrm{CE} \\
\mathrm{DF}\end{array}$ & $\begin{array}{l}15 \text { Jul, } 12 \text { Aug } \\
30 \text { Jul, } 28 \text { Aug }\end{array}$ \\
\hline 7 & $\begin{array}{l}\text { Provost Silver } 3.52 \mathrm{SC} \\
\text { Miravis 1.67 SC }\end{array}$ & $\begin{array}{l}13 \mathrm{fl} \mathrm{oz} \\
3.4 \mathrm{fl} \mathrm{oz} \\
\end{array}$ & $\begin{array}{l}\mathrm{CE} \\
\mathrm{DF}\end{array}$ & $\begin{array}{l}15 \mathrm{Jul}, 12 \text { Aug } \\
30 \mathrm{Jul}, 28 \mathrm{Aug} \\
\end{array}$ \\
\hline 8 & $\begin{array}{l}\text { Lucento 4.17 SC } \\
\text { Provost Silver 3.52 SC } \\
\text { Bravo Weather Stik 6 SC }\end{array}$ & $\begin{array}{l}5.5 \mathrm{fl} \mathrm{oz} \\
13 \mathrm{fl} \mathrm{oz} \\
24 \mathrm{fl} \mathrm{oz}\end{array}$ & $\begin{array}{l}\text { CE } \\
\text { DF } \\
\text { G }\end{array}$ & $\begin{array}{l}15 \mathrm{Jul}, 12 \text { Aug } \\
30 \mathrm{Jul}, 28 \text { Aug } \\
5 \text { Oct }\end{array}$ \\
\hline 9 & $\begin{array}{l}\text { Lucento 4.17 SC } \\
\text { Provost Silver 3.52 SC } \\
\text { Topguard EQ 4.29 SC }\end{array}$ & $\begin{array}{l}5.5 \mathrm{fl} \mathrm{oz} \\
13 \mathrm{fl} \mathrm{oz} \\
5 \mathrm{fl} \mathrm{oz} \\
\end{array}$ & $\begin{array}{l}\text { CE } \\
\text { DF } \\
\text { G }\end{array}$ & $\begin{array}{l}15 \mathrm{Jul}, 12 \text { Aug } \\
30 \mathrm{Jul}, 28 \text { Aug } \\
5 \mathrm{Oct}\end{array}$ \\
\hline 10 & $\begin{array}{l}\text { Topguard EQ 4.29 SC } \\
\text { Lucento 4.17 SC } \\
\text { Provost Silver 3.52 SC }\end{array}$ & $\begin{array}{l}5 \mathrm{fl} \mathrm{oz} \\
5.5 \mathrm{fl} \mathrm{oz} \\
13 \mathrm{fl} \mathrm{oz} \\
\end{array}$ & $\begin{array}{l}\mathrm{B} \\
\mathrm{CE} \\
\mathrm{DF}\end{array}$ & $\begin{array}{l}30 \text { Jun } \\
15 \text { Jul, } 12 \text { Aug } \\
30 \text { Jul, } 28 \text { Aug }\end{array}$ \\
\hline 11 & $\begin{array}{l}\text { Topguard EQ 4.29 SC } \\
\text { Provost Silver 3.52 SC } \\
\text { Lucento 4.17 SC }\end{array}$ & $\begin{array}{l}5 \mathrm{fl} \mathrm{oz} \\
13 \mathrm{fl} \mathrm{oz} \\
5.5 \mathrm{fl} \mathrm{oz} \\
\end{array}$ & $\begin{array}{l}\mathrm{B} \\
\mathrm{CE} \\
\mathrm{DF}\end{array}$ & $\begin{array}{l}30 \text { Jun } \\
15 \text { Jul, } 12 \text { Aug } \\
30 \text { Jul, } 28 \text { Aug }\end{array}$ \\
\hline 12 & $\begin{array}{l}\text { Topguard 1.04 SC } \\
\text { Lucento 4.17 SC } \\
\text { Provost Silver 3.52 SC }\end{array}$ & $\begin{array}{l}14 \mathrm{fl} \mathrm{oz} \\
5.5 \mathrm{fl} \mathrm{oz} \\
13 \mathrm{fl} \mathrm{oz} \\
\end{array}$ & $\begin{array}{l}\mathrm{A} \\
\mathrm{CE} \\
\mathrm{DF}\end{array}$ & $\begin{array}{l}16 \text { May } \\
15 \text { Jul, } 12 \text { Aug } \\
30 \mathrm{Jul}, 28 \text { Aug } \\
\end{array}$ \\
\hline 13 & $\begin{array}{l}\text { Topguard EQ 4.29 SC } \\
\text { Lucento 4.17 SC } \\
\text { Provost Silver 3.52 SC }\end{array}$ & $\begin{array}{l}8 \mathrm{fl} \mathrm{oz} \\
5.5 \mathrm{fl} \mathrm{oz} \\
13 \mathrm{fl} \mathrm{oz} \\
\end{array}$ & $\begin{array}{l}\mathrm{A} \\
\mathrm{CE} \\
\mathrm{DF}\end{array}$ & $\begin{array}{l}16 \text { May } \\
15 \text { Jul, } 12 \text { Aug } \\
30 \mathrm{Jul}, 28 \text { Aug } \\
\end{array}$ \\
\hline 14 & Topguard 1.04 SC & $14 \mathrm{fl} \mathrm{oz}$ & $\mathrm{A}$ & 16 May \\
\hline 15 & Topguard EQ 4.29 SC & $8 \mathrm{fl} \mathrm{oz}$ & $\mathrm{A}$ & 16 May \\
\hline
\end{tabular}

SOIL TYPE: Goldsboro fine sandy loam

SOIL FERTILITY REPORT (Jan 2020):

\begin{tabular}{|l|l|l|l|l|l|l|l|l|l|}
\hline $\mathbf{p H}$ & $\mathbf{P}(\mathbf{l b} / \mathbf{A})$ & $\mathbf{K}(\mathbf{l b} / \mathbf{A})$ & $\mathbf{C a}(\mathbf{l b} / \mathbf{A})$ & $\mathbf{M g}(\mathbf{l b} / \mathbf{A})$ & $\mathbf{Z n}(\mathbf{p p m})$ & $\mathbf{M n}(\mathbf{p p m})$ & $\mathbf{C u}(\mathbf{p p m})$ & $\mathbf{F e}(\mathbf{p p m})$ & $\mathbf{B}(\mathbf{p p m})$ \\
\hline 5.9 & 31 & 79 & 345 & 43 & 0.4 & 2.5 & 0.3 & 14.3 & 0.1 \\
\hline
\end{tabular}

MAINTENANCE CHEMICAL PROGRAMS:

\begin{tabular}{|l|l|}
\hline Fertilizer & Standard \\
\hline Herbicides & Standard \\
\hline Insecticides & Admire Pro IF standard \\
\hline Fungicides & Proline IF \\
\hline Nematicides & None \\
\hline
\end{tabular}


MAINTENANCE CHEMICAL APPLICATIONS:

\begin{tabular}{|c|c|c|c|}
\hline Date & Type and target & Product and formulation & Rate/A \\
\hline $5 / 16 / 20$ & Insecticide & Admire Pro 4.6 SC & $8.5 \mathrm{fl} \mathrm{oz}$ \\
\hline $5 / 16 / 20$ & Fungicide & Proline $480 \mathrm{SC}$ & $5.7 \mathrm{fl} \mathrm{oz}$ \\
\hline $5 / 17 / 20$ & Herbicide & Strongarm & $0.45 \mathrm{fl} \mathrm{oz}$ \\
\hline $5 / 17 / 20$ & Herbicide & Dual II MAGNUM & $1.5 \mathrm{pt}$ \\
\hline $5 / 17 / 20$ & Herbicide & Prowl H2O & $1 \mathrm{pt}$ \\
\hline $5 / 17 / 20$ & Fertility & Boron & $1 \mathrm{qt}$ \\
\hline $6 / 4 / 20$ & Insecticide & Orthene $75 \mathrm{~S}$ & $12 \mathrm{oz}$ \\
\hline $6 / 8 / 20$ & Herbicide & Pursuit & $4 \mathrm{fl} \mathrm{oz}$ \\
\hline $6 / 25 / 20$ & Herbicide & Basagran & $1.5 \mathrm{pt}$ \\
\hline $6 / 25 / 20$ & Herbicide & Fusilade DX & $1 \mathrm{pt}$ \\
\hline $7 / 7 / 20$ & Herbicide & Storm & $1.5 \mathrm{pt}$ \\
\hline $7 / 7 / 20$ & Herbicide & Basagran & $1 \mathrm{pt}$ \\
\hline $7 / 13 / 20$ & Fertility & Manganese & $2 \mathrm{qt}$ \\
\hline $7 / 13 / 20$ & Insecticide & Danitol 2.4 EC & $8 \mathrm{fl} \mathrm{oz}$ \\
\hline $7 / 16 / 20$ & Fertility & Landplaster & $1500 \mathrm{lb}$ \\
\hline $7 / 24 / 20$ & Insecticide & Danitol 2.4 EC & $8 \mathrm{fl} \mathrm{oz}$ \\
\hline $8 / 6 / 20$ & Herbicide & Tide 2 EC (Clethodim) & $16 \mathrm{fl} \mathrm{oz}$ \\
\hline
\end{tabular}


Table 1. Effect of fungicide treatment on disease incidence in peanut (PTLFSPOT220, Suffolk, VA 2020).

\begin{tabular}{|c|c|c|c|c|c|}
\hline \multirow{2}{*}{$\begin{array}{l}\text { Treatment, rate/A } \\
\text { and application date } \\
\end{array}$} & \multicolumn{4}{|c|}{ Leaf spot $(1-10 \text { scale })^{y}$} & \multirow{2}{*}{$\begin{array}{l}\text { \% leaf } \\
\text { spot }^{w} \\
\text { 9 Oct }\end{array}$} \\
\hline & 24 Aug & 11 Sep & 30 Sep & AUDPC ${ }^{x}$ & \\
\hline 1. Untreated control & $4.0 \mathrm{a}$ & $6.5 \mathrm{a}$ & $9.0 \mathrm{a}$ & $241.8 \mathrm{a}$ & $100.0 \mathrm{a}$ \\
\hline $\begin{array}{ll}\text { 2. } & \text { Lucento 4.17 SC } 5.5 \mathrm{fl} \mathrm{oz}(7 / 15,8 / 12) \\
& \text { Provost Silver 3.52 SC } 13 \mathrm{fl} \mathrm{oz}(7 / 30,8 / 28) \\
\end{array}$ & $2.3 \mathrm{~cd}$ & $2.3 \mathrm{fg}$ & $5.3 \mathrm{~cd}$ & $111.8 \mathrm{fg}$ & $90.0 \mathrm{~b}$ \\
\hline $\begin{array}{ll}\text { 3. } & \text { Miravis } 1.67 \mathrm{SC} 3.4 \mathrm{fl} \mathrm{oz}(7 / 15,8 / 12) \\
& \text { Provost Silver } 3.52 \mathrm{SC} 13 \mathrm{fl} \mathrm{oz}(7 / 30,8 / 28) \\
\end{array}$ & $2.0 \mathrm{~d}$ & $2.0 \mathrm{~g}$ & $3.5 \mathrm{e}$ & $88.3 \mathrm{~g}$ & $57.5 \mathrm{~cd}$ \\
\hline $\begin{array}{ll}4 . & \text { Provysol 3.34 SC } 7 \mathrm{fl} \mathrm{oz}(7 / 15,8 / 12) \\
& \text { Provost Silver 3.52 SC } 13 \mathrm{fl} \mathrm{oz}(7 / 30,8 / 28) \\
\end{array}$ & $2.3 \mathrm{~cd}$ & $3.0 \mathrm{~d}-\mathrm{g}$ & $5.5 \mathrm{bc}$ & $128.0 \mathrm{~d}-\mathrm{f}$ & $85.0 \mathrm{~b}$ \\
\hline $\begin{array}{ll}5 . & \text { Priaxor 4.17 SC } 4 \mathrm{fl} \mathrm{oz}(7 / 15,8 / 12) \\
& \text { Provost Silver } 3.52 \mathrm{SC} 13 \mathrm{fl} \mathrm{oz}(7 / 30,8 / 28) \\
\end{array}$ & $2.3 \mathrm{~cd}$ & $3.5 \mathrm{de}$ & $4.3 \mathrm{de}$ & $125.4 \mathrm{~d}-\mathrm{f}$ & $75.0 \mathrm{bc}$ \\
\hline $\begin{array}{ll}\text { 6. } & \text { Provost Silver 3.52 SC } 13 \mathrm{fl} \mathrm{oz}(7 / 15,8 / 12) \\
& \text { Lucento 4.17 SC } 5.5 \mathrm{fl} \mathrm{oz}(7 / 30,8 / 28)\end{array}$ & $2.5 \mathrm{~b}-\mathrm{d}$ & $4.8 \mathrm{bc}$ & $6.5 \mathrm{~b}$ & $172.1 \mathrm{c}$ & $100.0 \mathrm{a}$ \\
\hline $\begin{array}{l}\text { 7. Provost Silver } 3.52 \mathrm{SC} 13 \mathrm{fl} \mathrm{oz}(7 / 15,8 / 12) \\
\text { Miravis } 1.67 \mathrm{SC} 3.4 \mathrm{fl} \mathrm{oz}(7 / 30,8 / 28)\end{array}$ & $2.0 \mathrm{~d}$ & $2.5 \mathrm{e}-\mathrm{g}$ & $2.3 \mathrm{f}$ & $85.6 \mathrm{~g}$ & $23.0 \mathrm{e}$ \\
\hline $\begin{array}{ll}\text { 8. } & \text { Lucento 4.17 SC } 5.5 \mathrm{fl} \mathrm{oz}(7 / 15,8 / 12) \\
& \text { Provost Silver 3.52 SC } 13 \mathrm{fl} \mathrm{oz}(7 / 30,8 / 28) \\
& \text { Bravo Weather Stik } 6 \text { SC } 24 \mathrm{fl} \mathrm{oz}(5 \mathrm{Oct})\end{array}$ & $2.8 \mathrm{bc}$ & $2.5 \mathrm{e}-\mathrm{g}$ & $5.0 \mathrm{~cd}$ & $118.5 \mathrm{ef}$ & $90.0 \mathrm{~b}$ \\
\hline $\begin{array}{ll}\text { 9. } & \text { Lucento 4.17 SC } 5.5 \mathrm{fl} \mathrm{oz}(7 / 15,8 / 12) \\
\text { Provost Silver 3.52 SC } 13 \mathrm{fl} \mathrm{oz}(7 / 30,8 / 28) \\
\text { Topguard EQ 4.29 SC } 5 \mathrm{fl} \mathrm{oz} \mathrm{(5} \mathrm{Oct)}\end{array}$ & $2.8 \mathrm{bc}$ & $3.5 \mathrm{de}$ & $4.8 \mathrm{~cd}$ & $134.6 \mathrm{~d}-\mathrm{f}$ & $85.0 \mathrm{~b}$ \\
\hline $\begin{array}{l}\text { 10. Topguard EQ 4.29 SC } 5 \mathrm{fl} \mathrm{oz}(6 / 30) \\
\text { Lucento 4.17 SC } 5.5 \mathrm{fl} \mathrm{oz}(7 / 15,8 / 12) \\
\text { Provost Silver 3.52 SC } 13 \mathrm{fl} \mathrm{oz}(7 / 30,8 / 28)\end{array}$ & $2.3 \mathrm{~cd}$ & $3.8 \mathrm{~cd}$ & $4.3 \mathrm{de}$ & $130.0 \mathrm{~d}-\mathrm{f}$ & $87.5 \mathrm{ab}$ \\
\hline $\begin{array}{l}\text { 11. Topguard EQ 4.29 SC } 5 \mathrm{fl} \mathrm{oz}(6 / 30) \\
\text { Provost Silver 3.52 SC } 13 \mathrm{fl} \mathrm{oz}(7 / 15,8 / 12) \\
\text { Lucento 4.17 SC } 5.5 \mathrm{fl} \mathrm{oz}(7 / 30,8 / 28)\end{array}$ & $2.0 \mathrm{~d}$ & $4.0 \mathrm{~cd}$ & $6.5 \mathrm{~b}$ & $153.8 \mathrm{~cd}$ & $100.0 \mathrm{a}$ \\
\hline $\begin{array}{l}\text { 12. Topguard 1.04 SC } 14 \mathrm{fl} \mathrm{oz}(5 / 16) \\
\text { Lucento 4.17 SC } 5.5 \mathrm{fl} \mathrm{oz}(7 / 15,8 / 12) \\
\text { Provost Silver } 3.52 \mathrm{SC} 13 \mathrm{fl} \mathrm{oz}(7 / 30,8 / 28)\end{array}$ & $2.0 \mathrm{~d}$ & $3.3 \mathrm{~d}-\mathrm{f}$ & $4.5 \mathrm{c}-\mathrm{e}$ & 120.9 ef & $45.0 \mathrm{de}$ \\
\hline $\begin{array}{l}\text { 13. Topguard EQ 4.29 SC } 8 \mathrm{fl} \mathrm{oz}(5 / 16) \\
\text { Lucento 4.17 SC } 5.5 \mathrm{fl} \mathrm{oz}(7 / 15,8 / 12) \\
\text { Provost Silver 3.52 SC } 13 \mathrm{fl} \mathrm{oz}(7 / 30,8 / 28)\end{array}$ & $2.3 \mathrm{~cd}$ & $4.0 \mathrm{~cd}$ & $5.3 \mathrm{~cd}$ & $144.1 \mathrm{c}-\mathrm{e}$ & $87.5 \mathrm{ab}$ \\
\hline 14. Topguard $1.04 \mathrm{SC} 14 \mathrm{fl} \mathrm{oz}(5 / 16)$ & $3.0 \mathrm{~b}$ & $5.3 \mathrm{~b}$ & $8.3 \mathrm{a}$ & $202.5 \mathrm{~b}$ & $100.0 \mathrm{a}$ \\
\hline 15. Topguard EQ 4.29 SC $8 \mathrm{fl} \mathrm{oz}(5 / 16)$ & $2.8 \mathrm{bc}$ & $5.5 \mathrm{ab}$ & $8.3 \mathrm{a}$ & $204.9 \mathrm{~b}$ & $100.0 \mathrm{a}$ \\
\hline$P(\mathrm{~F})$ & $<0.0001$ & $<0.0001$ & $<0.0001$ & $<0.0001$ & $<0.0001$ \\
\hline LSD & 0.58 & 1.22 & 1.02 & 29.88 & 8.61 \\
\hline
\end{tabular}

${ }^{\mathrm{z}}$ 6-inch band at planting treatments applied 16 May; first foliar treatment for leaf spot program was applied at 45 days after planting (30 Jun) and followed by applications on two-week timings as prescribed. All foliar applications were applied with Induce $0.125 \% \mathrm{v} / \mathrm{v}$.

y Florida leaf spot scale: $1=$ no disease, $10=$ dead plants, Chiteka et al. (1988). Untreated control plots were observed for leaf spot on 7 Aug, little to no lesions observed.

$x \quad$ AUDPC $=$ area under the disease progress curve.

${ }^{w}$ Percent of leaves in two treatment rows with symptoms of leaf spot.

Means in a column followed by the same letter(s) are not significantly different according to Fisher's Protected LSD $(P=0.05)$. Arcsine transformation of percentage data was made in analysis to determine statistical significance. 
Table 2. Effect of fungicide treatment on soilborne disease and yield in peanut (PTLFSPOT220, Suffolk, VA 2020).

\begin{tabular}{|c|c|c|c|}
\hline \multirow{2}{*}{$\begin{array}{l}\text { Treatment, rate/A } \\
\text { and application timing/date } \\
\text { zen }\end{array}$} & \multicolumn{2}{|c|}{ Sclerotinia blight ${ }^{\mathbf{y}}$} & \multirow{2}{*}{$\begin{array}{l}\text { Yield }^{x} \\
(\mathbf{l b} / \mathbf{A})\end{array}$} \\
\hline & 30 Sep & 16 Oct & \\
\hline 1. Untreated control & 0.5 & 11.5 & $1642 \mathrm{f}$ \\
\hline $\begin{array}{ll}\text { 2. } & \text { Lucento 4.17 SC } 5.5 \mathrm{fl} \mathrm{oz}(7 / 15,8 / 12) \\
& \text { Provost Silver } 3.52 \mathrm{SC} 13 \mathrm{fl} \mathrm{oz}(7 / 30,8 / 28) \\
\end{array}$ & 1.5 & 18.8 & $3999 \mathrm{~d}$ \\
\hline $\begin{array}{ll}\text { 3. } & \text { Miravis 1.67 SC } 3.4 \mathrm{fl} \mathrm{oz}(7 / 15,8 / 12) \\
& \text { Provost Silver } 3.52 \mathrm{SC} 13 \mathrm{fl} \mathrm{oz}(7 / 30,8 / 28) \\
\end{array}$ & 0.5 & 11.8 & $4996 \mathrm{ab}$ \\
\hline $\begin{array}{ll}4 . & \text { Provysol 3.34 SC } 7 \mathrm{fl} \mathrm{oz}(7 / 15,8 / 12) \\
& \text { Provost Silver } 3.52 \mathrm{SC} 13 \mathrm{fl} \mathrm{oz}(7 / 30,8 / 28) \\
\end{array}$ & 2.3 & 16.3 & $4081 \mathrm{~d}$ \\
\hline $\begin{array}{ll}\text { 5. Priaxor } 4.17 \mathrm{SC} 4 \mathrm{fl} \mathrm{oz}(7 / 15,8 / 12) \\
\text { Provost Silver } 3.52 \mathrm{SC} 13 \mathrm{fl} \mathrm{oz}(7 / 30,8 / 28)\end{array}$ & 0.8 & 9.3 & $4387 \mathrm{~b}-\mathrm{d}$ \\
\hline $\begin{array}{ll}\text { 6. Provost Silver } 3.52 \mathrm{SC} 13 \mathrm{fl} \mathrm{oz}(7 / 15,8 / 12) \\
\text { Lucento 4.17 SC } 5.5 \mathrm{fl} \mathrm{oz}(7 / 30,8 / 28)\end{array}$ & 0.8 & 13.5 & $4489 \mathrm{~b}-\mathrm{d}$ \\
\hline $\begin{array}{ll}\text { 7. Provost Silver } 3.52 \mathrm{SC} 13 \mathrm{fl} \mathrm{oz}(7 / 15,8 / 12) \\
\text { Miravis } 1.67 \mathrm{SC} 3.4 \mathrm{fl} \mathrm{oz}(7 / 30,8 / 28) \\
\end{array}$ & 0.3 & 10.5 & $5568 \mathrm{a}$ \\
\hline $\begin{array}{ll}\text { 8. } & \text { Lucento 4.17 SC } 5.5 \mathrm{fl} \mathrm{oz}(7 / 15,8 / 12) \\
& \text { Provost Silver 3.52 SC } 13 \mathrm{fl} \mathrm{oz}(7 / 30,8 / 28) \\
& \text { Bravo Weather Stik 6 SC } 24 \mathrm{fl} \mathrm{oz}(5 \mathrm{Oct}) \\
\end{array}$ & 1.3 & 12.0 & $4100 \mathrm{~cd}$ \\
\hline $\begin{array}{ll}\text { 9. Lucento 4.17 SC } 5.5 \mathrm{fl} \mathrm{oz}(7 / 15,8 / 12) \\
\text { Provost Silver 3.52 SC } 13 \mathrm{fl} \mathrm{oz}(7 / 30,8 / 28) \\
\text { Topguard EQ 4.29 SC } 5 \mathrm{fl} \mathrm{oz} \mathrm{(5} \mathrm{Oct)}\end{array}$ & 1.3 & 17.0 & $4704 \mathrm{~b}-\mathrm{d}$ \\
\hline $\begin{array}{l}\text { 10. Topguard EQ 4.29 SC } 5 \mathrm{fl} \mathrm{oz}(6 / 30) \\
\text { Lucento 4.17 SC } 5.5 \mathrm{fl} \mathrm{oz}(7 / 15,8 / 12) \\
\text { Provost Silver } 3.52 \mathrm{SC} 13 \mathrm{fl} \mathrm{oz}(7 / 30,8 / 28)\end{array}$ & 1.3 & 9.8 & $4600 \mathrm{~b}-\mathrm{d}$ \\
\hline $\begin{array}{l}\text { 11. Topguard EQ 4.29 SC } 5 \mathrm{fl} \mathrm{oz}(6 / 30) \\
\text { Provost Silver 3.52 SC } 13 \mathrm{fl} \mathrm{oz}(7 / 15,8 / 12) \\
\text { Lucento 4.17 SC } 5.5 \mathrm{fl} \mathrm{oz}(7 / 30,8 / 28)\end{array}$ & 2.3 & 14.8 & $4120 \mathrm{~cd}$ \\
\hline $\begin{array}{l}\text { 12. Topguard 1.04 SC } 14 \mathrm{fl} \mathrm{oz}(5 / 16) \\
\text { Lucento 4.17 SC } 5.5 \mathrm{fl} \mathrm{oz}(7 / 15,8 / 12) \\
\text { Provost Silver } 3.52 \mathrm{SC} 13 \mathrm{fl} \mathrm{oz}(7 / 30,8 / 28)\end{array}$ & 1.3 & 10.3 & $4724 b-d$ \\
\hline $\begin{array}{l}\text { 13. Topguard EQ 4.29 SC } 8 \mathrm{fl} \mathrm{oz}(5 / 16) \\
\text { Lucento 4.17 SC } 5.5 \mathrm{fl} \mathrm{oz}(7 / 15,8 / 12) \\
\text { Provost Silver } 3.52 \mathrm{SC} 13 \mathrm{fl} \mathrm{oz}(7 / 30,8 / 28)\end{array}$ & 1.3 & 12.3 & 4935 a-c \\
\hline 14. Topguard $1.04 \mathrm{SC} 14 \mathrm{fl} \mathrm{oz}(5 / 16)$ & 1.3 & 10.5 & $2270 \mathrm{ef}$ \\
\hline 15. Topguard EQ 4.29 SC $8 \mathrm{fl} \mathrm{oz}(5 / 16)$ & 1.0 & 11.0 & 2966 e \\
\hline$P(\mathrm{~F})$ & 0.51 & 0.57 & $<0.0001$ \\
\hline LSD & N.S. & N.S. & 837.0 \\
\hline
\end{tabular}

z 6-inch band at planting treatments applied 16 May; first foliar treatment for leaf spot program was applied at 45 days after planting (30 Jun) and followed by applications on two-week timings as prescribed. All foliar applications were applied with Induce $0.125 \% \mathrm{v} / \mathrm{v}$.

y Counts of infection centers in the two center rows of each plot or a total of $70 \mathrm{ft}$ row. An infection center was a point with symptoms and/or signs of a disease and included 6 in. on either side of that point. Plots were scouted for disease on $17 \mathrm{Jul}$ and 3 Aug but none observed.

$x$ Yields are weight of peanuts with moisture content adjusted to 7\%. Peanuts were dug 19 Oct and harvested 5 Nov. Means in a column followed by the same letter(s) are not significantly different according to Fisher's Protected LSD $(P=0.05)$. 
TEST ID: PTLFSPOT320

PURPOSE: To evaluate fungicide treatments for control of peanut leaf spot and impact on yield at three locations, site 1

LOCATION: Tidewater Research Farm, Hare Rd., Suffolk, VA

CROP INFORMATION:

\begin{tabular}{|l|l|}
\hline Field & 29 \\
\hline Crop history & 2019 wheat/soy, 2018 wheat/soy, 2017 peanut \\
\hline Planting date & 16 May \\
\hline Variety & Sullivan \\
\hline Seeding rate & ca. 4 seed/row ft $(143 \mathrm{lb} / \mathrm{A})$ \\
\hline Plot length/width & $35^{\prime} \times 12^{\prime}$ \\
\hline Number of rows & 4 \\
\hline Row spacing & $36^{\prime \prime}$ \\
\hline Alleys (length between blocks) & $8^{\prime}$ \\
\hline Harvest date & 5 Nov \\
\hline
\end{tabular}

EXPERIMENTAL DESIGN: Randomized complete block with four replicates

TREATMENT APPLICATION:

\begin{tabular}{|l|l|l|}
\hline & IF liquid & Foliar spray \\
\hline Equipment & $\mathrm{CO}_{2}$ sprayer & Tractor plot sprayer \\
\hline Pressure $(\mathbf{p s i})$ & & $38 \mathrm{psi}$ \\
\hline Nozzle type & $.075 \mathrm{microtube}$ & $8002 \mathrm{TwinJ} \mathrm{Jet}$ \\
\hline Volume $(\mathrm{gal} / \mathrm{A})$ & $5 \mathrm{gal} / \mathrm{A}$ & $19.88 \mathrm{gal} / \mathrm{A}$ \\
\hline Surfactant & & NIS $0.125 \% \mathrm{v} / \mathrm{v}$ \\
\hline
\end{tabular}

\section{APPLICATION SCHEDULE:}

\begin{tabular}{|l|l|}
\hline A & In-furrow (at planting) \\
\hline B & Pegging (49 DAP) \\
\hline C & R3 (63 DAP) \\
\hline D & R3 + 2 weeks (77 DAP) \\
\hline E & R3 + 4 weeks (91 DAP) \\
\hline F & R3 + 6 weeks (105 DAP) \\
\hline G & R3 + 8 weeks (119 DAP) \\
\hline
\end{tabular}

\section{TREATMENTS:}

\begin{tabular}{|l|l|l|l|l|}
\hline Trt \# & Product and formulation & Rate/A & Appl. timing & Appl. date \\
\hline 1 & Admire Pro 4.6 SC & $8.5 \mathrm{fl} \mathrm{oz}$ & & \\
& + Proline 480 SC & $5.7 \mathrm{fl} \mathrm{oz}$ & A & 16 May \\
\hline 2 & Admire Pro 4.6 SC & $8.5 \mathrm{fl} \mathrm{oz}$ & & \\
& + Proline 480 SC & $5.7 \mathrm{fl} \mathrm{oz}$ & A & 16 May \\
& Elatus 45 WG & $9.5 \mathrm{oz} \mathrm{wt}$ & & \\
& + Miravis 1.67 SC & $3.4 \mathrm{fl} \mathrm{oz}$ & CE & $17 \mathrm{Jul}, 13 \mathrm{Aug}$ \\
& Bravo Weather Stik & $24 \mathrm{fl} \mathrm{oz}$ & $\mathrm{G}$ & $14 \mathrm{Sep}$ \\
\hline 3 & Admire Pro 4.6 SC & $8.5 \mathrm{fl} \mathrm{oz}$ & & \\
& + Proline 480 SC & $5.7 \mathrm{fl} \mathrm{oz}$ & A & $16 \mathrm{May}$ \\
& Elatus 45 WG & $9.5 \mathrm{oz} \mathrm{wt}$ & & \\
& + Miravis 1.67 SC & $3.4 \mathrm{fl} \mathrm{oz}$ & DF & 31 Jul, 28 Aug \\
& Bravo Weather Stik & $24 \mathrm{fl} \mathrm{oz}$ & G & $14 \mathrm{Sep}$ \\
\hline
\end{tabular}




\begin{tabular}{|c|c|c|c|c|}
\hline 4 & $\begin{array}{l}\text { Admire Pro 4.6SC } \\
\text { + Proline } 480 \mathrm{SC} \\
\text { Miravis 1.67 SC } \\
\text { + Tebuconazole } 3.6 \mathrm{~F} \\
\text { Bravo Weather Stik }\end{array}$ & $\begin{array}{l}8.5 \mathrm{fl} \mathrm{oz} \\
5.7 \mathrm{fl} \mathrm{oz} \\
3.4 \mathrm{fl} \mathrm{oz} \\
7.2 \mathrm{fl} \mathrm{oz} \\
24 \mathrm{fl} \mathrm{oz} \\
\end{array}$ & $\begin{array}{l}\mathrm{A} \\
\mathrm{CE} \\
\mathrm{G}\end{array}$ & $\begin{array}{l}16 \text { May } \\
17 \text { Jul, } 13 \text { Aug } \\
14 \text { Sep }\end{array}$ \\
\hline 5 & $\begin{array}{l}\text { Admire Pro 4.6 SC } \\
\text { + Proline 480 SC } \\
\text { Miravis 1.67 SC } \\
\text { Elatus 45 WG } \\
\text { Bravo Weather Stik } \\
\end{array}$ & $\begin{array}{l}8.5 \mathrm{fl} \mathrm{oz} \\
5.7 \mathrm{fl} \mathrm{oz} \\
3.4 \mathrm{fl} \mathrm{oz} \\
9.5 \mathrm{oz} \mathrm{wt} \\
24 \mathrm{fl} \mathrm{oz} \\
\end{array}$ & $\begin{array}{l}\text { A } \\
\text { CE } \\
\text { DF } \\
G \\
\end{array}$ & $\begin{array}{l}16 \text { May } \\
17 \text { Jul, } 13 \text { Aug } \\
31 \text { Jul, } 28 \text { Aug } \\
14 \text { Sep }\end{array}$ \\
\hline 6 & $\begin{array}{l}\text { Admire Pro 4.6 SC } \\
\text { + Proline 480 SC } \\
\text { Domark 230 ME } \\
\text { + Bravo Weather Stik } \\
\text { Bravo Weather Stik } \\
\text { + Tebuconazole 3.6 F } \\
\text { Bravo Weather Stik }\end{array}$ & $\begin{array}{l}8.5 \mathrm{fl} \mathrm{oz} \\
5.7 \mathrm{fl} \mathrm{oz} \\
2.5 \mathrm{fl} \mathrm{oz} \\
16 \mathrm{fl} \mathrm{oz} \\
24 \mathrm{fl} \mathrm{oz} \\
7.2 \mathrm{fl} \mathrm{oz} \\
24 \mathrm{fl} \mathrm{oz}\end{array}$ & $\begin{array}{l}\mathrm{A} \\
\mathrm{CE} \\
\mathrm{DF} \\
\mathrm{G}\end{array}$ & $\begin{array}{l}16 \text { May } \\
17 \text { Jul, } 13 \text { Aug } \\
31 \text { Jul, } 28 \text { Aug } \\
14 \text { Sep }\end{array}$ \\
\hline 7 & $\begin{array}{l}\text { Admire Pro } 4.6 \mathrm{SC} \\
+ \text { Proline } 480 \mathrm{SC} \\
\text { Miravis } 1.67 \mathrm{SC} \\
\text { Omega } 500 \mathrm{~F} \\
\text { Bravo Weather Stik }\end{array}$ & $\begin{array}{l}8.5 \mathrm{fl} \mathrm{oz} \\
5.7 \mathrm{fl} \mathrm{oz} \\
3.4 \mathrm{fl} \mathrm{oz} \\
24 \mathrm{fl} \mathrm{oz} \\
24 \mathrm{fl} \mathrm{oz}\end{array}$ & $\begin{array}{l}\text { A } \\
\text { CE } \\
\text { DF } \\
G\end{array}$ & $\begin{array}{l}16 \text { May } \\
17 \text { Jul, } 13 \text { Aug } \\
31 \text { Jul, } 28 \text { Aug } \\
14 \text { Sep }\end{array}$ \\
\hline 8 & $\begin{array}{l}\text { Admire Pro 4.6 SC } \\
\text { + Proline 480 SC } \\
\text { Elatus 45 WG } \\
\text { Bravo Weather Stik } \\
+ \text { Tebuconazole 3.6 F } \\
\text { Bravo Weather Stik }\end{array}$ & $\begin{array}{l}8.5 \mathrm{fl} \mathrm{oz} \\
5.7 \mathrm{fl} \mathrm{oz} \\
9.5 \mathrm{oz} / \mathrm{wt} \\
24 \mathrm{fl} \mathrm{oz} \\
7.2 \mathrm{fl} \mathrm{oz} \\
24 \mathrm{fl} \mathrm{oz} \\
\end{array}$ & $\begin{array}{l}\text { A } \\
\text { CE } \\
\text { DF } \\
\text { G } \\
\end{array}$ & $\begin{array}{l}16 \text { May } \\
17 \text { Jul, } 13 \text { Aug } \\
31 \text { Jul, } 28 \text { Aug } \\
14 \text { Sep }\end{array}$ \\
\hline 9 & $\begin{array}{l}\text { Admire Pro 4.6 SC } \\
\text { + Proline 480 SC } \\
\text { Priaxor 4.17 SC } \\
\text { Bravo Weather Stik } \\
\text { + Tebuconazole } 3.6 \mathrm{~F} \\
\text { Bravo Weather Stik }\end{array}$ & $\begin{array}{l}8.5 \mathrm{fl} \mathrm{oz} \\
5.7 \mathrm{fl} \mathrm{oz} \\
8 \mathrm{fl} \mathrm{oz} \\
24 \mathrm{fl} \mathrm{oz} \\
7.2 \mathrm{fl} \mathrm{oz} \\
24 \mathrm{fl} \mathrm{oz} \\
\end{array}$ & $\begin{array}{l}\mathrm{A} \\
\mathrm{CE} \\
\mathrm{DF} \\
\mathrm{G} \\
\end{array}$ & $\begin{array}{l}16 \text { May } \\
17 \text { Jul, } 13 \text { Aug } \\
31 \text { Jul, } 28 \text { Aug }\end{array}$ \\
\hline 10 & $\begin{array}{l}\text { Admire Pro 4.6 SC } \\
\text { + Proline 480 SC } \\
\text { Provost Silver 3.52 SC } \\
\text { Bravo Weather Stik } \\
\text { + Tebuconazole } 3.6 \text { F } \\
\text { Bravo Weather Stik }\end{array}$ & $\begin{array}{l}8.5 \mathrm{fl} \mathrm{oz} \\
5.7 \mathrm{fl} \mathrm{oz} \\
13 \mathrm{fl} \mathrm{oz} \\
24 \mathrm{fl} \mathrm{oz} \\
7.2 \mathrm{fl} \mathrm{oz} \\
24 \mathrm{fl} \mathrm{oz}\end{array}$ & $\begin{array}{l}\mathrm{A} \\
\mathrm{CE} \\
\mathrm{DF} \\
\mathrm{G}\end{array}$ & $\begin{array}{l}16 \text { May } \\
17 \text { Jul, } 13 \text { Aug } \\
31 \text { Jul, } 28 \text { Aug } \\
14 \text { Sep }\end{array}$ \\
\hline 11 & $\begin{array}{l}\text { Admire Pro 4.6 SC } \\
\text { + Proline 480 SC } \\
\text { Lucento 4.17 SC } \\
\text { Bravo Weather Stik } \\
\text { + Tebuconazole } 3.6 \mathrm{~F} \\
\text { Bravo Weather Stik }\end{array}$ & $\begin{array}{l}8.5 \mathrm{fl} \mathrm{oz} \\
5.7 \mathrm{fl} \mathrm{oz} \\
5.5 \mathrm{fl} \mathrm{oz} \\
24 \mathrm{fl} \mathrm{oz} \\
7.2 \mathrm{fl} \mathrm{oz} \\
24 \mathrm{fl} \mathrm{oz} \\
\end{array}$ & $\begin{array}{l}\mathrm{A} \\
\mathrm{CE} \\
\mathrm{DF} \\
\mathrm{G}\end{array}$ & $\begin{array}{l}16 \text { May } \\
17 \text { Jul, } 13 \text { Aug } \\
31 \text { Jul, } 28 \text { Aug } \\
14 \text { Sep }\end{array}$ \\
\hline 12 & $\begin{array}{l}\text { Admire Pro 4.6 SC } \\
\text { + Proline 480 SC } \\
\text { Revytek SC } \\
\text { Bravo Weather Stik } \\
+ \text { Tebuconazole 3.6 F } \\
\text { Bravo Weather Stik }\end{array}$ & $\begin{array}{l}8.5 \mathrm{fl} \mathrm{oz} \\
5.7 \mathrm{fl} \mathrm{oz} \\
12 \mathrm{fl} \mathrm{oz} \\
24 \mathrm{fl} \mathrm{oz} \\
7.2 \mathrm{fl} \mathrm{oz} \\
24 \mathrm{fl} \mathrm{oz} \\
\end{array}$ & $\begin{array}{l}\text { A } \\
\text { CE } \\
\text { DF } \\
\text { G } \\
\end{array}$ & $\begin{array}{l}16 \text { May } \\
17 \text { Jul, } 13 \text { Aug } \\
31 \text { Jul, } 28 \text { Aug } \\
14 \text { Sep }\end{array}$ \\
\hline
\end{tabular}




\begin{tabular}{|c|c|c|c|c|}
\hline 13 & $\begin{array}{l}\text { Admire Pro 4.6 SC } \\
\text { + Proline 480 SC } \\
\text { Excalia SC } \\
\text { Bravo Weather Stik } \\
\text { + Tebuconazole 3.6 F } \\
\text { Bravo Weather Stik } \\
\end{array}$ & $\begin{array}{l}8.5 \mathrm{fl} \mathrm{oz} \\
5.7 \mathrm{fl} \mathrm{oz} \\
2.65 \mathrm{fl} \mathrm{oz} \\
24 \mathrm{fl} \mathrm{oz} \\
7.2 \mathrm{fl} \mathrm{oz} \\
24 \mathrm{fl} \mathrm{oz} \\
\end{array}$ & $\begin{array}{l}\text { A } \\
\text { CE } \\
\text { DF } \\
\mathrm{G} \\
\end{array}$ & $\begin{array}{l}16 \text { May } \\
17 \text { Jul, } 13 \text { Aug } \\
31 \text { Jul, } 28 \text { Aug } \\
14 \text { Sep }\end{array}$ \\
\hline 14 & $\begin{array}{l}\text { Admire Pro 4.6 SC } \\
\text { + Velum Prime 500 SC } \\
\text { Absolute 500 SC } \\
\text { Propulse 400 SC } \\
\text { + Elatus 45 WG } \\
\text { Bravo Weather Stik } \\
\text { Provost Silver 3.52 SC }\end{array}$ & $\begin{array}{l}8.5 \mathrm{fl} \mathrm{oz} \\
6.5 \mathrm{fl} \mathrm{oz} \\
3.5 \mathrm{fl} \mathrm{oz} \\
13.6 \mathrm{fl} \mathrm{oz} \\
7.3 \mathrm{wt} \mathrm{oz} \\
24 \mathrm{fl} \mathrm{oz} \\
13 \mathrm{fl} \mathrm{oz} \\
\end{array}$ & $\begin{array}{l}\text { A } \\
\text { C } \\
\text { D } \\
\text { EG } \\
\text { F }\end{array}$ & $\begin{array}{l}16 \text { May } \\
17 \text { Jul } \\
31 \text { Jul } \\
13 \text { Aug, } 14 \text { Sep } \\
28 \text { Aug }\end{array}$ \\
\hline 15 & $\begin{array}{l}\text { Admire Pro 4.6 SC } \\
\text { + Velum Prime 500 SC } \\
\text { Provost Silver 3.52 SC } \\
\text { Bravo Weather Stik } \\
\text { + Tebuconazole 3.6 F } \\
\text { Bravo Weather Stik } \\
\end{array}$ & $\begin{array}{l}8.5 \mathrm{fl} \mathrm{oz} \\
15.84 \mathrm{fl} \mathrm{oz} \\
13 \mathrm{fl} \mathrm{oz} \\
24 \mathrm{fl} \mathrm{oz} \\
7.2 \mathrm{fl} \mathrm{oz} \\
24 \mathrm{fl} \mathrm{oz} \\
\end{array}$ & $\begin{array}{l}\text { A } \\
\text { CE } \\
\text { DF } \\
\text { G } \\
\end{array}$ & $\begin{array}{l}16 \text { May } \\
17 \text { Jul, } 13 \text { Aug } \\
\text { 31 Jul, } 28 \text { Aug } \\
14 \text { Sep }\end{array}$ \\
\hline 16 & $\begin{array}{l}\text { Admire Pro 4.6 SC } \\
\text { + Velum Prime } 500 \mathrm{SC} \\
\text { Propulse 400 SC } \\
\text { Provost Silver } 3.52 \mathrm{SC} \\
\text { Bravo Weather Stik } \\
\text { + Tebuconazole 3.6 F } \\
\text { Bravo Weather Stik }\end{array}$ & $\begin{array}{l}8.5 \mathrm{fl} \mathrm{oz} \\
15.84 \mathrm{fl} \mathrm{oz} \\
13.6 \mathrm{fl} \mathrm{oz} \\
13 \mathrm{fl} \mathrm{oz} \\
24 \mathrm{fl} \mathrm{oz} \\
7.2 \mathrm{fl} \mathrm{oz} \\
24 \mathrm{fl} \mathrm{oz} \\
\end{array}$ & $\begin{array}{l}\text { A } \\
\text { B } \\
\text { CE } \\
\text { DF } \\
\text { G } \\
\end{array}$ & $\begin{array}{l}16 \text { May } \\
1 \text { Jul } \\
17 \text { Jul, } 13 \text { Aug } \\
31 \text { Jul, } 28 \text { Aug } \\
14 \text { Sep }\end{array}$ \\
\hline 17 & $\begin{array}{l}\text { Admire Pro 4.6 SC } \\
+ \text { Proline 480 SC } \\
\text { Propulse 400 SC } \\
\text { Provost Silver 3.52 SC } \\
\text { Bravo Weather Stik } \\
\text { + Tebuconazole 3.6 F } \\
\text { Bravo Weather Stik }\end{array}$ & $\begin{array}{l}8.5 \mathrm{fl} \mathrm{oz} \\
5.7 \mathrm{fl} \mathrm{oz} \\
13.6 \mathrm{fl} \mathrm{oz} \\
13 \mathrm{fl} \mathrm{oz} \\
24 \mathrm{fl} \mathrm{oz} \\
7.2 \mathrm{fl} \mathrm{oz} \\
24 \mathrm{fl} \mathrm{oz} \\
\end{array}$ & $\begin{array}{l}\text { A } \\
\text { B } \\
\text { CE } \\
\\
\text { DF } \\
\text { G } \\
\end{array}$ & $\begin{array}{l}16 \text { May } \\
1 \text { Jul } \\
17 \text { Jul, } 13 \text { Aug } \\
\text { 31 Jul, } 28 \text { Aug } \\
14 \text { Sep }\end{array}$ \\
\hline
\end{tabular}

SOIL TYPE: Goldsboro fine sandy loam

SOIL FERTILITY REPORT (Jan 2020):

\begin{tabular}{|l|l|l|l|l|l|l|l|l|l|}
\hline $\mathbf{p H}$ & $\mathbf{P}(\mathbf{l b} / \mathbf{A})$ & $\mathbf{K}(\mathbf{l b} / \mathbf{A})$ & $\mathbf{C a}(\mathbf{l b} / \mathbf{A})$ & $\mathbf{M g}(\mathbf{l b} / \mathbf{A})$ & $\mathbf{Z n}(\mathbf{p p m})$ & $\mathbf{M n}(\mathbf{p p m})$ & $\mathbf{C u}(\mathbf{p p m})$ & $\mathbf{F e}(\mathbf{p p m})$ & $\mathbf{B}(\mathbf{p p m})$ \\
\hline 5.9 & 31 & 79 & 345 & 43 & 0.4 & 2.5 & 0.3 & 14.3 & 0.1 \\
\hline
\end{tabular}

MAINTENANCE CHEMICAL PROGRAMS:

\begin{tabular}{|l|l|}
\hline Fertilizer & Standard \\
\hline Herbicides & Standard \\
\hline Insecticides & Standard \\
\hline Fungicides & None except treatments \\
\hline Nematicides & None except treatments \\
\hline
\end{tabular}


MAINTENANCE CHEMICAL APPLICATIONS:

\begin{tabular}{|l|l|l|l|}
\hline Date & Type and target & Product and formulation & Rate/A \\
\hline $5 / 17 / 20$ & Herbicide & Strongarm & $0.45 \mathrm{fl} \mathrm{oz}$ \\
\hline $5 / 17 / 20$ & Herbicide & Dual II MAGNUM & $1.5 \mathrm{pt}$ \\
\hline $5 / 17 / 20$ & Herbicide & Prowl H2O & $1.0 \mathrm{pt}$ \\
\hline $5 / 17 / 20$ & Fertility & Boron & $1.0 \mathrm{qt}$ \\
\hline $6 / 4 / 20$ & Insecticide & Orthene 75 S & $12 \mathrm{oz}$ \\
\hline $6 / 8 / 20$ & Herbicide & Pursuit & $4 \mathrm{fl} \mathrm{oz}$ \\
\hline $6 / 25 / 20$ & Herbicide & Basagran & $1.5 \mathrm{pt}$ \\
\hline $6 / 25 / 20$ & Herbicide & Fusilade DX & $1 \mathrm{pt}$ \\
\hline $7 / 7 / 20$ & Herbicide & Storm & $1.5 \mathrm{pt}$ \\
\hline $7 / 7 / 20$ & Herbicide & Basagran & $1 \mathrm{pt}$ \\
\hline $7 / 13 / 20$ & Fertility & Manganese & $2 \mathrm{qt}$ \\
\hline $7 / 13 / 20$ & Insecticide & Danitol 2.4 EC & $8 \mathrm{fl} \mathrm{oz}$ \\
\hline $7 / 16 / 20$ & Fertility & Landplaster & $1500 \mathrm{lb}$ \\
\hline $7 / 27 / 20$ & Insecticide & Danitol 2.4 EC & $8 \mathrm{fl} \mathrm{oz}$ \\
\hline $8 / 6 / 20$ & Herbicide & Tide 2 EC $(\mathrm{Clethodim})$ & $16 \mathrm{fl} \mathrm{oz}$ \\
\hline
\end{tabular}


Table 1. Effect of fungicide treatment on disease incidence in peanut (PTLFSPOT320, Suffolk, VA 2020).

\begin{tabular}{|c|c|c|c|c|c|}
\hline \multirow{2}{*}{$\begin{array}{l}\text { Treatment, rate/A } \\
\text { and application timing/date }{ }^{\mathrm{z}}\end{array}$} & \multicolumn{4}{|c|}{ Leaf spot $(1-10 \text { scale })^{y}$} & \multirow{2}{*}{$\begin{array}{l}\text { \% leaf } \\
\text { spot }^{w} \\
9 \text { Oct }\end{array}$} \\
\hline & 24 Aug & 11 Sep & 30 Sep & $\mathbf{A U D P C}^{\mathbf{x}}$ & \\
\hline $\begin{array}{l}\text { 1. Admire Pro 4.6 SC } 8.5 \mathrm{fl} \mathrm{oz} \\
\text { + Proline 480 SC } 5.7 \mathrm{fl} \mathrm{oz} \mathrm{(F)}\end{array}$ & $3.5 \mathrm{a}$ & $6.0 \mathrm{a}$ & $8.5 \mathrm{a}$ & $223.3 \mathrm{a}$ & $90.0 \mathrm{a}$ \\
\hline $\begin{array}{ll}\text { 2. } & \text { Admire Pro 4.6 SC } 8.5 \mathrm{fl} \mathrm{oz} \\
& + \text { Proline 480 SC } 5.7 \mathrm{fl} \mathrm{oz}(\mathrm{F}) \\
& \text { Elatus 45 WG } 9.5 \mathrm{oz} / \mathrm{wt} \\
& + \text { Miravis } 1.67 \mathrm{SC} 3.4 \mathrm{fl} \mathrm{oz}(7 / 17,8 / 13) \\
& \text { Bravo Weather Stik } 24 \mathrm{fl} \mathrm{oz}(9 / 14)\end{array}$ & $2.0 \mathrm{~b}-\mathrm{d}$ & $1.5 \mathrm{~h}$ & $5.0 \mathrm{bc}$ & $93.3 \mathrm{e}-\mathrm{g}$ & $46.3 \mathrm{~cd}$ \\
\hline $\begin{array}{ll}\text { 3. } & \text { Admire Pro 4.6 SC } 8.5 \mathrm{fl} \mathrm{oz} \\
\text { + Proline } 480 \mathrm{SC} 5.7 \mathrm{fl} \mathrm{oz}(\mathrm{F}) \\
\text { Elatus 45 WG } 9.5 \mathrm{oz} / \mathrm{wt} \\
\text { + Miravis } 1.67 \text { SC } 3.4 \mathrm{fl} \mathrm{oz}(7 / 31,8 / 28) \\
\text { Bravo Weather Stik } 24 \mathrm{fl} \mathrm{oz}(9 / 14)\end{array}$ & $2.8 \mathrm{ab}$ & $3.0 \mathrm{c}-\mathrm{f}$ & $1.8 \mathrm{~g}$ & $96.9 \mathrm{c}-\mathrm{g}$ & $21.3 \mathrm{~d}$ \\
\hline $\begin{array}{l}\text { 4. } \\
\text { Admire Pro 4.6 SC } 8.5 \mathrm{fl} \mathrm{oz} \\
\text { + Proline 480 SC } 5.7 \mathrm{fl} \mathrm{oz}(\mathrm{F}) \\
\text { Miravis } 1.67 \text { SC } 3.4 \mathrm{fl} \mathrm{oz} \\
\text { + Tebuconazole } 3.6 \mathrm{~F} 7.2 \mathrm{fl} \mathrm{oz}(7 / 31,8 / 28) \\
\text { Bravo Weather Stik } 24 \mathrm{fl} \mathrm{oz}(9 / 14)\end{array}$ & $1.8 \mathrm{~cd}$ & $1.5 \mathrm{~h}$ & $5.5 \mathrm{~b}$ & $95.8 \mathrm{~d}-\mathrm{g}$ & $77.5 \mathrm{ab}$ \\
\hline $\begin{array}{l}\text { 5. } \\
\text { Admire Pro 4.6 SC } 8.5 \mathrm{fl} \mathrm{oz} \\
\text { + Proline 480 SC } 5.7 \mathrm{fl} \mathrm{oz}(\mathrm{F}) \\
\text { Miravis 1.67 SC } 3.4 \mathrm{fl} \mathrm{oz}(7 / 17,8 / 13) \\
\text { Elatus 45 WG 9.5 oz/wt }(7 / 31,8 / 28) \\
\text { Bravo Weather Stik } 24 \mathrm{fl} \mathrm{oz}(9 / 14) \\
\end{array}$ & $1.8 \mathrm{~cd}$ & $1.8 \mathrm{gh}$ & $3.0 \mathrm{ef}$ & $76.6 \mathrm{~g}$ & $32.5 \mathrm{~d}$ \\
\hline $\begin{array}{ll}\text { 6. } & \text { Admire Pro 4.6 SC } 8.5 \mathrm{fl} \mathrm{oz} \\
& + \text { Proline } 480 \mathrm{SC} 5.7 \mathrm{fl} \mathrm{oz}(\mathrm{F}) \\
& \text { Domark } 230 \mathrm{ME} 2.5 \mathrm{fl} \mathrm{oz} \\
& + \text { Bravo Weather Stik } 16 \mathrm{fl} \mathrm{oz}(7 / 17,8 / 13) \\
& \text { Bravo Weather Stik } 24 \mathrm{fl} \mathrm{oz} \\
& + \text { Tebuconazole } 3.6 \mathrm{~F} 7.2 \mathrm{fl} \mathrm{oz}(7 / 31,8 / 28) \\
& \text { Bravo Weather Stik } 24 \mathrm{fl} \mathrm{oz}(9 / 14) \\
\end{array}$ & $2.0 \mathrm{~b}-\mathrm{d}$ & $3.3 \mathrm{c}-\mathrm{e}$ & $4.5 \mathrm{~b}-\mathrm{d}$ & $120.9 \mathrm{bc}$ & $55.0 \mathrm{~b}-\mathrm{d}$ \\
\hline $\begin{array}{l}\text { 7. } \\
\text { Admire Pro 4.6 SC } 8.5 \mathrm{fl} \mathrm{oz} \\
\text { + Proline 480 SC } 5.7 \mathrm{fl} \mathrm{oz}(\mathrm{F}) \\
\text { Miravis } 1.67 \mathrm{SC} 3.4 \mathrm{fl} \mathrm{oz}(7 / 17,8 / 13) \\
\text { Omega 500 F } 24 \mathrm{fl} \mathrm{oz}(7 / 31,8 / 28) \\
\text { Bravo Weather Stik } 24 \mathrm{fl} \mathrm{oz}(9 / 14) \\
\end{array}$ & $1.5 \mathrm{~d}$ & $1.8 \mathrm{gh}$ & $3.5 \mathrm{~d}-\mathrm{f}$ & $79.1 \mathrm{~g}$ & $52.5 \mathrm{~b}-\mathrm{d}$ \\
\hline $\begin{array}{ll}\text { 8. } & \text { Admire Pro 4.6 SC } 8.5 \mathrm{fl} \mathrm{oz} \\
& + \text { Proline } 480 \text { SC } 5.7 \mathrm{fl} \mathrm{oz}(\mathrm{F}) \\
& \text { Elatus } 45 \text { WG } 9.5 \mathrm{oz} / \mathrm{wt}(7 / 17,8 / 13) \\
& \text { Bravo Weather Stik } 24 \mathrm{fl} \mathrm{oz} \\
& + \text { Tebuconazole } 3.6 \mathrm{~F} 7.2 \mathrm{fl} \mathrm{oz}(7 / 31,8 / 28) \\
& \text { Bravo Weather Stik } 24 \mathrm{fl} \mathrm{oz}(9 / 14)\end{array}$ & $2.5 \mathrm{bc}$ & $3.5 \mathrm{~cd}$ & $3.5 \mathrm{~d}-\mathrm{f}$ & $120.5 \mathrm{~b}-\mathrm{d}$ & $47.5 \mathrm{~cd}$ \\
\hline $\begin{array}{l}\text { 9. } \\
\text { Admire Pro 4.6 SC } 8.5 \mathrm{fl} \mathrm{oz} \\
\text { + Proline 480 SC } 5.7 \mathrm{fl} \mathrm{oz}(\mathrm{F}) \\
\text { Priaxor 4.17 SC } 8 \mathrm{fl} \mathrm{oz}(7 / 17,8 / 13) \\
\text { Bravo Weather Stik } 24 \mathrm{fl} \mathrm{oz} \\
\text { + Tebuconazole } 3.6 \mathrm{~F} 7.2 \mathrm{fl} \mathrm{oz}(7 / 31,8 / 28) \\
\text { Bravo Weather Stik } 24 \mathrm{fl} \mathrm{oz}(9 / 14)\end{array}$ & $2.3 \mathrm{~b}-\mathrm{d}$ & $3.3 \mathrm{c}-\mathrm{e}$ & $3.5 \mathrm{~d}-\mathrm{f}$ & $113.6 \mathrm{c}-\mathrm{e}$ & $52.5 \mathrm{~b}-\mathrm{d}$ \\
\hline $\begin{array}{l}\text { 10. Admire Pro 4.6 SC } 8.5 \mathrm{fl} \mathrm{oz} \\
\text { + Proline 480 SC } 5.7 \mathrm{fl} \mathrm{oz}(\mathrm{F}) \\
\text { Provost Silver 3.52 SC } 13 \mathrm{fl} \mathrm{oz}(7 / 17,8 / 13) \\
\text { Bravo Weather Stik } 24 \mathrm{fl} \mathrm{oz} \\
\text { + Tebuconazole } 3.6 \mathrm{~F} 7.2 \mathrm{fl} \mathrm{oz}(7 / 31,8 / 28) \\
\text { Bravo Weather Stik } 24 \mathrm{fl} \mathrm{oz}(9 / 14)\end{array}$ & $1.5 \mathrm{~d}$ & $3.3 \mathrm{c}-\mathrm{e}$ & $3.3 \mathrm{ef}$ & $104.5 \mathrm{c}-\mathrm{f}$ & $57.5 \mathrm{~b}-\mathrm{d}$ \\
\hline $\begin{array}{l}\text { 11. Admire Pro 4.6 SC } 8.5 \mathrm{fl} \mathrm{oz} \\
\text { + Proline 480 SC } 5.7 \mathrm{fl} \mathrm{oz}(\mathrm{F}) \\
\text { Lucento 4.17 SC } 5.5 \mathrm{fl} \mathrm{oz}(7 / 13,8 / 13) \\
\text { Bravo Weather Stik } 24 \mathrm{fl} \mathrm{oz} \\
\text { + Tebuconazole 3.6 F } 7.2 \mathrm{fl} \mathrm{oz}(7 / 31,8 / 28) \\
\text { Bravo Weather Stik } 24 \mathrm{fl} \mathrm{oz}(9 / 14)\end{array}$ & $1.5 \mathrm{~d}$ & $3.8 \mathrm{bc}$ & $3.3 \mathrm{ef}$ & $113.8 \mathrm{c}-\mathrm{e}$ & $52.5 \mathrm{~b}-\mathrm{d}$ \\
\hline
\end{tabular}




\begin{tabular}{|c|c|c|c|c|c|}
\hline $\begin{array}{l}\text { 12. Admire Pro 4.6 SC } 8.5 \mathrm{fl} \mathrm{oz} \\
\text { + Proline 480 SC } 5.7 \mathrm{fl} \mathrm{oz} \mathrm{(F)} \\
\text { Revytek SC } 12 \mathrm{fl} \mathrm{oz}(7 / 17,8 / 13) \\
\text { Bravo Weather Stik } 24 \mathrm{fl} \mathrm{oz} \\
\text { + Tebuconazole } 3.6 \mathrm{~F} 7.2 \mathrm{fl} \mathrm{oz}(7 / 31,8 / 28) \\
\text { Bravo Weather Stik } 24 \mathrm{fl} \mathrm{oz}(9 / 14)\end{array}$ & $2.0 \mathrm{~b}-\mathrm{d}$ & $2.8 \mathrm{~d}-\mathrm{f}$ & $3.3 \mathrm{ef}$ & $99.8 \mathrm{c}-\mathrm{g}$ & $32.5 \mathrm{~d}$ \\
\hline $\begin{array}{l}\text { 13. Admire Pro 4.6 SC } 8.5 \mathrm{fl} \mathrm{oz} \\
+ \text { Proline 480 SC } 5.7 \mathrm{fl} \mathrm{oz} \mathrm{(F)} \\
\text { Excalia SC 2.65 fl oz }(7 / 17,8 / 13) \\
\text { Bravo Weather Stik } 24 \mathrm{fl} \mathrm{oz} \\
\text { + Tebuconazole 3.6 F } 7.2 \mathrm{fl} \mathrm{oz}(7 / 31,8 / 28) \\
\text { Bravo Weather Stik } 24 \mathrm{fl} \mathrm{oz}(9 / 14) \\
\end{array}$ & $2.3 \mathrm{~b}-\mathrm{d}$ & $4.5 \mathrm{~b}$ & $4.0 \mathrm{c}-\mathrm{e}$ & $141.5 \mathrm{~b}$ & $67.5 \mathrm{bc}$ \\
\hline $\begin{array}{l}\text { 14. Admire Pro 4.6 SC } 8.5 \mathrm{fl} \mathrm{oz} \\
\text { + Velum Prime } 500 \mathrm{SC} 15.84 \mathrm{fl} \mathrm{oz}(\mathrm{F}) \\
\text { Absolute 500 SC } 3.5 \mathrm{fl} \mathrm{oz}(7 / 17) \\
\text { Propulse 400 SC } 13.6 \mathrm{fl} \mathrm{oz} \\
\text { + Elatus 45 WG } 7.3 \mathrm{wt} / \mathrm{oz}(7 / 31) \\
\text { Bravo Weather Stik } 24 \mathrm{fl} \mathrm{oz}(8 / 13,9 / 14) \\
\text { Provost Silver } 3.52 \mathrm{SC} 13 \mathrm{fl} \mathrm{oz}(8 / 28) \\
\end{array}$ & $1.5 \mathrm{~d}$ & $2.3 \mathrm{f}-\mathrm{h}$ & $3.0 \mathrm{ef}$ & $83.6 \mathrm{fg}$ & $55.0 \mathrm{~b}-\mathrm{d}$ \\
\hline $\begin{array}{l}\text { 15. Admire Pro 4.6 SC } 8.5 \mathrm{fl} \mathrm{oz} \\
+ \text { Velum Prime 500 SC } 6.5 \mathrm{fl} \mathrm{oz}(\mathrm{F}) \\
\text { Provost Silver } 3.52 \mathrm{SC} 13 \mathrm{fl} \mathrm{oz}(7 / 17,8 / 13) \\
\text { Bravo Weather Stik } 24 \mathrm{fl} \mathrm{oz} \\
+ \text { Tebuconazole } 3.6 \mathrm{~F} 7.2 \mathrm{fl} \mathrm{oz}(7 / 31,8 / 28) \\
\text { Bravo Weather Stik } 24 \mathrm{fl} \mathrm{oz}(9 / 14)\end{array}$ & $1.5 \mathrm{~d}$ & $2.8 \mathrm{~d}-\mathrm{f}$ & $2.8 \mathrm{fg}$ & $90.5 \mathrm{e}-\mathrm{g}$ & $55.0 \mathrm{~b}-\mathrm{d}$ \\
\hline $\begin{array}{l}\text { 16. Admire Pro 4.6 SC } 8.5 \mathrm{fl} \mathrm{oz} \\
+ \text { Velum Prime 500 SC } 15.84 \mathrm{fl} \mathrm{oz}(\mathrm{F}) \\
\text { Propulse 400 SC } 13.6 \mathrm{fl} \mathrm{oz} \\
\text { Provost Silver 3.52 SC } 13 \mathrm{fl} \mathrm{oz}(7 / 13,8 / 13) \\
\text { Bravo Weather Stik } 24 \mathrm{fl} \mathrm{oz} \\
+ \text { Tebuconazole } 3.6 \mathrm{~F} 7.2 \mathrm{fl} \mathrm{oz}(7 / 31,8 / 28) \\
\text { Bravo Weather Stik } 24 \mathrm{fl} \mathrm{oz}(9 / 14)\end{array}$ & $2.0 \mathrm{~b}-\mathrm{d}$ & $2.3 \mathrm{f}-\mathrm{h}$ & $2.8 \mathrm{fg}$ & $85.8 \mathrm{fg}$ & $42.5 \mathrm{~cd}$ \\
\hline $\begin{array}{l}\text { 17. Admire Pro 4.6 SC } 8.5 \mathrm{fl} \mathrm{oz} \\
\text { + Proline 480 SC } 5.7 \mathrm{fl} \mathrm{oz} \mathrm{(F)} \\
\text { Propulse 400 SC } 13.6 \mathrm{fl} \mathrm{oz} \\
\text { Provost Silver } 3.52 \text { SC } 13 \mathrm{fl} \mathrm{oz} 7 / 17,8 / 13) \\
\text { Bravo Weather Stik } 24 \mathrm{fl} \mathrm{oz} \\
\text { + Tebuconazole } 3.6 \mathrm{~F} 7.2 \mathrm{fl} \mathrm{oz}(7 / 31,8 / 28) \\
\text { Bravo Weather Stik } 24 \mathrm{fl} \mathrm{oz}(9 / 14) \\
\end{array}$ & $1.8 \mathrm{~cd}$ & $2.5 \mathrm{e}-\mathrm{g}$ & $3.0 \mathrm{ef}$ & $90.5 \mathrm{e}-\mathrm{g}$ & $42.5 \mathrm{~cd}$ \\
\hline$P(\mathrm{~F})$ & 0.0003 & $<0.0001$ & $<0.0001$ & $<0.0001$ & 0.002 \\
\hline LSD & 0.80 & 0.98 & 1.11 & 25.06 & 31.12 \\
\hline
\end{tabular}

z (F) In-furrow (16 May); (P) broadcast at pegging (1 Jul). First fungicide for leaf spot program was applied at R3 (17 Jul) and followed by applications on two-week timings as prescribed. All foliar treatments applied with Induce $0.125 \% \mathrm{v} / \mathrm{v}$.

y Florida leaf spot scale: $1=$ no disease, $10=$ dead plants, Chiteka et al. (1988). Untreated control (Trt. 1) plots were observed for leaf spot on $7 \mathrm{Aug}$; little to no lesions observed.

$x$ AUDPC $=$ area under the disease progress curve.

${ }^{w}$ Percent of leaves in two treatment rows with symptoms of leaf spot. Means in a column followed by the same letter(s) are not significantly different according to Fisher's Protected LSD $(P=0.05)$. Arcsine transformation of percentage data was made in analysis to determine statistical significance. 
Table 2. Effect of fungicide treatment on soilborne disease and yield in peanut (PTLFSPOT320, Suffolk, VA 2020).

\begin{tabular}{|c|c|c|c|}
\hline \multirow{2}{*}{$\begin{array}{l}\text { Treatment, rate/A } \\
\text { and application timing/date }\end{array}$} & \multicolumn{2}{|c|}{ Sclerotinia blight $^{\mathbf{y}}$} & \multirow{2}{*}{$\begin{array}{c}\text { Yield } \\
(\mathbf{l b} / \mathbf{A})^{\mathrm{x}}\end{array}$} \\
\hline & 30 Sep & 16 Oct & \\
\hline $\begin{array}{l}\text { 1. Admire Pro 4.6 SC } 8.5 \mathrm{fl} \mathrm{oz} \\
+ \text { Proline 480 SC } 5.7 \mathrm{fl} \mathrm{oz}(\mathrm{F})\end{array}$ & $0.3 \mathrm{c}$ & $8.8 \mathrm{c}-\mathrm{e}$ & $1863 \mathrm{~g}$ \\
\hline $\begin{array}{ll}\text { 2. } & \text { Admire Pro 4.6 SC } 8.5 \mathrm{fl} \mathrm{oz} \\
& + \text { Proline } 480 \mathrm{SC} 5.7 \mathrm{fl} \mathrm{oz}(\mathrm{F}) \\
& \text { Elatus 45 WG } 9.5 \mathrm{oz} / \mathrm{wt} \\
& + \text { Miravis } 1.67 \mathrm{SC} 3.4 \mathrm{fl} \mathrm{oz}(7 / 17,8 / 13) \\
& \text { Bravo Weather Stik } 24 \mathrm{fl} \mathrm{oz}(9 / 14) \\
\end{array}$ & $0.8 \mathrm{bc}$ & $8.3 \mathrm{de}$ & $4949 a-c$ \\
\hline $\begin{array}{ll}\text { 3. } & \text { Admire Pro 4.6 SC } 8.5 \mathrm{fl} \mathrm{oz} \\
\text { + Proline } 480 \mathrm{SC} 5.7 \mathrm{fl} \mathrm{oz}(\mathrm{F}) \\
\text { Elatus 45 WG } 9.5 \mathrm{oz} / \mathrm{wt} \\
\text { + Miravis } 1.67 \text { SC } 3.4 \mathrm{fl} \mathrm{oz}(7 / 31,8 / 28) \\
\text { Bravo Weather Stik } 24 \mathrm{fl} \mathrm{oz}(9 / 14) \\
\end{array}$ & $0.3 \mathrm{c}$ & $4.0 \mathrm{e}$ & $5052 \mathrm{ab}$ \\
\hline $\begin{array}{l}\text { 4. } \\
\text { Admire Pro 4.6 SC } 8.5 \mathrm{fl} \mathrm{oz} \\
\text { + Proline 480 SC } 5.7 \mathrm{fl} \mathrm{oz}(\mathrm{F}) \\
\text { Miravis } 1.67 \text { SC } 3.4 \mathrm{fl} \mathrm{oz} \\
\text { + Tebuconazole } 3.6 \mathrm{~F} 7.2 \mathrm{fl} \mathrm{oz}(7 / 31,8 / 28) \\
\text { Bravo Weather Stik } 24 \mathrm{fl} \mathrm{oz}(9 / 14) \\
\end{array}$ & $0.3 \mathrm{c}$ & $7.5 \mathrm{de}$ & $4255 \mathrm{c}-\mathrm{f}$ \\
\hline $\begin{array}{ll}\text { 5. } & \text { Admire Pro 4.6 SC } 8.5 \mathrm{fl} \mathrm{oz} \\
\text { + Proline 480 SC } 5.7 \mathrm{fl} \mathrm{oz}(\mathrm{F}) \\
\text { Miravis 1.67 SC } 3.4 \mathrm{fl} \mathrm{oz}(7 / 17,8 / 13) \\
\text { Elatus 45 WG 9.5 oz/wt }(7 / 31,8 / 28) \\
\text { Bravo Weather Stik } 24 \mathrm{fl} \mathrm{oz}(9 / 14) \\
\end{array}$ & $0.3 \mathrm{c}$ & $3.8 \mathrm{e}$ & $5160 \mathrm{a}$ \\
\hline $\begin{array}{l}\text { 6. Admire Pro 4.6 SC } 8.5 \mathrm{fl} \mathrm{oz} \\
\text { + Proline } 480 \mathrm{SC} 5.7 \mathrm{fl} \mathrm{oz}(\mathrm{F}) \\
\text { Domark 230 ME } 2.5 \mathrm{fl} \mathrm{oz} \\
\text { + Bravo Weather Stik } 16 \mathrm{fl} \mathrm{oz}(7 / 17,8 / 13) \\
\text { Bravo Weather Stik } 24 \mathrm{fl} \mathrm{oz} \\
\text { + Tebuconazole } 3.6 \mathrm{~F} 7.2 \mathrm{fl} \mathrm{oz}(7 / 31,8 / 28) \\
\text { Bravo Weather Stik } 24 \mathrm{fl} \mathrm{oz}(9 / 14)\end{array}$ & $1.3 \mathrm{bc}$ & $8.5 \mathrm{de}$ & $4276 \mathrm{c}-\mathrm{f}$ \\
\hline $\begin{array}{ll}\text { 7. } & \text { Admire Pro 4.6 SC } 8.5 \mathrm{fl} \mathrm{oz} \\
\text { + Proline 480 SC } 5.7 \mathrm{fl} \mathrm{oz}(\mathrm{F}) \\
\text { Miravis } 1.67 \mathrm{SC} 3.4 \mathrm{fl} \mathrm{oz}(7 / 17,8 / 13) \\
\text { Omega 500 F } 24 \mathrm{fl} \mathrm{oz}(7 / 31,8 / 28) \\
\text { Bravo Weather Stik } 24 \mathrm{fl} \mathrm{oz}(9 / 14) \\
\end{array}$ & $1.0 \mathrm{bc}$ & $7.0 \mathrm{de}$ & $4720 \mathrm{a}-\mathrm{d}$ \\
\hline $\begin{array}{l}\text { 8. } \\
\text { Admire Pro 4.6 SC } 8.5 \mathrm{fl} \mathrm{oz} \\
\text { + Proline 480 SC } 5.7 \mathrm{fl} \mathrm{oz}(\mathrm{F}) \\
\text { Elatus 45 WG 9.5 oz/wt }(7 / 17,8 / 13) \\
\text { Bravo Weather Stik } 24 \mathrm{fl} \mathrm{oz} \\
\text { + Tebuconazole } 3.6 \mathrm{~F} 7.2 \mathrm{fl} \mathrm{oz}(7 / 31,8 / 28) \\
\text { Bravo Weather Stik } 24 \mathrm{fl} \mathrm{oz}(9 / 14)\end{array}$ & $2.3 \mathrm{a}-\mathrm{c}$ & $13.8 \mathrm{a}-\mathrm{d}$ & $3696 \mathrm{f}$ \\
\hline $\begin{array}{l}\text { 9. } \\
\text { Admire Pro 4.6 SC } 8.5 \mathrm{fl} \mathrm{oz} \\
\text { + Proline 480 SC } 5.7 \mathrm{fl} \mathrm{oz}(\mathrm{F}) \\
\text { Priaxor 4.17 SC } 8 \mathrm{fl} \mathrm{oz}(7 / 17,8 / 13) \\
\text { Bravo Weather Stik } 24 \mathrm{fl} \mathrm{oz} \\
\text { + Tebuconazole } 3.6 \mathrm{~F} 7.2 \mathrm{fl} \mathrm{oz}(7 / 31,8 / 28) \\
\text { Bravo Weather Stik } 24 \mathrm{fl} \mathrm{oz}(9 / 14)\end{array}$ & $1.0 \mathrm{bc}$ & $11.0 \mathrm{~b}-\mathrm{e}$ & 4507 a-e \\
\hline $\begin{array}{l}\text { 10. } \text { Admire Pro 4.6 SC } 8.5 \mathrm{fl} \mathrm{oz} \\
\text { + Proline 480 SC 5.7 fl oz (F) } \\
\text { Provost Silver 3.52 SC } 13 \mathrm{fl} \mathrm{oz}(7 / 17,8 / 13) \\
\text { Bravo Weather Stik } 24 \mathrm{fl} \mathrm{oz} \\
\text { + Tebuconazole } 3.6 \mathrm{~F} 7.2 \mathrm{fl} \mathrm{oz}(7 / 31,8 / 28) \\
\text { Bravo Weather Stik } 24 \mathrm{fl} \mathrm{oz}(9 / 14) \\
\end{array}$ & $2.8 \mathrm{ab}$ & $19.5 \mathrm{ab}$ & $4068 \mathrm{~d}-\mathrm{f}$ \\
\hline $\begin{array}{l}\text { 11. Admire Pro 4.6 SC } 8.5 \mathrm{fl} \mathrm{oz} \\
\text { + Proline 480 SC } 5.7 \mathrm{fl} \mathrm{oz} \mathrm{(F)} \\
\text { Lucento 4.17 SC } 5.5 \mathrm{fl} \mathrm{oz}(7 / 13,8 / 13) \\
\text { Bravo Weather Stik } 24 \mathrm{fl} \mathrm{oz} \\
\text { + Tebuconazole } 3.6 \mathrm{~F} 7.2 \mathrm{fl} \mathrm{oz}(7 / 31,8 / 28) \\
\text { Bravo Weather Stik } 24 \mathrm{fl} \mathrm{oz}(9 / 14) \\
\end{array}$ & $2.3 \mathrm{a}-\mathrm{c}$ & $19.0 \mathrm{ab}$ & $4222 \mathrm{~d}-\mathrm{f}$ \\
\hline
\end{tabular}




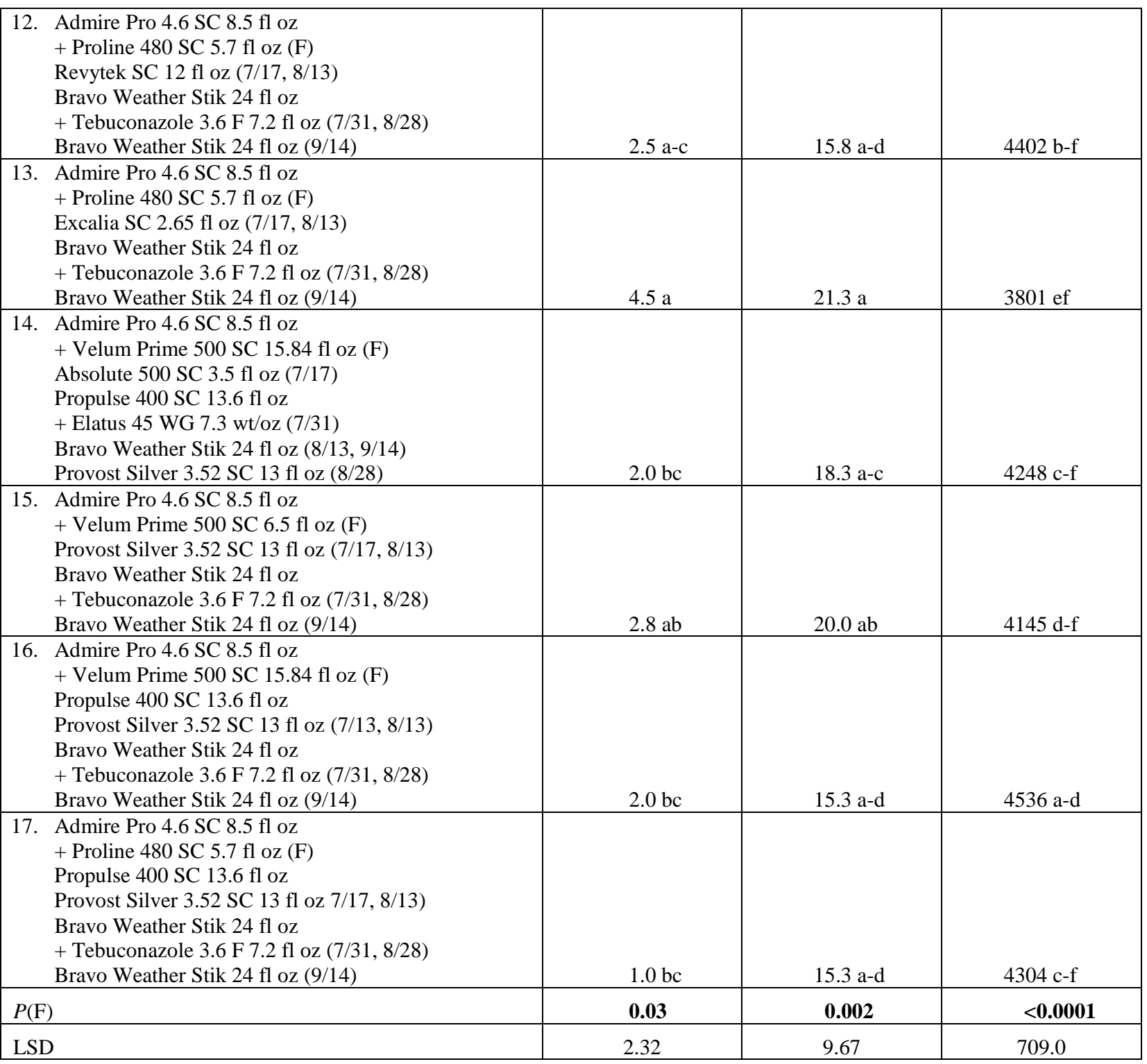

z (F) In-furrow (16 May); (P) broadcast at pegging (1 Jul). First fungicide for leaf spot program was applied at R3 (17 Jul) and followed by applications on two-week timings as prescribed. All foliar treatments applied with Induce $0.125 \% \mathrm{v} / \mathrm{v}$.

y Counts of infection centers in the two center rows of each plot or a total of $70 \mathrm{ft}$ row. An infection center was a point with symptoms and/or signs of a disease and included 6 in. on either side of that point.

$x$ Yields are weight of peanuts with moisture content adjusted to 7\%. Peanuts were dug 10 Oct and harvested 5 Nov. Means in a column followed by the same letter(s) are not significantly different according to Fisher's Protected LSD $(P=0.05)$. 
TEST ID: PTLFSPOT420

PURPOSE: To evaluate fungicide treatments for control of peanut leaf spot and Sclerotinia blight, and impact on yield at three locations, site 2

LOCATION: Tidewater Research Farm, Hare Rd., Suffolk, VA

CROP INFORMATION:

\begin{tabular}{|l|l|}
\hline Field & 34B \\
\hline Crop history & 2019 corn, 2018 cotton, 2017 peanut \\
\hline Planting date & 16 May \\
\hline Variety & Sullivan \\
\hline Seeding rate & ca. 4 seed/row ft $(143 \mathrm{lb} / \mathrm{A})$ \\
\hline Plot length/width & $35^{\prime} \times 12^{\prime}$ \\
\hline Number of rows & 4 \\
\hline Row spacing & $36^{\prime \prime}$ \\
\hline Alleys (length between blocks) & $8^{\prime}$ \\
\hline Harvest date & 4 Nov \\
\hline
\end{tabular}

EXPERIMENTAL DESIGN: Randomized complete block with four replicates

TREATMENT APPLICATION:

\begin{tabular}{|l|l|l|}
\hline & IF liquid & Foliar spray \\
\hline Equipment & $\mathrm{CO}_{2}$ sprayer & Tractor plot sprayer \\
\hline Pressure $(\mathbf{p s i})$ & & $38 \mathrm{psi}$ \\
\hline Nozzle type & $.075 \mathrm{microtube}$ & $8002 \mathrm{TwinJ} \mathrm{J}$ \\
\hline Volume $(\mathrm{gal} / \mathrm{A})$ & $5 \mathrm{gal} / \mathrm{A}$ & $19.88 \mathrm{gal} / \mathrm{A}$ \\
\hline Surfactant & & NIS $0.125 \% \mathrm{v} / \mathrm{v}$ \\
\hline
\end{tabular}

\section{APPLICATION SCHEDULE:}

\begin{tabular}{|l|l|}
\hline A & In-furrow (at planting) \\
\hline B & Pegging (49 DAP) \\
\hline C & R3 (63 DAP) \\
\hline D & R3 + 2 weeks (77 DAP) \\
\hline E & R3 + 4 weeks (91 DAP) \\
\hline F & R3 + 6 weeks (105 DAP) \\
\hline G & R3 + 8 weeks (119 DAP) \\
\hline
\end{tabular}

\section{TREATMENTS:}

\begin{tabular}{|l|l|l|l|l|}
\hline Trt \# & Product and formulation & Rate/A & Appl. timing & Appl. date \\
\hline 1 & Admire Pro 4.6 SC & $8.5 \mathrm{fl} \mathrm{oz}$ & & \\
& + Proline 480 SC & $5.7 \mathrm{fl} \mathrm{oz}$ & A & $16 \mathrm{May}$ \\
\hline 2 & Admire Pro 4.6 SC & $8.5 \mathrm{fl} \mathrm{oz}$ & & \\
& + Proline 480 SC & $5.7 \mathrm{fl} \mathrm{oz}$ & A & $16 \mathrm{May}$ \\
& Elatus 45 WG & $9.5 \mathrm{oz} \mathrm{wt}$ & & \\
& + Miravis 1.67 SC & $3.4 \mathrm{fl} \mathrm{oz}$ & $\mathrm{CE}$ & $17 \mathrm{Jul}, 13 \mathrm{Aug}$ \\
& Bravo Weather Stik & $24 \mathrm{fl} \mathrm{oz}$ & $\mathrm{G}$ & $14 \mathrm{Sep}$ \\
\hline 3 & Admire Pro 4.6 SC & $8.5 \mathrm{fl} \mathrm{oz}$ & & \\
& + Proline 480 SC & $5.7 \mathrm{fl} \mathrm{oz}$ & A & $16 \mathrm{May}$ \\
& Elatus 45 WG & $9.5 \mathrm{oz} \mathrm{wt}$ & & \\
& + Miravis 1.67 SC & $3.4 \mathrm{fl} \mathrm{oz}$ & DF & $31 \mathrm{Jul}, 28 \mathrm{Aug}$ \\
& Bravo Weather Stik & $24 \mathrm{fl} \mathrm{oz}$ & $\mathrm{G}$ & $14 \mathrm{Sep}$ \\
\hline
\end{tabular}




\begin{tabular}{|c|c|c|c|c|}
\hline 4 & $\begin{array}{l}\text { Admire Pro 4.6SC } \\
\text { + Proline 480 SC } \\
\text { Miravis 1.67 SC } \\
\text { + Tebuconazole 3.6 F } \\
\text { Bravo Weather Stik }\end{array}$ & $\begin{array}{l}8.5 \mathrm{fl} \mathrm{oz} \\
5.7 \mathrm{fl} \mathrm{oz} \\
3.4 \mathrm{fl} \mathrm{oz} \\
7.2 \mathrm{fl} \mathrm{oz} \\
24 \mathrm{fl} \mathrm{oz}\end{array}$ & $\begin{array}{l}\text { A } \\
\mathrm{CE} \\
\mathrm{G}\end{array}$ & $\begin{array}{l}16 \text { May } \\
17 \text { Jul, } 13 \text { Aug } \\
14 \text { Sep }\end{array}$ \\
\hline 5 & $\begin{array}{l}\text { Admire Pro 4.6 SC } \\
\text { + Proline 480 SC } \\
\text { Miravis 1.67 SC } \\
\text { Elatus 45 WG } \\
\text { Bravo Weather Stik } \\
\end{array}$ & $\begin{array}{l}8.5 \mathrm{fl} \mathrm{oz} \\
5.7 \mathrm{fl} \mathrm{oz} \\
3.4 \mathrm{fl} \mathrm{oz} \\
9.5 \mathrm{oz} \mathrm{wt} \\
24 \mathrm{fl} \mathrm{oz} \\
\end{array}$ & $\begin{array}{l}\text { A } \\
\text { CE } \\
\text { DF } \\
\text { G } \\
\end{array}$ & $\begin{array}{l}16 \text { May } \\
17 \text { Jul, } 13 \text { Aug } \\
31 \text { Jul, } 28 \text { Aug } \\
14 \text { Sep }\end{array}$ \\
\hline 6 & $\begin{array}{l}\text { Admire Pro 4.6 SC } \\
\text { + Proline 480 SC } \\
\text { Domark 230 ME } \\
\text { + Bravo Weather Stik } \\
\text { Bravo Weather Stik } \\
\text { + Tebuconazole 3.6 F } \\
\text { Bravo Weather Stik }\end{array}$ & $\begin{array}{l}8.5 \mathrm{fl} \mathrm{oz} \\
5.7 \mathrm{fl} \mathrm{oz} \\
2.5 \mathrm{fl} \mathrm{oz} \\
16 \mathrm{fl} \mathrm{oz} \\
24 \mathrm{fl} \mathrm{oz} \\
7.2 \mathrm{fl} \mathrm{oz} \\
24 \mathrm{fl} \mathrm{oz}\end{array}$ & $\begin{array}{l}\text { A } \\
\text { CE } \\
\text { DF } \\
\text { G }\end{array}$ & $\begin{array}{l}16 \text { May } \\
17 \text { Jul, } 13 \text { Aug } \\
\text { 31 Jul, } 28 \text { Aug } \\
14 \text { Sep }\end{array}$ \\
\hline 7 & $\begin{array}{l}\text { Admire Pro 4.6 SC } \\
+ \text { Proline } 480 \mathrm{SC} \\
\text { Miravis 1.67 SC } \\
\text { Omega 500 F } \\
\text { Bravo Weather Stik }\end{array}$ & $\begin{array}{l}8.5 \mathrm{fl} \mathrm{oz} \\
5.7 \mathrm{fl} \mathrm{oz} \\
3.4 \mathrm{fl} \mathrm{oz} \\
24 \mathrm{fl} \mathrm{oz} \\
24 \mathrm{fl} \mathrm{oz}\end{array}$ & $\begin{array}{l}\text { A } \\
\text { CE } \\
\text { DF } \\
\text { G }\end{array}$ & $\begin{array}{l}16 \text { May } \\
17 \text { Jul, } 13 \text { Aug } \\
31 \text { Jul, } 28 \text { Aug } \\
14 \text { Sep }\end{array}$ \\
\hline 8 & $\begin{array}{l}\text { Admire Pro 4.6 SC } \\
+ \text { + Proline } 480 \text { SC } \\
\text { Elatus 45 WG } \\
\text { Bravo Weather Stik } \\
+ \text { Tebuconazole 3.6 F } \\
\text { Bravo Weather Stik }\end{array}$ & $\begin{array}{l}8.5 \mathrm{fl} \mathrm{oz} \\
5.7 \mathrm{fl} \mathrm{oz} \\
9.5 \mathrm{oz} / \mathrm{wt} \\
24 \mathrm{fl} \mathrm{oz} \\
7.2 \mathrm{fl} \mathrm{oz} \\
24 \mathrm{fl} \mathrm{oz} \\
\end{array}$ & $\begin{array}{l}\mathrm{A} \\
\mathrm{CE} \\
\mathrm{DF} \\
\mathrm{G}\end{array}$ & $\begin{array}{l}16 \text { May } \\
17 \text { Jul, } 13 \text { Aug } \\
31 \text { Jul, } 28 \text { Aug } \\
14 \text { Sep }\end{array}$ \\
\hline 9 & $\begin{array}{l}\text { Admire Pro 4.6 SC } \\
\text { + Proline 480 SC } \\
\text { Priaxor 4.17 SC } \\
\text { Bravo Weather Stik } \\
\text { + Tebuconazole } 3.6 \mathrm{~F} \\
\text { Bravo Weather Stik }\end{array}$ & $\begin{array}{l}8.5 \mathrm{fl} \mathrm{oz} \\
5.7 \mathrm{fl} \mathrm{oz} \\
8 \mathrm{fl} \mathrm{oz} \\
24 \mathrm{fl} \mathrm{oz} \\
7.2 \mathrm{fl} \mathrm{oz} \\
24 \mathrm{fl} \mathrm{oz} \\
\end{array}$ & $\begin{array}{l}\mathrm{A} \\
\mathrm{CE} \\
\mathrm{DF} \\
\mathrm{G}\end{array}$ & $\begin{array}{l}16 \text { May } \\
17 \text { Jul, } 13 \text { Aug } \\
31 \text { Jul, } 28 \text { Aug }\end{array}$ \\
\hline 10 & $\begin{array}{l}\text { Admire Pro 4.6 SC } \\
\text { + Proline 480 SC } \\
\text { Provost Silver 3.52 SC } \\
\text { Bravo Weather Stik } \\
\text { + Tebuconazole } 3.6 \mathrm{~F} \\
\text { Bravo Weather Stik }\end{array}$ & $\begin{array}{l}8.5 \mathrm{fl} \mathrm{oz} \\
5.7 \mathrm{fl} \mathrm{oz} \\
13 \mathrm{fl} \mathrm{oz} \\
24 \mathrm{fl} \mathrm{oz} \\
7.2 \mathrm{fl} \mathrm{oz} \\
24 \mathrm{fl} \mathrm{oz} \\
\end{array}$ & $\begin{array}{l}\text { A } \\
\text { CE } \\
\text { DF } \\
\text { G }\end{array}$ & $\begin{array}{l}16 \text { May } \\
17 \text { Jul, } 13 \text { Aug } \\
31 \text { Jul, } 28 \text { Aug } \\
14 \text { Sep }\end{array}$ \\
\hline 11 & $\begin{array}{l}\text { Admire Pro 4.6 SC } \\
\text { + Proline 480 SC } \\
\text { Lucento 4.17 SC } \\
\text { Bravo Weather Stik } \\
\text { + Tebuconazole } 3.6 \mathrm{~F} \\
\text { Bravo Weather Stik }\end{array}$ & $\begin{array}{l}8.5 \mathrm{fl} \mathrm{oz} \\
5.7 \mathrm{fl} \mathrm{oz} \\
5.5 \mathrm{fl} \mathrm{oz} \\
24 \mathrm{fl} \mathrm{oz} \\
7.2 \mathrm{fl} \mathrm{oz} \\
24 \mathrm{fl} \mathrm{oz} \\
\end{array}$ & $\begin{array}{l}\mathrm{A} \\
\mathrm{CE} \\
\mathrm{DF} \\
\mathrm{G}\end{array}$ & $\begin{array}{l}16 \text { May } \\
17 \text { Jul, } 13 \text { Aug } \\
31 \text { Jul, } 28 \text { Aug } \\
14 \text { Sep }\end{array}$ \\
\hline 12 & $\begin{array}{l}\text { Admire Pro 4.6 SC } \\
+ \text { Proline } 480 \text { SC } \\
\text { Revytek SC } \\
\text { Bravo Weather Stik } \\
+ \text { Tebuconazole } 3.6 \text { F } \\
\text { Bravo Weather Stik }\end{array}$ & $\begin{array}{l}8.5 \mathrm{fl} \mathrm{oz} \\
5.7 \mathrm{fl} \mathrm{oz} \\
12 \mathrm{fl} \mathrm{oz} \\
24 \mathrm{fl} \mathrm{oz} \\
7.2 \mathrm{fl} \mathrm{oz} \\
24 \mathrm{fl} \mathrm{oz} \\
\end{array}$ & $\begin{array}{l}\mathrm{A} \\
\mathrm{CE} \\
\mathrm{DF} \\
\mathrm{G}\end{array}$ & $\begin{array}{l}16 \text { May } \\
17 \text { Jul, } 13 \text { Aug } \\
31 \text { Jul, } 28 \text { Aug } \\
14 \text { Sep }\end{array}$ \\
\hline
\end{tabular}




\begin{tabular}{|c|c|c|c|c|}
\hline 13 & $\begin{array}{l}\text { Admire Pro 4.6 SC } \\
\text { + Proline } 480 \text { SC } \\
\text { Excalia SC } \\
\text { Bravo Weather Stik } \\
\text { + Tebuconazole 3.6 F } \\
\text { Bravo Weather Stik } \\
\end{array}$ & $\begin{array}{l}8.5 \mathrm{fl} \mathrm{oz} \\
5.7 \mathrm{fl} \mathrm{oz} \\
2.65 \mathrm{fl} \mathrm{oz} \\
24 \mathrm{fl} \mathrm{oz} \\
7.2 \mathrm{fl} \mathrm{oz} \\
24 \mathrm{fl} \mathrm{oz} \\
\end{array}$ & $\begin{array}{l}\text { A } \\
\text { CE } \\
\text { DF } \\
\text { G }\end{array}$ & $\begin{array}{l}16 \text { May } \\
17 \text { Jul, } 13 \text { Aug } \\
31 \text { Jul, } 28 \text { Aug } \\
14 \text { Sep }\end{array}$ \\
\hline 14 & $\begin{array}{l}\text { Admire Pro 4.6 SC } \\
\text { + Velum Prime 500 SC } \\
\text { Absolute 500 SC } \\
\text { Propulse 400 SC } \\
\text { + Elatus 45 WG } \\
\text { Bravo Weather Stik } \\
\text { Provost Silver 3.52 SC }\end{array}$ & $\begin{array}{l}8.5 \mathrm{fl} \mathrm{oz} \\
6.5 \mathrm{fl} \mathrm{oz} \\
3.5 \mathrm{fl} \mathrm{oz} \\
13.6 \mathrm{fl} \mathrm{oz} \\
7.3 \mathrm{wt} \mathrm{oz} \\
24 \mathrm{fl} \mathrm{oz} \\
13 \mathrm{fl} \mathrm{oz}\end{array}$ & $\begin{array}{l}\text { A } \\
\text { C } \\
\text { D } \\
\text { EG } \\
\text { F }\end{array}$ & $\begin{array}{l}16 \text { May } \\
17 \text { Jul } \\
31 \text { Jul } \\
13 \text { Aug, 14 Sep } \\
28 \text { Aug }\end{array}$ \\
\hline 15 & $\begin{array}{l}\text { Admire Pro 4.6 SC } \\
\text { + Velum Prime 500 SC } \\
\text { Provost Silver 3.52 SC } \\
\text { Bravo Weather Stik } \\
\text { + Tebuconazole 3.6 F } \\
\text { Bravo Weather Stik }\end{array}$ & $\begin{array}{l}8.5 \mathrm{fl} \mathrm{oz} \\
15.84 \mathrm{fl} \mathrm{oz} \\
13 \mathrm{fl} \mathrm{oz} \\
24 \mathrm{fl} \mathrm{oz} \\
7.2 \mathrm{fl} \mathrm{oz} \\
24 \mathrm{fl} \mathrm{oz} \\
\end{array}$ & $\begin{array}{l}\text { A } \\
\text { CE } \\
\text { DF } \\
\text { G }\end{array}$ & $\begin{array}{l}16 \text { May } \\
17 \text { Jul, } 13 \text { Aug } \\
31 \text { Jul, } 28 \text { Aug } \\
14 \text { Sep }\end{array}$ \\
\hline 16 & $\begin{array}{l}\text { Admire Pro 4.6 SC } \\
\text { + Velum Prime 500 SC } \\
\text { Propulse 400 SC } \\
\text { Provost Silver 3.52 SC } \\
\text { Bravo Weather Stik } \\
\text { + Tebuconazole 3.6 F } \\
\text { Bravo Weather Stik } \\
\end{array}$ & $\begin{array}{l}8.5 \mathrm{fl} \mathrm{oz} \\
15.84 \mathrm{fl} \mathrm{oz} \\
13.6 \mathrm{fl} \mathrm{oz} \\
13 \mathrm{fl} \mathrm{oz} \\
24 \mathrm{fl} \mathrm{oz} \\
7.2 \mathrm{fl} \mathrm{oz} \\
24 \mathrm{fl} \mathrm{oz} \\
\end{array}$ & $\begin{array}{l}\text { A } \\
\text { B } \\
\text { CE } \\
\text { DF } \\
\text { G }\end{array}$ & $\begin{array}{l}16 \text { May } \\
1 \text { Jul } \\
17 \text { Jul, } 13 \text { Aug } \\
\text { 31 Jul, } 28 \text { Aug } \\
14 \text { Sep }\end{array}$ \\
\hline 17 & $\begin{array}{l}\text { Admire Pro 4.6 SC } \\
\text { + Proline 480 SC } \\
\text { Propulse 400 SC } \\
\text { Provost Silver } 3.52 \text { SC } \\
\text { Bravo Weather Stik } \\
\text { + Tebuconazole } 3.6 \text { F } \\
\text { Bravo Weather Stik }\end{array}$ & $\begin{array}{l}8.5 \mathrm{fl} \mathrm{oz} \\
5.7 \mathrm{fl} \mathrm{oz} \\
13.6 \mathrm{fl} \mathrm{oz} \\
13 \mathrm{fl} \mathrm{oz} \\
24 \mathrm{fl} \mathrm{oz} \\
7.2 \mathrm{fl} \mathrm{oz} \\
24 \mathrm{fl} \mathrm{oz}\end{array}$ & $\begin{array}{l}\text { A } \\
\text { B } \\
\text { CE } \\
\text { DF } \\
\text { G }\end{array}$ & $\begin{array}{l}16 \text { May } \\
1 \text { Jul } \\
17 \text { Jul, } 13 \text { Aug } \\
31 \text { Jul, } 28 \text { Aug } \\
14 \text { Sep }\end{array}$ \\
\hline
\end{tabular}

SOIL TYPE: Kenansville loamy fine sand

SOIL FERTILITY REPORT (Jan 2020):

\begin{tabular}{|l|l|l|l|l|l|l|l|l|l|}
\hline $\mathbf{p H}$ & $\mathbf{P}(\mathbf{l b} / \mathbf{A})$ & $\mathbf{K}(\mathbf{l b} / \mathbf{A})$ & $\mathbf{C a}(\mathbf{l b} / \mathbf{A})$ & $\mathbf{M g}(\mathbf{l b} / \mathbf{A})$ & $\mathbf{Z n}(\mathbf{p p m})$ & $\mathbf{M n}(\mathbf{p p m})$ & $\mathbf{C u}(\mathbf{p p m})$ & $\mathbf{F e}(\mathbf{p p m})$ & $\mathbf{B}(\mathbf{p p m})$ \\
\hline 6.4 & 72 & 86 & 574 & 68 & 0.6 & 3.3 & 0.2 & 16.0 & 0.1 \\
\hline
\end{tabular}

MAINTENANCE CHEMICAL PROGRAMS:

\begin{tabular}{|l|l|}
\hline Fertilizer & Standard \\
\hline Herbicides & Standard \\
\hline Insecticides & Standard \\
\hline Fungicides & None except treatments \\
\hline Nematicides & None except treatments \\
\hline
\end{tabular}


MAINTENANCE CHEMICAL APPLICATIONS:

\begin{tabular}{|l|l|l|l|}
\hline Date & Type and target & Product and formulation & Rate/A \\
\hline $5 / 17 / 20$ & Herbicide & Strongarm & $0.45 \mathrm{fl} \mathrm{oz}$ \\
\hline $5 / 17 / 20$ & Herbicide & Dual II MAGNUM & $1.5 \mathrm{pt}$ \\
\hline $5 / 17 / 20$ & Herbicide & Prowl H2O & $1.0 \mathrm{pt}$ \\
\hline $5 / 17 / 20$ & Fertility & Boron & $1.0 \mathrm{qt}$ \\
\hline $6 / 4 / 20$ & Insecticide & Orthene $75 \mathrm{~S}$ & $12 \mathrm{oz}$ \\
\hline $6 / 25 / 20$ & Herbicide & Basagran & $1.5 \mathrm{pt}$ \\
\hline $6 / 25 / 20$ & Herbicide & Fusilade DX & $1 \mathrm{pt}$ \\
\hline $7 / 7 / 20$ & Herbicide & Storm & $1.5 \mathrm{pt}$ \\
\hline $7 / 7 / 20$ & Herbicide & Basagran & $1 \mathrm{pt}$ \\
\hline $7 / 13 / 20$ & Fertility & Manganese & $2 \mathrm{qt}$ \\
\hline $7 / 13 / 20$ & Insecticide & Danitol 2.4 EC & $8 \mathrm{fl} \mathrm{oz}$ \\
\hline $7 / 17 / 20$ & Fertility & Landplaster & $1500 \mathrm{lb}$ \\
\hline $7 / 24 / 20$ & Insecticide & Danitol 2.4 EC & $8 \mathrm{fl} \mathrm{oz}$ \\
\hline $8 / 6 / 20$ & Herbicide & Tide 2 EC $($ Clethodim) & $16 \mathrm{fl} \mathrm{oz}$ \\
\hline
\end{tabular}


Table 1. Effect of fungicide treatment on disease incidence in peanut (PTLFSPOT420, Suffolk, VA 2020).

\begin{tabular}{|c|c|c|c|c|c|}
\hline \multirow{2}{*}{$\begin{array}{l}\text { Treatment, rate/A } \\
\text { and application timing/date }{ }^{\mathrm{z}}\end{array}$} & \multicolumn{4}{|c|}{ Leaf spot $(1-10)^{y}$} & \multirow{2}{*}{$\begin{array}{l}\% \text { leaf } \\
\text { spot }^{w} \\
\text { 8 Oct }\end{array}$} \\
\hline & 26 Aug & 13 Sep & 28 Sep & AUDPC $^{x}$ & \\
\hline $\begin{array}{ll}\text { 1. } & \text { Admire Pro 4.6 SC } 8.5 \mathrm{fl} \mathrm{oz} \\
& + \text { Proline 480 SC } 5.7 \mathrm{fl} \mathrm{oz} \mathrm{(F)}\end{array}$ & 1.8 & $4.0 \mathrm{a}$ & $6.3 \mathrm{a}$ & $128.6 \mathrm{a}$ & $97.5 \mathrm{a}$ \\
\hline $\begin{array}{ll}\text { 2. } & \text { Admire Pro 4.6 SC } 8.5 \mathrm{fl} \mathrm{oz} \\
& + \text { Proline } 480 \mathrm{SC} 5.7 \mathrm{fl} \mathrm{oz}(\mathrm{F}) \\
& \text { Elatus 45 WG } 9.5 \mathrm{oz} / \mathrm{wt} \\
& + \text { Miravis } 1.67 \mathrm{SC} 3.4 \mathrm{fl} \mathrm{oz}(7 / 17,8 / 13) \\
& \text { Bravo Weather Stik } 24 \mathrm{fl} \mathrm{oz}(9 / 14) \\
\end{array}$ & 2.5 & $1.0 \mathrm{~d}$ & $1.8 \mathrm{~b}$ & $52.1 \mathrm{bc}$ & $57.5 \mathrm{~b}$ \\
\hline $\begin{array}{ll}\text { 3. } & \text { Admire Pro 4.6 SC } 8.5 \mathrm{fl} \mathrm{oz} \\
+ \text { Proline } 480 \mathrm{SC} 5.7 \mathrm{fl} \mathrm{oz}(\mathrm{F}) \\
\text { Elatus 45 WG } 9.5 \mathrm{oz} / \mathrm{wt} \\
\text { + Miravis } 1.67 \text { SC } 3.4 \mathrm{fl} \mathrm{oz}(7 / 31,8 / 28) \\
\text { Bravo Weather Stik } 24 \mathrm{fl} \mathrm{oz}(9 / 14)\end{array}$ & 1.8 & $1.0 \mathrm{~d}$ & $1.8 \mathrm{~b}$ & $45.4 \mathrm{bc}$ & $1.0 \mathrm{e}$ \\
\hline $\begin{array}{l}\text { 4. } \\
\text { Admire Pro 4.6 SC } 8.5 \mathrm{fl} \mathrm{oz} \\
\text { + Proline 480 SC } 5.7 \mathrm{fl} \mathrm{oz}(\mathrm{F}) \\
\text { Miravis } 1.67 \mathrm{SC} 3.4 \mathrm{fl} \mathrm{oz} \\
\text { + Tebuconazole } 3.6 \mathrm{~F} 7.2 \mathrm{fl} \mathrm{oz}(7 / 31,8 / 28) \\
\text { Bravo Weather Stik } 24 \mathrm{fl} \mathrm{oz}(9 / 14)\end{array}$ & 2.0 & $1.3 \mathrm{~cd}$ & $1.8 \mathrm{~b}$ & $51.8 \mathrm{bc}$ & $40.0 \mathrm{c}$ \\
\hline $\begin{array}{ll}\text { 5. } & \text { Admire Pro 4.6 SC } 8.5 \mathrm{fl} \mathrm{oz} \\
\text { + Proline 480 SC } 5.7 \mathrm{fl} \mathrm{oz}(\mathrm{F}) \\
\text { Miravis } 1.67 \mathrm{SC} 3.4 \mathrm{fl} \mathrm{oz}(7 / 17,8 / 13) \\
\text { Elatus 45 WG } 9.5 \mathrm{oz} / \mathrm{wt}(7 / 31,8 / 28) \\
\text { Bravo Weather Stik } 24 \mathrm{fl} \mathrm{oz}(9 / 14) \\
\end{array}$ & 1.8 & $1.0 \mathrm{~d}$ & $1.0 \mathrm{~b}$ & $39.8 \mathrm{c}$ & $5.3 \mathrm{e}$ \\
\hline $\begin{array}{ll}\text { 6. } & \text { Admire Pro 4.6 SC } 8.5 \mathrm{fl} \mathrm{oz} \\
\text { + Proline 480 SC } 5.7 \mathrm{fl} \mathrm{oz} \mathrm{(F)} \\
\text { Domark 230 ME } 2.5 \mathrm{fl} \mathrm{oz} \\
\text { + Bravo Weather Stik } 16 \mathrm{fl} \mathrm{oz}(7 / 17,8 / 13) \\
\text { Bravo Weather Stik } 24 \mathrm{fl} \mathrm{oz} \\
\text { + Tebuconazole 3.6 F } 7.2 \mathrm{fl} \mathrm{oz}(7 / 31,8 / 28) \\
\text { Bravo Weather Stik } 24 \mathrm{fl} \mathrm{oz}(9 / 14) \\
\end{array}$ & 1.8 & $1.3 \mathrm{~cd}$ & $1.3 \mathrm{~b}$ & $45.8 \mathrm{bc}$ & $12.5 \mathrm{de}$ \\
\hline $\begin{array}{ll}\text { 7. } & \text { Admire Pro 4.6 SC } 8.5 \mathrm{fl} \mathrm{oz} \\
\text { + Proline 480 SC } 5.7 \mathrm{fl} \mathrm{oz}(\mathrm{F}) \\
\text { Miravis } 1.67 \mathrm{SC} 3.4 \mathrm{fl} \mathrm{oz}(7 / 17,8 / 13) \\
\text { Omega 500 F } 24 \mathrm{fl} \mathrm{oz}(7 / 31,8 / 28) \\
\text { Bravo Weather Stik } 24 \mathrm{fl} \mathrm{oz}(9 / 14) \\
\end{array}$ & 2.0 & $1.0 \mathrm{~d}$ & $1.3 \mathrm{~b}$ & $43.9 \mathrm{c}$ & $10.0 \mathrm{de}$ \\
\hline $\begin{array}{l}\text { 8. } \\
\text { + Proline } 480 \text { SC } 5.7 \mathrm{fl} \mathrm{oz}(\mathrm{F}) \\
\text { Elatus } 45 \text { WG } 9.5 \mathrm{oz} / \mathrm{wt}(7 / 17,8 / 13) \\
\text { Bravo Weather Stik } 24 \mathrm{fl} \mathrm{oz} \\
\text { + Tebuconazole } 3.6 \mathrm{~F} 7.2 \mathrm{fl} \mathrm{oz}(7 / 31,8 / 28) \\
\text { Bravo Weather Stik } 24 \mathrm{fl} \mathrm{oz}(9 / 14)\end{array}$ & 1.8 & $2.0 \mathrm{bc}$ & $1.8 \mathrm{~b}$ & $61.9 \mathrm{~b}$ & $27.5 \mathrm{~cd}$ \\
\hline $\begin{array}{l}\text { 9. } \\
\text { + Proline Pro 4.6 SC } 8.5 \mathrm{fl} \mathrm{oz} \\
\text { Priaxor 4.17 SC } 5.7 \mathrm{fl} \mathrm{oz}(\mathrm{F}) \\
\text { Bravo Weather Stik } 24 \mathrm{fl} \mathrm{oz} \\
\text { + Tebuconazole } 3.6 \mathrm{~F} 7.2 \mathrm{fl} \mathrm{oz}(7 / 31,8 / 28) \\
\text { Bravo Weather Stik } 24 \mathrm{fl} \mathrm{oz}(9 / 14) \\
\end{array}$ & 1.8 & $1.5 \mathrm{~b}-\mathrm{d}$ & $1.3 \mathrm{~b}$ & $49.9 \mathrm{bc}$ & $20.0 \mathrm{de}$ \\
\hline $\begin{array}{l}\text { 10. } \text { Admire Pro 4.6 SC } 8.5 \mathrm{fl} \mathrm{oz} \\
\text { + Proline 480 SC 5.7 fl oz (F) } \\
\text { Provost Silver 3.52 SC } 13 \mathrm{fl} \mathrm{oz}(7 / 17,8 / 13) \\
\text { Bravo Weather Stik } 24 \mathrm{fl} \mathrm{oz} \\
\text { + Tebuconazole } 3.6 \mathrm{~F} 7.2 \mathrm{fl} \mathrm{oz}(7 / 31,8 / 28) \\
\text { Bravo Weather Stik } 24 \mathrm{fl} \mathrm{oz}(9 / 14) \\
\end{array}$ & 1.5 & $1.5 \mathrm{~b}-\mathrm{d}$ & $1.0 \mathrm{~b}$ & $45.8 \mathrm{bc}$ & $18.8 \mathrm{de}$ \\
\hline $\begin{array}{l}\text { 11. Admire Pro 4.6 SC } 8.5 \mathrm{fl} \mathrm{oz} \\
\text { + Proline 480 SC } 5.7 \mathrm{fl} \mathrm{oz}(\mathrm{F}) \\
\text { Lucento 4.17 SC } 5.5 \mathrm{fl} \mathrm{oz}(7 / 13,8 / 13) \\
\text { Bravo Weather Stik } 24 \mathrm{fl} \mathrm{oz} \\
\text { + Tebuconazole } 3.6 \mathrm{~F} 7.2 \mathrm{fl} \mathrm{oz}(7 / 31,8 / 28) \\
\text { Bravo Weather Stik } 24 \mathrm{fl} \mathrm{oz}(9 / 14)\end{array}$ & 1.3 & $1.5 \mathrm{~b}-\mathrm{d}$ & $1.3 \mathrm{~b}$ & $45.4 \mathrm{bc}$ & $13.8 \mathrm{de}$ \\
\hline
\end{tabular}




\begin{tabular}{|c|c|c|c|c|c|}
\hline $\begin{array}{l}\text { 12. Admire Pro 4.6 SC } 8.5 \mathrm{fl} \mathrm{oz} \\
\text { + Proline 480 SC } 5.7 \mathrm{fl} \mathrm{oz} \mathrm{(F)} \\
\text { Revytek SC } 12 \mathrm{fl} \mathrm{oz}(7 / 17,8 / 13) \\
\text { Bravo Weather Stik } 24 \mathrm{fl} \mathrm{oz} \\
\text { + Tebuconazole } 3.6 \mathrm{~F} 7.2 \mathrm{fl} \mathrm{oz}(7 / 31,8 / 28) \\
\text { Bravo Weather Stik } 24 \mathrm{fl} \mathrm{oz}(9 / 14)\end{array}$ & 1.8 & $1.0 \mathrm{~d}$ & $1.0 \mathrm{~b}$ & $39.8 \mathrm{c}$ & $12.5 \mathrm{de}$ \\
\hline $\begin{array}{l}\text { 13. Admire Pro 4.6 SC } 8.5 \mathrm{fl} \mathrm{oz} \\
+ \text { Proline 480 SC } 5.7 \mathrm{fl} \mathrm{oz} \mathrm{(F)} \\
\text { Excalia SC 2.65 fl oz }(7 / 17,8 / 13) \\
\text { Bravo Weather Stik } 24 \mathrm{fl} \mathrm{oz} \\
\text { + Tebuconazole 3.6 F } 7.2 \mathrm{fl} \mathrm{oz}(7 / 31,8 / 28) \\
\text { Bravo Weather Stik } 24 \mathrm{fl} \mathrm{oz}(9 / 14) \\
\end{array}$ & 1.5 & $2.3 \mathrm{~b}$ & $1.5 \mathrm{~b}$ & $61.9 \mathrm{~b}$ & $40.0 \mathrm{c}$ \\
\hline $\begin{array}{l}\text { 14. Admire Pro 4.6 SC } 8.5 \mathrm{fl} \mathrm{oz} \\
\text { + Velum Prime } 500 \mathrm{SC} 15.84 \mathrm{fl} \mathrm{oz}(\mathrm{F}) \\
\text { Absolute 500 SC } 3.5 \mathrm{fl} \mathrm{oz}(7 / 17) \\
\text { Propulse 400 SC } 13.6 \mathrm{fl} \mathrm{oz} \\
\text { + Elatus } 45 \mathrm{WG} 7.3 \mathrm{wt} / \mathrm{oz}(7 / 31) \\
\text { Bravo Weather Stik } 24 \mathrm{fl} \mathrm{oz}(8 / 13,9 / 14) \\
\text { Provost Silver } 3.52 \mathrm{SC} 13 \mathrm{fl} \mathrm{oz}(8 / 28) \\
\end{array}$ & 1.5 & $1.5 \mathrm{~b}-\mathrm{d}$ & $1.3 \mathrm{~b}$ & $47.6 \mathrm{bc}$ & $23.8 \mathrm{~cd}$ \\
\hline $\begin{array}{l}\text { 15. Admire Pro 4.6 SC } 8.5 \mathrm{fl} \mathrm{oz} \\
+ \text { Velum Prime 500 SC } 6.5 \mathrm{fl} \mathrm{oz}(\mathrm{F}) \\
\text { Provost Silver } 3.52 \mathrm{SC} 13 \mathrm{fl} \mathrm{oz}(7 / 17,8 / 13) \\
\text { Bravo Weather Stik } 24 \mathrm{fl} \mathrm{oz} \\
+ \text { Tebuconazole } 3.6 \mathrm{~F} 7.2 \mathrm{fl} \mathrm{oz}(7 / 31,8 / 28) \\
\text { Bravo Weather Stik } 24 \mathrm{fl} \mathrm{oz}(9 / 14)\end{array}$ & 1.8 & $1.3 \mathrm{~cd}$ & $1.0 \mathrm{~b}$ & $43.9 \mathrm{c}$ & $12.5 \mathrm{de}$ \\
\hline $\begin{array}{l}\text { 16. Admire Pro 4.6 SC } 8.5 \mathrm{fl} \mathrm{oz} \\
+ \text { Velum Prime 500 SC } 15.84 \mathrm{fl} \mathrm{oz}(\mathrm{F}) \\
\text { Propulse 400 SC } 13.6 \mathrm{fl} \mathrm{oz} \\
\text { Provost Silver 3.52 SC } 13 \mathrm{fl} \mathrm{oz}(7 / 13,8 / 13) \\
\text { Bravo Weather Stik } 24 \mathrm{fl} \mathrm{oz} \\
+ \text { Tebuconazole } 3.6 \mathrm{~F} 7.2 \mathrm{fl} \mathrm{oz}(7 / 31,8 / 28) \\
\text { Bravo Weather Stik } 24 \mathrm{fl} \mathrm{oz}(9 / 14)\end{array}$ & 1.8 & $1.8 \mathrm{~b}-\mathrm{d}$ & $1.0 \mathrm{~b}$ & $52.1 \mathrm{bc}$ & $10.3 \mathrm{de}$ \\
\hline $\begin{array}{l}\text { 17. Admire Pro 4.6 SC } 8.5 \mathrm{fl} \mathrm{oz} \\
\text { + Proline 480 SC } 5.7 \mathrm{fl} \mathrm{oz} \mathrm{(F)} \\
\text { Propulse 400 SC } 13.6 \mathrm{fl} \mathrm{oz} \\
\text { Provost Silver } 3.52 \text { SC } 13 \mathrm{fl} \mathrm{oz} 7 / 17,8 / 13) \\
\text { Bravo Weather Stik } 24 \mathrm{fl} \mathrm{oz} \\
\text { + Tebuconazole } 3.6 \mathrm{~F} 7.2 \mathrm{fl} \mathrm{oz}(7 / 31,8 / 28) \\
\text { Bravo Weather Stik } 24 \mathrm{fl} \mathrm{oz}(9 / 14) \\
\end{array}$ & 1.8 & $1.3 \mathrm{~cd}$ & $1.5 \mathrm{~b}$ & $47.6 \mathrm{bc}$ & $8.8 \mathrm{de}$ \\
\hline$P(\mathrm{~F})$ & 0.28 & $<0.0001$ & $<0.0001$ & $<0.0001$ & $<0.0001$ \\
\hline LSD & N.S. & 0.77 & 0.83 & 16.96 & 16.70 \\
\hline
\end{tabular}

z (F) In-furrow (16 May); (P) broadcast at pegging (1 Jul). First fungicide for leaf spot program was applied at R3 (17 Jul) and followed by applications on two-week timings as prescribed. All foliar treatments applied with Induce $0.125 \% \mathrm{v} / \mathrm{v}$.

y Florida leaf spot scale: $1=$ no disease, $10=$ dead plants, Chiteka et al. (1988). Untreated control (Trt. 1) plots were observed for leaf spot on $7 \mathrm{Aug}$, little to no lesions observed.

$x$ AUDPC $=$ area under the disease progress curve.

${ }^{w}$ Percent of leaves in two treatment rows with symptoms of leaf spot. Means in a column followed by the same letter(s) are not significantly different according to Fisher's Protected LSD $(P=0.05)$. Arcsine transformation of percentage data was made in analysis to determine statistical significance. 
Table 2. Effect of fungicide treatment on soilborne disease and yield in peanut (PTLFSPOT420, Suffolk, VA 2020).

\begin{tabular}{|c|c|c|c|c|c|}
\hline \multirow{2}{*}{$\begin{array}{l}\text { Treatment, rate/A } \\
\text { and application timing/date }\end{array}$} & \multicolumn{4}{|c|}{ Sclerotinia blight $^{\mathbf{y}}$} & \multirow{2}{*}{$\begin{array}{c}\text { Yield } \\
(\mathbf{l b} / \mathbf{A})^{\mathrm{w}}\end{array}$} \\
\hline & 13 Sep & 28 Sep & 15 Oct & AUDPC $^{x}$ & \\
\hline $\begin{array}{ll}\text { 1. } & \text { Admire Pro 4.6 SC } 8.5 \mathrm{fl} \mathrm{oz} \\
& + \text { Proline 480 SC } 5.7 \mathrm{fl} \mathrm{oz} \mathrm{(F)}\end{array}$ & $12.8 \mathrm{ab}$ & $38.8 \mathrm{ab}$ & $45.5 \mathrm{a}$ & $1102.4 \mathrm{a}$ & $1538 \mathrm{~d}-\mathrm{f}$ \\
\hline $\begin{array}{ll}\text { 2. } & \text { Admire Pro 4.6 SC } 8.5 \mathrm{fl} \mathrm{oz} \\
& + \text { Proline } 480 \mathrm{SC} 5.7 \mathrm{fl} \mathrm{oz}(\mathrm{F}) \\
& \text { Elatus 45 WG } 9.5 \mathrm{oz} / \mathrm{wt} \\
& + \text { Miravis } 1.67 \mathrm{SC} 3.4 \mathrm{fl} \mathrm{oz}(7 / 17,8 / 13) \\
& \text { Bravo Weather Stik } 24 \mathrm{fl} \mathrm{oz}(9 / 14) \\
\end{array}$ & $1.0 \mathrm{~g}$ & $11.0 \mathrm{gh}$ & $35.3 \mathrm{~cd}$ & $483.1 \mathrm{fg}$ & $3459 \mathrm{ab}$ \\
\hline $\begin{array}{ll}\text { 3. } & \text { Admire Pro 4.6 SC } 8.5 \mathrm{fl} \mathrm{oz} \\
\text { + Proline 480 SC } 5.7 \mathrm{fl} \mathrm{oz}(\mathrm{F}) \\
\text { Elatus 45 WG 9.5 oz/wt } \\
\text { + Miravis } 1.67 \text { SC } 3.4 \mathrm{fl} \mathrm{oz}(7 / 31,8 / 28) \\
\text { Bravo Weather Stik } 24 \mathrm{fl} \mathrm{oz}(9 / 14)\end{array}$ & $4.3 \mathrm{~d}-\mathrm{g}$ & $15.8 \mathrm{fg}$ & $35.0 \mathrm{~cd}$ & $581.4 \mathrm{ef}$ & $3675 \mathrm{a}$ \\
\hline $\begin{array}{l}\text { 4. } \\
\text { Admire Pro 4.6 SC } 8.5 \mathrm{fl} \mathrm{oz} \\
\text { + Proline 480 SC } 5.7 \mathrm{fl} \mathrm{oz}(\mathrm{F}) \\
\text { Miravis 1.67 SC } 3.4 \mathrm{fl} \mathrm{oz} \\
\text { + Tebuconazole } 3.6 \mathrm{~F} 7.2 \mathrm{fl} \mathrm{oz}(7 / 31,8 / 28) \\
\text { Bravo Weather Stik } 24 \mathrm{fl} \mathrm{oz}(9 / 14)\end{array}$ & $4.8 \mathrm{~d}-\mathrm{g}$ & $22.3 \mathrm{ef}$ & $38.5 \mathrm{bc}$ & $718.9 \mathrm{de}$ & $2573 \mathrm{bc}$ \\
\hline $\begin{array}{ll}\text { 5. } & \text { Admire Pro 4.6 SC } 8.5 \mathrm{fl} \mathrm{oz} \\
\text { + Proline 480 SC } 5.7 \mathrm{fl} \mathrm{oz}(\mathrm{F}) \\
\text { Miravis } 1.67 \mathrm{SC} 3.4 \mathrm{fl} \mathrm{oz}(7 / 17,8 / 13) \\
\text { Elatus 45 WG } 9.5 \mathrm{oz} / \mathrm{wt}(7 / 31,8 / 28) \\
\text { Bravo Weather Stik } 24 \mathrm{fl} \mathrm{oz}(9 / 14) \\
\end{array}$ & $2.3 \mathrm{e}-\mathrm{g}$ & $6.5 \mathrm{gh}$ & $30.0 \mathrm{de}$ & $375.9 \mathrm{fg}$ & $4052 \mathrm{a}$ \\
\hline $\begin{array}{ll}\text { 6. } & \text { Admire Pro 4.6 SC } 8.5 \mathrm{fl} \mathrm{oz} \\
\text { + Proline 480 SC } 5.7 \mathrm{fl} \mathrm{oz} \mathrm{(F)} \\
\text { Domark 230 ME } 2.5 \mathrm{fl} \mathrm{oz} \\
\text { + Bravo Weather Stik } 16 \mathrm{fl} \mathrm{oz}(7 / 17,8 / 13) \\
\text { Bravo Weather Stik } 24 \mathrm{fl} \mathrm{oz} \\
\text { + Tebuconazole 3.6 F } 7.2 \mathrm{fl} \mathrm{oz}(7 / 31,8 / 28) \\
\text { Bravo Weather Stik } 24 \mathrm{fl} \mathrm{oz}(9 / 14) \\
\end{array}$ & $13.3 \mathrm{a}$ & $39.3 \mathrm{ab}$ & $45.0 \mathrm{a}$ & $1109.9 \mathrm{a}$ & 1247 ef \\
\hline $\begin{array}{ll}\text { 7. } & \text { Admire Pro 4.6 SC } 8.5 \mathrm{fl} \mathrm{oz} \\
\text { + Proline 480 SC } 5.7 \mathrm{fl} \mathrm{oz}(\mathrm{F}) \\
\text { Miravis } 1.67 \mathrm{SC} 3.4 \mathrm{fl} \mathrm{oz}(7 / 17,8 / 13) \\
\text { Omega 500 F } 24 \mathrm{fl} \mathrm{oz}(7 / 31,8 / 28) \\
\text { Bravo Weather Stik } 24 \mathrm{fl} \mathrm{oz}(9 / 14) \\
\end{array}$ & $1.5 \mathrm{fg}$ & $5.3 \mathrm{~h}$ & $28.0 \mathrm{e}$ & $333.3 \mathrm{~g}$ & $3825 \mathrm{a}$ \\
\hline $\begin{array}{ll}\text { 8. } & \text { Admire Pro 4.6 SC } 8.5 \mathrm{fl} \mathrm{oz} \\
\text { + Proline 480 SC } 5.7 \mathrm{fl} \mathrm{oz}(\mathrm{F}) \\
\text { Elatus 45 WG } 9.5 \mathrm{oz} / \mathrm{wt}(7 / 17,8 / 13) \\
\text { Bravo Weather Stik } 24 \mathrm{fl} \mathrm{oz} \\
\text { + Tebuconazole 3.6 F } 7.2 \mathrm{fl} \mathrm{oz}(7 / 31,8 / 28) \\
\text { Bravo Weather Stik } 24 \mathrm{fl} \mathrm{oz}(9 / 14) \\
\end{array}$ & $6.0 \mathrm{c}-\mathrm{g}$ & $23.8 \mathrm{~d}-\mathrm{f}$ & $42.0 \mathrm{ab}$ & $782.0 \mathrm{c}-\mathrm{e}$ & $2430 \mathrm{~cd}$ \\
\hline $\begin{array}{l}\text { 9. } \\
\text { + Proline Pro 4.6 SC } 8.5 \mathrm{fl} \mathrm{oz} \\
\text { Priaxor 4.17 SC } 5.7 \mathrm{fl} \mathrm{oz}(\mathrm{F}) \\
\text { Bravo Weather Stik } 24 \mathrm{fl} \mathrm{oz} \\
\text { + Tebuconazole } 3.6 \mathrm{~F} 7.2 \mathrm{fl} \mathrm{oz}(7 / 31,8 / 28) \\
\text { Bravo Weather Stik } 24 \mathrm{fl} \mathrm{oz}(9 / 14) \\
\end{array}$ & $5.0 \mathrm{~d}-\mathrm{g}$ & $26.5 \mathrm{c}-\mathrm{e}$ & $43.3 \mathrm{ab}$ & $829.1 \mathrm{~b}-\mathrm{d}$ & $2055 \mathrm{c}-\mathrm{e}$ \\
\hline $\begin{array}{l}\text { 10. } \text { Admire Pro 4.6 SC } 8.5 \mathrm{fl} \mathrm{oz} \\
\text { + Proline 480 SC 5.7 fl oz (F) } \\
\text { Provost Silver 3.52 SC } 13 \mathrm{fl} \mathrm{oz}(7 / 17,8 / 13) \\
\text { Bravo Weather Stik } 24 \mathrm{fl} \mathrm{oz} \\
\text { + Tebuconazole } 3.6 \mathrm{~F} 7.2 \mathrm{fl} \mathrm{oz}(7 / 31,8 / 28) \\
\text { Bravo Weather Stik } 24 \mathrm{fl} \mathrm{oz}(9 / 14) \\
\end{array}$ & $12.0 \mathrm{a}-\mathrm{c}$ & $37.8 \mathrm{ab}$ & $44.8 \mathrm{a}$ & $1074.4 \mathrm{a}$ & $1539 \mathrm{~d}-\mathrm{f}$ \\
\hline $\begin{array}{l}\text { 11. Admire Pro 4.6 SC } 8.5 \mathrm{fl} \mathrm{oz} \\
\text { + Proline 480 SC } 5.7 \mathrm{fl} \mathrm{oz}(\mathrm{F}) \\
\text { Lucento 4.17 SC } 5.5 \mathrm{fl} \mathrm{oz}(7 / 13,8 / 13) \\
\text { Bravo Weather Stik } 24 \mathrm{fl} \mathrm{oz} \\
\text { + Tebuconazole } 3.6 \mathrm{~F} 7.2 \mathrm{fl} \mathrm{oz}(7 / 31,8 / 28) \\
\text { Bravo Weather Stik } 24 \mathrm{fl} \mathrm{oz}(9 / 14)\end{array}$ & $9.8 \mathrm{a}-\mathrm{d}$ & $33.8 \mathrm{a}-\mathrm{c}$ & $44.5 \mathrm{ab}$ & $991.4 \mathrm{a}-\mathrm{c}$ & $1378 \mathrm{ef}$ \\
\hline
\end{tabular}




\begin{tabular}{|c|c|c|c|c|c|}
\hline $\begin{array}{l}\text { 12. } \\
\text { Admire Pro 4.6 SC } 8.5 \mathrm{fl} \mathrm{oz} \\
+ \text { Proline } 480 \text { SC } 5.7 \mathrm{fl} \mathrm{oz} \mathrm{(F)} \\
\text { Revytek SC } 12 \mathrm{fl} \mathrm{oz}(7 / 17,8 / 13) \\
\text { Bravo Weather Stik } 24 \mathrm{fl} \mathrm{oz} \\
+ \text { Tebuconazole } 3.6 \mathrm{~F} 7.2 \mathrm{fl} \mathrm{oz}(7 / 31,8 / 28) \\
\text { Bravo Weather Stik } 24 \mathrm{fl} \mathrm{oz}(9 / 14)\end{array}$ & $12.5 \mathrm{a}-\mathrm{c}$ & $34.3 \mathrm{a}-\mathrm{c}$ & $44.8 \mathrm{a}$ & $1022.1 \mathrm{ab}$ & 1354 ef \\
\hline $\begin{array}{l}\text { 13. Admire Pro 4.6 SC } 8.5 \mathrm{fl} \mathrm{oz} \\
+ \text { Proline } 480 \text { SC } 5.7 \mathrm{fl} \mathrm{oz}(\mathrm{F}) \\
\text { Excalia SC } 2.65 \mathrm{fl} \mathrm{oz}(7 / 17,8 / 13) \\
\text { Bravo Weather Stik } 24 \mathrm{fl} \mathrm{oz} \\
\text { + Tebuconazole } 3.6 \mathrm{~F} 7.2 \mathrm{fl} \mathrm{oz}(7 / 31,8 / 28) \\
\text { Bravo Weather Stik } 24 \mathrm{fl} \mathrm{oz}(9 / 14)\end{array}$ & $13.3 \mathrm{a}$ & $41.0 \mathrm{a}$ & $45.5 \mathrm{a}$ & $1142.1 \mathrm{a}$ & $954 \mathrm{f}$ \\
\hline $\begin{array}{l}\text { 14. Admire Pro 4.6 SC } 8.5 \mathrm{fl} \mathrm{oz} \\
\text { + Velum Prime } 500 \mathrm{SC} 15.84 \mathrm{fl} \mathrm{oz}(\mathrm{F}) \\
\text { Absolute 500 SC } 3.5 \mathrm{fl} \mathrm{oz}(7 / 17) \\
\text { Propulse 400 SC } 13.6 \mathrm{fl} \mathrm{oz} \\
\text { + Elatus 45 WG } 7.3 \mathrm{wt} / \mathrm{oz}(7 / 31) \\
\text { Bravo Weather Stik } 24 \mathrm{fl} \mathrm{oz}(8 / 13,9 / 14) \\
\text { Provost Silver } 3.52 \mathrm{SC} 13 \mathrm{fl} \mathrm{oz}(8 / 28) \\
\end{array}$ & $7.5 \mathrm{a}-\mathrm{g}$ & $25.5 \mathrm{c}-\mathrm{e}$ & $44.3 \mathrm{ab}$ & 840.4 b-d & $2088 \mathrm{c}-\mathrm{e}$ \\
\hline $\begin{array}{l}\text { 15. Admire Pro 4.6 SC } 8.5 \mathrm{fl} \mathrm{oz} \\
+ \text { Velum Prime 500 SC } 6.5 \mathrm{fl} \mathrm{oz}(\mathrm{F}) \\
\text { Provost Silver 3.52 SC } 13 \mathrm{fl} \mathrm{oz}(7 / 17,8 / 13) \\
\text { Bravo Weather Stik } 24 \mathrm{fl} \mathrm{oz} \\
\text { + Tebuconazole 3.6 F } 7.2 \mathrm{fl} \mathrm{oz}(7 / 31,8 / 28) \\
\text { Bravo Weather Stik } 24 \mathrm{fl} \mathrm{oz}(9 / 14)\end{array}$ & $8.0 \mathrm{a}-\mathrm{f}$ & $31.5 \mathrm{~b}-\mathrm{e}$ & $43.5 \mathrm{ab}$ & $933.8 \mathrm{a}-\mathrm{c}$ & 1992 c-e \\
\hline $\begin{array}{l}\text { 16. Admire Pro 4.6 SC } 8.5 \mathrm{fl} \mathrm{oz} \\
+ \text { Velum Prime 500 SC } 15.84 \mathrm{fl} \mathrm{oz}(\mathrm{F}) \\
\text { Propulse 400 SC } 13.6 \mathrm{fl} \mathrm{oz} \\
\text { Provost Silver 3.52 SC } 13 \mathrm{fl} \mathrm{oz}(7 / 13,8 / 13) \\
\text { Bravo Weather Stik } 24 \mathrm{fl} \mathrm{oz} \\
+ \text { Tebuconazole 3.6 F } 7.2 \mathrm{fl} \mathrm{oz}(7 / 31,8 / 28) \\
\text { Bravo Weather Stik } 24 \mathrm{fl} \mathrm{oz}(9 / 14)\end{array}$ & $6.5 \mathrm{~b}-\mathrm{g}$ & $32.8 \mathrm{a}-\mathrm{d}$ & $42.8 \mathrm{ab}$ & $936.1 \mathrm{a}-\mathrm{c}$ & $1940 \mathrm{c}-\mathrm{e}$ \\
\hline $\begin{array}{l}\text { 17. Admire Pro 4.6 SC } 8.5 \mathrm{fl} \mathrm{oz} \\
\text { + Proline 480 SC } 5.7 \mathrm{fl} \mathrm{oz} \mathrm{(F)} \\
\text { Propulse 400 SC } 13.6 \mathrm{fl} \mathrm{oz} \\
\text { Provost Silver } 3.52 \text { SC } 13 \mathrm{fl} \mathrm{oz} 7 / 17,8 / 13) \\
\text { Bravo Weather Stik } 24 \mathrm{fl} \mathrm{oz} \\
\text { + Tebuconazole } 3.6 \mathrm{~F} 7.2 \mathrm{fl} \mathrm{oz}(7 / 31,8 / 28) \\
\text { Bravo Weather Stik } 24 \mathrm{fl} \mathrm{oz}(9 / 14)\end{array}$ & $8.8 \mathrm{a}-\mathrm{e}$ & $36.0 \mathrm{ab}$ & $45.5 \mathrm{a}$ & $1028.4 \mathrm{ab}$ & $1191 \mathrm{ef}$ \\
\hline$P(\mathrm{~F})$ & 0.0008 & $<0.0001$ & $<0.0001$ & $<0.0001$ & $<0.0001$ \\
\hline LSD & 6.58 & 9.37 & 6.22 & 209.58 & 969.59 \\
\hline
\end{tabular}

z (F) In-furrow (16 May); (P) broadcast at pegging (1 Jul). First fungicide for leaf spot program was applied at R3 (17 Jul) and followed by applications on two-week timings as prescribed. All foliar treatments applied with Induce $0.125 \% \mathrm{v} / \mathrm{v}$.

y Counts of infection centers in the two center rows of each plot or a total of $70 \mathrm{ft}$ row. An infection center was a point with symptoms and/or signs of a disease and included 6 in. on either side of that point.

$x$ AUDPC $=$ area under the disease progress curve.

${ }^{w}$ Yields are weight of peanuts with moisture content adjusted to 7\%. Peanuts were dug 19 Oct and harvested 4 Nov. Means in a column followed by the same letter(s) are not significantly different according to Fisher's Protected LSD $(P=0.05)$. 
TEST ID: PTLFSPOT520

PURPOSE: To evaluate fungicide treatments for control of peanut leaf spot and Sclerotinia blight, and impact on yield at three locations, site 3

LOCATION: Tidewater AREC, 6321 Holland Rd., Suffolk, VA

CROP INFORMATION:

\begin{tabular}{|l|l|}
\hline Field & $46 \mathrm{~A}$ \\
\hline Crop history & 2019 corn, 2018 cotton, 2017 peanut \\
\hline Planting date & 15 May \\
\hline Variety & Sullivan \\
\hline Seeding rate & ca. 4 seed/row ft $(143 \mathrm{lb} / \mathrm{A})$ \\
\hline Plot length/width & $35^{\prime} \times 12^{\prime}$ \\
\hline Number of rows & 4 \\
\hline Row spacing & $36^{\prime \prime}$ \\
\hline Alleys (length between blocks) & $8^{\prime}$ \\
\hline Harvest date & 5 Nov \\
\hline
\end{tabular}

EXPERIMENTAL DESIGN: Randomized complete block with four replicates

TREATMENT APPLICATION:

\begin{tabular}{|l|l|l|}
\hline & IF liquid & Foliar spray \\
\hline Equipment & $\mathrm{CO}_{2}$ sprayer & Tractor plot sprayer \\
\hline Pressure $(\mathbf{p s i})$ & & $38 \mathrm{psi}$ \\
\hline Nozzle type & $.075 \mathrm{microtube}$ & $8002 \mathrm{TwinJ} \mathrm{et}$ \\
\hline Volume $(\mathbf{g a l} / \mathrm{A})$ & $5 \mathrm{gal} / \mathrm{A}$ & $19.88 \mathrm{gal} / \mathrm{A}$ \\
\hline Surfactant & & NIS $0.125 \% \mathrm{v} / \mathrm{v}$ \\
\hline
\end{tabular}

\section{APPLICATION SCHEDULE:}

\begin{tabular}{|l|l|}
\hline A & In-furrow (at planting) \\
\hline B & Pegging (49 DAP) \\
\hline C & R3 (63 DAP) \\
\hline D & R3 + 2 weeks (77 DAP) \\
\hline E & R3 + 4 weeks (91 DAP) \\
\hline F & R3 + 6 weeks (105 DAP) \\
\hline G & R3 + 8 weeks (119 DAP) \\
\hline
\end{tabular}

\section{TREATMENTS:}

\begin{tabular}{|l|l|l|l|l|}
\hline Trt \# & Product and formulation & Rate/A & Appl. timing & Appl. date \\
\hline 1 & Admire Pro 4.6 SC & $8.5 \mathrm{fl} \mathrm{oz}$ & & \\
& + Proline 480 SC & $5.7 \mathrm{fl} \mathrm{oz}$ & A & $15 \mathrm{May}$ \\
\hline 2 & Admire Pro 4.6 SC & $8.5 \mathrm{fl} \mathrm{oz}$ & & \\
& + Proline 480 SC & $5.7 \mathrm{fl} \mathrm{oz}$ & A & $15 \mathrm{May}$ \\
& Elatus 45 WG & $9.5 \mathrm{oz} \mathrm{wt}$ & & \\
& + Miravis 1.67 SC & $3.4 \mathrm{fl} \mathrm{oz}$ & $\mathrm{CE}$ & $17 \mathrm{Jul}, 12 \mathrm{Aug}$ \\
& Bravo Weather Stik & $24 \mathrm{fl} \mathrm{oz}$ & $\mathrm{G}$ & $14 \mathrm{Sep}$ \\
\hline 3 & Admire Pro 4.6 SC & $8.5 \mathrm{fl} \mathrm{oz}$ & & \\
& + Proline 480 SC & $5.7 \mathrm{fl} \mathrm{oz}$ & A & $15 \mathrm{May}$ \\
& Elatus 45 WG & $9.5 \mathrm{oz} \mathrm{wt}$ & & \\
& + Miravis 1.67 SC & $3.4 \mathrm{fl} \mathrm{oz}$ & DF & $30 \mathrm{Jul}, 27 \mathrm{Aug}$ \\
& Bravo Weather Stik & $24 \mathrm{fl} \mathrm{oz}$ & $\mathrm{G}$ & 14 Sep \\
\hline
\end{tabular}




\begin{tabular}{|c|c|c|c|c|}
\hline 4 & $\begin{array}{l}\text { Admire Pro 4.6SC } \\
\text { + Proline } 480 \mathrm{SC} \\
\text { Miravis 1.67 SC } \\
\text { + Tebuconazole } 3.6 \mathrm{~F} \\
\text { Bravo Weather Stik }\end{array}$ & $\begin{array}{l}8.5 \mathrm{fl} \mathrm{oz} \\
5.7 \mathrm{fl} \mathrm{oz} \\
3.4 \mathrm{fl} \mathrm{oz} \\
7.2 \mathrm{fl} \mathrm{oz} \\
24 \mathrm{fl} \mathrm{oz} \\
\end{array}$ & $\begin{array}{l}\mathrm{A} \\
\mathrm{CE} \\
\mathrm{G} \\
\end{array}$ & $\begin{array}{l}15 \text { May } \\
17 \text { Jul, } 12 \text { Aug } \\
14 \text { Sep }\end{array}$ \\
\hline 5 & $\begin{array}{l}\text { Admire Pro 4.6 SC } \\
\text { + Proline 480 SC } \\
\text { Miravis 1.67 SC } \\
\text { Elatus 45 WG } \\
\text { Bravo Weather Stik } \\
\end{array}$ & $\begin{array}{l}8.5 \mathrm{fl} \mathrm{oz} \\
5.7 \mathrm{fl} \mathrm{oz} \\
3.4 \mathrm{fl} \mathrm{oz} \\
9.5 \mathrm{oz} \mathrm{wt} \\
24 \mathrm{fl} \mathrm{oz} \\
\end{array}$ & $\begin{array}{l}\text { A } \\
\text { CE } \\
\text { DF } \\
\text { G }\end{array}$ & $\begin{array}{l}15 \text { May } \\
17 \text { Jul, } 12 \text { Aug } \\
30 \text { Jul, } 27 \text { Aug } \\
14 \text { Sep }\end{array}$ \\
\hline 6 & $\begin{array}{l}\text { Admire Pro 4.6 SC } \\
\text { + Proline 480 SC } \\
\text { Domark 230 ME } \\
\text { + Bravo Weather Stik } \\
\text { Bravo Weather Stik } \\
\text { + Tebuconazole 3.6 F } \\
\text { Bravo Weather Stik }\end{array}$ & $\begin{array}{l}8.5 \mathrm{fl} \mathrm{oz} \\
5.7 \mathrm{fl} \mathrm{oz} \\
2.5 \mathrm{fl} \mathrm{oz} \\
16 \mathrm{fl} \mathrm{oz} \\
24 \mathrm{fl} \mathrm{oz} \\
7.2 \mathrm{fl} \mathrm{oz} \\
24 \mathrm{fl} \mathrm{oz}\end{array}$ & $\begin{array}{l}\text { A } \\
\text { CE } \\
\text { DF } \\
\text { G }\end{array}$ & $\begin{array}{l}15 \text { May } \\
17 \text { Jul, } 12 \text { Aug } \\
\text { 30 Jul, } 27 \text { Aug } \\
14 \text { Sep }\end{array}$ \\
\hline 7 & $\begin{array}{l}\text { Admire Pro 4.6 SC } \\
+ \text { Proline } 480 \mathrm{SC} \\
\text { Miravis } 1.67 \mathrm{SC} \\
\text { Omega } 500 \mathrm{~F} \\
\text { Bravo Weather Stik }\end{array}$ & $\begin{array}{l}8.5 \mathrm{fl} \mathrm{oz} \\
5.7 \mathrm{fl} \mathrm{oz} \\
3.4 \mathrm{fl} \mathrm{oz} \\
24 \mathrm{fl} \mathrm{oz} \\
24 \mathrm{fl} \mathrm{oz}\end{array}$ & $\begin{array}{l}\text { A } \\
\text { CE } \\
\text { DF } \\
\text { G }\end{array}$ & $\begin{array}{l}15 \text { May } \\
17 \text { Jul, } 12 \text { Aug } \\
30 \text { Jul, } 27 \text { Aug } \\
14 \text { Sep }\end{array}$ \\
\hline 8 & $\begin{array}{l}\text { Admire Pro 4.6 SC } \\
\text { + Proline 480 SC } \\
\text { Elatus 45 WG } \\
\text { Bravo Weather Stik } \\
\text { + Tebuconazole 3.6 F } \\
\text { Bravo Weather Stik }\end{array}$ & $\begin{array}{l}8.5 \mathrm{fl} \mathrm{oz} \\
5.7 \mathrm{fl} \mathrm{oz} \\
9.5 \mathrm{oz} / \mathrm{wt} \\
24 \mathrm{fl} \mathrm{oz} \\
7.2 \mathrm{fl} \mathrm{oz} \\
24 \mathrm{fl} \mathrm{oz} \\
\end{array}$ & $\begin{array}{l}\mathrm{A} \\
\mathrm{CE} \\
\mathrm{DF} \\
\mathrm{G}\end{array}$ & $\begin{array}{l}15 \text { May } \\
17 \text { Jul, } 12 \text { Aug } \\
30 \text { Jul, } 27 \text { Aug } \\
14 \text { Sep }\end{array}$ \\
\hline 9 & $\begin{array}{l}\text { Admire Pro 4.6 SC } \\
+ \text { + Proline 480 SC } \\
\text { Priaxor 4.17 SC } \\
\text { Bravo Weather Stik } \\
\text { + Tebuconazole } 3.6 \text { F } \\
\text { Bravo Weather Stik }\end{array}$ & $\begin{array}{l}8.5 \mathrm{fl} \mathrm{oz} \\
5.7 \mathrm{fl} \mathrm{oz} \\
8 \mathrm{fl} \mathrm{oz} \\
24 \mathrm{fl} \mathrm{oz} \\
7.2 \mathrm{fl} \mathrm{oz} \\
24 \mathrm{fl} \mathrm{oz}\end{array}$ & $\begin{array}{l}\text { A } \\
\text { CE } \\
\text { DF } \\
\text { G }\end{array}$ & $\begin{array}{l}15 \text { May } \\
17 \text { Jul, } 12 \text { Aug } \\
30 \text { Jul, } 27 \text { Aug } \\
14 \text { Sep }\end{array}$ \\
\hline 10 & $\begin{array}{l}\text { Admire Pro 4.6 SC } \\
\text { + Proline 480 SC } \\
\text { Provost Silver 3.52 SC } \\
\text { Bravo Weather Stik } \\
\text { + Tebuconazole } 3.6 \text { F } \\
\text { Bravo Weather Stik }\end{array}$ & $\begin{array}{l}8.5 \mathrm{fl} \mathrm{oz} \\
5.7 \mathrm{fl} \mathrm{oz} \\
13 \mathrm{fl} \mathrm{oz} \\
24 \mathrm{fl} \mathrm{oz} \\
7.2 \mathrm{fl} \mathrm{oz} \\
24 \mathrm{fl} \mathrm{oz} \\
\end{array}$ & $\begin{array}{l}\text { A } \\
\text { CE } \\
\text { DF } \\
\text { G }\end{array}$ & $\begin{array}{l}15 \text { May } \\
17 \text { Jul, } 12 \text { Aug } \\
30 \text { Jul, } 27 \text { Aug } \\
14 \text { Sep }\end{array}$ \\
\hline 11 & $\begin{array}{l}\text { Admire Pro 4.6 SC } \\
+ \text { + Proline 480 SC } \\
\text { Lucento 4.17 SC } \\
\text { Bravo Weather Stik } \\
\text { + Tebuconazole } 3.6 \mathrm{~F} \\
\text { Bravo Weather Stik }\end{array}$ & $\begin{array}{l}8.5 \mathrm{fl} \mathrm{oz} \\
5.7 \mathrm{fl} \mathrm{oz} \\
5.5 \mathrm{fl} \mathrm{oz} \\
24 \mathrm{fl} \mathrm{oz} \\
7.2 \mathrm{fl} \mathrm{oz} \\
24 \mathrm{fl} \mathrm{oz} \\
\end{array}$ & $\begin{array}{l}\mathrm{A} \\
\mathrm{CE} \\
\mathrm{DF} \\
\mathrm{G}\end{array}$ & $\begin{array}{l}15 \text { May } \\
17 \text { Jul, } 12 \text { Aug } \\
30 \text { Jul, } 27 \text { Aug } \\
14 \text { Sep }\end{array}$ \\
\hline 12 & $\begin{array}{l}\text { Admire Pro 4.6 SC } \\
\text { + Proline 480 SC } \\
\text { Revytek SC } \\
\text { Bravo Weather Stik } \\
+ \text { Tebuconazole } 3.6 \mathrm{~F} \\
\text { Bravo Weather Stik }\end{array}$ & $\begin{array}{l}8.5 \mathrm{fl} \mathrm{oz} \\
5.7 \mathrm{fl} \mathrm{oz} \\
12 \mathrm{fl} \mathrm{oz} \\
24 \mathrm{fl} \mathrm{oz} \\
7.2 \mathrm{fl} \mathrm{oz} \\
24 \mathrm{fl} \mathrm{oz} \\
\end{array}$ & $\begin{array}{l}\mathrm{A} \\
\mathrm{CE} \\
\mathrm{DF} \\
\mathrm{G}\end{array}$ & $\begin{array}{l}15 \text { May } \\
17 \text { Jul, } 12 \text { Aug } \\
30 \text { Jul, } 27 \text { Aug } \\
14 \text { Sep }\end{array}$ \\
\hline
\end{tabular}




\begin{tabular}{|c|c|c|c|c|}
\hline 13 & $\begin{array}{l}\text { Admire Pro 4.6 SC } \\
\text { + Proline 480 SC } \\
\text { Excalia SC } \\
\text { Bravo Weather Stik } \\
\text { + Tebuconazole 3.6 F } \\
\text { Bravo Weather Stik } \\
\end{array}$ & $\begin{array}{l}8.5 \mathrm{fl} \mathrm{oz} \\
5.7 \mathrm{fl} \mathrm{oz} \\
2.65 \mathrm{fl} \mathrm{oz} \\
24 \mathrm{fl} \mathrm{oz} \\
7.2 \mathrm{fl} \mathrm{oz} \\
24 \mathrm{fl} \mathrm{oz} \\
\end{array}$ & $\begin{array}{l}\text { A } \\
\text { CE } \\
\text { DF } \\
\text { G }\end{array}$ & $\begin{array}{l}15 \text { May } \\
17 \text { Jul, } 12 \text { Aug } \\
\\
30 \text { Jul, } 27 \text { Aug } \\
14 \text { Sep }\end{array}$ \\
\hline 14 & $\begin{array}{l}\text { Admire Pro 4.6 SC } \\
\text { + Velum Prime } 500 \text { SC } \\
\text { Absolute 500 SC } \\
\text { Propulse 400 SC } \\
\text { + Elatus } 45 \text { WG } \\
\text { Bravo Weather Stik } \\
\text { Provost Silver 3.52 SC }\end{array}$ & $\begin{array}{l}8.5 \mathrm{fl} \mathrm{oz} \\
6.5 \mathrm{fl} \mathrm{oz} \\
3.5 \mathrm{fl} \mathrm{oz} \\
13.6 \mathrm{fl} \mathrm{oz} \\
7.3 \mathrm{wt} \mathrm{oz} \\
24 \mathrm{fl} \mathrm{oz} \\
13 \mathrm{fl} \mathrm{oz}\end{array}$ & $\begin{array}{l}\text { A } \\
\text { C } \\
\text { D } \\
\\
\text { EG } \\
\text { F }\end{array}$ & $\begin{array}{l}15 \text { May } \\
17 \text { Jul } \\
30 \text { Jul } \\
12 \text { Aug, } 14 \text { Sep } \\
27 \text { Aug }\end{array}$ \\
\hline 15 & $\begin{array}{l}\text { Admire Pro 4.6 SC } \\
+ \text { + Velum Prime } 500 \text { SC } \\
\text { Provost Silver } 3.52 \text { SC } \\
\text { Bravo Weather Stik } \\
+ \text { + Tebuconazole } 3.6 \text { F } \\
\text { Bravo Weather Stik }\end{array}$ & $\begin{array}{l}8.5 \mathrm{fl} \mathrm{oz} \\
15.84 \mathrm{fl} \mathrm{oz} \\
13 \mathrm{fl} \mathrm{oz} \\
24 \mathrm{fl} \mathrm{oz} \\
7.2 \mathrm{fl} \mathrm{oz} \\
24 \mathrm{fl} \mathrm{oz} \\
\end{array}$ & $\begin{array}{l}\text { A } \\
\text { CE } \\
\text { DF } \\
\text { G }\end{array}$ & $\begin{array}{l}15 \text { May } \\
17 \text { Jul, } 12 \text { Aug } \\
30 \text { Jul, } 27 \text { Aug } \\
14 \text { Sep }\end{array}$ \\
\hline 16 & $\begin{array}{l}\text { Admire Pro 4.6 SC } \\
\text { + Velum Prime 500 SC } \\
\text { Propulse 400 SC } \\
\text { Provost Silver 3.52 SC } \\
\text { Bravo Weather Stik } \\
\text { + Tebuconazole 3.6 F } \\
\text { Bravo Weather Stik }\end{array}$ & $\begin{array}{l}8.5 \mathrm{fl} \mathrm{oz} \\
15.84 \mathrm{fl} \mathrm{oz} \\
13.6 \mathrm{fl} \mathrm{oz} \\
13 \mathrm{fl} \mathrm{oz} \\
24 \mathrm{fl} \mathrm{oz} \\
7.2 \mathrm{fl} \mathrm{oz} \\
24 \mathrm{fl} \mathrm{oz} \\
\end{array}$ & $\begin{array}{l}\mathrm{A} \\
\mathrm{B} \\
\mathrm{CE} \\
\mathrm{DF} \\
\mathrm{G} \\
\end{array}$ & $\begin{array}{l}15 \text { May } \\
1 \text { Jul } \\
17 \text { Jul, } 12 \text { Aug } \\
30 \text { Jul, } 27 \text { Aug } \\
14 \text { Sep }\end{array}$ \\
\hline 17 & $\begin{array}{l}\text { Admire Pro 4.6 SC } \\
\text { + Proline 480 SC } \\
\text { Propulse 400 SC } \\
\text { Provost Silver } 3.52 \text { SC } \\
\text { Bravo Weather Stik } \\
\text { + Tebuconazole 3.6 F } \\
\text { Bravo Weather Stik }\end{array}$ & $\begin{array}{l}8.5 \mathrm{fl} \mathrm{oz} \\
5.7 \mathrm{fl} \mathrm{oz} \\
13.6 \mathrm{fl} \mathrm{oz} \\
13 \mathrm{fl} \mathrm{oz} \\
24 \mathrm{fl} \mathrm{oz} \\
7.2 \mathrm{fl} \mathrm{oz} \\
24 \mathrm{fl} \mathrm{oz}\end{array}$ & $\begin{array}{l}\text { A } \\
\text { B } \\
\text { CE } \\
\text { DF } \\
\text { G }\end{array}$ & $\begin{array}{l}\text { 15 May } \\
1 \text { Jul } \\
17 \text { Jul, } 12 \text { Aug } \\
\text { 30 Jul, } 27 \text { Aug } \\
\text { 14 Sep }\end{array}$ \\
\hline
\end{tabular}

SOIL TYPE: Nansemond fine sandy loam

SOIL FERTILITY REPORT (Jan 2020):

\begin{tabular}{|l|l|l|l|l|l|l|l|l|l|}
\hline $\mathbf{p H}$ & $\mathbf{P}(\mathbf{l b} / \mathbf{A})$ & $\mathbf{K}(\mathbf{l b} / \mathbf{A})$ & $\mathbf{C a}(\mathbf{l b} / \mathbf{A})$ & $\mathbf{M g}(\mathbf{l b} / \mathbf{A})$ & $\mathbf{Z n}(\mathbf{p p m})$ & $\mathbf{M n}(\mathbf{p p m})$ & $\mathbf{C u}(\mathbf{p p m})$ & $\mathbf{F e}(\mathbf{p p m})$ & $\mathbf{B}(\mathbf{p p m})$ \\
\hline 6.1 & 95 & 164 & 858 & 77 & 0.7 & 3.1 & 0.4 & 40.5 & 0.1 \\
\hline
\end{tabular}

MAINTENANCE CHEMICAL PROGRAMS:

\begin{tabular}{|l|l|}
\hline Fertilizer & Standard \\
\hline Herbicides & Standard \\
\hline Insecticides & Standard \\
\hline Fungicides & None except treatments \\
\hline Nematicides & None except treatments \\
\hline
\end{tabular}


MAINTENANCE CHEMICAL APPLICATIONS:

\begin{tabular}{|l|l|l|l|}
\hline Date & Type and target & Product and formulation & Rate/A \\
\hline $5 / 17 / 20$ & Herbicide & Strongarm & $0.45 \mathrm{fl} \mathrm{oz}$ \\
\hline $5 / 17 / 20$ & Herbicide & Dual II MAGNUM & $1.5 \mathrm{pt}$ \\
\hline $5 / 17 / 20$ & Herbicide & Prowl H2O & $1.0 \mathrm{pt}$ \\
\hline $5 / 17 / 20$ & Fertility & Boron & $1.0 \mathrm{qt}$ \\
\hline $6 / 4 / 20$ & Insecticide & Orthene $75 \mathrm{~S}$ & $12 \mathrm{oz}$ \\
\hline $6 / 25 / 20$ & Herbicide & Basagran & $1.5 \mathrm{pt}$ \\
\hline $6 / 25 / 20$ & Herbicide & Fusilade DX & $1 \mathrm{pt}$ \\
\hline $7 / 7 / 20$ & Herbicide & Storm & $1.5 \mathrm{pt}$ \\
\hline $7 / 7 / 20$ & Herbicide & Basagran & $1 \mathrm{pt}$ \\
\hline $7 / 13 / 20$ & Fertility & Manganese & $2 \mathrm{qt}$ \\
\hline $7 / 13 / 20$ & Insecticide & Danitol 2.4 EC & $8 \mathrm{fl} \mathrm{oz}$ \\
\hline $7 / 16 / 20$ & Fertility & Landplaster & $1500 \mathrm{lb}$ \\
\hline $7 / 24 / 20$ & Insecticide & Danitol 2.4 EC & $8 \mathrm{fl} \mathrm{oz}$ \\
\hline $8 / 6 / 20$ & Herbicide & Tide 2 EC $(\mathrm{Clethodim})$ & $16 \mathrm{fl} \mathrm{oz}$ \\
\hline
\end{tabular}


Table 1. Effect of fungicide treatment on disease incidence in peanut (PTLFSPOT520, Suffolk, VA 2020).

\begin{tabular}{|c|c|c|c|c|c|}
\hline \multirow{2}{*}{$\begin{array}{l}\text { Treatment, rate/A } \\
\text { and application timing/date }\end{array}$} & \multicolumn{4}{|c|}{ Leaf spot $(1-10)^{y}$} & \multirow{2}{*}{$\begin{array}{l}\text { \% leaf } \\
\text { spot }^{w} \\
9 \text { Oct }\end{array}$} \\
\hline & 25 Aug & 14 Sep & 30 Sep & AUDPC $^{x}$ & \\
\hline $\begin{array}{ll}\text { 1. } & \text { Admire Pro 4.6 SC } 8.5 \mathrm{fl} \mathrm{oz} \\
& + \text { Proline 480 SC } 5.7 \mathrm{fl} \mathrm{oz}(\mathrm{F})\end{array}$ & 1.3 & $2.5 \mathrm{a}$ & $5.5 \mathrm{a}$ & $101.5 \mathrm{a}$ & $75.0 \mathrm{a}$ \\
\hline $\begin{array}{ll}\text { 2. } & \text { Admire Pro 4.6 SC } 8.5 \mathrm{fl} \mathrm{oz} \\
& \text { + Proline 480 SC } 5.7 \mathrm{fl} \mathrm{oz}(\mathrm{F}) \\
& \text { Elatus 45 WG } 9.5 \mathrm{oz} / \mathrm{wt} \\
& + \text { Miravis } 1.67 \mathrm{SC} 3.4 \mathrm{fl} \mathrm{oz}(7 / 17,8 / 12) \\
& \text { Bravo Weather Stik } 24 \mathrm{fl} \mathrm{oz}(9 / 14)\end{array}$ & 1.0 & $1.0 \mathrm{c}$ & $1.3 \mathrm{bc}$ & $38.0 \mathrm{c}$ & $5.3 \mathrm{~b}$ \\
\hline $\begin{array}{ll}\text { 3. } & \text { Admire Pro 4.6 SC } 8.5 \mathrm{fl} \mathrm{oz} \\
\text { + Proline 480 SC } 5.7 \mathrm{fl} \mathrm{oz}(\mathrm{F}) \\
\text { Elatus 45 WG } 9.5 \mathrm{oz} / \mathrm{wt} \\
\text { + Miravis } 1.67 \text { SC } 3.4 \mathrm{fl} \mathrm{oz}(7 / 30,8 / 27) \\
\text { Bravo Weather Stik } 24 \mathrm{fl} \mathrm{oz}(9 / 14)\end{array}$ & 1.0 & $1.0 \mathrm{c}$ & $1.0 \mathrm{c}$ & $36.0 \mathrm{c}$ & $1.0 \mathrm{~b}$ \\
\hline $\begin{array}{l}\text { 4. } \\
\text { Admire Pro 4.6 SC } 8.5 \mathrm{fl} \mathrm{oz} \\
\text { + Proline 480 SC } 5.7 \mathrm{fl} \mathrm{oz}(\mathrm{F}) \\
\text { Miravis } 1.67 \text { SC } 3.4 \mathrm{fl} \mathrm{oz} \\
\text { + Tebuconazole } 3.6 \mathrm{~F} 7.2 \mathrm{fl} \mathrm{oz}(7 / 30,8 / 27) \\
\text { Bravo Weather Stik } 24 \mathrm{fl} \mathrm{oz}(9 / 14)\end{array}$ & 1.0 & $1.3 \mathrm{bc}$ & $1.5 \mathrm{~b}$ & $44.5 \mathrm{bc}$ & $3.0 \mathrm{~b}$ \\
\hline $\begin{array}{l}\text { 5. } \\
\text { + Proline } 480 \mathrm{SC} 5.7 \mathrm{fl} \mathrm{oz}(\mathrm{F}) \\
\text { Miravis } 1.67 \mathrm{SC} 3.4 \mathrm{fl} \mathrm{oz}(7 / 17,8 / 12) \\
\text { Elatus 45 WG 9.5 oz/wt }(7 / 30,8 / 27) \\
\text { Bravo Weather Stik } 24 \mathrm{fl} \mathrm{oz}(9 / 14)\end{array}$ & 1.0 & $1.0 \mathrm{c}$ & $1.0 \mathrm{c}$ & $36.0 \mathrm{c}$ & $1.0 \mathrm{~b}$ \\
\hline $\begin{array}{l}\text { 6. Admire Pro 4.6 SC } 8.5 \mathrm{fl} \mathrm{oz} \\
\text { + Proline } 480 \mathrm{SC} 5.7 \mathrm{fl} \mathrm{oz}(\mathrm{F}) \\
\text { Domark } 230 \mathrm{ME} 2.5 \mathrm{fl} \mathrm{oz} \\
\text { + Bravo Weather Stik } 16 \mathrm{fl} \mathrm{oz}(7 / 17,8 / 12) \\
\text { Bravo Weather Stik } 24 \mathrm{fl} \mathrm{oz} \\
\text { + Tebuconazole } 3.6 \mathrm{~F} 7.2 \mathrm{fl} \mathrm{oz}(7 / 30,8 / 27) \\
\text { Bravo Weather Stik } 24 \mathrm{fl} \mathrm{oz}(9 / 14)\end{array}$ & 1.0 & $1.0 \mathrm{c}$ & $1.0 \mathrm{c}$ & $36.0 \mathrm{c}$ & $1.0 \mathrm{~b}$ \\
\hline $\begin{array}{l}\text { 7. } \\
\text { Admire Pro 4.6 SC } 8.5 \mathrm{fl} \mathrm{oz} \\
\text { + Proline 480 SC } 5.7 \mathrm{fl} \mathrm{oz} \mathrm{(F)} \\
\text { Miravis 1.67 SC } 3.4 \mathrm{fl} \mathrm{oz}(7 / 17,8 / 12) \\
\text { Omega 500 F 24 fl oz (7/30, 8/27) } \\
\text { Bravo Weather Stik } 24 \mathrm{fl} \mathrm{oz}(9 / 14) \\
\end{array}$ & 1.0 & $1.0 \mathrm{c}$ & $1.3 \mathrm{bc}$ & $38.0 \mathrm{c}$ & $3.3 \mathrm{~b}$ \\
\hline $\begin{array}{ll}\text { 8. } & \text { Admire Pro 4.6 SC } 8.5 \mathrm{fl} \mathrm{oz} \\
& + \text { Proline } 480 \mathrm{SC} 5.7 \mathrm{fl} \mathrm{oz}(\mathrm{F}) \\
\text { Elatus } 45 \mathrm{WG} 9.5 \mathrm{oz} / \mathrm{wt}(7 / 17,8 / 12) \\
\text { Bravo Weather Stik } 24 \mathrm{fl} \mathrm{oz} \\
\text { + Tebuconazole } 3.6 \mathrm{~F} 7.2 \mathrm{fl} \mathrm{oz}(7 / 30,8 / 27) \\
\text { Bravo Weather Stik } 24 \mathrm{fl} \mathrm{oz}(9 / 14)\end{array}$ & 1.0 & $1.0 \mathrm{c}$ & $1.0 \mathrm{c}$ & $36.0 \mathrm{c}$ & $1.0 \mathrm{~b}$ \\
\hline $\begin{array}{l}\text { 9. Admire Pro 4.6 SC } 8.5 \mathrm{fl} \mathrm{oz} \\
\text { + Proline 480 SC } 5.7 \mathrm{fl} \mathrm{oz}(\mathrm{F}) \\
\text { Priaxor 4.17 SC } 8 \mathrm{fl} \mathrm{oz}(7 / 17,8 / 12) \\
\text { Bravo Weather Stik } 24 \mathrm{fl} \mathrm{oz} \\
\text { + Tebuconazole } 3.6 \mathrm{~F} 7.2 \mathrm{fl} \mathrm{oz}(7 / 30,8 / 27) \\
\text { Bravo Weather Stik } 24 \mathrm{fl} \mathrm{oz}(9 / 14)\end{array}$ & 1.0 & $1.3 \mathrm{bc}$ & $1.3 \mathrm{bc}$ & $42.5 \mathrm{bc}$ & $2.0 \mathrm{~b}$ \\
\hline $\begin{array}{l}\text { 10. } \\
\text { Admire Pro 4.6 SC } 8.5 \mathrm{fl} \mathrm{oz} \\
\text { + Proline 480 SC 5.7 fl oz (F) } \\
\text { Provost Silver 3.52 SC } 13 \mathrm{fl} \mathrm{oz}(7 / 17,8 / 12) \\
\text { Bravo Weather Stik } 24 \mathrm{fl} \mathrm{oz} \\
\text { + Tebuconazole } 3.6 \mathrm{~F} 7.2 \mathrm{fl} \mathrm{oz}(7 / 30,8 / 27) \\
\text { Bravo Weather Stik } 24 \mathrm{fl} \mathrm{oz}(9 / 14)\end{array}$ & 1.0 & $1.3 \mathrm{bc}$ & $1.0 \mathrm{c}$ & $40.5 \mathrm{bc}$ & $2.0 \mathrm{~b}$ \\
\hline $\begin{array}{l}\text { 11. Admire Pro 4.6 SC } 8.5 \mathrm{fl} \mathrm{oz} \\
\text { + Proline 480 SC } 5.7 \mathrm{fl} \mathrm{oz} \mathrm{(F)} \\
\text { Lucento 4.17 SC } 5.5 \mathrm{fl} \mathrm{oz}(7 / 13,8 / 12) \\
\text { Bravo Weather Stik } 24 \mathrm{fl} \mathrm{oz} \\
\text { + Tebuconazole 3.6 F } 7.2 \mathrm{fl} \mathrm{oz}(7 / 30,8 / 27) \\
\text { Bravo Weather Stik } 24 \mathrm{fl} \mathrm{oz}(9 / 14)\end{array}$ & 1.0 & $1.8 \mathrm{~b}$ & $1.0 \mathrm{c}$ & $49.5 \mathrm{~b}$ & $2.0 \mathrm{~b}$ \\
\hline
\end{tabular}




\begin{tabular}{|c|c|c|c|c|c|}
\hline $\begin{array}{l}\text { 12. } \\
\text { Admire Pro 4.6 SC } 8.5 \mathrm{fl} \mathrm{oz} \\
+ \text { Proline } 480 \text { SC } 5.7 \mathrm{fl} \mathrm{oz} \mathrm{(F)} \\
\text { Revytek SC } 12 \mathrm{fl} \mathrm{oz}(7 / 17,8 / 12) \\
\text { Bravo Weather Stik } 24 \mathrm{fl} \mathrm{oz} \\
+ \text { Tebuconazole } 3.6 \mathrm{~F} 7.2 \mathrm{fl} \mathrm{oz}(7 / 30,8 / 27) \\
\text { Bravo Weather Stik } 24 \mathrm{fl} \mathrm{oz}(9 / 14)\end{array}$ & 1.0 & $1.0 \mathrm{c}$ & $1.0 \mathrm{c}$ & $36.0 \mathrm{c}$ & $1.0 \mathrm{~b}$ \\
\hline $\begin{array}{l}\text { 13. Admire Pro 4.6 SC } 8.5 \mathrm{fl} \mathrm{oz} \\
+ \text { Proline } 480 \text { SC } 5.7 \mathrm{fl} \mathrm{oz}(\mathrm{F}) \\
\text { Excalia SC } 2.65 \mathrm{fl} \mathrm{oz}(7 / 17,8 / 12) \\
\text { Bravo Weather Stik } 24 \mathrm{fl} \mathrm{oz} \\
\text { + Tebuconazole } 3.6 \mathrm{~F} 7.2 \mathrm{fl} \mathrm{oz}(7 / 30,8 / 27) \\
\text { Bravo Weather Stik } 24 \mathrm{fl} \mathrm{oz}(9 / 14)\end{array}$ & 1.0 & $1.3 \mathrm{bc}$ & $1.0 \mathrm{c}$ & $40.5 \mathrm{bc}$ & $2.0 \mathrm{~b}$ \\
\hline $\begin{array}{l}\text { 14. Admire Pro 4.6 SC } 8.5 \mathrm{fl} \mathrm{oz} \\
\text { + Velum Prime } 500 \mathrm{SC} 15.84 \mathrm{fl} \mathrm{oz}(\mathrm{F}) \\
\text { Absolute 500 SC } 3.5 \mathrm{fl} \mathrm{oz}(7 / 17) \\
\text { Propulse 400 SC } 13.6 \mathrm{fl} \mathrm{oz} \\
\text { + Elatus 45 WG } 7.3 \mathrm{wt} / \mathrm{oz}(7 / 30) \\
\text { Bravo Weather Stik } 24 \mathrm{fl} \mathrm{oz}(8 / 12,9 / 14) \\
\text { Provost Silver } 3.52 \mathrm{SC} 13 \mathrm{fl} \mathrm{oz}(8 / 27) \\
\end{array}$ & 1.0 & $1.0 \mathrm{c}$ & $1.5 \mathrm{~b}$ & $40.0 \mathrm{bc}$ & $3.3 \mathrm{~b}$ \\
\hline $\begin{array}{l}\text { 15. Admire Pro 4.6 SC } 8.5 \mathrm{fl} \mathrm{oz} \\
+ \text { Velum Prime 500 SC } 6.5 \mathrm{fl} \mathrm{oz}(\mathrm{F}) \\
\text { Provost Silver 3.52 SC } 13 \mathrm{fl} \mathrm{oz}(7 / 17,8 / 12) \\
\text { Bravo Weather Stik } 24 \mathrm{fl} \mathrm{oz} \\
+ \text { Tebuconazole 3.6 F } 7.2 \mathrm{fl} \mathrm{oz}(7 / 30,8 / 27) \\
\text { Bravo Weather Stik } 24 \mathrm{fl} \mathrm{oz}(9 / 14)\end{array}$ & 1.0 & $1.0 \mathrm{c}$ & $1.0 \mathrm{c}$ & $36.0 \mathrm{c}$ & $1.0 \mathrm{~b}$ \\
\hline $\begin{array}{l}\text { 16. Admire Pro 4.6 SC } 8.5 \mathrm{fl} \mathrm{oz} \\
+ \text { Velum Prime } 500 \mathrm{SC} 15.84 \mathrm{fl} \mathrm{oz}(\mathrm{F}) \\
\text { Propulse 400 SC } 13.6 \mathrm{fl} \mathrm{oz} \\
\text { Provost Silver 3.52 SC } 13 \mathrm{fl} \mathrm{oz}(7 / 13,8 / 12) \\
\text { Bravo Weather Stik } 24 \mathrm{fl} \mathrm{oz} \\
+ \text { Tebuconazole } 3.6 \mathrm{~F} 7.2 \mathrm{fl} \mathrm{oz}(7 / 30,8 / 27) \\
\text { Bravo Weather Stik } 24 \mathrm{fl} \mathrm{oz}(9 / 14) \\
\end{array}$ & 1.0 & $1.0 \mathrm{c}$ & $1.0 \mathrm{c}$ & $36.0 \mathrm{c}$ & $2.0 \mathrm{~b}$ \\
\hline $\begin{array}{l}\text { 17. Admire Pro 4.6 SC } 8.5 \mathrm{fl} \mathrm{oz} \\
\text { + Proline 480 SC } 5.7 \mathrm{fl} \mathrm{oz} \mathrm{(F)} \\
\text { Propulse 400 SC } 13.6 \mathrm{fl} \mathrm{oz} \\
\text { Provost Silver } 3.52 \text { SC } 13 \mathrm{fl} \mathrm{oz} 7 / 17,8 / 12) \\
\text { Bravo Weather Stik } 24 \mathrm{fl} \mathrm{oz} \\
\text { + Tebuconazole } 3.6 \mathrm{~F} 7.2 \mathrm{fl} \mathrm{oz}(7 / 30,8 / 27) \\
\text { Bravo Weather Stik } 24 \mathrm{fl} \mathrm{oz}(9 / 14)\end{array}$ & 1.0 & $1.0 \mathrm{c}$ & $1.0 \mathrm{c}$ & $36.0 \mathrm{c}$ & $1.0 \mathrm{~b}$ \\
\hline$P(\mathrm{~F})$ & 0.47 & $<0.0001$ & $<0.0001$ & $<0.0001$ & $<0.0001$ \\
\hline LSD & N.S. & 0.53 & 0.45 & 11.00 & 7.21 \\
\hline
\end{tabular}

z (F) In-furrow (15 May); (P) broadcast at pegging (1 Jul). First fungicide for leaf spot program was applied at R3 (17 Jul) and followed by applications on two-week timings as prescribed. All foliar treatments applied with Induce $0.125 \% \mathrm{v} / \mathrm{v}$.

y Florida leaf spot scale: $1=$ no disease, $10=$ dead plants, Chiteka et al. (1988). Untreated control (Trt. 1) plots were observed for leaf spot on $7 \mathrm{Aug}$; little to no lesions observed.

$x$ AUDPC $=$ area under the disease progress curve.

${ }^{w}$ Percent of leaves in two treatment rows with symptoms of leaf spot. Means in a column followed by the same letter(s) are not significantly different according to Fisher's Protected LSD $(P=0.05)$. Arcsine transformation of percentage data was made in analysis to determine statistical significance. 
Table 2. Effect of fungicide treatment on soilborne disease and yield in peanut (PTLFSPOT520, Suffolk, VA 2020).

\begin{tabular}{|c|c|c|c|c|c|c|}
\hline \multirow{2}{*}{$\begin{array}{l}\text { Treatment, rate/A } \\
\text { and application timing/date }{ }^{\mathrm{z}}\end{array}$} & \multicolumn{5}{|c|}{ Sclerotinia blight $^{\mathbf{y}}$} & \multirow{2}{*}{$\begin{array}{c}\text { Yield } \\
(\mathbf{l b} / \mathbf{A})^{\mathrm{w}}\end{array}$} \\
\hline & 28 Aug & 14 Sep & 30 Sep & 20 Oct & AUDPC $^{x}$ & \\
\hline $\begin{array}{l}\text { 1. Admire Pro 4.6 SC } 8.5 \mathrm{fl} \mathrm{oz} \\
\text { + Proline 480 SC } 5.7 \mathrm{fl} \mathrm{oz}(\mathrm{F})\end{array}$ & 2.0 & $5.8 \mathrm{a}-\mathrm{d}$ & $15.8 \mathrm{a}-\mathrm{c}$ & $25.0 \mathrm{a}-\mathrm{d}$ & $645.4 \mathrm{a}-\mathrm{d}$ & 3008 \\
\hline $\begin{array}{ll}\text { 2. } & \text { Admire Pro 4.6 SC } 8.5 \mathrm{fl} \mathrm{oz} \\
& + \text { Proline } 480 \mathrm{SC} 5.7 \mathrm{fl} \mathrm{oz}(\mathrm{F}) \\
& \text { Elatus 45 WG } 9.5 \mathrm{oz} / \mathrm{wt} \\
& + \text { Miravis } 1.67 \text { SC } 3.4 \mathrm{fl} \mathrm{oz}(7 / 17,8 / 12) \\
& \text { Bravo Weather Stik } 24 \mathrm{fl} \mathrm{oz}(9 / 14) \\
\end{array}$ & 0.0 & $0.3 \mathrm{~g}$ & $3.8 \mathrm{de}$ & $12.5 \mathrm{ef}$ & $196.6 \mathrm{ef}$ & 4376 \\
\hline $\begin{array}{ll}\text { 3. } & \text { Admire Pro 4.6 SC } 8.5 \mathrm{fl} \mathrm{oz} \\
\text { + Proline } 480 \text { SC } 5.7 \mathrm{fl} \mathrm{oz}(\mathrm{F}) \\
\text { Elatus } 45 \mathrm{WG} 9.5 \mathrm{oz} / \mathrm{wt} \\
\text { + Miravis } 1.67 \mathrm{SC} 3.4 \mathrm{fl} \mathrm{oz}(7 / 30,8 / 27) \\
\text { Bravo Weather Stik } 24 \mathrm{fl} \mathrm{oz}(9 / 14) \\
\end{array}$ & 1.0 & $3.0 \mathrm{c}-\mathrm{g}$ & $4.8 \mathrm{c}-\mathrm{e}$ & $13.0 \mathrm{~d}-\mathrm{f}$ & $273.5 \mathrm{~d}-\mathrm{f}$ & 4012 \\
\hline $\begin{array}{ll}\text { 4. } & \text { Admire Pro 4.6 SC } 8.5 \mathrm{fl} \mathrm{oz} \\
\text { + Proline } 480 \text { SC } 5.7 \mathrm{fl} \mathrm{oz} \mathrm{(F)} \\
\text { Miravis } 1.67 \text { SC } 3.4 \mathrm{fl} \mathrm{oz} \\
\text { + Tebuconazole } 3.6 \mathrm{~F} 7.2 \mathrm{fl} \mathrm{oz}(7 / 30,8 / 27) \\
\text { Bravo Weather Stik } 24 \mathrm{fl} \mathrm{oz}(9 / 14) \\
\end{array}$ & 0.0 & $1.0 \mathrm{e}-\mathrm{g}$ & $5.8 \mathrm{c}-\mathrm{e}$ & $15.5 \mathrm{c}-\mathrm{f}$ & $275.0 \mathrm{~d}-\mathrm{f}$ & 3861 \\
\hline $\begin{array}{ll}\text { 5. } & \text { Admire Pro 4.6 SC } 8.5 \mathrm{fl} \mathrm{oz} \\
\text { + Proline 480 SC } 5.7 \mathrm{fl} \mathrm{oz}(\mathrm{F}) \\
\text { Miravis } 1.67 \mathrm{SC} 3.4 \mathrm{fl} \mathrm{oz}(7 / 17,8 / 12) \\
\text { Elatus 45 WG } 9.5 \mathrm{oz} / \mathrm{wt}(7 / 30,8 / 27) \\
\text { Bravo Weather Stik } 24 \mathrm{fl} \mathrm{oz}(9 / 14) \\
\end{array}$ & 0.0 & $0.5 \mathrm{~g}$ & $9.5 \mathrm{~b}-\mathrm{e}$ & $11.0 \mathrm{f}$ & $289.3 \mathrm{~d}-\mathrm{f}$ & 3785 \\
\hline $\begin{array}{ll}\text { 6. } & \text { Admire Pro 4.6 SC } 8.5 \mathrm{fl} \mathrm{oz} \\
\text { + Proline 480 SC } 5.7 \mathrm{fl} \mathrm{oz} \mathrm{(F)} \\
\text { Domark 230 ME } 2.5 \mathrm{fl} \mathrm{oz} \\
\text { + Bravo Weather Stik } 16 \mathrm{fl} \mathrm{oz}(7 / 17,8 / 12) \\
\text { Bravo Weather Stik } 24 \mathrm{fl} \mathrm{oz} \\
\text { + Tebuconazole 3.6 F } 7.2 \mathrm{fl} \mathrm{oz}(7 / 30,8 / 27) \\
\text { Bravo Weather Stik } 24 \mathrm{fl} \mathrm{oz}(9 / 14) \\
\end{array}$ & 4.8 & $8.0 \mathrm{ab}$ & $19.8 \mathrm{ab}$ & $29.8 \mathrm{ab}$ & $825.4 \mathrm{ab}$ & 2692 \\
\hline $\begin{array}{ll}7 . & \text { Admire Pro 4.6 SC } 8.5 \mathrm{fl} \mathrm{oz} \\
\text { + Proline } 480 \text { SC } 5.7 \mathrm{fl} \mathrm{oz}(\mathrm{F}) \\
\text { Miravis } 1.67 \mathrm{SC} 3.4 \mathrm{fl} \mathrm{oz}(7 / 17,8 / 12) \\
\text { Omega 500 F } 24 \mathrm{fl} \mathrm{oz}(7 / 30,8 / 27) \\
\text { Bravo Weather Stik } 24 \mathrm{fl} \mathrm{oz}(9 / 14) \\
\end{array}$ & 0.0 & $0.8 \mathrm{fg}$ & $0.8 \mathrm{e}$ & $11.3 \mathrm{f}$ & $138.4 \mathrm{f}$ & 3849 \\
\hline $\begin{array}{ll}\text { 8. } & \text { Admire Pro 4.6 SC } 8.5 \mathrm{fl} \mathrm{oz} \\
& + \text { Proline } 480 \text { SC } 5.7 \mathrm{fl} \mathrm{oz}(\mathrm{F}) \\
\text { Elatus 45 WG } 9.5 \mathrm{oz} / \mathrm{wt}(7 / 17,8 / 12) \\
\text { Bravo Weather Stik } 24 \mathrm{fl} \mathrm{oz} \\
\text { + Tebuconazole } 3.6 \mathrm{~F} 7.2 \mathrm{fl} \mathrm{oz}(7 / 30,8 / 27) \\
\text { Bravo Weather Stik } 24 \mathrm{fl} \mathrm{oz}(9 / 14)\end{array}$ & 1.0 & $3.5 \mathrm{~b}-\mathrm{g}$ & $12.8 \mathrm{a}-\mathrm{d}$ & $24.3 \mathrm{a}-\mathrm{e}$ & $538.3 \mathrm{a}-\mathrm{e}$ & 3416 \\
\hline $\begin{array}{l}\text { 9. } \\
\text { Admire Pro 4.6 SC } 8.5 \mathrm{fl} \mathrm{oz} \\
\text { + Proline 480 SC } 5.7 \mathrm{fl} \mathrm{oz} \mathrm{(F)} \\
\text { Priaxor 4.17 SC } 8 \mathrm{fl} \mathrm{oz} \mathrm{(7/17,} \mathrm{8/12)} \\
\text { Bravo Weather Stik } 24 \mathrm{fl} \mathrm{oz} \\
\text { + Tebuconazole 3.6 F } 7.2 \mathrm{fl} \mathrm{oz}(7 / 30,8 / 27) \\
\text { Bravo Weather Stik } 24 \mathrm{fl} \mathrm{oz}(9 / 14) \\
\end{array}$ & 0.3 & $2.5 \mathrm{c}-\mathrm{g}$ & $8.5 \mathrm{c}-\mathrm{e}$ & $19.3 \mathrm{~b}-\mathrm{f}$ & $388.9 \mathrm{c}-\mathrm{f}$ & 3435 \\
\hline $\begin{array}{l}\text { 10. } \\
\text { + Prolinire Pro 4.6 SC } 8.5 \mathrm{fl} \mathrm{oz} \\
\text { Provost Silver 3.52 SC } 13 \mathrm{fl} \mathrm{oz}(7 / 17,8 / 12) \\
\text { Bravo Weather Stik } 24 \mathrm{fl} \mathrm{oz} \\
\text { + Tebuconazole 3.6 F } 7.2 \mathrm{fl} \mathrm{oz}(7 / 30,8 / 27) \\
\text { Bravo Weather Stik } 24 \mathrm{fl} \mathrm{oz}(9 / 14) \\
\end{array}$ & 2.8 & $5.3 \mathrm{a}-\mathrm{f}$ & $11.8 \mathrm{a}-\mathrm{e}$ & $19.5 \mathrm{~b}-\mathrm{f}$ & $516.5 \mathrm{~b}-\mathrm{f}$ & 3321 \\
\hline $\begin{array}{l}\text { 11. Admire Pro 4.6 SC } 8.5 \mathrm{fl} \mathrm{oz} \\
\text { + Proline 480 SC } 5.7 \mathrm{fl} \mathrm{oz} \mathrm{(F)} \\
\text { Lucento 4.17 SC } 5.5 \mathrm{fl} \mathrm{oz}(7 / 13,8 / 12) \\
\text { Bravo Weather Stik } 24 \mathrm{fl} \mathrm{oz} \\
\text { + Tebuconazole 3.6 F } 7.2 \mathrm{fl} \mathrm{oz}(7 / 30,8 / 27) \\
\text { Bravo Weather Stik } 24 \mathrm{fl} \mathrm{oz}(9 / 14)\end{array}$ & 0.8 & $4.3 \mathrm{~b}-\mathrm{g}$ & $10.3 \mathrm{~b}-\mathrm{e}$ & $21.0 \mathrm{a}-\mathrm{f}$ & $471.0 \mathrm{~b}-\mathrm{f}$ & 3370 \\
\hline
\end{tabular}




\begin{tabular}{|c|c|c|c|c|c|c|}
\hline $\begin{array}{l}\text { 12. } \\
\text { Admire Pro 4.6 SC } 8.5 \mathrm{fl} \mathrm{oz} \\
+ \text { Proline 480 SC } 5.7 \mathrm{fl} \mathrm{oz} \mathrm{(F)} \\
\text { Revytek SC } 12 \mathrm{fl} \mathrm{oz}(7 / 17,8 / 12) \\
\text { Bravo Weather Stik } 24 \mathrm{fl} \mathrm{oz} \\
\text { + Tebuconazole 3.6 F } 7.2 \mathrm{fl} \mathrm{oz} \mathrm{(7/30,} \mathrm{8/27)} \\
\text { Bravo Weather Stik } 24 \mathrm{fl} \mathrm{oz}(9 / 14) \\
\end{array}$ & 3.3 & $5.5 \mathrm{a}-\mathrm{e}$ & $15.3 \mathrm{a}-\mathrm{c}$ & $28.8 \mathrm{ab}$ & $680.4 \mathrm{a}-\mathrm{c}$ & 2662 \\
\hline $\begin{array}{l}\text { 13. Admire Pro 4.6 SC } 8.5 \mathrm{fl} \mathrm{oz} \\
+ \text { Proline } 480 \text { SC } 5.7 \mathrm{fl} \mathrm{oz}(\mathrm{F}) \\
\text { Excalia SC } 2.65 \mathrm{fl} \mathrm{oz}(7 / 17,8 / 12) \\
\text { Bravo Weather Stik } 24 \mathrm{fl} \mathrm{oz} \\
\text { + Tebuconazole } 3.6 \mathrm{~F} 7.2 \mathrm{fl} \mathrm{oz}(7 / 30,8 / 27) \\
\text { Bravo Weather Stik } 24 \mathrm{fl} \mathrm{oz}(9 / 14)\end{array}$ & 2.8 & $9.5 \mathrm{a}$ & $22.0 \mathrm{a}$ & $33.0 \mathrm{a}$ & $906.1 \mathrm{a}$ & 2434 \\
\hline $\begin{array}{l}\text { 14. Admire Pro 4.6 SC } 8.5 \mathrm{fl} \mathrm{oz} \\
+ \text { Velum Prime } 500 \mathrm{SC} 15.84 \mathrm{fl} \mathrm{oz}(\mathrm{F}) \\
\text { Absolute 500 SC } 3.5 \mathrm{fl} \mathrm{oz}(7 / 17) \\
\text { Propulse 400 SC } 13.6 \mathrm{fl} \mathrm{oz} \\
+ \text { Elatus } 45 \mathrm{WG} 7.3 \mathrm{wt} / \mathrm{oz}(7 / 30) \\
\text { Bravo Weather Stik } 24 \mathrm{fl} \mathrm{oz}(8 / 12,9 / 14) \\
\text { Provost Silver } 3.52 \text { SC } 13 \mathrm{fl} \mathrm{oz}(8 / 27) \\
\end{array}$ & 0.5 & $2.3 \mathrm{~d}-\mathrm{g}$ & $9.0 \mathrm{~b}-\mathrm{e}$ & $18.0 \mathrm{~b}-\mathrm{f}$ & $383.4 \mathrm{c}-\mathrm{f}$ & 3569 \\
\hline $\begin{array}{l}\text { 15. Admire Pro 4.6 SC } 8.5 \mathrm{fl} \mathrm{oz} \\
+ \text { Velum Prime 500 SC } 6.5 \mathrm{fl} \mathrm{oz}(\mathrm{F}) \\
\text { Provost Silver 3.52 SC } 13 \mathrm{fl} \mathrm{oz}(7 / 17,8 / 12) \\
\text { Bravo Weather Stik } 24 \mathrm{fl} \mathrm{oz} \\
+ \text { Tebuconazole 3.6 F } 7.2 \mathrm{fl} \mathrm{oz}(7 / 30,8 / 27) \\
\text { Bravo Weather Stik } 24 \mathrm{fl} \mathrm{oz}(9 / 14)\end{array}$ & 1.0 & $3.5 \mathrm{~b}-\mathrm{g}$ & $11.5 \mathrm{a}-\mathrm{e}$ & $20.3 b-f$ & $475.8 \mathrm{~b}-\mathrm{f}$ & 3309 \\
\hline $\begin{array}{l}\text { 16. Admire Pro 4.6 SC } 8.5 \mathrm{fl} \mathrm{oz} \\
+ \text { Velum Prime 500 SC } 15.84 \mathrm{fl} \mathrm{oz}(\mathrm{F}) \\
\text { Propulse 400 SC } 13.6 \mathrm{fl} \mathrm{oz} \\
\text { Provost Silver 3.52 SC } 13 \mathrm{fl} \mathrm{oz}(7 / 13,8 / 12) \\
\text { Bravo Weather Stik } 24 \mathrm{fl} \mathrm{oz} \\
+ \text { Tebuconazole } 3.6 \mathrm{~F} 7.2 \mathrm{fl} \mathrm{oz}(7 / 30,8 / 27) \\
\text { Bravo Weather Stik } 24 \mathrm{fl} \mathrm{oz}(9 / 14)\end{array}$ & 0.8 & $3.0 \mathrm{c}-\mathrm{g}$ & $10.8 \mathrm{~b}-\mathrm{e}$ & $20.0 \mathrm{~b}-\mathrm{f}$ & $449.4 \mathrm{~b}-\mathrm{f}$ & 3027 \\
\hline $\begin{array}{l}\text { 17. Admire Pro 4.6 SC } 8.5 \mathrm{fl} \mathrm{oz} \\
\text { + Proline 480 SC } 5.7 \mathrm{fl} \mathrm{oz} \mathrm{(F)} \\
\text { Propulse 400 SC } 13.6 \mathrm{fl} \mathrm{oz} \\
\text { Provost Silver 3.52 SC } 13 \mathrm{fl} \mathrm{oz} 7 / 17,8 / 12) \\
\text { Bravo Weather Stik } 24 \mathrm{fl} \mathrm{oz} \\
\text { + Tebuconazole 3.6 F } 7.2 \mathrm{fl} \mathrm{oz}(7 / 30,8 / 27) \\
\text { Bravo Weather Stik } 24 \mathrm{fl} \mathrm{oz}(9 / 14) \\
\end{array}$ & 2.5 & $7.0 \mathrm{a}-\mathrm{c}$ & $12.8 \mathrm{a}-\mathrm{d}$ & $26.8 \mathrm{a}-\mathrm{c}$ & $633.8 \mathrm{a}-\mathrm{d}$ & 2908 \\
\hline$P(\mathrm{~F})$ & 0.10 & 0.005 & 0.03 & 0.01 & 0.007 & 0.10 \\
\hline LSD & N.S. & 4.70 & 11.03 & 12.39 & 384.03 & N.S. \\
\hline
\end{tabular}

z (F) In-furrow (15 May); (P) broadcast at pegging (1 Jul). First fungicide for leaf spot program was applied at R3 (17 Jul) and followed by applications on two-week timings as prescribed. All foliar treatments applied with Induce $0.125 \% \mathrm{v} / \mathrm{v}$.

y Counts of infection centers in the two center rows of each plot or a total of $70 \mathrm{ft}$ row. An infection center was a point with symptoms and/or signs of a disease and included 6 in. on either side of that point.

$x$ AUDPC $=$ area under the disease progress curve.

${ }^{w}$ Yields are weight of peanuts with moisture content adjusted to 7\%. Peanuts were dug 20 Oct and harvested 5 Nov. Means in a column followed by the same letter(s) are not significantly different according to Fisher's Protected LSD $(P=0.05)$. 
TEST ID: COTSEEDFUN120

PURPOSE: To evaluate fungicide seed treatments and seeding rate for control of damping-off diseases and impact on plant health and yield in cotton

LOCATION: Tidewater Research Farm, Hare Rd., Suffolk, VA

CROP INFORMATION:

\begin{tabular}{|l|l|}
\hline Field & $16 \mathrm{~B}$ \\
\hline Crop history & 2019 peanut, 2018 corn, 2017 cotton \\
\hline Planting date & 15 May \\
\hline Variety & DP 1646 B2XF \\
\hline Seeding rate & $3.5-4$ seed/row ft \\
\hline Plot length/width & $30^{\prime}$ \\
\hline Number of rows & 2 \\
\hline Row spacing & $36^{\prime \prime}$ \\
\hline Alleys (length between blocks) & $8^{\prime}$ \\
\hline Harvest date & 3 Dec \\
\hline
\end{tabular}

EXPERIMENTAL DESIGN: Randomized complete block with four replicates

TREATMENTS:

\begin{tabular}{|c|c|c|c|}
\hline Trt. & \# Seed/ft & Seed treatment & In-furrow trt and rate/A \\
\hline 1 & 3 & $\begin{array}{l}\text { Spera } 1.8 \mathrm{oz}+\text { Vortex } 0.08 \mathrm{oz}+\text { Allegiance FL } 0.75 \mathrm{oz} \\
+ \text { EverGol Prime } 0.32 \mathrm{oz} / \mathrm{cwt}\end{array}$ & -- \\
\hline 2 & 4 & $\begin{array}{l}\text { Spera } 1.8 \mathrm{oz}+\text { Vortex } 0.08 \mathrm{oz}+\text { Allegiance FL } 0.75 \mathrm{oz} \\
+ \text { EverGol Prime } 0.32 \mathrm{oz} / \mathrm{cwt}\end{array}$ & -- \\
\hline 3 & 3 & Aeris Seed Applied System $0.75 \mathrm{mg}$ ai/seed & -- \\
\hline 4 & 4 & Aeris Seed Applied System $0.75 \mathrm{mg}$ ai/seed & -- \\
\hline 5 & 3 & $\begin{array}{l}\text { Spera } 1.8 \mathrm{oz}+\text { Vortex } 0.08 \mathrm{oz}+\text { Allegiance FL } 0.75 \mathrm{oz} \\
+ \text { EverGol Prime } 0.32 \mathrm{oz} / \mathrm{cwt}+\text { Aeris Seed Applied System } 0.75 \mathrm{mg} \\
\text { ai/seed }\end{array}$ & -- \\
\hline 6 & 4 & $\begin{array}{l}\text { Spera } 1.8 \mathrm{oz}+\text { Vortex } 0.08 \mathrm{oz}+\text { Allegiance FL } 0.75 \mathrm{oz} \\
+ \text { EverGol Prime } 0.32 \mathrm{oz} / \mathrm{cwt}+\text { Aeris Seed Applied System } 0.75 \mathrm{mg} \\
\text { ai/seed }\end{array}$ & -- \\
\hline 7 & 3 & $\begin{array}{l}\text { Spera } 1.8 \mathrm{oz}+\text { Vortex } 0.08 \mathrm{oz}+\text { Allegiance FL } 1.5 \mathrm{oz} \\
+ \text { EverGol Xtend }(\text { Prem \#1) } 0.64 \mathrm{oz} / \mathrm{cwt}\end{array}$ & -- \\
\hline 8 & 4 & $\begin{array}{l}\text { Spera } 1.8 \mathrm{oz}+\text { Vortex } 0.08 \mathrm{oz}+\text { Allegiance FL } 1.5 \mathrm{oz} \\
+ \text { EverGol Xtend }(\text { Prem \#1) } 0.64 \mathrm{oz} / \mathrm{cwt}\end{array}$ & -- \\
\hline 9 & 3 & $\begin{array}{l}\text { Spera } 1.8 \mathrm{oz}+\text { Vortex } 0.08 \mathrm{oz}+\text { Allegiance FL } 1.5 \mathrm{oz} \\
+ \text { EverGol Xtend (Prem \#1) } 0.64 \mathrm{oz} / \mathrm{cwt}+\text { Aeris Seed Applied System } \\
0.75 \mathrm{mg} \mathrm{ai} / \mathrm{seed}\end{array}$ & -- \\
\hline 10 & 4 & $\begin{array}{l}\text { Spera } 1.8 \mathrm{oz}+\text { Vortex } 0.08 \mathrm{oz}+\text { Allegiance FL } 1.5 \mathrm{oz} \\
+ \text { EverGol Xtend (Prem \#1) } 0.64 \mathrm{oz} / \mathrm{cwt}+\text { Aeris Seed Applied System } \\
0.75 \mathrm{mg} \mathrm{ai} / \mathrm{seed}\end{array}$ & -- \\
\hline 11 & 3 & $\begin{array}{l}\text { Spera } 1.8 \mathrm{oz}+\text { Vortex } 0.08 \mathrm{oz}+\text { Allegiance FL } 0.75 \mathrm{oz} \\
+ \text { EverGol Prime } 0.32 \mathrm{oz} / \mathrm{cwt}+\text { Vibrance CST (Prem \#2) } 4.08 \mathrm{mg} \\
\text { ai/seed }\end{array}$ & - - \\
\hline 12 & 4 & $\begin{array}{l}\text { Spera } 1.8 \mathrm{oz}+\text { Vortex } 0.08 \mathrm{oz}+\text { Allegiance FL } 0.75 \mathrm{oz} \\
+ \text { EverGol Prime } 0.32 \mathrm{oz} / \mathrm{cwt}+\text { Vibrance CST (Prem \#2) } 4.08 \mathrm{mg} \\
\text { ai/seed }\end{array}$ & -- \\
\hline 13 & 3 & $\begin{array}{l}\text { Spera } 1.8 \mathrm{oz}+\text { Vortex } 0.08 \mathrm{oz}+\text { Allegiance FL } 0.75 \mathrm{oz} \\
+ \text { EverGol Prime } 0.32 \mathrm{oz} / \mathrm{cwt}\end{array}$ & $\begin{array}{l}\text { Azoxystrobin (Quadris) } \\
6 \mathrm{fl} \mathrm{oz}\end{array}$ \\
\hline 14 & 4 & $\begin{array}{l}\text { Spera } 1.8 \mathrm{oz}+\text { Vortex } 0.08 \mathrm{oz}+\text { Allegiance FL } 0.75 \mathrm{oz} \\
+ \text { EverGol Prime } 0.32 \mathrm{oz} / \mathrm{cwt}\end{array}$ & $\begin{array}{l}\text { Azoxystrobin (Quadris) } \\
6 \mathrm{fl} \mathrm{oz}\end{array}$ \\
\hline
\end{tabular}


SOIL TYPE: Kenansville loamy fine sand

SOIL FERTILITY REPORT (Jan 2020):

\begin{tabular}{|l|l|l|l|l|l|l|l|l|l|}
\hline $\mathbf{p H}$ & $\mathbf{P}(\mathbf{l b} / \mathbf{A})$ & $\mathbf{K}(\mathbf{l b} / \mathbf{A})$ & $\mathbf{C a}(\mathbf{l b} / \mathbf{A})$ & $\mathbf{M g}(\mathbf{l b} / \mathbf{A})$ & $\mathbf{Z n}(\mathbf{p p m})$ & $\mathbf{M n}(\mathbf{p p m})$ & $\mathbf{C u}(\mathbf{p p m})$ & $\mathbf{F e}(\mathbf{p p m})$ & $\mathbf{B}(\mathbf{p p m})$ \\
\hline 6.3 & 110 & 240 & 1266 & 91 & 0.6 & 5.8 & 0.4 & 26.1 & 0.2 \\
\hline
\end{tabular}

MAINTENANCE CHEMICAL PROGRAMS:

\begin{tabular}{|l|l|}
\hline Fertilizer & Standard \\
\hline Herbicides & Standard \\
\hline Insecticides & Standard \\
\hline Fungicides & None except treatments \\
\hline Nematicides & Standard \\
\hline
\end{tabular}

MAINTENANCE CHEMICAL APPLICATIONS:

\begin{tabular}{|l|l|l|l|}
\hline Date & Type and target & Product and formulation & Rate/A \\
\hline $3 / 19 / 20$ & Herbicide & $2,4-\mathrm{D}$ & $1 \mathrm{pt}$ \\
\hline $3 / 19 / 20$ & Herbicide & Panther & $2 \mathrm{fl} \mathrm{oz}$ \\
\hline $5 / 4 / 20$ & Fertility & $10-10-28$ & $285 \mathrm{lbs}$ \\
\hline $5 / 17 / 20$ & Herbicide & Prowl H2O & $1 \mathrm{pt}$ \\
\hline $6 / 5 / 20$ & Herbicide & Roundup WeatherMAX & $22 \mathrm{fl} \mathrm{oz}$ \\
\hline $6 / 12 / 20$ & Herbicide & Roundup WeatherMAX & $20 \mathrm{fl} \mathrm{oz}$ \\
\hline $6 / 12 / 20$ & Insecticide & Orthene $75 \mathrm{~S}$ & $12 \mathrm{oz}$ \\
\hline $7 / 8 / 20$ & Fertility & $24-0-0-3$ & $80 \mathrm{units}$ \\
\hline $7 / 23 / 20$ & Insecticide & Transform WG & $2.45 \mathrm{oz}$ \\
\hline $8 / 10 / 20$ & Plant growth regulator & Pentia & $16 \mathrm{fl} \mathrm{oz}$ \\
\hline $8 / 10 / 20$ & Herbicide & Roundup WeatherMAX & $24 \mathrm{fl} \mathrm{oz}$ \\
\hline $8 / 10 / 20$ & Insecticide & Besiege & $12.8 \mathrm{fl} \mathrm{oz}$ \\
\hline $8 / 10 / 20$ & Insecticide & Bifenthrin & $6 \mathrm{fl} \mathrm{oz}$ \\
\hline $10 / 21 / 20$ & Defoliant/boll opener & Finish 6 Pro & $1 \mathrm{qt}$ \\
\hline $10 / 21 / 20$ & Defoliant/boll opener & Folex 6 EC & $4 \mathrm{fl} \mathrm{oz}$ \\
\hline $10 / 21 / 20$ & Defoliant/boll opener & Dropp SC & $3 \mathrm{fl} \mathrm{oz}$ \\
\hline
\end{tabular}


Table 1. Effect of seeding rate and fungicide seed treatment on emergence and thrips injury in cotton (COTSEEDFUN120, Suffolk, VA 2020).

\begin{tabular}{|c|c|c|c|c|c|}
\hline \multirow{2}{*}{$\begin{array}{l}\text { Seeding } \\
\text { rate } \\
\text { /row ft }\end{array}$} & \multirow[b]{2}{*}{ Treatment and rate re $^{\mathrm{z}}$} & \multicolumn{2}{|c|}{ Plants/ft $\mathbf{t}^{\mathbf{y}}$} & \multicolumn{2}{|c|}{ Thrips injury $(0-5)^{x}$} \\
\hline & & 29 May & 11 Jun & 4 Jun & 10 Jun \\
\hline 1. 3 seed & $\begin{array}{l}\text { Spera } 1.8 \mathrm{oz}+\text { Vortex } 0.08 \mathrm{oz} \\
+ \text { Allegiance FL } 0.75 \mathrm{oz}+\text { EverGol Prime } 0.32 \mathrm{oz} / \mathrm{cwt}(\mathrm{S})\end{array}$ & $2.0 \mathrm{~cd}$ & $2.0 \mathrm{de}$ & $4.4 \mathrm{a}$ & $4.3 \mathrm{a}$ \\
\hline 2. 4 seed & $\begin{array}{l}\text { Spera } 1.8 \mathrm{oz}+\text { Vortex } 0.08 \mathrm{oz} \\
+ \text { Allegiance FL } 0.75 \mathrm{oz}+\text { EverGol Prime } 0.32 \mathrm{oz} / \mathrm{cwt}(\mathrm{S})\end{array}$ & $2.2 \mathrm{c}$ & $2.2 \mathrm{~d}$ & $3.0 \mathrm{~b}$ & $3.6 \mathrm{~b}-\mathrm{c}$ \\
\hline 3. 3 seed & Aeris Seed Applied System 0.75 mg ai/seed (S) & $2.1 \mathrm{~cd}$ & $2.1 \mathrm{~d}$ & $3.3 \mathrm{~b}$ & $3.4 \mathrm{~d}-\mathrm{f}$ \\
\hline 4. 4 seed & Aeris Seed Applied System $0.75 \mathrm{mg}$ ai/seed (S) & $3.2 \mathrm{ab}$ & $3.2 \mathrm{ab}$ & $3.9 \mathrm{a}$ & $4.0 \mathrm{a}-\mathrm{c}$ \\
\hline 5. 3 seed & $\begin{array}{l}\text { Spera } 1.8 \mathrm{oz}+\text { Vortex } 0.08 \mathrm{oz}+\text { Allegiance FL } 0.75 \mathrm{oz}+ \\
\text { EverGol Prime } 0.32 \mathrm{oz} / \mathrm{cwt}+\text { Aeris Seed Applied System } \\
0.75 \mathrm{mg} \mathrm{ai} / \mathrm{seed}(\mathrm{S})\end{array}$ & $2.0 \mathrm{~cd}$ & $2.1 \mathrm{de}$ & $3.1 \mathrm{~b}$ & $3.1 \mathrm{~d}-\mathrm{f}$ \\
\hline 6. 4 seed & $\begin{array}{l}\text { Spera } 1.8 \mathrm{oz}+\text { Vortex } 0.08 \mathrm{oz}+\text { Allegiance FL } 0.75 \mathrm{oz}+ \\
\text { EverGol Prime } 0.32 \mathrm{oz} / \mathrm{cwt}+\text { Aeris Seed Applied System } \\
0.75 \mathrm{mg} \mathrm{ai} / \mathrm{seed}(\mathrm{S})\end{array}$ & $2.9 \mathrm{~b}$ & $2.8 \mathrm{c}$ & $2.8 \mathrm{~b}$ & $2.9 \mathrm{f}$ \\
\hline 7. 3 seed & $\begin{array}{l}\text { Spera } 1.8 \mathrm{oz}+\text { Vortex } 0.08 \mathrm{oz}+\text { Allegiance FL } 1.5 \mathrm{oz}+ \\
\text { EverGol Xtend (Prem \#1) } 0.64 \mathrm{oz} / \mathrm{cwt}(\mathrm{S})\end{array}$ & $1.9 \mathrm{~cd}$ & $1.9 \mathrm{de}$ & $3.0 \mathrm{~b}$ & $3.1 \mathrm{~d}-\mathrm{f}$ \\
\hline 8.4 seed & $\begin{array}{l}\text { Spera } 1.8 \mathrm{oz}+\text { Vortex } 0.08 \mathrm{oz}+\text { Allegiance FL } 1.5 \mathrm{oz}+ \\
\text { EverGol Xtend (Prem \#1) } 0.64 \mathrm{oz} / \mathrm{cwt}(\mathrm{S})\end{array}$ & $3.4 \mathrm{a}$ & $3.3 \mathrm{a}$ & $3.9 \mathrm{a}$ & $4.1 \mathrm{ab}$ \\
\hline 9.3 seed & $\begin{array}{l}\text { Spera } 1.8 \mathrm{oz}+\text { Vortex } 0.08 \mathrm{oz}+\text { Allegiance FL } 1.5 \mathrm{oz}+ \\
\text { EverGol Xtend (Prem \#1) } 0.64 \mathrm{oz} / \mathrm{cwt}+\text { Aeris Seed } \\
\text { Applied System } 0.75 \mathrm{mg} \mathrm{ai} / \mathrm{seed}(\mathrm{S})\end{array}$ & $1.7 \mathrm{~d}$ & $1.8 \mathrm{e}$ & $3.1 \mathrm{~b}$ & $3.5 \mathrm{c}-\mathrm{e}$ \\
\hline 10. 4 seed & $\begin{array}{l}\text { Spera } 1.8 \mathrm{oz}+\text { Vortex } 0.08 \mathrm{oz}+\text { Allegiance FL } 1.5 \mathrm{oz}+ \\
\text { EverGol Xtend (Prem \#1) } 0.64 \mathrm{oz} / \mathrm{cwt}+\text { Aeris Seed } \\
\text { Applied System } 0.75 \mathrm{mg} \mathrm{ai} / \mathrm{seed}(\mathrm{S})\end{array}$ & $3.0 \mathrm{ab}$ & $3.0 \mathrm{bc}$ & $2.8 \mathrm{~b}$ & $2.9 \mathrm{f}$ \\
\hline 11. 3 seed & $\begin{array}{l}\text { Spera } 1.8 \mathrm{oz}+\text { Vortex } 0.08 \mathrm{oz}+\text { Allegiance FL } 0.75 \mathrm{oz}+ \\
\text { EverGol Prime } 0.32 \mathrm{oz} / \mathrm{cwt}+\text { Vibrance CST }(\text { Prem \#2) } \\
4.08 \mathrm{mg} \text { ai/seed }(\mathrm{S}) \\
\text { Quadris } 6 \mathrm{fl} \mathrm{oz} / \mathrm{A}(\mathrm{F})\end{array}$ & $2.3 \mathrm{c}$ & $2.0 \mathrm{de}$ & $4.1 \mathrm{a}$ & $4.1 \mathrm{ab}$ \\
\hline 12. 4 seed & $\begin{array}{l}\text { Spera } 1.8 \mathrm{oz}+\text { Vortex } 0.08 \mathrm{oz}+\text { Allegiance FL } 0.75 \mathrm{oz}+ \\
\text { EverGol Prime } 0.32 \mathrm{oz} / \mathrm{cwt}+\text { Vibrance CST (Prem \#2) } \\
4.08 \mathrm{mg} \text { ai/seed }(\mathrm{S}) \\
\text { Quadris } 6 \mathrm{fl} \mathrm{oz} / \mathrm{A}(\mathrm{F})\end{array}$ & $3.0 \mathrm{ab}$ & $3.1 \mathrm{a}-\mathrm{c}$ & $3.0 \mathrm{~b}$ & $3.0 \mathrm{ef}$ \\
\hline 13. 3 seed & $\begin{array}{l}\text { Spera } 1.8 \mathrm{oz}+\text { Vortex } 0.08 \mathrm{oz}+\text { Allegiance FL } 0.75 \mathrm{oz}+ \\
\text { EverGol Prime } 0.32 \mathrm{oz} / \mathrm{cwt}(\mathrm{S}) \\
+ \text { Quadris } 6 \mathrm{fl} \mathrm{oz} / \mathrm{A}(\mathrm{F})\end{array}$ & $2.0 \mathrm{~cd}$ & $1.9 \mathrm{de}$ & $3.3 \mathrm{~b}$ & $3.1 \mathrm{~d}-\mathrm{f}$ \\
\hline 14. 4 seed & $\begin{array}{l}\text { Spera } 1.8 \mathrm{oz}+\text { Vortex } 0.08 \mathrm{oz}+\text { Allegiance FL } 0.75 \mathrm{oz}+ \\
\text { EverGol Prime } 0.32 \mathrm{oz} / \mathrm{cwt}(\mathrm{S}) \\
+ \text { Quadris } 6 \mathrm{fl} \mathrm{oz} / \mathrm{A}(\mathrm{F})\end{array}$ & $2.9 \mathrm{~b}$ & $3.0 \mathrm{a}-\mathrm{c}$ & $2.9 \mathrm{~b}$ & $3.0 \mathrm{ef}$ \\
\hline$P(\mathrm{~F})$ & & $<0.0001$ & $<0.0001$ & $<0.0001$ & $<0.0001$ \\
\hline LSD & & 0.41 & 0.35 & 0.51 & 0.51 \\
\hline
\end{tabular}

z $(S)=$ seed treatment; $(F)=$ in furrow at planting (trts. $13 \&$ 14). Seed was planted 15 May.

y Determined from counts in two, 30 -ft rows per plot.

$x$ Thrips injury rating scale: $0=$ no damage, $5=$ dead plants.

Means in a column followed by the same letter(s) are not significantly different according to Fisher's Protected LSD $(P=0.05)$. 
Table 2. Effect of seeding rate and fungicide seed treatment on plant health and yield in cotton (COTSEEDFUN120, Suffolk, VA 2020).

\begin{tabular}{|c|c|c|c|c|}
\hline \multirow{2}{*}{$\begin{array}{l}\text { Seeding } \\
\text { rate/row ft }\end{array}$} & \multirow[b]{2}{*}{ Treatment and rate ${ }^{\mathrm{z}}$} & \multirow{2}{*}{$\begin{array}{l}\text { Vigor, 1-10 } \\
22 \text { Jun }\end{array}$} & \multicolumn{2}{|c|}{ Yield $^{\mathrm{x}}$} \\
\hline & & & $\mathbf{l b} / \mathbf{A}$ & bales/A \\
\hline 1. 3 seed & $\begin{array}{l}\text { Spera } 1.8 \mathrm{oz}+\text { Vortex } 0.08 \mathrm{oz} \\
+ \text { Allegiance FL } 0.75 \mathrm{oz}+\text { EverGol Prime } 0.32 \mathrm{oz} / \mathrm{cwt}(\mathrm{S})\end{array}$ & $6.6 \mathrm{~d}$ & 3588 & 3.6 \\
\hline 2. 4 seed & $\begin{array}{l}\text { Spera } 1.8 \mathrm{oz}+\text { Vortex } 0.08 \mathrm{oz} \\
+ \text { Allegiance FL } 0.75 \mathrm{oz}+\text { EverGol Prime } 0.32 \mathrm{oz} / \mathrm{cwt}(\mathrm{S})\end{array}$ & $9.1 \mathrm{ab}$ & 3591 & 3.5 \\
\hline 3. 3 seed & Aeris Seed Applied System $0.75 \mathrm{mg}$ ai/seed (S) & $9.4 \mathrm{a}$ & 3524 & 3.5 \\
\hline 4. 4 seed & Aeris Seed Applied System $0.75 \mathrm{mg}$ ai/seed (S) & $8.4 \mathrm{bc}$ & 3476 & 3.6 \\
\hline 5. 3 seed & $\begin{array}{l}\text { Spera } 1.8 \mathrm{oz}+\text { Vortex } 0.08 \mathrm{oz}+\text { Allegiance FL } 0.75 \mathrm{oz}+ \\
\text { EverGol Prime } 0.32 \mathrm{oz} / \mathrm{cwt}+\text { Aeris Seed Applied System } \\
0.75 \mathrm{mg} \text { ai/seed }(\mathrm{S})\end{array}$ & $9.5 \mathrm{a}$ & 3494 & 3.5 \\
\hline 6. 4 seed & $\begin{array}{l}\text { Spera } 1.8 \mathrm{oz}+\text { Vortex } 0.08 \mathrm{oz}+\text { Allegiance FL } 0.75 \mathrm{oz}+ \\
\text { EverGol Prime } 0.32 \mathrm{oz} / \mathrm{cwt}+\text { Aeris Seed Applied System } \\
0.75 \mathrm{mg} \text { ai/seed }(\mathrm{S})\end{array}$ & $9.6 \mathrm{a}$ & 3594 & 3.7 \\
\hline 7. 3 seed & $\begin{array}{l}\text { Spera } 1.8 \mathrm{oz}+\text { Vortex } 0.08 \mathrm{oz}+\text { Allegiance FL } 1.5 \mathrm{oz}+ \\
\text { EverGol Xtend (Prem \#1) } 0.64 \mathrm{oz} / \mathrm{cwt}(\mathrm{S})\end{array}$ & $9.1 \mathrm{ab}$ & 3536 & 3.6 \\
\hline 8. 4 seed & $\begin{array}{l}\text { Spera } 1.8 \mathrm{oz}+\text { Vortex } 0.08 \mathrm{oz}+\text { Allegiance FL } 1.5 \mathrm{oz}+ \\
\text { EverGol Xtend (Prem \#1) } 0.64 \mathrm{oz} / \mathrm{cwt}(\mathrm{S})\end{array}$ & $7.9 \mathrm{c}$ & 3303 & 3.3 \\
\hline 9. 3 seed & $\begin{array}{l}\text { Spera } 1.8 \mathrm{oz}+\text { Vortex } 0.08 \mathrm{oz}+\text { Allegiance FL } 1.5 \mathrm{oz}+ \\
\text { EverGol Xtend (Prem \#1) } 0.64 \mathrm{oz} / \mathrm{cwt}+\text { Aeris Seed Applied } \\
\text { System } 0.75 \mathrm{mg} \text { ai/seed (S) }\end{array}$ & $9.3 \mathrm{ab}$ & 3258 & 3.3 \\
\hline 10. 4 seed & $\begin{array}{l}\text { Spera } 1.8 \mathrm{oz}+\text { Vortex } 0.08 \mathrm{oz}+\text { Allegiance FL } 1.5 \mathrm{oz}+ \\
\text { EverGol Xtend (Prem \#1) } 0.64 \mathrm{oz} / \mathrm{cwt}+\text { Aeris Seed Applied } \\
\text { System } 0.75 \mathrm{mg} \text { ai/seed (S) }\end{array}$ & $9.6 \mathrm{a}$ & 3672 & 3.6 \\
\hline 11. 3 seed & $\begin{array}{l}\text { Spera } 1.8 \mathrm{oz}+\text { Vortex } 0.08 \mathrm{oz}+\text { Allegiance FL } 0.75 \mathrm{oz}+ \\
\text { EverGol Prime } 0.32 \mathrm{oz} / \mathrm{cwt}+\text { Vibrance CST (Prem \#2) } 4.08 \\
\mathrm{mg} \text { ai/seed }(\mathrm{S}) \\
\text { Quadris } 6 \mathrm{fl} \mathrm{oz/A} \mathrm{(F)}\end{array}$ & $7.9 \mathrm{c}$ & 3612 & 3.5 \\
\hline 12. 4 seed & $\begin{array}{l}\text { Spera } 1.8 \mathrm{oz}+\text { Vortex } 0.08 \mathrm{oz}+\text { Allegiance FL } 0.75 \mathrm{oz}+ \\
\text { EverGol Prime } 0.32 \mathrm{oz} / \mathrm{cwt}+\text { Vibrance CST }(\text { Prem \#2) } 4.08 \\
\mathrm{mg} \text { ai/seed }(\mathrm{S}) \\
\text { Quadris } 6 \mathrm{fl} \mathrm{oz} / \mathrm{A}(\mathrm{F})\end{array}$ & $9.8 \mathrm{a}$ & 3706 & 3.7 \\
\hline 13. 3 seed & $\begin{array}{l}\text { Spera } 1.8 \mathrm{oz}+\text { Vortex } 0.08 \mathrm{oz}+\text { Allegiance FL } 0.75 \mathrm{oz}+ \\
\text { EverGol Prime } 0.32 \mathrm{oz} / \mathrm{cwt}(\mathrm{S}) \\
+ \text { Quadris } 6 \mathrm{fl} \mathrm{oz} / \mathrm{A}(\mathrm{F})\end{array}$ & $9.6 \mathrm{a}$ & 3346 & 3.4 \\
\hline 14. 4 seed & $\begin{array}{l}\text { Spera } 1.8 \mathrm{oz}+\text { Vortex } 0.08 \mathrm{oz}+\text { Allegiance FL } 0.75 \mathrm{oz}+ \\
\text { EverGol Prime } 0.32 \mathrm{oz} / \mathrm{cwt}(\mathrm{S}) \\
+ \text { Quadris } 6 \mathrm{fl} \mathrm{oz} / \mathrm{A}(\mathrm{F})\end{array}$ & $9.9 \mathrm{a}$ & 3506 & 3.5 \\
\hline$P(\mathrm{~F})$ & & $<0.0001$ & 0.96 & 0.94 \\
\hline LSD & & 0.98 & N.S. & N.S. \\
\hline
\end{tabular}

$\mathrm{z}(\mathrm{S})=$ seed treatment; $(\mathrm{F})=$ in furrow at planting trts. $13 \& 14)$. Seed was planted 15 May.

y Vigor index rating scale: $10=100 \%$ vigor, $1=$ no vigor.

$x$ Weight (lb/A) includes lint + seed, bales/A are weight of lint only. Lint weight $(480 \mathrm{lb} / \mathrm{b}$ ale $)$ was determined by ginning samples of seed cotton from each treatment. Plots were harvested on 3 Dec.

Means in a column followed by the same letter(s) are not significantly different according to Fisher's Protected LSD $(P=0.05)$. 
TEST ID: COTSEEDNEMA120

PURPOSE: To evaluate in-furrow and seed treatment nematicides for control of plant-parasitic nematodes in cotton and impact on yield

LOCATION: Tidewater Research Farm, Hare Rd., Suffolk, VA

CROP INFORMATION:

\begin{tabular}{|l|l|}
\hline Field & $16 \mathrm{~B}$ \\
\hline Trial GPA coordinates & $36.664085,-76.732527$ \\
\hline Crop history & 2019 peanut, 2018 corn, 2017 cotton \\
\hline Planting date & 15 May \\
\hline Variety & DP $1646 \mathrm{~B} 2 \mathrm{XF}$ \\
\hline Seeding rate & $3.5-4$ seed/row ft \\
\hline Plot length/width & 30 \\
\hline Number of rows & 2 \\
\hline Row spacing & $36{ }^{\prime}$ \\
\hline Alleys (length between blocks) & $8^{\prime}$ \\
\hline Harvest date & $3 \mathrm{Dec}$ \\
\hline
\end{tabular}

EXPERIMENTAL DESIGN: Randomized complete block with four replicates

TREATMENT APPLICATION:

\begin{tabular}{|l|l|l|}
\hline & IF liquid & IF granular \\
\hline Equipment & --- & Nobel box \\
\hline Pressure (psi) & & --- \\
\hline Nozzle type & .075 microtube & --- \\
\hline Volume (gal/A) & $5 \mathrm{gal} / \mathrm{A}$ & $\mathrm{lb} / \mathrm{A}$ (trt rate) \\
\hline
\end{tabular}

TREATMENTS:

\begin{tabular}{|l|l|l|}
\hline Trt \# & Seed treatment and rate & In-furrow treatment and rate/A \\
\hline 1 & Base Fungicide, non-nematicide control & - - \\
\hline 2 & CoPEO Prime $0.2 \mathrm{mg}$ ai/seed & -- \\
\hline 3 & BioST Nematicide $1007.2 \mathrm{oz} / \mathrm{cwt}$ & -- \\
\hline 4 & Base Fungicide, non-nematicide control & Velum Prime $500 \mathrm{SC} 6.5 \mathrm{fl} \mathrm{oz}$ \\
\hline 5 & Base Fungicide, non-nematicide control & Propulse $480 \mathrm{SC} 13.6 \mathrm{fl} \mathrm{oz}$ \\
\hline 6 & CoPEO Prime $0.2 \mathrm{mg}$ ai/seed & Velum Prime $500 \mathrm{SC} 6 \mathrm{fl} \mathrm{oz}$ \\
\hline 7 & CoPEO Prime $0.2 \mathrm{mg}$ ai/seed & Propulse $480 \mathrm{SC} 13.6 \mathrm{fl} \mathrm{oz}$ \\
\hline
\end{tabular}

SOIL TYPE: Kenansville loamy fine sand

SOIL FERTILITY REPORT (Jan 2020):

\begin{tabular}{|l|l|l|l|l|l|l|l|l|l|}
\hline $\mathbf{p H}$ & $\mathbf{P}(\mathbf{l b} / \mathbf{A})$ & $\mathbf{K}(\mathbf{l b} / \mathbf{A})$ & $\mathbf{C a}(\mathbf{l b} / \mathbf{A})$ & $\mathbf{M g}(\mathbf{l b} / \mathbf{A})$ & $\mathbf{Z n}(\mathbf{p p m})$ & $\mathbf{M n}(\mathbf{p p m})$ & $\mathbf{C u}(\mathbf{p p m})$ & $\mathbf{F e}(\mathbf{p p m})$ & $\mathbf{B}(\mathbf{p p m})$ \\
\hline 6.3 & 110 & 240 & 1266 & 91 & 0.6 & 5.8 & 0.4 & 26.1 & 0.2 \\
\hline
\end{tabular}


MAINTENANCE CHEMICAL PROGRAMS:

\begin{tabular}{|l|l|}
\hline Fertilizer & Standard \\
\hline Herbicides & Standard \\
\hline Insecticides & Standard \\
\hline Fungicides & None except treatments \\
\hline Nematicides & None except treatments \\
\hline
\end{tabular}

MAINTENANCE CHEMICAL PROGRAMS:

\begin{tabular}{|l|l|l|l|}
\hline Date & Type and target & Product and formulation & Rate/A \\
\hline $3 / 19 / 20$ & Herbicide & $2,4-D$ & $1 \mathrm{pt}$ \\
\hline $3 / 19 / 20$ & Herbicide & Panther & $2 \mathrm{fl} \mathrm{oz}$ \\
\hline $5 / 4 / 20$ & Fertility & $10-10-28$ & $285 \mathrm{lbs}$ \\
\hline $5 / 17 / 20$ & Herbicide & Prowl H2O & $1 \mathrm{pt}$ \\
\hline $6 / 4 / 20$ & Insecticide & Orthene $75 \mathrm{~S}$ & $12 \mathrm{oz}$ \\
\hline $6 / 5 / 20$ & Herbicide & Roundup WeatherMAX & $22 \mathrm{fl} \mathrm{oz}$ \\
\hline $6 / 12 / 20$ & Herbicide & Roundup WeatherMAX & $20 \mathrm{fl} \mathrm{oz}$ \\
\hline $6 / 12 / 20$ & Insecticide & Orthene 75 S & $12 \mathrm{oz}$ \\
\hline $7 / 8 / 20$ & Fertility & $24-0-0-3$ & $80 \mathrm{units}$ \\
\hline $7 / 23 / 20$ & Insecticide & Transform WG & $2.45 \mathrm{oz}$ \\
\hline $8 / 10 / 20$ & Plant growth regulator & Pentia & $16 \mathrm{fl} \mathrm{oz}$ \\
\hline $8 / 10 / 20$ & Herbicide & Roundup WeatherMAX & $24 \mathrm{fl} \mathrm{oz}$ \\
\hline $8 / 10 / 20$ & Insecticide & Besiege & $12.8 \mathrm{fl} \mathrm{oz}$ \\
\hline $8 / 10 / 20$ & Insecticide & Bifenthrin & $6 \mathrm{fl} \mathrm{oz}$ \\
\hline $10 / 21 / 20$ & Defoliant/boll opener & Finish 6 Pro & $1 \mathrm{qt}$ \\
\hline $10 / 21 / 20$ & Defoliant/boll opener & Folex 6 EC & $4 \mathrm{fl} \mathrm{oz}$ \\
\hline $10 / 21 / 20$ & Defoliant/boll opener & Dropp SC & $3 \mathrm{fl} \mathrm{oz}$ \\
\hline
\end{tabular}


Table 1. Effect of fungicide treatment on emergence and plant health in cotton (COTSEEDNEMA120, Suffolk, VA 2020).

\begin{tabular}{|c|c|c|c|}
\hline Seed treatment ${ }^{\mathrm{z}}$ & In-furrow rate/ $/ A^{z}$ & $\begin{array}{c}\text { Plants/ft. } \\
\text { 11 Jun }\end{array}$ & $\begin{array}{c}\text { Vigor } \\
(1-6)^{x} \\
12 \text { Jun } \\
\end{array}$ \\
\hline 1. Base fungicide, non-nematicide control & - & $3.2 \mathrm{a}$ & 5.5 \\
\hline 2. CoPEO Prime $0.2 \mathrm{mg}$ ai $/ \mathrm{seed}$ & - & $2.2 \mathrm{~b}$ & 5.5 \\
\hline 3. BioST Nematicide $1007.2 \mathrm{oz} / \mathrm{cwt}$ & - & $2.4 \mathrm{~b}$ & 4.5 \\
\hline 4. Base fungicide, non-nematicide control & Velum Prime $500 \mathrm{SC} 6.5 \mathrm{fl} \mathrm{oz}$ & $2.9 \mathrm{a}$ & 5.4 \\
\hline 5. Base fungicide, non-nematicide control & Propulse 480 SC $13.6 \mathrm{fl} \mathrm{oz}$ & $3.2 \mathrm{a}$ & 5.5 \\
\hline 6. CoPEO Prime $0.2 \mathrm{mg}$ ai $/ \mathrm{seed}$ & Velum Prime $500 \mathrm{SC} 6.5 \mathrm{fl} \mathrm{oz}$ & $1.6 \mathrm{c}$ & 5.4 \\
\hline 7. CoPEO Prime $0.2 \mathrm{mg}$ ai $/ \mathrm{seed}$ & Propulse $480 \mathrm{SC} 13.6 \mathrm{fl} \mathrm{oz}$ & $1.6 \mathrm{c}$ & 5.0 \\
\hline$P(\mathrm{~F})$ & & $<0.0001$ & 0.23 \\
\hline LSD & & 0.49 & N.S. \\
\hline
\end{tabular}

z (F) In-furrow at planting 9 May; seed treatments were applied by personnel with Bayer CropScience.

y Determined from counts in two, 30 -ft rows per plot.

$x$ Vigor index rating scale: $6=100 \%$ vigor, $1=$ no vigor.

Means followed by the same letter(s) in a column are not significantly different according to Fisher's Protected LSD ( $\mathrm{P}=0.05)$.

Table 2. Mid-season nematode populations in composite samples by treatment (COTSEEDNEMA120, Suffolk, VA 2020).

\begin{tabular}{|c|c|c|c|}
\hline \multirow[b]{2}{*}{ Seed treatment ${ }^{\mathrm{z}}$} & \multirow[b]{2}{*}{ In-furrow rate/A ${ }^{z}$} & \multicolumn{2}{|c|}{$\begin{array}{c}\text { Nematodes /500 cc soil } \\
\text { 8 Jul }\end{array}$} \\
\hline & & Root knot & Stubby root \\
\hline 1. Base fungicide, non-nematicide control & -- & 1060 & 0 \\
\hline 2. CoPEO Prime $0.2 \mathrm{mg}$ ai $/ \mathrm{seed}$ & -- & 340 & 60 \\
\hline 3. BioST Nematicide $1007.2 \mathrm{oz} / \mathrm{cwt}$ & -- & 520 & 20 \\
\hline 4. Base fungicide, non-nematicide control & Velum Prime $500 \mathrm{SC} 6.5 \mathrm{fl} \mathrm{oz}$ & 200 & 40 \\
\hline 5. Base fungicide, non-nematicide control & Propulse $480 \mathrm{SC} 13.6 \mathrm{fl} \mathrm{oz}$ & 380 & 60 \\
\hline 6. CoPEO Prime $0.2 \mathrm{mg}$ ai/seed & Velum Prime $500 \mathrm{SC} 6.5 \mathrm{fl} \mathrm{oz}$ & 280 & 20 \\
\hline 7. CoPEO Prime $0.2 \mathrm{mg}$ ai $/ \mathrm{seed}$ & Propulse 480 SC $13.6 \mathrm{fl} \mathrm{oz}$ & 340 & 40 \\
\hline
\end{tabular}

z (F) In-furrow at planting 9 May; seed treatments were applied by personnel with Bayer CropScience.

y A pre-plant soil composite was sampled from four untreated areas of trial area and tested for nematode populations on 9 May. Number nematodes $/ 500 \mathrm{cc}$ soil were as follows: 1840 root knot. For mid-season sampling on 8 Jul, a composite sample was taken from 4 reps of each treatment and counted. Roots were observed for galling at harvest, no galling was observed. 
Table 3. Effect of fungicide treatment on yield in cotton (COTSEEDNEMA120, Suffolk, VA 2020 ).

\begin{tabular}{|c|c|c|c|}
\hline \multirow[b]{2}{*}{ Seed treatment ${ }^{\mathrm{z}}$} & \multirow[b]{2}{*}{ In-furrow rate/ $\mathbf{A}^{\mathrm{z}}$} & \multicolumn{2}{|c|}{ Yield $^{\mathrm{x}}$} \\
\hline & & $\mathbf{l b} / \mathbf{A}$ & bales/A \\
\hline 1. Base fungicide, non-nematicide control & -- & 3458 & 3.4 \\
\hline 2. CoPEO Prime $0.2 \mathrm{mg}$ ai $/ \mathrm{seed}$ & - & 3385 & 3.3 \\
\hline 3. BioST Nematicide $1007.2 \mathrm{oz} / \mathrm{cwt}$ & -- & 3446 & 3.5 \\
\hline 4. Base fungicide, non-nematicide control & Velum Prime $500 \mathrm{SC} 6.5 \mathrm{fl} \mathrm{oz}$ & 2962 & 2.9 \\
\hline 5. Base fungicide, non-nematicide control & Propulse $480 \mathrm{SC} 13.6 \mathrm{fl} \mathrm{oz}$ & 3098 & 3.1 \\
\hline 6. CoPEO Prime $0.2 \mathrm{mg}$ ai $/ \mathrm{seed}$ & Velum Prime $500 \mathrm{SC} 6.5 \mathrm{fl} \mathrm{oz}$ & 3724 & 3.7 \\
\hline 7. CoPEO Prime $0.2 \mathrm{mg}$ ai $/ \mathrm{seed}$ & Propulse 480 SC $13.6 \mathrm{fl} \mathrm{oz}$ & 3376 & 3.4 \\
\hline$P(\mathrm{~F})$ & & 0.34 & 0.34 \\
\hline LSD & & N.S. & N.S. \\
\hline
\end{tabular}

z (F) In-furrow at planting 9 May; Seed treatments were applied by personnel with Bayer CropScience.

$x$ Weight (lb/A) includes lint + seed, bales/A are weight of lint only. Lint weight $(480 \mathrm{lb} / \mathrm{bale})$ was determined by ginning samples of seed cotton from each treatment. Plots were harvested on 3 Dec. 
TEST ID: SOYFOLFUN120

PURPOSE: To compare fungicide treatments for foliar disease control of soybean and impact in yield

LOCATION: Tidewater AREC, 6321 Holland Rd., Suffolk, VA

CROP INFORMATION:

\begin{tabular}{|l|l|}
\hline Field & 56 \\
\hline Crop history & 2019 cotton, 2018 soybean, 2017 cotton \\
\hline Planting date & 27 May \\
\hline Variety & AG52X9 \\
\hline Seeding rate & 10 seed/row ft \\
\hline Plot length/width & $30^{\prime} \times 6^{\prime}$ \\
\hline Number of rows & 4 \\
\hline Row spacing & $366^{\prime}$ \\
\hline Alleys (length between blocks) & $8^{\prime}$ \\
\hline Harvest date & 9 Nov \\
\hline
\end{tabular}

EXPERIMENTAL DESIGN: Randomized complete block with four replicates

TREATMENT APPLICATION:

\begin{tabular}{|l|l|}
\hline Equipment & Lee Spider Sprayer \\
\hline Pressure (psi) & $38 \mathrm{psi}$ \\
\hline Nozzle type & Twinjet 8002VS \\
\hline Volume (gal/A) & 19.88 \\
\hline Surfactant & NIS $0.25 \%$ v/v (except trt 11) \\
\hline
\end{tabular}

TREATMENTS:

\begin{tabular}{|l|l|l|l|l|}
\hline Trt \# & Fungicide and formulation & Rate/A & App. timing & App. date \\
\hline 1 & Untreated control & & & \\
\hline 2 & Revytek SC & $8.0 \mathrm{fl} \mathrm{oz}$ & $\mathrm{R} 3$ & $28 \mathrm{Jul}$ \\
\hline & Priaxor 4.17 SC & $4.0 \mathrm{fl} \mathrm{oz}$ & & \\
+ Tilt 3.6 EC & $4.0 \mathrm{fl} \mathrm{oz}$ & $\mathrm{R} 3$ & $28 \mathrm{Jul}$ \\
\hline 4 & VeltyMA SC & $7.0 \mathrm{fl} \mathrm{oz}$ & $\mathrm{R} 3$ & $28 \mathrm{Jul}$ \\
\hline 5 & Miravis Top 1.67 SC & $13.7 \mathrm{fl} \mathrm{oz}$ & $\mathrm{R} 3$ & $28 \mathrm{Jul}$ \\
\hline 6 & Delaro 325 SC & $8.0 \mathrm{fl} \mathrm{oz}$ & $\mathrm{R} 3$ & $28 \mathrm{Jul}$ \\
\hline 7 & Quadris Top SBX 3.76 SC & $7.5 \mathrm{fl} \mathrm{oz}$ & $\mathrm{R} 3$ & $28 \mathrm{Jul}$ \\
\hline 8 & Domark 230 ME & $4.0 \mathrm{fl} \mathrm{oz}$ & $\mathrm{R} 3$ & 28 Jul \\
\hline 9 & Topguard EQ 4.29 SC & $5.0 \mathrm{fl} \mathrm{oz}$ & $\mathrm{R} 3$ & $28 \mathrm{Jul}$ \\
\hline 10 & Lucento 4.17 SC & $5.0 \mathrm{fl} \mathrm{oz}$ & $\mathrm{R} 3$ & $28 \mathrm{Jul}$ \\
\hline 11 & BS-CB & $10.0 \mathrm{fl} \mathrm{oz}$ & $\mathrm{R} 3$ & $28 \mathrm{Jul}$ \\
\hline 12 & Untreated control 2 & & & \\
\hline
\end{tabular}


SOIL TYPE: Rains fine sandy loam

SOIL FERTILITY REPORT (Jan 2020):

\begin{tabular}{|l|l|l|l|l|l|l|l|l|l|}
\hline $\mathbf{p H}$ & $\mathbf{P}(\mathbf{l b} / \mathbf{A})$ & $\mathbf{K}(\mathbf{l b} / \mathbf{A})$ & $\mathbf{C a}(\mathbf{l b} / \mathbf{A})$ & $\mathbf{M g}(\mathbf{l b} / \mathbf{A})$ & $\mathbf{Z n}(\mathbf{p p m})$ & $\mathbf{M n}(\mathbf{p p m})$ & $\mathbf{C u}(\mathbf{p p m})$ & $\mathbf{F e}(\mathbf{p p m})$ & $\mathbf{B}(\mathbf{p p m})$ \\
\hline 6.5 & 91 & 197 & 1380 & 152 & 0.7 & 5.0 & 0.5 & 34.2 & 0.2 \\
\hline
\end{tabular}

MAINTENANCE CHEMICAL PROGRAMS:

\begin{tabular}{|l|l|}
\hline Fertilizer & Standard \\
\hline Herbicides & Standard \\
\hline Insecticides & Standard \\
\hline Fungicides & None except treatments \\
\hline Nematicides & None \\
\hline
\end{tabular}

MAINTENANCE CHEMICAL APPLICATIONS:

\begin{tabular}{|l|l|l|l|}
\hline Date & Type and target & Product and formulation & Rate/A \\
\hline $6 / 11 / 20$ & Herbicide & Roundup WeatherMAX & $22 \mathrm{fl} \mathrm{oz}$ \\
\hline $8 / 6 / 20$ & Herbicide & Roundup WeatherMAX & $22 \mathrm{fl} \mathrm{oz}$ \\
\hline
\end{tabular}


Table 1. Effect of fungicide treatment on foliar disease in soybean (SOYFOLFUN120, Suffolk, VA 2020 ).

\begin{tabular}{|c|c|c|c|}
\hline \multirow[b]{2}{*}{ Treatment and rate $/ \mathrm{A}^{\mathrm{z}}$} & \multicolumn{2}{|c|}{$\begin{array}{c}\% \text { foliar disease }{ }^{\mathrm{y}} \\
24 \text { Sep }\end{array}$} & \multirow{2}{*}{$\begin{array}{c}\% \text { defoliation } \\
6 \text { Oct } \\
\end{array}$} \\
\hline & Cercospora blight & Anthracnose & \\
\hline 1. Untreated control & 1.0 & 5.5 & $47.5 \mathrm{a}$ \\
\hline 2. Revytek SC $8 \mathrm{fl} \mathrm{oz}$ & 1.0 & 4.8 & $16.3 \mathrm{e}$ \\
\hline $\begin{array}{l}\text { 3. Priaxor } 4.17 \mathrm{SC} 4 \mathrm{fl} \mathrm{oz} \\
+ \text { Tilt } 3.6 \mathrm{EC} 4 \mathrm{fl} \mathrm{oz}\end{array}$ & 1.0 & 7.3 & $31.3 \mathrm{~b}-\mathrm{d}$ \\
\hline 4. Veltyma SC $7 \mathrm{fl} \mathrm{oz}$ & 1.0 & 7.3 & $23.8 \mathrm{de}$ \\
\hline 5. Miravis Top $1.67 \mathrm{SC} 13.7 \mathrm{fl} \mathrm{oz}$ & 1.0 & 8.0 & $16.3 \mathrm{e}$ \\
\hline 6. Delaro $325 \mathrm{SC} 8 \mathrm{fl} \mathrm{oz}$ & 1.0 & 7.3 & $28.8 \mathrm{~b}-\mathrm{e}$ \\
\hline 7. Quadris Top SBX 3.76 SC $7.5 \mathrm{fl} \mathrm{oz}$ & 1.0 & 5.5 & $26.3 \mathrm{c}-\mathrm{e}$ \\
\hline 8. Domark $230 \mathrm{ME} 4 \mathrm{fl} \mathrm{oz}$ & 1.0 & 6.0 & $38.8 \mathrm{a}-\mathrm{c}$ \\
\hline 9. Topguard EQ 4.29 SC $5 \mathrm{fl} \mathrm{oz}$ & 1.0 & 8.0 & $37.5 \mathrm{a}-\mathrm{c}$ \\
\hline 10. Lucento 4.17 SC $5 \mathrm{fl} \mathrm{oz}$ & 1.0 & 5.5 & $35.0 \mathrm{a}-\mathrm{d}$ \\
\hline 11. BS-CB $10 \mathrm{~g}$ & 1.0 & 4.8 & $40.0 \mathrm{ab}$ \\
\hline 12. Untreated control 2 & 1.0 & 7.3 & $41.3 \mathrm{ab}$ \\
\hline$P(\mathrm{~F})$ & -- & 0.10 & 0.0004 \\
\hline LSD & -- & N.S. & 13.05 \\
\hline
\end{tabular}

z Foliar fungicides were applied at R3 on 28 Jul. All treatments except \#11 were applied with Induce $0.25 \% \mathrm{v} / \mathrm{v}$.

y Percent leaf area with symptoms of foliar disease.

$x$ Percent canopy defoliated as a result of disease or senescence.

Means in a column followed by the same letter(s) are not significantly different according to Fisher's Protected LSD ( $P=0.05)$.

Arcsine transformation of percentage data was made in analysis to determine statistical significance. 
Table 2. Effect of fungicide treatment on yield and grade in soybean (SOYFOLFUN120, Suffolk, VA 2020 ).

\begin{tabular}{|c|c|c|c|c|c|}
\hline Treatment, rate/A and timing ${ }^{\mathrm{z}}$ & $\begin{array}{c}\text { Yield } \\
(\mathbf{b u} / \mathbf{A})^{\mathbf{y}}\end{array}$ & $\begin{array}{c}\text { Weight/ } \\
100 \text { seed } \\
(\text { oz }) \\
\end{array}$ & $\begin{array}{c}\% \text { purple } \\
\text { seed stain }\end{array}$ & $\begin{array}{c}\% \\
\text { phomopsis } \\
\text { seed decay }^{\mathrm{x}}\end{array}$ & $\begin{array}{c}\% \text { anthrac- } \\
\text { nose }^{x}\end{array}$ \\
\hline 1. Untreated control & 62.3 & 6068 & 0.5 & $1.8 \mathrm{bc}$ & 4.0 \\
\hline 2. Revytek SC $8 \mathrm{fl} \mathrm{oz}$ & 66.2 & .5982 & 0.8 & $2.3 \mathrm{~b}$ & 6.5 \\
\hline $\begin{array}{l}\text { 3. Priaxor } 4.17 \mathrm{SC} 4 \mathrm{fl} \mathrm{oz} \\
+ \text { Tilt } 3.6 \mathrm{EC} 4 \mathrm{fl} \mathrm{oz}\end{array}$ & 63.9 & .6041 & 0.5 & $1.3 \mathrm{bc}$ & 5.8 \\
\hline 4. Veltyma SC $7 \mathrm{fl} \mathrm{oz}$ & 60.6 & .6134 & 0.3 & $0.5 \mathrm{bc}$ & 5.3 \\
\hline 5. Miravis Top $1.67 \mathrm{SC} 13.7 \mathrm{fl} \mathrm{oz}$ & 64.6 & .6140 & 0.5 & $0.8 \mathrm{bc}$ & 4.8 \\
\hline 6. Delaro $325 \mathrm{SC} 8 \mathrm{fl} \mathrm{oz}$ & 63.4 & .6143 & 0.3 & $1.5 \mathrm{bc}$ & 4.5 \\
\hline 7. Quadris Top SBX 3.76 SC $7.5 \mathrm{fl} \mathrm{oz}$ & 64.1 & .5992 & 0.0 & $4.5 \mathrm{a}$ & 6.5 \\
\hline 8. Domark $230 \mathrm{ME} 4 \mathrm{fl} \mathrm{oz}$ & 58.6 & 6159 & 0.8 & $1.0 \mathrm{bc}$ & 5.0 \\
\hline 9. Topguard EQ 4.29 SC $5 \mathrm{fl} \mathrm{oz}$ & 64.5 & .5894 & 0.3 & $0.0 \mathrm{c}$ & 3.3 \\
\hline 10. Lucento 4.17 SC $5 \mathrm{fl} \mathrm{oz}$ & 64.3 & .6052 & 0.3 & $1.5 \mathrm{bc}$ & 6.5 \\
\hline 11. BS-CB $10 \mathrm{~g}$ & 57.1 & .5949 & 0.3 & $0.5 \mathrm{bc}$ & 2.5 \\
\hline 12. Untreated control 2 & 63.2 & .6286 & 0.3 & $0.8 \mathrm{bc}$ & 3.5 \\
\hline$P(\mathrm{~F})$ & 0.30 & 0.16 & 0.92 & $\mathbf{0 . 0 2}$ & 0.43 \\
\hline LSD & N.S. & N.S. & N.S. & 2.16 & N.S. \\
\hline
\end{tabular}

z Foliar fungicides were applied at R3 on 28 Jul. All treatments except \#11 were applied with Induce $0.25 \% \mathrm{v} / \mathrm{v}$.

y Yields are weight of soybean with $13.5 \%$ moisture. One bushel equals $60 \mathrm{lb}$. Soybean was harvested on 9 Nov.

$x$ Data are percent of 100 seed with symptoms of indicated disease.

Means in a column followed by the same letter(s) are not significantly different according to Fisher's Protected LSD ( $P=0.05)$. 
TEST ID: SOYFOLFUN220

PURPOSE: To compare fungicide treatments for foliar disease control in soybean and impact on yield

LOCATION: Tidewater AREC, 6321 Holland Rd., Suffolk, VA

CROP INFORMATION:

\begin{tabular}{|l|l|}
\hline Field & 56 \\
\hline Crop history & 2019 cotton, 2018 soybean, 2017 cotton \\
\hline Planting date & 27 May \\
\hline Variety & AG48X9 \\
\hline Seeding rate & 10 seed/row ft \\
\hline Plot length/width & $30^{\prime} \times 6^{\prime}$ \\
\hline Number of rows & 4 \\
\hline Row spacing & $366^{\prime}$ \\
\hline Alleys (length between blocks) & $8^{\prime}$ \\
\hline Harvest date & 9 Nov \\
\hline
\end{tabular}

EXPERIMENTAL DESIGN: Randomized complete block with four replicates

TREATMENT APPLICATION:

\begin{tabular}{|l|l|}
\hline Equipment & Lee Spider Sprayer \\
\hline Pressure (psi) & $38 \mathrm{psi}$ \\
\hline Nozzle type & Twinjet 8002VS \\
\hline Volume (gal/A) & 19.88 \\
\hline Surfactant & NIS $0.25 \%$ v/v (except trt. 11) \\
\hline
\end{tabular}

TREATMENTS:

\begin{tabular}{|c|c|c|c|c|}
\hline Trt \# & Fungicide and formulation & Rate/A & App. timing & App. date \\
\hline 1 & Untreated & & & \\
\hline 2 & Revytek SC & $8.0 \mathrm{fl} \mathrm{oz}$ & R3 & $28 \mathrm{Jul}$ \\
\hline 3 & $\begin{array}{l}\text { Priaxor } 4.17 \mathrm{SC} \\
+ \text { Tilt } 3.6 \mathrm{EC}\end{array}$ & $\begin{array}{l}4.0 \mathrm{fl} \mathrm{oz} \\
4.0 \mathrm{fl} \mathrm{oz}\end{array}$ & R3 & $28 \mathrm{Jul}$ \\
\hline 4 & VeltyMA SC & $7.0 \mathrm{fl} \mathrm{oz}$ & R3 & $28 \mathrm{Jul}$ \\
\hline 5 & Miravis Top 1.67 SC & $13.7 \mathrm{fl} \mathrm{oz}$ & R3 & $28 \mathrm{Jul}$ \\
\hline 6 & Delaro $325 \mathrm{SC}$ & $8.0 \mathrm{fl} \mathrm{oz}$ & R3 & $28 \mathrm{Jul}$ \\
\hline 7 & Quadris Top SBX 3.76 SC & $7.5 \mathrm{fl} \mathrm{oz}$ & R3 & $28 \mathrm{Jul}$ \\
\hline 8 & Domark $230 \mathrm{ME}$ & $4.0 \mathrm{fl} \mathrm{oz}$ & $\mathrm{R} 3$ & $28 \mathrm{Jul}$ \\
\hline 9 & Topguard EQ 4.29 SC & $5.0 \mathrm{fl} \mathrm{oz}$ & $\mathrm{R} 3$ & $28 \mathrm{Jul}$ \\
\hline 10 & Lucento 4.17 SC & $5.0 \mathrm{fl} \mathrm{oz}$ & $\mathrm{R} 3$ & $28 \mathrm{Jul}$ \\
\hline 11 & BS-CB & $10.0 \mathrm{fl} \mathrm{oz}$ & R3 & $28 \mathrm{Jul}$ \\
\hline 12 & Untreated control 2 & & & \\
\hline
\end{tabular}


SOIL TYPE: Rains fine sandy loam

SOIL FERTILITY REPORT (Jan 2020):

\begin{tabular}{|l|l|l|l|l|l|l|l|l|l|}
\hline $\mathbf{p H}$ & $\mathbf{P}(\mathbf{l b} / \mathbf{A})$ & $\mathbf{K}(\mathbf{l b} / \mathbf{A})$ & $\mathbf{C a}(\mathbf{l b} / \mathbf{A})$ & $\mathbf{M g}(\mathbf{l b} / \mathbf{A})$ & $\mathbf{Z n}(\mathbf{p p m})$ & $\mathbf{M n}(\mathbf{p p m})$ & $\mathbf{C u}(\mathbf{p p m})$ & $\mathbf{F e}(\mathbf{p p m})$ & $\mathbf{B}(\mathbf{p p m})$ \\
\hline 6.5 & 91 & 197 & 1380 & 152 & 0.7 & 5.0 & 0.5 & 34.2 & 0.2 \\
\hline
\end{tabular}

MAINTENANCE CHEMICAL PROGRAMS:

\begin{tabular}{|l|l|}
\hline Fertilizer & Standard \\
\hline Herbicides & Standard \\
\hline Insecticides & Standard \\
\hline Fungicides & None except treatments \\
\hline Nematicides & None \\
\hline
\end{tabular}

MAINTENANCE CHEMICAL APPLICATIONS:

\begin{tabular}{|l|l|l|l|}
\hline Date & Type and target & Product and formulation & Rate/A \\
\hline $6 / 11 / 20$ & Herbicide & Roundup WeatherMAX & $22 \mathrm{fl} \mathrm{oz}$ \\
\hline $8 / 6 / 20$ & Herbicide & Roundup WeatherMAX & $22 \mathrm{fl} \mathrm{oz}$ \\
\hline
\end{tabular}


Table 1. Effect of fungicide treatment on foliar disease in soybean (SOYFOLFUN220, Suffolk, VA 2020).

\begin{tabular}{|c|c|c|c|}
\hline \multirow[b]{2}{*}{ Treatment and rate/ $A^{z}$} & \multicolumn{2}{|c|}{$\begin{array}{c}\text { \% foliar disease } \\
24 \text { Sep } \\
\end{array}$} & \multirow{2}{*}{$\begin{array}{c}\% \text { defoliation } \\
6 \text { Oct }\end{array}$} \\
\hline & Frog eye leaf spot & Anthracnose & \\
\hline 1. Untreated control & 1.0 & $55.0 \mathrm{a}$ & $90.0 \mathrm{ab}$ \\
\hline 2. Revytek SC $8 \mathrm{fl} \mathrm{oz}$ & 1.0 & $25.0 \mathrm{f}$ & $66.3 \mathrm{e}$ \\
\hline $\begin{array}{l}\text { 3. Priaxor } 4.17 \mathrm{SC} 4 \mathrm{fl} \mathrm{oz} \\
+ \text { Tilt } 3.6 \mathrm{EC} 4 \mathrm{fl} \mathrm{oz}\end{array}$ & 1.0 & $37.5 \mathrm{c}-\mathrm{e}$ & $80.0 \mathrm{~cd}$ \\
\hline 4. Veltyma SC $7 \mathrm{fl} \mathrm{oz}$ & 1.0 & $37.5 \mathrm{c}-\mathrm{e}$ & $73.8 \mathrm{c}-\mathrm{e}$ \\
\hline 5. Miravis Top $1.67 \mathrm{SC} 13.7 \mathrm{fl} \mathrm{oz}$ & 1.0 & $32.5 \mathrm{~d}-\mathrm{f}$ & $81.3 \mathrm{~b}-\mathrm{d}$ \\
\hline 6. Delaro $325 \mathrm{SC} 8 \mathrm{fl} \mathrm{oz}$ & 1.0 & $42.5 \mathrm{~b}-\mathrm{d}$ & $77.5 \mathrm{c}-\mathrm{e}$ \\
\hline 7. Quadris Top SBX 3.76 SC $7.5 \mathrm{fl} \mathrm{oz}$ & 1.0 & $42.5 \mathrm{~b}-\mathrm{d}$ & $71.3 \mathrm{de}$ \\
\hline 8. Domark $230 \mathrm{ME} 4 \mathrm{fl} \mathrm{oz}$ & 1.0 & $55.0 \mathrm{a}$ & $82.5 \mathrm{a}-\mathrm{c}$ \\
\hline 9. Topguard EQ 4.29 SC $5 \mathrm{fl} \mathrm{oz}$ & 1.0 & $45.0 \mathrm{a}-\mathrm{c}$ & $82.5 \mathrm{a}-\mathrm{c}$ \\
\hline 10. Lucento $4.17 \mathrm{SC} 5 \mathrm{fl} \mathrm{oz}$ & 1.0 & 30.0 ef & $77.5 \mathrm{c}-\mathrm{e}$ \\
\hline 11. BS-CB $10 \mathrm{~g}$ & 1.0 & $55.0 \mathrm{a}$ & $90.0 \mathrm{ab}$ \\
\hline 12. Untreated control 2 & 1.0 & $50.0 \mathrm{ab}$ & $91.3 \mathrm{a}$ \\
\hline$P(\mathrm{~F})$ & -- & $<0.0001$ & 0.0003 \\
\hline LSD & -- & 10.92 & 10.33 \\
\hline
\end{tabular}

z Foliar fungicides were applied at R3 on 28 Jul. All treatments except \#11 were applied with Induce $0.25 \%$ v/v.

y Percent leaf area with symptoms of foliar disease.

$x$ Percent canopy defoliated as a result of disease or senescence.

Means in a column followed by the same letter(s) are not significantly different according to Fisher's Protected LSD ( $P=0.05)$.

Arcsine transformation of percentage data was made in analysis to determine statistical significance. 
Table 2. Effect of fungicide treatment on yield and grade in soybean (SOYFOLFUN220, Suffolk, VA 2020 ).

\begin{tabular}{|c|c|c|c|c|c|}
\hline Treatment, rate/A and timing ${ }^{\mathrm{z}}$ & $\begin{array}{c}\text { Yield } \\
(\mathbf{b u} / \mathbf{A})^{y}\end{array}$ & $\begin{array}{c}\text { Weight/ } \\
100 \text { seed } \\
(\text { oz }) \\
\end{array}$ & $\begin{array}{c}\% \text { purple } \\
\text { seed stain } \\
\end{array}$ & $\begin{array}{c}\% \\
\text { phomopsis } \\
\text { seed decay }^{\mathrm{x}}\end{array}$ & $\begin{array}{l}\% \text { anthrac- } \\
\text { nose }^{x}\end{array}$ \\
\hline 1. Untreated control & 62.5 & .6616 & 2.3 & 2.3 & 3.5 \\
\hline 2. Revytek SC $8 \mathrm{fl} \mathrm{oz}$ & 63.3 & .6694 & 0.5 & 1.8 & 1.8 \\
\hline $\begin{array}{l}\text { 3. Priaxor } 4.17 \mathrm{SC} 4 \mathrm{fl} \mathrm{oz} \\
+ \text { Tilt } 3.6 \mathrm{EC} 4 \mathrm{fl} \mathrm{oz}\end{array}$ & 60.0 & .6633 & 0.5 & 3.3 & 3.0 \\
\hline 4. Veltyma SC $7 \mathrm{fl} \mathrm{oz}$ & 66.8 & .6760 & 1.5 & 2.5 & 3.0 \\
\hline 5. Miravis Top $1.67 \mathrm{SC} 13.7 \mathrm{fl} \mathrm{oz}$ & 63.3 & .6816 & 1.3 & 2.3 & 2.8 \\
\hline 6. Delaro $325 \mathrm{SC} 8 \mathrm{fl} \mathrm{oz}$ & 65.9 & 6817 & 1.5 & 0.5 & 2.5 \\
\hline $\begin{array}{l}\text { 7. Quadris Top SBX 3.76 SC } 7.5 \mathrm{fl} \\
\mathrm{oz}\end{array}$ & 66.1 & 6791 & 1.3 & 1.3 & 3.0 \\
\hline 8. Domark $230 \mathrm{ME} 4 \mathrm{fl} \mathrm{oz}$ & 63.6 & 6788 & 2.3 & 0.8 & 5.0 \\
\hline 9. Topguard EQ 4.29 SC $5 \mathrm{fl} \mathrm{oz}$ & 57.3 & .6564 & 3.0 & 1.5 & 4.5 \\
\hline 10. Lucento $4.17 \mathrm{SC} 5 \mathrm{fl} \mathrm{oz}$ & 55.5 & .6679 & 1.5 & 1.3 & 1.0 \\
\hline 11. BS-CB $10 \mathrm{~g}$ & 61.9 & .6448 & 1.8 & 2.0 & 3.3 \\
\hline 12. Untreated control 2 & 64.6 & .6507 & 2.3 & 2.8 & 4.8 \\
\hline$P(\mathrm{~F})$ & 0.27 & 0.52 & 0.60 & 0.71 & 0.41 \\
\hline LSD & N.S. & N.S. & N.S. & N.S. & N.S. \\
\hline
\end{tabular}

$\mathrm{z}$ Foliar fungicides were applied at R3 $28 \mathrm{Jul}$. All treatments except \#11 were applied with Induce $0.25 \% \mathrm{v} / \mathrm{v}$.

y Yields are weight of soybean with $13.5 \%$ moisture. One bushel equals $60 \mathrm{lb}$. Soybean was harvested on 9 Nov.

$x$ Data are percent of 100 seed with symptoms of indicated disease. 


\section{CLIMATOLOGICAL SUMMARY OF THE 2020 GROWING SEASON AT THE}

TIDEWATER AGRICULTURAL RESEARCH \& EXTENSION CENTER, SUFFOLK, VA.

\begin{tabular}{|c|c|c|c|c|c|c|c|c|c|c|c|c|}
\hline \multirow{2}{*}{$\begin{array}{l}\text { Day of } \\
\text { month }\end{array}$} & \multicolumn{2}{|c|}{ NOV } & \multicolumn{2}{|c|}{ DEC } & \multicolumn{2}{|c|}{ JAN } & \multicolumn{2}{|c|}{ FEB } & \multicolumn{2}{|c|}{ MAR } & \multicolumn{2}{|c|}{ APR } \\
\hline & Max. & Min. & Max. & Min. & Max. & Min. & Max. & Min. & Max. & Min. & Max. & Min. \\
\hline 1 & 74 & 40 & 64 & 43 & 56 & 31 & 47 & 42 & 53 & 21 & 55 & 41 \\
\hline 2 & 63 & 36 & 51 & 41 & 61 & 32 & 58 & 35 & 70 & 36 & 64 & 41 \\
\hline 3 & 61 & 36 & 52 & 36 & 58 & 45 & 71 & 44 & 70 & 46 & 72 & 42 \\
\hline 4 & 67 & 33 & 54 & 32 & 63 & 47 & 70 & 58 & 63 & 43 & 60 & 40 \\
\hline 5 & 71 & 50 & 54 & 30 & 51 & 31 & 69 & 45 & 53 & 44 & 71 & 37 \\
\hline 6 & 61 & 38 & 56 & 26 & 59 & 30 & 74 & 47 & 55 & 41 & 77 & 50 \\
\hline 7 & 72 & 36 & 49 & 26 & 57 & 28 & 64 & 37 & 54 & 29 & 80 & 50 \\
\hline 8 & 58 & 33 & 58 & 23 & 55 & 27 & 49 & 27 & 61 & 26 & 83 & 61 \\
\hline 9 & 55 & 27 & 65 & 48 & 48 & 25 & 55 & 31 & 71 & 40 & 80 & 53 \\
\hline 10 & 64 & 29 & 75 & 53 & 69 & 32 & 64 & 33 & 72 & 58 & 58 & 38 \\
\hline 11 & 69 & 39 & 53 & 31 & 74 & 52 & 72 & 60 & 70 & 49 & 64 & 29 \\
\hline 12 & 56 & 30 & 45 & 29 & 72 & 56 & 58 & 48 & 73 & 49 & 76 & 44 \\
\hline 13 & 41 & 23 & 55 & 30 & 69 & 56 & 65 & 48 & 76 & 53 & 81 & 59 \\
\hline 14 & 48 & 21 & 59 & 46 & 67 & 55 & 54 & 33 & 65 & 40 & 65 & 51 \\
\hline 15 & 49 & 41 & 58 & 38 & 62 & 49 & 43 & 23 & 53 & 46 & 56 & 37 \\
\hline 16 & 48 & 43 & 69 & 37 & 64 & 41 & 52 & 28 & 56 & 44 & 65 & 33 \\
\hline 17 & 48 & 42 & 66 & 41 & 44 & 26 & 57 & 34 & 67 & 45 & 71 & 36 \\
\hline 18 & 46 & 43 & 47 & 30 & 48 & 31 & 62 & 34 & 63 & 48 & 66 & 42 \\
\hline 19 & 48 & 43 & 40 & 24 & 57 & 31 & 57 & 31 & 82 & 51 & 70 & 36 \\
\hline 20 & 58 & 34 & 49 & 22 & 38 & 24 & 44 & 32 & 86 & 68 & 62 & 48 \\
\hline 21 & 56 & 31 & 46 & 29 & 39 & 21 & 37 & 19 & 76 & 47 & 75 & 48 \\
\hline 22 & 65 & 46 & 51 & 26 & 45 & 20 & 48 & 15 & 57 & 46 & 67 & 35 \\
\hline 23 & 56 & 36 & 55 & 38 & 49 & 26 & 62 & 27 & 51 & 46 & 65 & 46 \\
\hline 24 & 61 & 38 & 58 & 33 & 64 & 35 & 54 & 32 & 61 & 46 & 79 & 55 \\
\hline 25 & 61 & 30 & 61 & 34 & 62 & 39 & 64 & 48 & 56 & 47 & 65 & 53 \\
\hline 26 & 68 & 33 & 64 & 32 & 53 & 32 & 63 & 52 & 62 & 41 & 74 & 54 \\
\hline 27 & 61 & 47 & 64 & 39 & 56 & 29 & 57 & 32 & 74 & 51 & 62 & 44 \\
\hline 28 & 59 & 39 & 70 & 45 & 52 & 29 & 53 & 26 & 85 & 55 & 71 & 40 \\
\hline 29 & 54 & 31 & 65 & 44 & 49 & 25 & 48 & 28 & 88 & 62 & 80 & 53 \\
\hline 30 & 48 & 39 & 77 & 55 & 50 & 29 & & & 77 & 59 & 72 & 59 \\
\hline 31 & & & & & 54 & 29 & & & 61 & 45 & & \\
\hline Average & 58 & 36 & 58 & 35 & 56 & 34 & 58 & 36 & 67 & 46 & 70 & 45 \\
\hline Normal & 64 & 39 & 56 & 33 & 51 & 29 & 55 & 31 & 61 & 37 & 72 & 47 \\
\hline $\begin{array}{c}\begin{array}{c}\text { Deviation } \\
\text { from } \\
\text { normal }\end{array} \\
\end{array}$ & -6 & -3 & +2 & +2 & +5 & +5 & +3 & +5 & +6 & +9 & -3 & -2 \\
\hline
\end{tabular}

${ }^{z}$ Data collected with Watchdog Weather station Series 2450 (Spectrum Technologies, Aurora, IL) located at the Tidewater Research Farm, Hare Rd., Suffolk, VA. Normal monthly temperature means determined using 15-yr data (2005-2019). 


\begin{tabular}{|c|c|c|c|c|c|c|c|c|c|c|c|c|}
\hline \multirow{2}{*}{$\begin{array}{l}\text { Day of } \\
\text { month }\end{array}$} & \multicolumn{2}{|c|}{ MAY } & \multicolumn{2}{|c|}{ JUN } & \multicolumn{2}{|c|}{ JUL } & \multicolumn{2}{|c|}{ AUG } & \multicolumn{2}{|c|}{ SEP } & \multicolumn{2}{|c|}{ OCT } \\
\hline & Max. & Min. & Max. & Min. & Max. & Min. & Max. & Min. & Max. & Min. & Max. & Min. \\
\hline 1 & 64 & 53 & 75 & 54 & 91 & 70 & 92 & 75 & 86 & 71 & 82 & 52 \\
\hline 2 & 72 & 48 & 84 & 52 & 90 & 69 & 93 & 74 & 92 & 74 & 72 & 50 \\
\hline 3 & 86 & 54 & 92 & 69 & 96 & 70 & 88 & 72 & 94 & 77 & 70 & 46 \\
\hline 4 & 80 & 57 & 93 & 73 & 91 & 72 & 89 & 71 & 93 & 73 & 69 & 47 \\
\hline 5 & 62 & 48 & 88 & 71 & 91 & 68 & 92 & 73 & 82 & 60 & 74 & 47 \\
\hline 6 & 68 & 51 & 91 & 74 & 93 & 69 & 91 & 72 & 83 & 57 & 77 & 45 \\
\hline 7 & 67 & 46 & 85 & 65 & 92 & 71 & 88 & 73 & 84 & 55 & 82 & 54 \\
\hline 8 & 71 & 46 & 89 & 62 & 86 & 72 & 89 & 72 & 86 & 65 & 80 & 53 \\
\hline 9 & 60 & 37 & 91 & 65 & 87 & 75 & 89 & 72 & 80 & 74 & 75 & 50 \\
\hline 10 & 69 & 33 & 88 & 71 & 92 & 74 & 92 & 71 & 85 & 75 & 67 & 61 \\
\hline 11 & 69 & 49 & 83 & 73 & 92 & 73 & 91 & 71 & 87 & 75 & 72 & 66 \\
\hline 12 & 65 & 35 & 81 & 66 & 94 & 69 & 92 & 72 & 80 & 64 & 81 & 68 \\
\hline 13 & 72 & 42 & 79 & 61 & 92 & 71 & 89 & 73 & 84 & 64 & 75 & 56 \\
\hline 14 & 79 & 49 & 78 & 56 & 94 & 68 & 87 & 75 & 83 & 62 & 76 & 47 \\
\hline 15 & 84 & 61 & 65 & 61 & 92 & 69 & 86 & 73 & 73 & 57 & 80 & 46 \\
\hline 16 & 88 & 63 & 66 & 61 & 88 & 67 & 77 & 70 & 78 & 53 & 70 & 53 \\
\hline 17 & 80 & 63 & 79 & 66 & 95 & 71 & 85 & 69 & 72 & 64 & 65 & 41 \\
\hline 18 & 69 & 63 & 83 & 64 & 98 & 73 & 92 & 68 & 74 & 62 & 74 & 40 \\
\hline 19 & 63 & 54 & 84 & 64 & 99 & 74 & 88 & 67 & 67 & 52 & 78 & 48 \\
\hline 20 & 60 & 54 & 82 & 67 & 101 & 79 & 86 & 69 & 71 & 52 & 83 & 62 \\
\hline 21 & 70 & 57 & 88 & 67 & 100 & 76 & 87 & 68 & 66 & 49 & 85 & 61 \\
\hline 22 & 83 & 68 & 92 & 70 & 100 & 74 & 88 & 73 & 71 & 47 & 81 & 55 \\
\hline 23 & 84 & 67 & 90 & 70 & 98 & 74 & 89 & 74 & 79 & 48 & 78 & 56 \\
\hline 24 & 70 & 61 & 89 & 67 & 91 & 73 & 84 & 73 & 69 & 55 & 84 & 56 \\
\hline 25 & 75 & 60 & 83 & 67 & 96 & 72 & 91 & 73 & 68 & 61 & 68 & 57 \\
\hline 26 & 72 & 63 & 88 & 65 & 99 & 71 & 90 & 69 & 78 & 66 & 63 & 57 \\
\hline 27 & 82 & 66 & 91 & 67 & 100 & 75 & 93 & 69 & 77 & 64 & 65 & 59 \\
\hline 28 & 83 & 73 & 90 & 71 & 102 & 75 & 94 & 74 & 84 & 62 & 76 & 58 \\
\hline 29 & 85 & 74 & 92 & 71 & 90 & 74 & 86 & 77 & 79 & 63 & 82 & 64 \\
\hline 30 & 87 & 70 & 93 & 69 & 95 & 73 & 91 & 68 & 72 & 54 & 76 & 45 \\
\hline 31 & 75 & 56 & & & 97 & 77 & 86 & 64 & & & 61 & 40 \\
\hline Average & 74 & 56 & 85 & 66 & 94 & 72 & 89 & 71 & 79 & 62 & 75 & 53 \\
\hline Normal & 80 & 57 & 88 & 65 & 91 & 68 & 89 & 67 & 84 & 62 & 74 & 50 \\
\hline $\begin{array}{c}\begin{array}{c}\text { Deviation } \\
\text { from } \\
\text { normal }\end{array} \\
\end{array}$ & -6 & -1 & -3 & +1 & +4 & +4 & 0 & +4 & -5 & $\mathbf{0}$ & +1 & +3 \\
\hline
\end{tabular}

z Data collected with Watchdog Weather station Series 2450 (Spectrum Technologies, Aurora, IL) located at the Tidewater Research Farm, Hare Rd., Suffolk, VA. Normal monthly temperature means determined using 15-yr data (2005-2019). 


\begin{tabular}{|c|c|c|c|c|c|c|}
\hline $\begin{array}{l}\begin{array}{l}\text { Day of } \\
\text { month }\end{array} \\
\end{array}$ & NOV & DEC & JAN & FEB & MAR & APR \\
\hline 1 & 0.36 & 0.35 & 0 & 0.17 & 0 & 0 \\
\hline 2 & 0 & 0.12 & 0 & 0.01 & 0 & 0.01 \\
\hline 3 & 0 & 0 & 0.52 & 0 & 0.02 & 0 \\
\hline 4 & 0 & 0 & 0.57 & 0 & 0 & 0 \\
\hline 5 & 0.11 & 0 & 0.19 & 0.04 & 0 & 0 \\
\hline 6 & 0 & 0 & 0 & 0.46 & 0.26 & 0 \\
\hline 7 & 0.01 & 0 & 0.17 & 0.13 & 0 & 0 \\
\hline 8 & 0.08 & 0.02 & 0 & 0.49 & 0 & 0 \\
\hline 9 & 0 & 0.62 & 0 & 0 & 0 & 0 \\
\hline 10 & 0 & 0 & 0 & 0 & 0 & 0 \\
\hline 11 & 0 & 0.35 & 0 & 0.16 & 0 & 0 \\
\hline 12 & 0.53 & 0 & 0.01 & 0 & 0 & 0 \\
\hline 13 & 0 & 0.75 & 0.25 & 0.2 & 0.04 & 0.34 \\
\hline 14 & 0 & 0.25 & 1.1 & 0 & 0 & 0 \\
\hline 15 & 0.66 & 0 & 0 & 0 & 0 & 0.27 \\
\hline 16 & 0 & 0 & 0 & 0 & 0 & 0 \\
\hline 17 & 0 & 0.24 & 0 & 0 & 0 & 0 \\
\hline 18 & 0.08 & 0 & 0.1 & 0.01 & 0 & 0.64 \\
\hline 19 & 0 & 0 & 0.02 & 0.23 & 0.08 & 0.03 \\
\hline 20 & 0 & 0 & 0 & 0.04 & 0 & 0.75 \\
\hline 21 & 0 & 0 & 0 & 0.42 & 0.05 & 0 \\
\hline 22 & 0 & 0 & 0 & 0 & 0 & 0 \\
\hline 23 & 0.39 & 0 & 0 & 0.01 & 0.24 & 0 \\
\hline 24 & 0.19 & 0 & 0 & 0.01 & 0 & 0.8 \\
\hline 25 & 0 & 0 & 0.67 & 0.04 & 0 & 0 \\
\hline 26 & 0 & 0 & 0 & 0.03 & 0.01 & 0.09 \\
\hline 27 & 0.15 & 0 & 0 & 0.07 & 0.02 & 0.02 \\
\hline 28 & 0 & 0 & 0 & 0 & 0.01 & 0 \\
\hline 29 & 0 & 0.02 & 0 & 0 & 0.01 & 0 \\
\hline 30 & 0 & 0.04 & 0 & & 0 & 1.63 \\
\hline 31 & & & 0.34 & & 0 & \\
\hline Total & 2.56 & 2.76 & 3.94 & 2.52 & 0.74 & 4.58 \\
\hline Normal & 3.36 & 3.47 & 3.25 & 3.29 & 3.33 & 3.19 \\
\hline $\begin{array}{c}\text { Deviation } \\
\text { from } \\
\text { normal } \\
\end{array}$ & -0.80 & -0.71 & +0.69 & -0.77 & -2.59 & 1.39 \\
\hline
\end{tabular}

${ }^{z}$ Data collected with Watchdog Weather station Series 2450 (Spectrum Technologies, Aurora, IL) located at the Tidewater Research Farm, Hare Rd., Suffolk, VA. Normal monthly rainfall total determined using 15-yr data (2005-2019). 


\begin{tabular}{|c|c|c|c|c|c|c|}
\hline $\begin{array}{l}\begin{array}{l}\text { Day of } \\
\text { month }\end{array} \\
\end{array}$ & MAY & JUN & JUL & AUG & SEP & OCT \\
\hline 1 & 0.1 & 0 & 0.05 & 0.18 & 0.97 & 0 \\
\hline 2 & 0 & 0 & 0 & 0 & 0 & 0 \\
\hline 3 & 0 & 0 & 0 & 1.15 & 0 & 0 \\
\hline 4 & 0 & 0 & 0 & 1.0 & 0 & 0 \\
\hline 5 & 0 & 0.06 & 0 & 0.2 & 0 & 0 \\
\hline 6 & 0.47 & 0.01 & 0 & 1.53 & 0 & 0 \\
\hline 7 & 0 & 1.1 & 0 & 0.01 & 0 & 0 \\
\hline 8 & 0.02 & 0 & 1.07 & 1.23 & 0 & 0 \\
\hline 9 & 0.02 & 0 & 0.02 & 0 & 2.28 & 0 \\
\hline 10 & 0 & 0 & 0 & 0 & 0.97 & 0.22 \\
\hline 11 & 0 & 0.01 & 0 & 0 & 0.13 & 0.27 \\
\hline 12 & 0 & 0.49 & 0 & 0 & 0 & 0.2 \\
\hline 13 & 0 & 0 & 0 & 0.97 & 0 & 0 \\
\hline 14 & 0 & 0 & 0.24 & 0.18 & 0.01 & 0 \\
\hline 15 & 0 & 0.4 & 0 & 0.94 & 0 & 0 \\
\hline 16 & 0 & 0.96 & 0 & 0.06 & 0 & 0.1 \\
\hline 17 & 0 & 0.16 & 0 & 0 & 1.29 & 0 \\
\hline 18 & 0.56 & 0 & 0 & 0 & 2.44 & 0 \\
\hline 19 & 0.25 & 0 & 0 & 0.03 & 0 & 0 \\
\hline 20 & 0.02 & 0.01 & 0 & 0.01 & 0 & 0 \\
\hline 21 & 0.21 & 0 & 0 & 0.33 & 0 & 0 \\
\hline 22 & 0.43 & 0.62 & 0 & 0.57 & 0 & 0 \\
\hline 23 & 0.03 & 0 & 0.09 & 0.01 & 0 & 0 \\
\hline 24 & 0 & 0 & 0 & 0 & 0 & 0 \\
\hline 25 & 0 & 0.01 & 0 & 0 & 0.19 & 0.25 \\
\hline 26 & 0 & 0 & 0 & 0 & 0.22 & 0 \\
\hline 27 & 0.2 & 0 & 0 & 0 & 0 & 0 \\
\hline 28 & 0.52 & 0 & 0.19 & 0 & 0 & 0 \\
\hline 29 & 0.26 & 0 & 0 & 0.05 & 1.52 & 0.03 \\
\hline 30 & 0.41 & 0 & 0 & 0 & 0.18 & 0.25 \\
\hline 31 & 0 & & 0.43 & 0.05 & & 0 \\
\hline Total & 3.50 & $\mathbf{3 . 8 3}$ & 2.09 & 8.50 & 10.20 & 1.32 \\
\hline Normal & 4.07 & 4.30 & 4.71 & 5.36 & 5.67 & 4.46 \\
\hline $\begin{array}{c}\text { Deviation } \\
\text { from } \\
\text { normal } \\
\end{array}$ & +0.57 & -0.47 & -2.62 & +3.14 & +4.53 & -3.14 \\
\hline
\end{tabular}

z Data collected with Watchdog Weather station Series 2450 (Spectrum Technologies, Aurora, IL) located at the Tidewater Research Farm, Hare Rd., Suffolk, VA. Normal monthly rainfall total determined using 15-yr data (2005-2019). 